
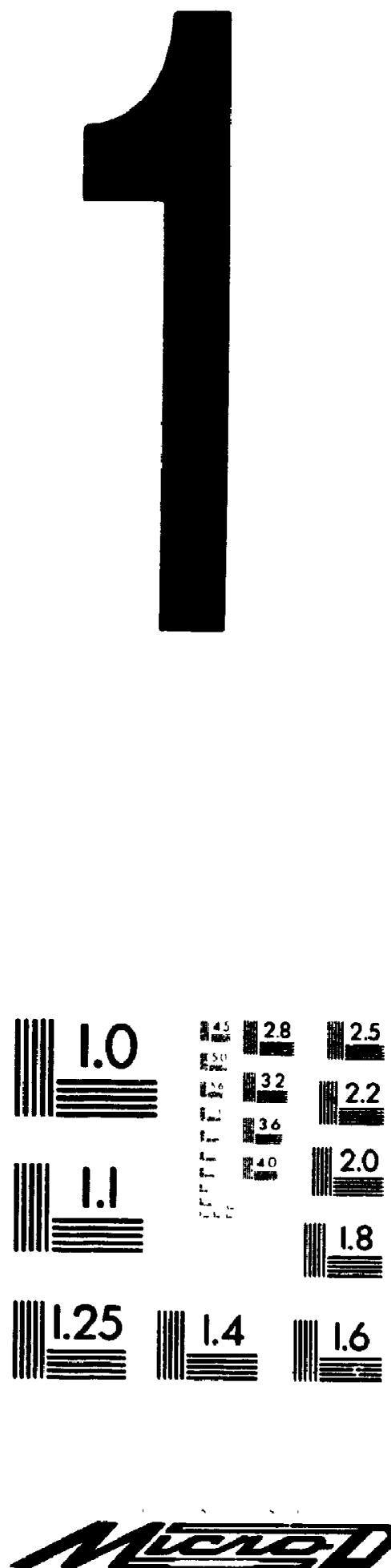
Otawa, Canada

KTA ON4

\section{Service des theses canadiennes}

\section{NOTICE}

The quality of this microform is heavily dependent upon the quality of the original thesis submitted for microfilming. Every effort has been made to ensure the highest quality of reproduction possible.

If pages are missing. contact the university which granted the degree.

Some pages may have indistinct print especially if the original pages were typerd with a poor typewriter ribbon or if the university sent us an inferior photocopy.

Reproduction in full or in part of this microlorm is governed by the Canadian Copyright ACt, R.S.C. 1970, C. C-30, and subsequent amendments.

\section{AVIS}

La qualité de celte microforme dépend grandement de la qualité de la thèse soumise au microfilmage. Nous avons tout tait pour assurer une qualité supérieure de reproduction.

S'il manque des pages, veuillez communiquer avec l'université qui a conféré le grade.

La qualité d'impression de certaines pages peut laisser à désirer. surtout sı les pages originales ont élé dactylographiées à l'aide d'un ruban usé ou si runiversité nous a tait parvenir une photocopie de qualité intérieure.

La reproduction, meme partielle, de cette microlorme est soumise a la Lol canadienne sur le droit d'auteur, SRC 1970, c. C-30, et ses amendements subséquents. 


\title{
Investigation of the Gate Capacitance Characteristics of Short Channel MOSFETs
}

\author{
by \\ Christos P. Karabatsos B.Sc., (Physics)
}

A thesis submitted to the Faculty of Graduate Studies and Research in partial fulfillment of the requirements for the degree of

Master of Engineering

Ottawa-Carleton Institute for Electrical Engineering

Department of Electronics

Faculty of Engineering

Carleton University

(C) copyright

1989, C. P. Karabatsos 
The author has granted an irrevocable nonexclusive licence allowing the National Library of Canada to reproduce, loan, distribute or sell copies of his/her thesis by any means and in any form or format, making this thesis available to interested persons.

The author retains ownership of the copyright in his/her thesis. Neither the thesis nor substantial extracts from it may be printed or otherwise reproduced without his/her permission.
L'auteur a accordé une licence irrévocable et non exclusive permettant à la Bibliothéque nationale du Canada de reproduire, preter. distribuer ou vendre des copies de sa these de quelque maniere et sous quelque forme que ce soit pour mettre des exemplaires de cette thèse à la disposition des personnes intéresseees.

L'auteur conserve la propriété du cirnit d'auteur qui protège sa thèse. Ni la thèse ni des extraits substantiels de celle-ci ne doivent etre imprimés ou autrement reproduits sans son autorisation. 
The undersigned recommend to the Faculty of Graduate Studies and Research the acceptance of the thesis:

Investigation of the Gate Capacitance Characteristics of Short Channel MOSFETs

submitted by Christos P. Karabatsos in partial fulfillment of the requirements for the degree of Master of Engineering.

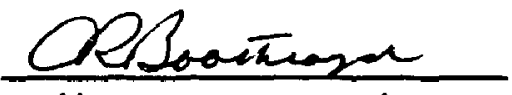

Professor A. R. Boothroyd Thesis Supervisor

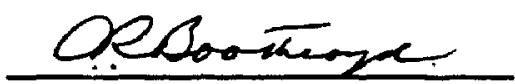

Professor A. R. Boothroyd Acting Chairman Department of Electronics

Carleton University

Date: b octoten 1; 59 


\section{Abstract}

VLSI continues to impose demands to better understand and model both the steady state and dynamic behavior of s.. channel MOSFETs, the latter being the object of the present study. This work presents a study of the gate capacitance characteristics of short channel devices using MIN. IMOS, a 2D numerical simulation package for the planar MOS transistor.

Although it was found to be possible to study the capacitance characteristics of short channel MOSFETs with MINIMOS, the presence of numerical scatter, a product of the automatic generation of the grid in MINIMOS, limited the amount of useful information provided by the simulator. The scatter of data vas significantly reduced by performing consecutive simulations using a procedure which ensures a fixed grid set at the highrst bias conditions. It was concluded that MINIMOS could be an effective tool in the study of short channel capacitances, if the grid were carefully designed to provide the necessary pattern and resolution.

An analytical approach to modelling short channel capacitances was also investigated, since such a model would be of practical use in circuit simulation applications. The model estimates the surface potential in the 2D source and drain space charge regions by solving Poison's equation using cylindrical coordinates in the $1 \mathrm{D}$ \& dial direction. It was demonstrated that it is possible to predict $C_{G D}$ characteristics in the subthreshold and saturation regions using this model. The ability to predict accurate short channel capacitance characteristics using a practical analytical model will depend largely on the degree of sophistication needed in order to adequately represent the true 2D nature of the source and drain space charge regions. 


\section{Acknowledgement}

As I believe that the most meaningful and important lessons are taught by setting the example, as opposed to any other form of direct communication, I consider myself extremely fortunate for having the privilege of supervision by Professor Albert Roy Boothroyd on this thesis. His kindness, patience and lucid understanding of the scientific method, an well as the humanities, has provided me with valuable knowledge and rich experience that far exceeds the scope of the present thesis. For all that, I am grateful.

I am also grateful to my wife Mirella Aiello, whose selfless love, understanding and kind support, has heiped me realize my goal.

Many thanks are also due to Ardavan Tajbakhsh and Colin McFadyen for their invaluable and assistance. 
To my parents Panugiotis and Chryssanthi Karabatsos, with love 


\section{Table of Contents}

\section{Chapter 1 Introduction}

\section{Chapter 2 Transient Analysis of the MOSFET}

2.1 Dynamic Analysis of the MOS Transistor . . . . . . . . . . . 3

2.2 Dynamic Modelling Methods . . . . . . . . . . . . . . . . . . 8

2.2.1 Numerical Methods of Modelling Capacitances . . . . . . . . . . 9

i. Capacitance-Based Method . . . . . . . . . . . . 9

ii. Charge-Based Method . . . . . . . . . . . . . . . . 11

2.2.2 Capacitance Matrix . . . . . . . . . . . . . . . . . 13

i. Full Capacitance Matrix . . . . . . . . . . . . . 13

ii. Simplified Gate Capacitance Model: Meyer's Model . . . . . 15

2.3 Evaluation of Terminal Currents in Quasi-Static Operation . . 19

2.3.1 Methods for Partitioning the Mobile Channel Charge $Q_{M} \quad$. . . . 24

2.4 Short Channel MOS Transistor Capacitances . . . . . . . . . 27

Chapter 3 Extraction of Gate Capacitances from MINIMOS Simulation

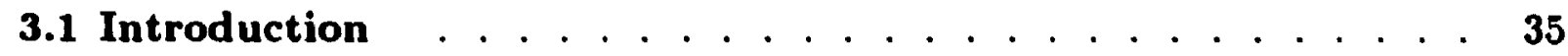

3.2 Feasibility of MINIMOS for Investigating Capacitance

Characteristics . . . . . . . . . . . . . . . . . 37

3.2.1 Surface Potential Distribution $\psi_{s}(y)$. . . . . . . . . . . . . . 37

3.2.2 Calculating the Gate Charge $Q_{G}$. . . . . . . . . . . . . . . . 40

3.2.3 Interpretation of Results with $\mathcal{E}_{s i}$. . . . . . . . . . . . . . . . 49

3.3 Extracting Gate Capacitances . . . . . . . . . . . . . . . 51 
3.3.1 Introduction . . . . . . . . . . . . . . . . . . . . 51

3.3.2 Study of First Simulation Procedure . . . . . . . . . . . . . . 52

3.3.3 Study of Improved Simulation Procedure . . . . . . . . . . . . 56

\section{Chapter 4 Discussion of Results of Capacitance Studies}

4.1 Capacitance Data from MINIMOS Simulation . . . . . . . . . 63

4.1.1 Accuracy of Results . . . . . . . . . . . . . . . . . . . . . . 63

4.1.2 Smoothing of Data . . . . . . . . . . . . . . . . . . . 70

4.2 Gate Self-Capacitance $C_{G G}$. . . . . . . . . . . . . . . . . . . 72

4.3 Capacitance Component $C_{G D} \ldots \ldots \ldots$

4.4 Capacitance Component $C_{G S} \ldots \ldots . . \ldots 77$

4.5 Capacitance Component $C_{G B} \ldots \ldots \ldots$

4.6 Concluding Remarks . . . . . . . . . . . . . . . . . . . . . . 79

\section{Chapter 5 Analytical Modelling of Capacitance}

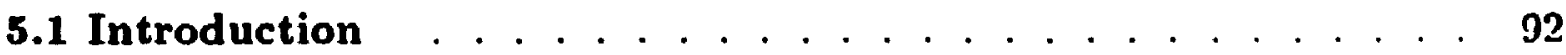

5.2 A nalytical Modelling of the 2D Surface Potential . . . . . . . 02

5.2.1 Approximating $\psi_{s}(y)$ in the Source and Drain Space Charge Regions:

The Cylindrical Step Junction Model . . . . . . . . . . . . . . 93

5.2.2 Quadratic Approximation of $\psi_{s}(y)$ for the Reduced 1D Channel Region 96

5.2.3 Division of the Channel and the Determination of Boundaries . . 99

5.3 Gate Capacitance Component $C_{G D}$. . . . . . . . . . . . . . 101

5.3.1 Evaluation of $C_{G D}$ Using Analytical Approach . . . . . . . . . 101

5.3.2 Comparison of Analytical Results With Those From MINIMOS . 107

5.4 Concluding Remarks . . . . . . . . . . . . . . . . . . . . 110 
viii

Chapter 6 Conclusions

6.1 Summary . . . . . . . . . . . . . . . . . . . . . . . . . . . 113

6.2 Suggestions for Further Studies . . . . . . . . . . . . . . . 115

References . . . . . . . . . . . . . . . . . . . . . . . . 116 


\section{List of Figures}

Figure 2.1: Structure of MOSFET showing the intrinsic and extrinsic regions ....4

Figure 2.2 : A non-linear two-terminal capacitor with corresponding quantities defined for simulation in a circuit simulation environment $\ldots \ldots \ldots 6$

Figure 2.3: Gate-drain capacitances $C_{G D}$ and $C_{D G}$ in MOSFET $\ldots \ldots \ldots \ldots .15$

Figure 2.4(a) : Circuit representation of Meyer's Model $\ldots \ldots \ldots \ldots \ldots \ldots \ldots \ldots$

Figure 2.4(b) : Meyer's simplified capacitance matrix $\ldots \ldots \ldots \ldots \ldots \ldots \ldots \ldots$

Figure 2.5 : $C_{G D}, C_{G S}, C_{G B}$ from Meyer's model $\ldots \ldots \ldots \ldots \ldots \ldots \ldots \ldots \ldots$

Figure 2.6 : Gate voltage waveform (a), causing a change in mobile charge $Q_{M}$

(b), being reflected in the total source and drain current changes (c) .21

Figure 2.7 : Capacitances obtained from measurement; a) $C_{G D}, C_{G S}, C_{G B}$

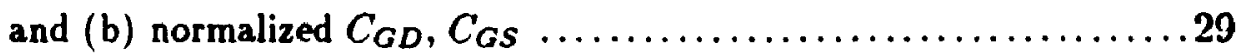

Figure 2.8 : Normalized gate capacitances obtained from 2D simulation, with velocity saturation (solid), constant mobility (dashed) and measure. ment (dotted); $V_{B S}=-2.0 \mathrm{~V}, V_{D S}=2.0 \mathrm{~V}$; a) $L_{e} \int f=0.8 \mu \mathrm{m}$, b) $L_{e f f}=7.8 \mu \mathrm{m} . C_{G D(e x)}$ and $C_{G S(e x)}$ are extrinsic capacitance components due to outer fringing coupling effects.

Figure 2.9 : $\Delta \psi_{\text {, }}$-distribution with and without velocity saturation; linear region: $V_{D S}$ changes from $2.00 \mathrm{~V}$ to $2.01 \mathrm{~V} . V_{G S}=4.0 \mathrm{~V}, V_{B S}=$ $-2.0 \mathrm{~V} ; L_{e f f}=0.8 \mu \mathrm{m}$.

Figure 3.1: $\quad \psi_{\mathbf{s}}(y)$ from MINIMOS and long channel theory for saturation $\left(V_{G S}=3.35 \mathrm{~V}\right)$ and triode $\left(V_{G S}=3.75 \mathrm{~V}\right)$ region of operation: $V_{D S}=2.0 \mathrm{~V}, V_{S B}=0 \mathrm{~V} ; L_{e f f}=49.3 \mu \mathrm{m} \ldots \ldots \ldots \ldots \ldots \ldots . \ldots \ldots$

Figure 3.2: $\quad \psi_{\text {s }}(y)$ from MINIMOS and long channel theory for saturation $\left(V_{G i S}=3.0 V\right)$ and triode $\left(V_{G S}=3.5 V\right)$ region of operation; 
$V_{D S}=2.0 \mathrm{~V}, V_{S B}=0 \mathrm{~V} ; L_{e f f}=2.3 \mu \mathrm{m}$

Figure 3.3 : Distributions of $\mathcal{E}_{o x}(y)$ (solid), $\mathcal{E}_{\Delta i}(y)$ (dashed) and $\mathcal{E}_{o i}(y) \mathcal{E}_{o s}$

dotted, for $V_{D S}=2.0 \mathrm{~V} ; L_{e / f}=49.3 \mu \mathrm{m}$

Figure 3.4(a): $Q_{G}\left(\mathcal{E}_{o x}\right) \sim V_{G S} ;$ gate charge $Q_{G}\left(\mathcal{E}_{o x}\right)$ is evaluated by integrating the transverse electric field inside the oxide $\mathcal{E}_{o x}$ for incremental gate voltages $\Delta V_{G S}=0.1 \mathrm{~V} ; V_{D S}=2.0 \mathrm{~V}, L_{e f f}=2.3 \mu \mathrm{m}$

Figure 3.4(b): $C_{G G}\left(\varepsilon_{o x}\right) \sim V_{G S}$ characteristics evaluated using the first simulation procedure showing all the discontinuities; $C_{G G}\left(\mathcal{E}_{o x}\right)$ is evaluated by taking $\Delta Q_{G}\left(\mathcal{E}_{o x}\right) / \Delta V_{G S} ; V_{D S}=2.0 \mathrm{~V}, L_{\text {eff }}=2.3 \mu \mathrm{m}$

Figure 3.4(c) : $C_{G G}\left(\mathcal{E}_{o x}\right) \sim V_{G S}$ characteristics evaluated using the first simulation procedure after removing the major discontinuities

Figure 3.5 : $\quad C_{G G}\left(\psi_{S}\right) \sim V_{G S}$ characteristics evaluated using the first simulation procedure after all major discontinuities have been removed; $C_{G G}\left(\psi_{\mathrm{a}}\right)$ is evaluated by taking $\Delta Q_{G}\left(\psi_{a}\right) / \Delta V_{G S} ; V_{D S}=2.0 \mathrm{~V}$, $L_{\text {eff }}=2.3 \mu \mathrm{m}$.

Figure 3.6 : Illustration showing the method by which the range of $V_{G S}$ is segmented into the different sets iof hopping intervals indicated by $D G$, in order to include all the necessary points of simulation

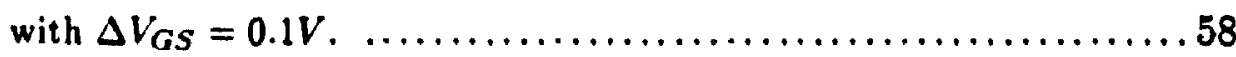

Figure 3.7 : (a) 3D view of surface of $Q_{G}$ points, of which the curve of all $Q_{G 1}$ values is shown for $V_{D S 1}$; (b) $2 D$ projection of the surface of $Q_{G}$ points on the $Q_{G} \sim V_{D S}$ axis, cut at $V_{G S 1}$, with the expanded area showing the two values of $Q_{G 1}$ and $Q_{G 2}$ at $V_{D S 1}$ and $I_{D S 2}$, respectively; (c) 2D progection of both $Q_{G}$ curves on $Q_{G}$

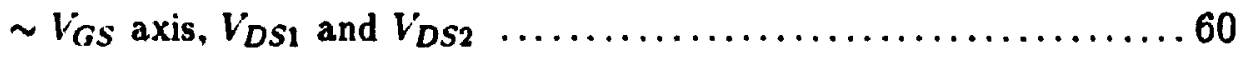

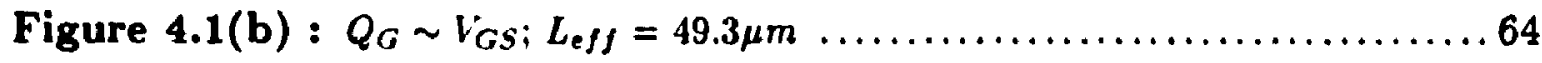

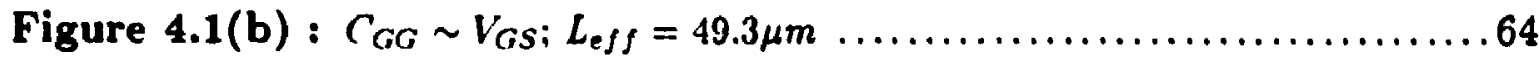

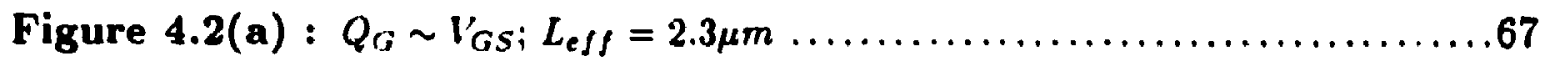

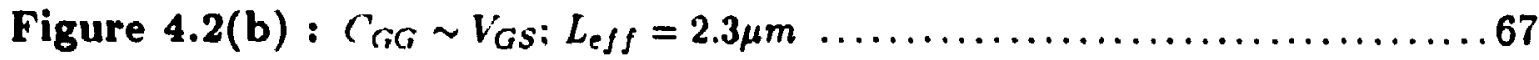

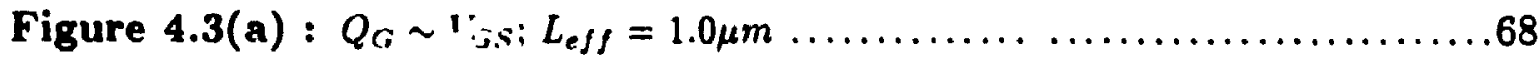




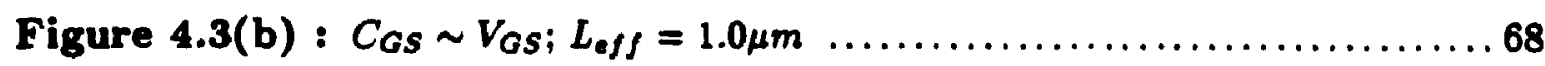

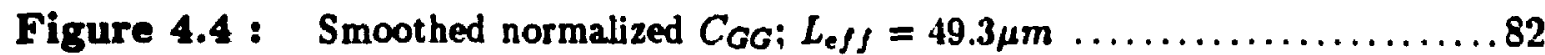

Figure 4.5 : Smoothed normalized $C_{G G} ; L_{e f f}=2.3 \mu m \ldots \ldots \ldots \ldots \ldots . \ldots . \ldots . \ldots 2$

Figure 4.6 : Smoothed normalized $C_{G G} ; L_{\text {eff }}=1.0 \mu m \ldots \ldots \ldots \ldots \ldots \ldots . \ldots . \ldots . \ldots$

Figure 4.7 : Smoothed normalized $C_{G D} ; L_{e f f}=49.3 \mu m \ldots \ldots \ldots \ldots \ldots \ldots$

Figure 4.8: Smoothed normalized $C_{G D} ; L_{e f f}=2.3 \mu m \ldots \ldots \ldots \ldots \ldots \ldots$

Figure 4.9: Smoothed normalized $C_{G D} ; L_{e f f}=1.0 \mu m \ldots \ldots \ldots \ldots \ldots \ldots$

Figure 4.10: Smoothed normalized $C_{G S} ; L_{e f f}=49.3 \mu m \ldots \ldots \ldots \ldots \ldots \ldots$

Figure 4.11: Smoothed normalized $C_{G S} ; L_{e f f}=2.3 \mu m \ldots \ldots \ldots \ldots \ldots \ldots \ldots$

Figure 4.12: Smoothed normalized $C_{G S} ; L_{e f f}=1.0 \mu m \ldots \ldots \ldots \ldots \ldots \ldots . \ldots 6$

Figure 4.13 : Smoothed normalized $C_{G B} ; L_{e f f}=2.3 \mu m \ldots \ldots \ldots \ldots \ldots \ldots \ldots$

Figure $4.14:$ Normalized $C_{G G} ; V_{D S}=0.1 V \ldots \ldots \ldots \ldots \ldots \ldots \ldots \ldots \ldots \ldots$

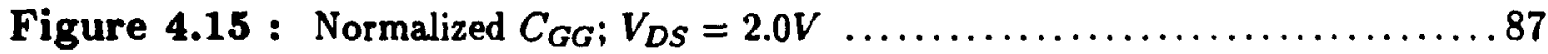

Figure 4.16 $:$ Normalized $C_{G G} ; V_{D S}=4.0 \mathrm{~V} \ldots \ldots \ldots \ldots \ldots \ldots \ldots \ldots \ldots \ldots$

Figure 4.17 : Normalized $C_{G D} ; V_{D S}=0.15 V \ldots \ldots \ldots \ldots \ldots \ldots \ldots \ldots \ldots \ldots \ldots$

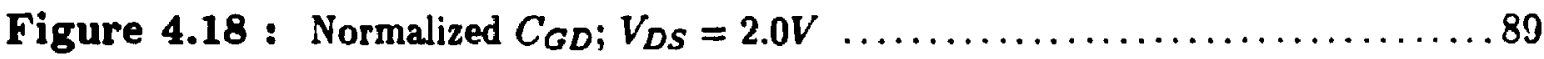

Figure 4.19 : Normalized $C_{G D} ; V_{D S}=4.0 \mathrm{~V} \ldots \ldots \ldots \ldots \ldots \ldots \ldots \ldots \ldots \ldots$

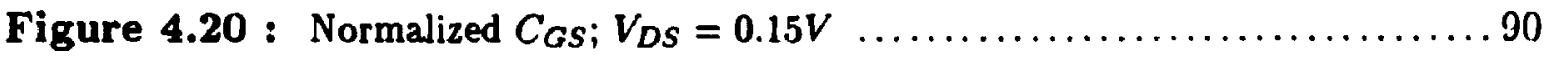

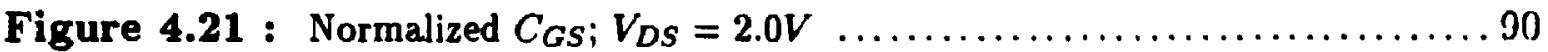

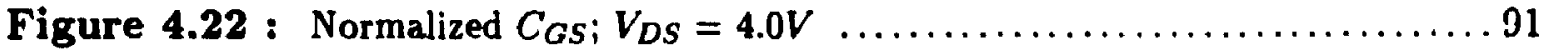

Figure 5.1 : Illustration showing the division of the channel into three regions, according to the analytical model (AM): the 2D space charge regions of the source and drain, and the reduced ID region intersect-

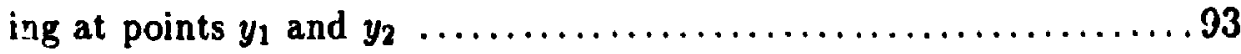


Figure 5.2 : Illustration showing the treatment of the drain (and source) as cylindrical step junctions according to the CJM

Figure 5.3: $\psi_{2}$-distributinn of the source and drain space charge regions, obtained from the CJM for two drain voltages, ignoring the influence of the gate

Figure 5.4 : Parabola used to estimate $\psi_{0}$ in the reduced 1D channel region, $y_{1} \leq y \leq y_{2}$

Figure 5.5: $\quad \psi$-distribution from LCT and QA; $V_{D S}=2.0 \mathrm{~V}$

Figure 5.6 : Distributions of $\psi_{s m}, \psi_{s a t}, \psi_{s s} ; V_{D S}=2.0 \mathrm{~V}$ 100

Figure 5.7 : $\psi_{s}$-distributions from the $A M$ at $V_{D S}=2.0 \mathrm{~V} ; L_{e f f}=2.3 \mu \mathrm{m}$

Figure 5.8: $\quad C_{G D} \sim V_{G S}$ from the $A M ; L_{e f f}=2.3 \mu m$ and $L_{e f f}=1.0 \mu m$

Figure 5.9 : Difference between two $\psi_{\text {. }}$-distribution for different drain voltages;

$L_{c /}=1.0 \mu m$

Figure 5.10 : Distributions of $Q_{G}$ for the 1D channel region; $L_{\text {ef } f}=2.3 \mu \mathrm{m}$ and $L_{\text {eff }}=1.0 \mu \mathrm{m}$

Figure 5.11 : Distributions of $Q_{G}$ for the drain space charge region; $L_{e f f}=2.3$ $\mu m$ and $L_{\text {eff }}=1.0 \mu m$ 106

Figure 5.12(a) : Normalized $C_{G D}$ obtained from MINIMOS and the AM;

$L_{e f f}=2.3 \mu \mathrm{m}$

Figure 5.12(b) : Normalized $C_{G D}$ obtained from MINIMOS and the $A M$;

$L_{e f f}=1.0 \mu \mathrm{m}$

Figure 5.13 : 4 -distributions obtained from simulation and the AM;

$$
L_{\text {eff }}=2.3 \mu \mathrm{m}
$$

Figure 5.14: $C_{G D} \sim V_{G S} ; L_{e f f}=1.0 \mu \mathrm{m}$ 


\section{List of Tables}

Table 3.1.1 : Comparison of $Q_{G}$ determined by various methods. $L_{\text {eff }}$ $=49.3 \mu \mathrm{m} V_{D S}=2 . n V, V_{S B}=0 V ;$ Differences taken with respect to $\psi_{0}$ (MINIMOS)

Table 3.1.2 : Comparison of $Q_{G}$ determined by various methods. $L_{\text {eff }}$ $=2.3 \mu \mathrm{m} ; V_{D S}=2.0 \mathrm{~V}, V_{S B}=0 \mathrm{~V} ;$ Differences taken with respect to $\psi_{\text {s.(MINIMOS) }}$

Table 3.2 : Comparison of $\mathcal{E}_{s i}$ by MINIMOS and long channel theory $L_{e f f}=49.3 \mu \mathrm{m} ; V_{D S}=2.0 \mathrm{~V}, V_{S B}=0 \mathrm{~V}$

Table 3.3 : Comparison of $\mathcal{E}_{\text {si }}$ by MINIMOS and potential differencing.

$L_{\text {eff }}=49.3 \mu \mathrm{m} ; V_{G S}=3.35 \mathrm{~V}, V_{D S}=2.0 \mathrm{~V}, V_{S B}=0 \mathrm{~V}$;

Saturation .46

Table 3.4: Comparison of $\mathcal{E}_{\text {ox }}$ and $\mathcal{E}_{\text {si }} . L_{\text {eff }}=49.3 \mu \mathrm{m} ; V_{G S}=3.35 \mathrm{~V}$,

$V_{D S}=2.0 \mathrm{~V}, V_{S B}=0 \mathrm{~V} ;$ Saturation

Table 3.5 : Comparison of $Q_{G}$ values. $L_{e f f}=49.3 \mu \mathrm{m} ; V_{D S}=2.0 \mathrm{~V}$

$V_{S B}=0 \mathrm{~V}$

Table 4.6 : Comparison of $C_{G G}$ evaluated from simulation without grid-

freeze and from Figure 4.5. $L_{e f f}=1.0 \mu \mathrm{m}$

Table 4.7 : Comparison of $C_{G D}$ values from Figure 4.17, separate simulations and LCT at $V_{G S}=6.0 \mathrm{~V}, V_{D S}=0.15 \mathrm{~V}$

Table $4.8: \quad C_{G D} ; V_{G S}=1.0 \mathrm{~V}$ 76

Table 4.9 : Normalized $C_{G B}$ values obtained from independent simulations for $L_{e f f}=49.3 \mu \mathrm{m} ; V_{G S}=0.4 \mathrm{~V}$ .79 


\section{Notation}

$\mathrm{AM}$

CAD

C.JM

$C_{i}$

$\overline{C_{n}}$

$C_{o}$

$C_{V}$

$C_{G G}$

$C_{G D}, C_{D G}$

$C_{G S}, C_{S G}$

$C_{G B}, C_{B G}$

$C_{D D}$

$C_{D S}, C_{S D}$

$C_{D B} \cdot C_{B D}$

$C_{s S}$

$C_{S B}, C_{B S}$
Analytical Model.

Computer Aided Design.

Cylindrical Step Junction Model; Solution of the 1D radial Poison's equation using cylindrical coordinates.

Capacitance component associated between terminals $i$ and $j$.

$=\frac{1}{2}\left(C_{n+1}+C_{n}\right) ;$ The average capacitance value over the time interval $\left(t_{n+1}-t_{n}\right)$.

$=L_{e f f} \cdot W \cdot \epsilon_{o x} / T_{o x} ;$ The gate oxide capacitance of the device.

$=C\left(\frac{1}{2}\left(V_{n+1}+V_{n}\right)\right) ;$ Capacitance value at the average voltage of the time interval $\left(t_{n+1}-t_{n}\right)$.

Gate self-capacitance.

Gate to drain capacitance component and its reciprocal.

Gate tu source capacitance component and its reciprocal.

Gate to bulk capacitance component and its reciprocal.

Drain self-capacitance.

Drain to source capacitance component and its reciprocal.

Drain to bulk capacitance component and its reciprocal.

Source self-capacitance.

Source to bulk capacitance component and its reciprocal. 
$C_{B B} \quad$ Bulk self-capacitance.

$\epsilon_{o}$

$\epsilon_{\text {ox }}$

$\epsilon_{a i}$

$\mathcal{E}_{\text {ox }}$

$\mathcal{E}_{s i}$

$\psi_{s}$

$\psi_{b o}$

$\psi_{s s}$

$\psi_{s d}$

$\psi_{s m}$

$\psi_{\text {sat }}$

GCA

$i_{G}$

$i_{D}$

$i_{S}$

$i_{B}$

$i(t)$

Permitivity of free space.

Permitivity of the oxide.

Permitivity of the silicon.

Transverse electric field inside the oxide.

Transverse electric field at the silicon surface.

The potential on the surface of the device.

Built-in potential across the metallurgical junction.

$\psi_{s}$ at $y=y_{1}$, defined by the AM as the potential at the edge of the $2 \mathrm{D}$ source space charge region.

$\psi_{\text {s }}$ at $y=y_{2}$, defined by the AM as the potential at the edge of the 2D drain space charge region.

The maximum surface potential in the channel, at which the mobile charge density is zero.

$=\psi_{s s}+\frac{\psi_{s m}-\psi_{s e}}{1+\frac{\psi_{g x}}{L}} ;$ semi-empirical saturation condition defining the surface potential at $y=y_{2}$ according to the AM.

Gradual Change Approximation; 1D theory which assumes $\mathcal{E}_{T} \gg$ $\mathcal{E}_{L}$, where $\mathcal{E}_{T}$ is the transverse field and $\mathcal{E}_{L}$ is the latcral field, everywhere inside the device channel surface.

Charging current of the gate terminal.

Charging current of the drain terminal.

Charging current of the source terminal.

Charging current of the bulk terminal.

The transient current component. 
$i_{i}$

$I_{T}$

$I_{o}$

$I_{D}(t)$

$I_{S}(t)$

$L_{\text {elf }}$

LCT

$L g$

LD

MD

MINIMOS

MOSFET

NT

PISCES-II

QA

$Q_{G}$

$Q_{B}$

$Q_{i}$

$Q_{M}$

$Q_{M D}$
The charging component of the $i^{\text {th }}$ terminal.

The dc transport current between the source and drain terminals.

The steady state current component associated with each terminal.

The total drain current including the dc and ac components.

The total source current including the dc and ac components.

The effective channel length of the device.

Long Channel Theory.

The gate length defined by MINIMOS.

Large Device; $L_{e f f}=49.3 \mu \mathrm{m}$.

Medium Device; $L_{e f f}=2.3 \mu m$.

2D numerical simulator for the plannar MOS transistor.

Metal Oxide Semiconductor Field Effect Transistor.

Northern Telecom.

2D numerical device simulation package.

Quadratic approximation model used to estimate the $\psi_{s}(y)$ in the reduced 1D channel region.

The total gate charge.

The total charge in the bulk of the device.

The total charge associated with the $i^{\text {th }}$ terminal.

The total mobile charge in the channel.

The total mobile charge in the channel associated with the drain. 
The total mobile charge in the channel associated with the source.

$Q_{m}$

The mobile charge density.

$S C D$

Short channel device(s).

SCE

Short channel effect(s).

SD

Short Device; $L_{e f f}=1.0 \mu m$.

$S_{i} O_{2} / S_{i} \quad$ Boundary between the oxide and the silicon surface in the channel of the MOSFET.

SOS

Silicon-on-Sapphire.

$T_{o x}$

Oxide thickness of the MOSFET.

$V_{T H}$

Threshold gate voltage.

$v_{i}(t)$

The transient voltage of the $i^{t h}$ terminal.

$V_{i}$

The steady state voltage of the $i^{\text {th }}$ terminal.

$V_{G B}$

The gate to bulk voltage.

$V_{G S}$

The gate to source voltage.

$V_{D S}$

The drain to source voltage.

$V_{S B}$

The source to bulk voltage.

$V_{g s a t}$

The gate voltage at which saturation is reached, for a given drain voltage.

$V_{F B}$

The flat band voltage of the device.

VLSI

Very Large Scale Intergration.

$W_{\text {eff }}$

The effective channel width of the device. 
Note: The footnotes appear in order of call, separately in every chapter. 


\section{Chapter 1 Introduction}

MOS transistors are the most commonly used elements in modern integrated circuits, thus making it imperative to understand and model their behavior fully. The emergence of VLSI has continued to impose new demands on previously accepted knowledge of the behavior of these devices however, requiring understanding of the changing nature of their performance with the reduction in their size. Whereas the physical mechanisms responsible for the changes in the $I \sim V$ characteristics of such short channel devices, operating under static dc bias conditions, have been extensively studied and well understood for several years [1-5], it has been only recently that the dynamic behavior of such devices have received any attention[6-21].

Predicting the dynamic behavior of the short channel MOSFET requires the understanding of the capacitive effects present in such a device, since they are the dominant factor in circuit timing. It is the study of the gate capacitances associated with the short channel MOS transistor via MINIMOS, a 2D numerical device simulator, that is the main object of this thesis.

The scope of transient analysis of MOSFET devices is discussed in Chapter 2, in which the capacitances associated with the intrinsic MOSFET are defined, and various methods for their modelling discussed, with their associated advantages and disadvantages. The concept of stored charge associated with each terminal is defined and the difficulties that arise from the sharing of the stored charge $Q_{M}$ inside the intrinsic channel between the source and drain terminals discussed; possible methods that have been suggested for partitioning $Q_{M}$ explicitly, in terms of its source and drain associated components, for purposes of evaluating the transient terminal currents of the device are indicaled. Finally, a discussion is given of measurement studies of the short channel gate capacitances and of other studies 
by $2 \mathrm{D}$ numerical analysis, providing insight into the mechanisms that influence the short channel gate capacitance behavior.

Chapter 3 is involved with determining the feasibility of MINIMOS as a means of investigating short channel gate capacitances of MOSFET devices. The various output quantities of MINIMOS (i.c. transverse field inside the oxide $\mathcal{E}_{0 x}$ and inside the silicon surface $\mathcal{E}_{\theta i}$, and surface potential $\psi_{0}$ ) are studied to examine their viability as a basis for investigating the charge properties of the MOSFET associated with capacitance. Different techniques of performing simulations used to determine the most viable results are also discussed.

The results for the gate capacitance characteristics derived from MINIMOS simulations are presented and discussed in Chapter 4, in which an analysis of the accuracy of the characteristics and methods of smoothing the data derived from simulations is given. The features of each of the gate capacitances obtained from MINIMOS simulation, are analyzed and discussed for devices of three different channel lengths, to determine the influences of short channel effects on the capacitance characteristics.

The determination of gate capacitances by 2D numerical simulation, while appropriate for studies of capacitance properties as such, is obviously inadmissible in the context of modelling for practical circuit simulation purposes, from considerations of computing time. Chapter 5 discusses the possibility of determining short channel gate capacitance characteristics using an analytical approach in terms of an approximate representation of the silicon surface potential distribution, which includes the influence of the source and drain space charge regions. The results of the analytical model are then analyzed and compared to those of MINIMOS, and suggestions for possible improvement of the model are made, which could lead to a practical model for predicting actual 2D effects for circuit simulation applications.

Finally, conclusions on the work of the thesis are given in Chapter 6 along with suggestions for further studies. 


\section{Chapter 2 \\ Transient Analysis of the MOSFET}

In order to predict the transient response of a circuit it is necessary to have a dynamic model for each of the circuit elements involved. For transistor circuits such models should focus on the capacitive effects of each element, these being one of the dominant factors in circuit timing.

\subsection{Dynamic Analysis of the MOS Transistor}

By far the most commonly used element in modern integrated circuits today is the MOS transistor, the dynamic behavior of which is of interest here. Figure 2.1 shows the MOS transistor divided into two physical regions whose capacitive effects can be treated separately: first, the intrinsic region which is responsible for the basic device mechanism, the dynamic effects of which, involving charge storage, are capacitive; and second, the extrinsic region, which may be modelled by fixed or junction capacitances (i.e. overlap, lateral diffusion, $\mathbf{p}-\mathbf{n}$ junction, sidewall and other parasitics which can limit overall performance). It is the modelling of the capacitive effects for the intrinsic MOSFET structure that is investigated in this thesis.

In order to simplify the dynamic analysis of the MOS transistor, the following ideal assumptions must be made: there is zero leakage current flow in the gate and bulk (i.e. the transport current flow is entirely between the source and drain terminals with negligible static gate and substrate currents) and quasi-static conditions apply. The quasi-static operation of a device is defined to be one where the variations of the terminal voltages $v_{i}(t)$ is sufficiently slow so that the calculated charges per unit area at any time $t$ are assumed to be identical to those that would 


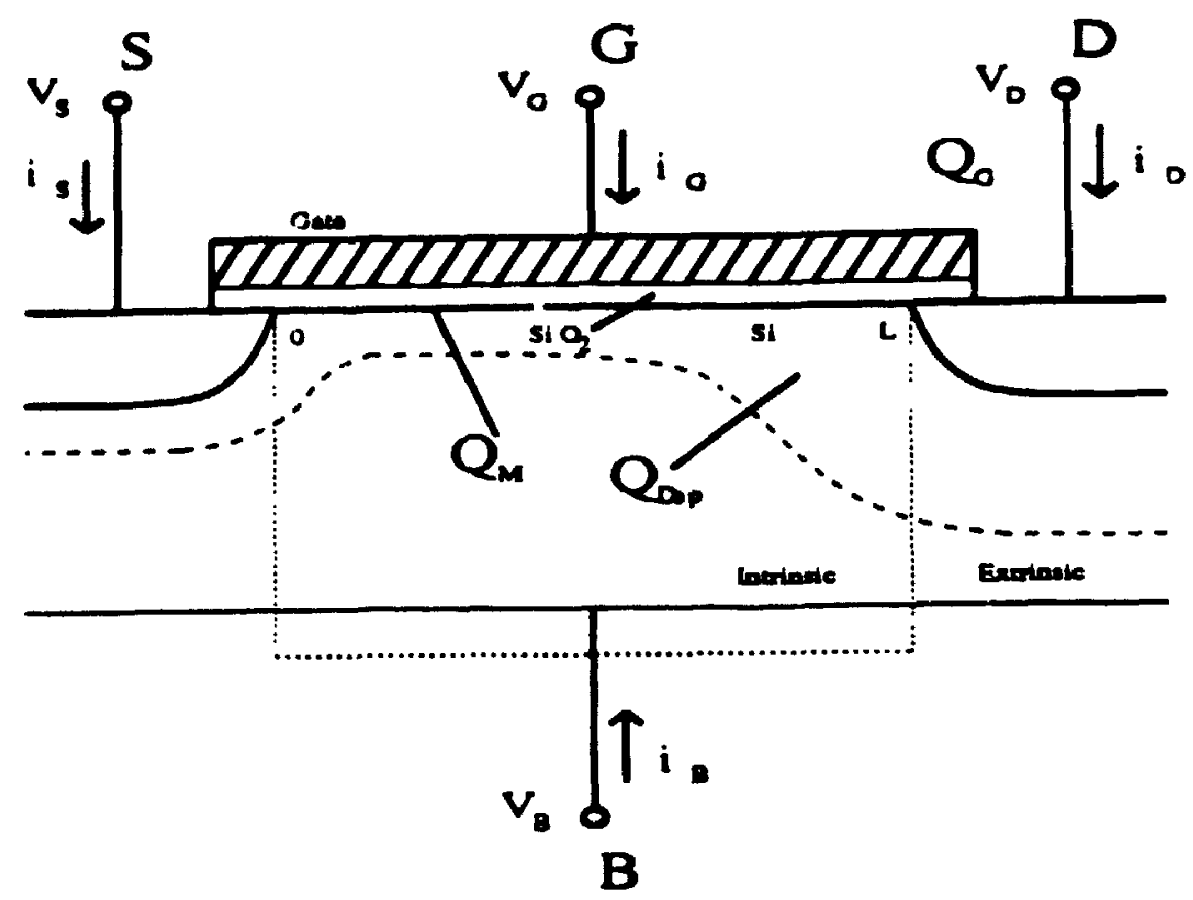

Figure 2.1 Structure of MOSFET showing the intrinsic and extrinsic regions.

be calculated if de steady-state conditions were applied instead, i.e. $v_{i}(t)=V_{1}$, where lower case letters represent time-dependent quantities and upper case letters represent dc quantities.

The four terminal currents of the MOSFET may therefore be defined as

$$
\begin{gathered}
I_{G}=i_{G}=\frac{d Q_{G}}{d t} \\
I_{B}=i_{B}=\frac{d Q_{B}}{d t} \\
I_{S}=i_{S}+I_{T}=\frac{d Q_{M S}}{d t}+I_{T}=\left[I(y, t)_{d i f f}+I(y, t)_{d r i f t}\right]_{y=0} \\
I_{D}=i_{D}-I_{T}=\frac{d Q_{M D}}{d t}-I_{T}=-\left[I(y, t)_{d i f f}+I(y, t)_{d r o f t}\right]_{y=L}
\end{gathered}
$$


where $Q_{G}, Q_{B}, Q_{M S}, Q_{M D}$ are the stored gate, bulk, source and drain charges in the intrinsic device, and $i_{G}, i_{B}, i_{S}, i_{D}$ represent their respective charging currents. with $I_{T}$ being the transport current. The total mobile charge in the device is given by

$$
Q_{M}=Q_{M S}+Q_{M D}
$$

The stored charges $Q_{G}$ in the gate and $Q_{\Gamma}$ in the bulk can be obtained with little difficulty, but $Q_{M S}$ and $Q_{M D}$ represent the stored portion of the channel charge that is associated with the source and drain respectively ${ }^{1}$ which is much more difficult to determine. A method for partitioning the mobile channel charge $Q_{M}$ into its source and drain controlled parts $Q_{M S}$ and $Q_{M D}$ respectively must be developed $^{2}$ in order to define the source and drain charging currents. However one decides to partition $Q_{M}$ though, the technique will remain approximate. The only physically correct statement that can be made is that the sum of the soirce and drain currents represents the total current entering the channel which will change $Q_{M}$; that is

$$
I_{D}(t)+I_{S}(t)=\frac{d Q_{M}}{d t}
$$

For purposes of simulation each terminal of a device must be considered separately and the potential at each node is taken with respect to an arbitrary reference. There are corresponding charges and currents associated with each noxle as well. Thus defined, these quantities are shown for the simplified case of a non-linear two terminal capacitor in Figure 2.2.

1 This is a loose and physically incorrect description of $Q_{M S}$ and $Q_{M D}$. These quantities should not be assigned any precise physical significance; a method of defining them will be discussed in Section 2.3.

2 Various methods have been proposed to define $Q_{M S}$ and $Q_{M D}$ as being stored charges by partitioning $Q_{M}[9-12]$. They will be discussed in detail in Section 2.3.1. For now however, the existence of these quantities as stored must be assumed. 


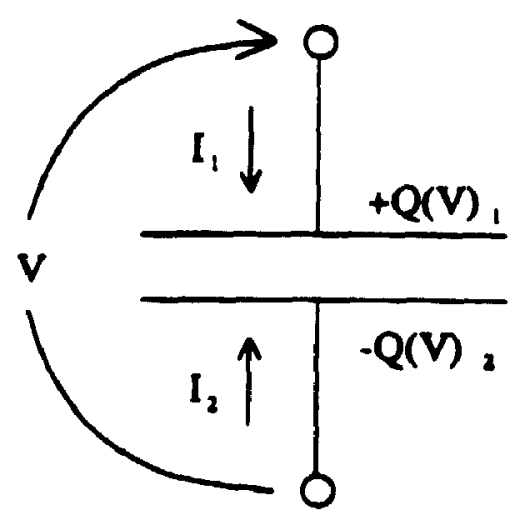

Figure 2.2 A non-linear two-terminal capacitor with corresponding quantities defined for simulation in a circuit simulation environment.

An important fact must be noted at this point about the physical nature of a multi-terminal device such as the MOS transistor. The charge on every terminal is a function of all the terminal voltages of the device; that is to say, the charging current on each terminal is subject to the capacitance coupling effects with every terminal of the device. Due to the geometrical structure of the MOSFET, and the dependence of the physical (and thus electrical) properties on the state of the applied voltages at any given time, the capacitances associated with the intrinsic device are both non-linear and time-dependent and thus give rise to its complex transient behavior. The charge on every terminal is a non-linear function of all terminal voltages and therefore the capacitance is a function of voltage and may vary with time that is, the charging (dynamic) component of current $i_{i}$ entering device terminal $i$ and having associated stored charge $Q_{i}$ in a multi-terminal device, is

$$
i_{i}=\frac{d Q_{i}}{d t}=\sum_{j} \frac{\partial Q_{i}}{\partial v_{j}} \frac{d v_{j}}{d t}
$$

for all device terminals 3 , where all $v$, are with respect to some arbitrary reference. 
The charging currents flowing into the four terminal MOSFET are functions of the potentials at each node as mentioned above; by the use of the chain rule, the four charging currents of the device may therefore be defined as

$$
\begin{gathered}
i_{G}=\frac{d Q_{G}}{d t}\left(v_{G}, v_{D}, v_{S}, v_{B}\right)=\frac{\partial Q_{G}}{\partial v_{G}} \frac{d v_{G}}{d t}+\frac{\partial Q_{G}}{\partial v_{D}} \frac{d v_{D}}{d t}+\frac{\partial Q_{G}}{\partial v_{S}} \frac{d v_{S}}{d t}+\frac{\partial Q_{G}}{\partial v_{B}} \frac{d v_{B}}{d t} \\
i_{D}=\frac{d Q_{D}}{d t}\left(v_{G}, v_{D}, v_{S}, v_{B}\right)=\frac{\partial Q_{D}}{\partial v_{G}} \frac{d v_{G}}{d t}+\frac{\partial Q_{D}}{\partial v_{D}} \frac{d v_{D}}{d t}+\frac{\partial Q_{D}}{\partial v_{S}} \frac{d v_{S}}{d t}+\frac{\partial Q_{D}}{\partial v_{B}} \frac{d v_{B}}{d t} \\
i_{S}=\frac{d Q_{S}}{d t}\left(v_{G}, v_{D}, v_{S}, v_{B}\right)=\frac{\partial Q_{S}}{\partial v_{G}} \frac{d v_{G}}{d t}+\frac{\partial Q_{S}}{\partial v_{D}} \frac{d v_{D}}{d t}+\frac{\partial Q_{S}}{\partial v_{S}} \frac{d v_{S}}{d t}+\frac{\partial Q_{S}}{\partial v_{B}} \frac{d v_{B}}{d t} \\
i_{B}=\frac{d Q_{B}}{d t}\left(v_{G}, v_{D}, v_{S}, v_{B}\right)=\frac{\partial Q_{B}}{\partial v_{G}} \frac{d v_{G}}{d t}+\frac{\partial Q_{B}}{\partial v_{D}} \frac{d v_{D}}{d t}+\frac{\partial Q_{B}}{\partial v_{S}} \frac{d v_{S}}{d t}+\frac{\partial Q_{B}}{\partial v_{B}} \frac{d v_{B}}{d t}
\end{gathered}
$$

These charging currents may be represented by the following mathematically elegant vector form

$$
\left(\begin{array}{l}
i_{G} \\
i_{D} \\
i_{S} \\
i_{B}
\end{array}\right)=\left(\begin{array}{llll}
+C_{G G} & -C_{G D} & -C_{G S} & -C_{G B} \\
-C_{D G} & +C_{D D} & -C_{D S} & -C_{D B} \\
-C_{S G} & -C_{S D} & +C_{S S} & -C_{S B} \\
-C_{B G} & -C_{B D} & -C_{B S} & +C_{B B}
\end{array}\right) \frac{d}{d t}\left(\begin{array}{c}
v_{G} \\
v_{D} \\
v_{S} \\
v_{B}
\end{array}\right)
$$

or more generally

$$
i_{i}=\delta_{i j} C_{i j} \frac{d v_{j}}{d t}
$$

where

$$
\delta_{i j}= \begin{cases}+1, & i=j \\ -1, & i \neq j\end{cases}
$$


and $C_{i j}$ represents the full capacitance matrix of the intrinsic MOSFET.

This mathematical representation may be simplified further by realizing the physical conditions that must be imposed:

1. The net charge and the sum of the charging currents in the intrinsic device must be zero; that is

$$
\begin{gathered}
Q_{G}+Q_{B}+Q_{M}=0 \\
i_{G}+i_{D}+i_{S}+i_{B}=0
\end{gathered}
$$

Mathematically this may be interpreted as

$$
\sum_{j} C_{i j}=0
$$

i.e. the sum of the columns in the capacitance matrix must be zero.

2. If the terminal voltages all increase or decrease by the same rate, the charging current must be zero, which implies

$$
\sum_{i} C_{i j}=0
$$

i.e. the sum of the rows in the capacitance matrix must be zero.

These conditions therefore allow for nine independent $C_{i j}$ parameters which corresponds to three degrees of freedom. From a physical point of view this may be interpreted to mean ouite simply that any terminal may serve as a reference.

\subsection{Dynamic Modelling Methods}

The object of transient analysis is to determine the nodal relationships of current and voltage in a circuit as functions of time, with soiutions obiained at discrete timepoints. In order to achieve this it is necessary to have a model of each of the circuit elements that represents their dynamic behavior. This implies focusing on 
the capacitive effects associated with each element, the concern here being with the dynamic analysis of the MOS transistor in particular.

The dynamic representation of the MOSFET is basically complex, involving time-dependent, distributed stored charges related non-linearly to the terminal voltages. For modelling purposes it is convenient to separate the time-dependent current relationship for given terminals into a steady-state and transient component ${ }^{3}$

$$
I(t)=I_{o}(v)+i(t)
$$

where $I_{0}$ is a function of the instantaneous de terminal voltages and must be evaluated under dc conditions. The challenge remains with the transient current component $i(t)$, which is zero under steady state conditions. This current component is capacitive in nature; that is

$$
i_{i}(t)=\frac{d Q_{i}}{d t}=\sum_{j} C_{i}\left(v_{j}\right) \frac{d v_{j}}{d t}
$$

where the charge $Q_{i}$ and/or capacitance $C_{i}$ must be evaluated.

\subsubsection{Numerical Methods of Modelling Capacitances}

For simplicity, the discussion will initially be in terms of the two terminal case. The results and conclusions are then generalized for the multi-terminal case.

\section{i. Capacitance-Based Method}

For the purposes of nodal analysis, (2.14) would require the linearization of the non-linear elements and their discretization in time. This implies an approximation

3 For a MOSFET, $I_{0}(v)$ is only present when it represents the transport current between the source and drain, which is a function of the dc voltages. In an ideal device, such as is assumed here, there is no dc component of current flow into the gate and bulk, thus the total gate and bulk currents are associated with the changing gate and bulk charge respectively. 
of the solution algorithm (i.e. of the derivatives and integral quantities) at discrete timepoints. One such general approximation may be given for the two terminal case by

$$
\int_{t_{n}}^{t_{n+1}} i d t=C\left(V_{n+1}-V_{n}\right)
$$

where the subscripts denote the timepoints at which the corresponding quantity is evaluated.

Numerical integration techniques such as the trapezoidal approximation may be used to evaluate the left hand side of (2.15) as

$$
\int_{t_{n}}^{t_{n+1}} i d t \simeq \frac{k}{2}\left(i_{n+1}+i_{n}\right)
$$

where $k$ is the timestep defined as

$$
k=t_{n+1}-t_{n}
$$

Such an integration technique is approximate by definition; there will always be some error even for linear circuits, where the amount of error is proportional to the size of the timestep $k$. The error for a given timestep however will depend on the circuit, there being no general relation between the associated error and the timestep $k$.

The integration of the right hand side of (2.15) for a non-linear device such as a MOSFET, requires that capacitance $C$ be linearized over the voltage interval. This can take any one of the following forms

$$
\int_{t_{n}}^{t_{n+1}} i d t \simeq\left\{\begin{array}{l}
C_{n}\left(V_{n+1}-V_{n}\right) \\
C_{n+1}\left(V_{n+1}-V_{n}\right) \\
C_{n}\left(V_{n+1}-V_{n}\right) \\
C_{\bar{V}}\left(V_{n+1}-V_{n}\right)
\end{array}\right.
$$


where $\overline{C_{n}}$ is the capacitance averaged over the interval, that is

$$
\overline{C_{n}}=\frac{1}{2}\left(C_{n+1}+C_{n}\right)
$$

and $C_{\nabla}$ represents the capacitance value at the average voltage in the interval, i.e. $C\left(\frac{1}{2}\left(V_{n+1}+V_{n}\right)\right)$. In any case, calculating for the change in charge over a voltage interval in a non-linear device using constant values for capacitance, no matter how close the approximation, will introduce an error. When the nodal equation is calculated over several timesteps, this error term $\epsilon_{n}$ will tend to accumulate, resulting in an apparent non-conservation of charge; that is

$$
\int_{t_{n}}^{t_{n+1}} i d t \simeq Q\left(V_{n+1}\right)-Q\left(V_{n}\right)+\sum_{k=1}^{h} \epsilon_{k}
$$

The non-conservation of charge of the capacitance-based method of calculating charge differences based on such a nodal relationship may present serious errors when sim !lating the transient response of certain types of circuits, such as dynamic memories and silicon-on-sapphire (SOS) transistors. However, this conventional technique has been implemented successfully in simulating the transient behavior of most practical circuits.

The simple form of the nodal expression presented thus far basically describes the case of a two-terminal capacitor, which is characterized by a scalar $C$. Likewise there is a system of $m$ equations that may represent any $m$ terminal device. In vector form, the behavior of such a device would be characterized by an $m \times m$ capacitance matrix. Equation (2.11) gives the capacitance matrix that characterizes the dynamic behavior of the four-terminal MOSFET.

\section{ii. Charge-Based Method}

Charge is not conserved when the calculated stored charge in each node is not equal to the integrated net current flowing into it. This is the case when the 
stored charge in the node is obtained using an approximate value of capacitance as described in any of the methods given by equation (2.17).

A method for calculating the transient currents directly as the derivatives of the charges has been proposed[12].

$$
\int_{t_{n}}^{t_{n+1}} i d t=\int_{Q_{n}}^{Q_{n+1}} d Q=Q_{n+1}-Q_{n}
$$

This relationship is analogous to $(2.17)$, but the right hand side does not make any approximations in order to determine the charges at different timesteps; charge is computed directly, therefore the right-hand side represents the exact integral of charge and hence charge is conserved.

The left-hand side of (2.19) must be approximated by converting the differential quantities into differences, as given by (2.16) and the accuracy will depend upon the sophistication of the numerical integration technique used.

Another advantage of this numerical method is its inherent numerical stability, due to the fact that charge is a smooth function of voltage and does not have any sudden changes in its characteristics, unlike the capacitance in many device models; therefore ensures faster convergence of the numerical iteration procedure.

The basic current-voltage nodal relationship for a two-terminal device may be obtained from (2.19), the unknown charge $Q_{n+1}$ being linearized to give

$$
\int_{t_{n}}^{t_{n+1}} i d t=Q_{n+1}^{i}+\left.\frac{\partial Q}{\partial V}\right|_{V=V_{n+1}^{i}}\left(V_{n+1}^{i+1}-V_{n+1}^{i}\right)-Q_{n}
$$

where the subscripts represent the timepoints and the superscripts denote the iteration steps. Convergence of the solution is attained for each timepoint as the quantity $\left(V_{n+1}^{i+1}-V_{n+1}^{i}\right)$ goes to zero and $Q_{n+1}^{i+1}$ approaches $Q_{n+1}^{i}$.

This relationship may be extended to apply to devices of more than two terminals, provided that the voltages, currents and charges be expressed in vector form 
and the derivatives of charge with respect to voltage be expressed by a capacitance matrix. In the case of the four-terminal MOS transistor, the nodal matrix equation takes the form:

$$
\begin{aligned}
\int_{t_{n}}^{t_{n+1}}\left(\begin{array}{l}
i_{G} \\
i_{D} \\
i_{S} \\
i_{B}
\end{array}\right) d t= & \left(\begin{array}{l}
Q_{G} \\
Q_{D} \\
Q_{S} \\
Q_{B}
\end{array}\right)_{t_{n+1}}^{i}+\left(\begin{array}{llll}
+C_{G G} & -C_{G D} & -C_{G S} & -C_{G B} \\
-C_{D G} & +C_{D D} & -C_{D S} & -C_{D B} \\
-C_{S G} & -C_{S D} & +C_{S S} & -C_{S B} \\
-C_{B G} & -C_{B D} & -C_{B S} & +C_{B B}
\end{array}\right)_{t_{n+1}}^{i} \times \\
& \left(\begin{array}{l}
V_{G}^{i+1}-V_{G}^{i} \\
V_{D}^{i+1}-V_{D}^{i} \\
V_{S}^{i+1}-V_{S}^{i} \\
V_{B}^{i+1}-V_{B}^{i}
\end{array}\right)_{t_{n+1}}-\left(\begin{array}{c}
Q_{G} \\
Q_{D} \\
Q_{S} \\
Q_{B}
\end{array}\right)_{t_{n}}
\end{aligned}
$$

Charge $Q(V)$ is the state variable and is therefore the primary quantity required by the charge-based model for simulation. The capacitances $d Q\left(V_{i}\right) / d V_{j}$ are not important as far as the accuracy of the transient analysis is concerned though they are necessary and need to be completely represented for use in the iteration method. Thus the full capacitance matrix of the intrinsic device must be properly represented in the nodal equation.

Obtaining the capacitance matrix has intuitive advantages as well, since device and circuit behavior are more easily understood in terms of capacitances rather than charges, which tend to have very smooth, uninformative-looking characteristics with respect to voltage. Another problem with charges is that they are difficult to obtain experimentally whereas capacitances are relatively easy to measure and may therefore be used for comparison purposes. For the MOSFET, however, charge relationships are usually easier to derive in device modelling than capacitances.

\subsubsection{Capacitance Matrix}

\section{i. Full Capacitance Matrix}

Modelling the transient terminal currents requires that the stored charges be associated with each terminal of the device. For the MOS transistor therefore, the gate, source, drain and bulk charges are required. 
It is relatively easy to determine the gate and bulk charges $Q_{G}$ and $Q_{B}$, representing the amount of stored charge in the gate electrode and the bulk depletion region respectively. However, finding the source and drain charges $Q_{M S}$ and $Q_{M D}$ is complicated by the fact that they merge to form the channel charge $Q_{M}$. The source and drain, being connected by a conductive channel, interferes with the the definition of the source and drain transient currents $i_{S}$ and $i_{D}$ as independent functions of $Q_{M S}$ and $Q_{M D}$. That is, $Q_{M S}$ and $Q_{M D}$ cannot be interpreted as stored charges that are associated exclusively with the source and drain terminals respectively, due to the charge transfer between the source and drain. The transient source and drain currents are therefore related to the total mobile channel charge by (2.5). This difficulty may be resolved by partitioning the total channel charge $Q_{M}$ into $Q_{M S}$ and $Q_{M D}[9-12]$ which may then be interpreted as stored charges, being suitable for use in the charge-based numerical method. The various methods that have been developed to define $Q_{M S}$ and $Q_{M D}$ as stored charges by partitioning $Q_{M}$ will be discussed further in Section 2.3.1 of this chapter.

Once these charges have been determined, differentiating them with respect to the terminal voltages defines the full capacitance matrix for the MOS transistor as shown in (2.7) - (2.10) and (2.11), having nine independent capacitance terms, whose details and accuracy depend on the accuracy of the derived charges. It should also be noted that two capacitance parameters are defined between each pair of terminals and these capacitances are not generally the same; that is, the terms are not reciprocal. Thus, for example, the capacitance terms between the gate and drain terminals $C_{G D}$ and $C_{D G}$ differ both in value and physical interpretation as shown in Figure 2.3. $C_{G D}$ describes the gate current produced by a change in drain voltage, whereas $C_{D G}$ describes the drain current which results from a change in gate voltage. In the idealized situation of classical long-channel device theory, during saturation $C_{G D}$ is essentially zero since the drain is isolated from the channel and any change cannot affect the gate charge. However, the reverse is not true, i.e. a change in the gate voltage will effect the channel charge, some of which is associated with the drain. Thus $C_{D G}$ will not be ze:o in the saturation region. 


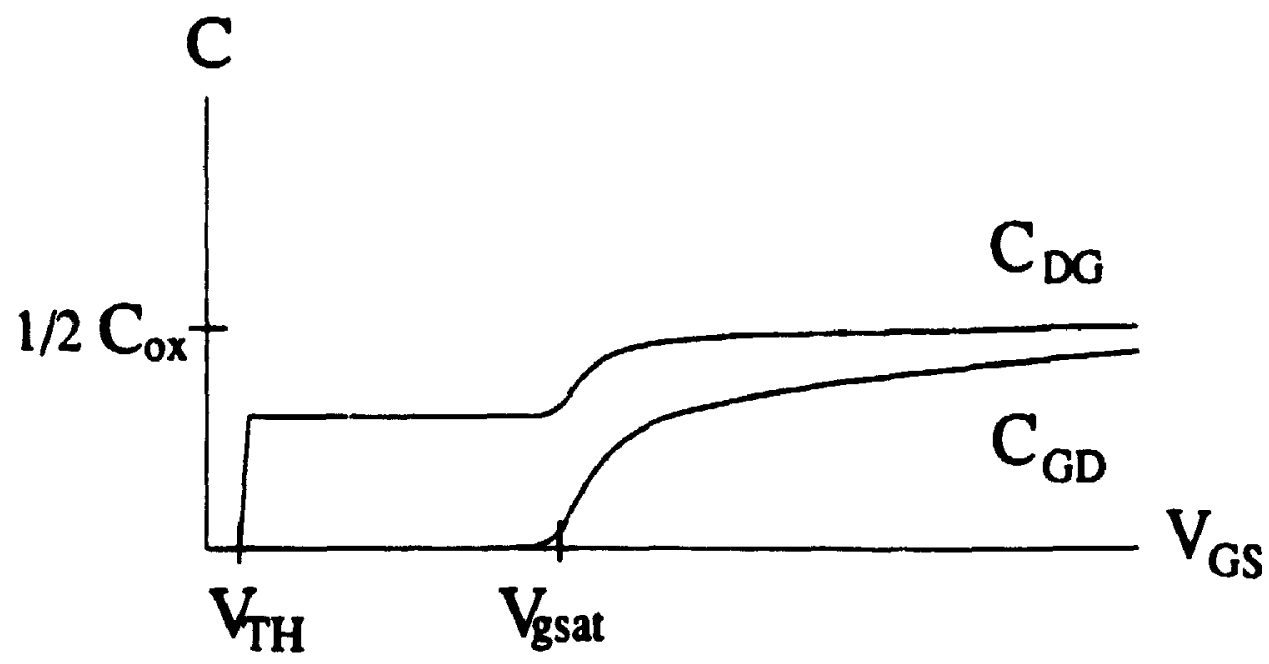

Figure 2.3 Gate-drain capacitances $C_{G D}$ and $C_{D G}$ in MOSFET.

\section{ii. Simplified Gate Capacitance M sdel : Meyer's Model}

The full capacitance matrix, though the most accurate method of determining the transient terminal currents in the MOSFET, is also complicated to determine and more demanding in CPU time in typical circuit simulation applications. In the majority circuit applications of the MOS transistor, the bulk and source terminals are kept at a fixed potential, while the drain and especially the gate vary, determining the various regions of operation of each device.

Practical circumstances therefore impose the need to model the gate capacitances $C_{G D}, C_{G S}$ and $C_{G B}$ over all others, for the sake of simplicity and speed of simulation, the gate charge being the most common and significant varying physical quantity in most practical circuits. Such a model does. in fact, give good approximation to the dynamic performance of the MOSFET for a wide range of practical applications, and is greatly used. Such a representation of the device in terms of only the three gate capacitances, and with corresponding simplification of the capacitance matrix was proposed by Meyer [8]. The result is a symmetrical matrix that can be represented by a network of two-terminal capacitors. This simplified 
model as applied to the MOS transistor is the basis for large-signal models used in most circuit simulations.

The model derivation begins with a network of reciprocal capacitors from which a symmetrical matrix is derived. Meyer's gate capacitance model makes the following assumptions in order to determine the capacitance parameters of the matrix:

1. The total gate charge $Q_{G}$ is calculated as a function of terminal voltages under steady-state conditions. This implies the application of the quasi-static approximation. It is also assumed that current transport is one-dimensional (parallel to the surface) and takes place via a single carrier type. The gradual channel approximation is used to reduce Poisson's equation to one dimension which is further simplified by assuming strong inversion and depletion approximation.

2.

$$
\begin{gathered}
C_{G D}=-\frac{\partial Q_{G}}{\partial V_{D}} \\
C_{G S}=-\frac{\partial Q_{G}}{\partial V_{S}} \\
C_{G B}=-\frac{\partial Q_{G}}{\partial V_{B}} \\
C_{G G}=C_{G D}+C_{G S}+C_{G B}
\end{gathered}
$$

3.

$$
\begin{aligned}
& C_{D S}=C_{D B}=C_{S B}=0 \\
& C_{S D}=C_{B D}=C_{B S}=0
\end{aligned}
$$

4. The capacitance matrix is symmetrical 


$$
C_{D G}=C_{G D}, \quad C_{S G}=C_{G S}, \quad C_{B G}=C_{G B}
$$

The electrical representation of the gate-capacitance method and the resulting capacitance matrix is shown in Figure 2.4(a) and (b), where the variable capacitance symbol used is intended to indicate that the capacitance is voltage-bias dependent.

The gate capacitance parameters are obtained by calculating the gate charge $Q_{G}$ in every region of operation based on given assumptions and approximations while the other capacitance parameters are either approximated from the three gate capacitances, or are disregarded. The gate capacitance characteristics obtained using the Meyer model are shown in Figure 2.5.

The analytical formulation of Meyer's gate capacitance model is subject to errors mainly due to the crude representation of the device physics, the results of which can be seen in Figure 2.5. More refined gate capacitance models have been obtained[12-14] which are based in a more accurate representation of the basic physics, as well as taking greater care to remove any discontinuities in the characteristics which may interfere with the convergence of the iteration procedure.

\subsection{Evaluation of Terminal Currents in Quasi- Static Operation}

The quest to describe the transient behavior of the MOS transistor, such that an accurate method of modelling its dynamic behavior is derived, makes it imperative to properly evaluate the terminal currents of the device. In this section, the physical mechanisms of the intrinsic device under transient conditions are analyzed, in order to properly define the varying quantities affecting the terminal currents.

Quasi-static operation will be assumed to apply everywhere in the discussion, i.e. the variations of the terminal voltages are slow enough so that the charges do not exhibit any noticeable inertia and can be expected to readjust themselves instantaneously. 
18

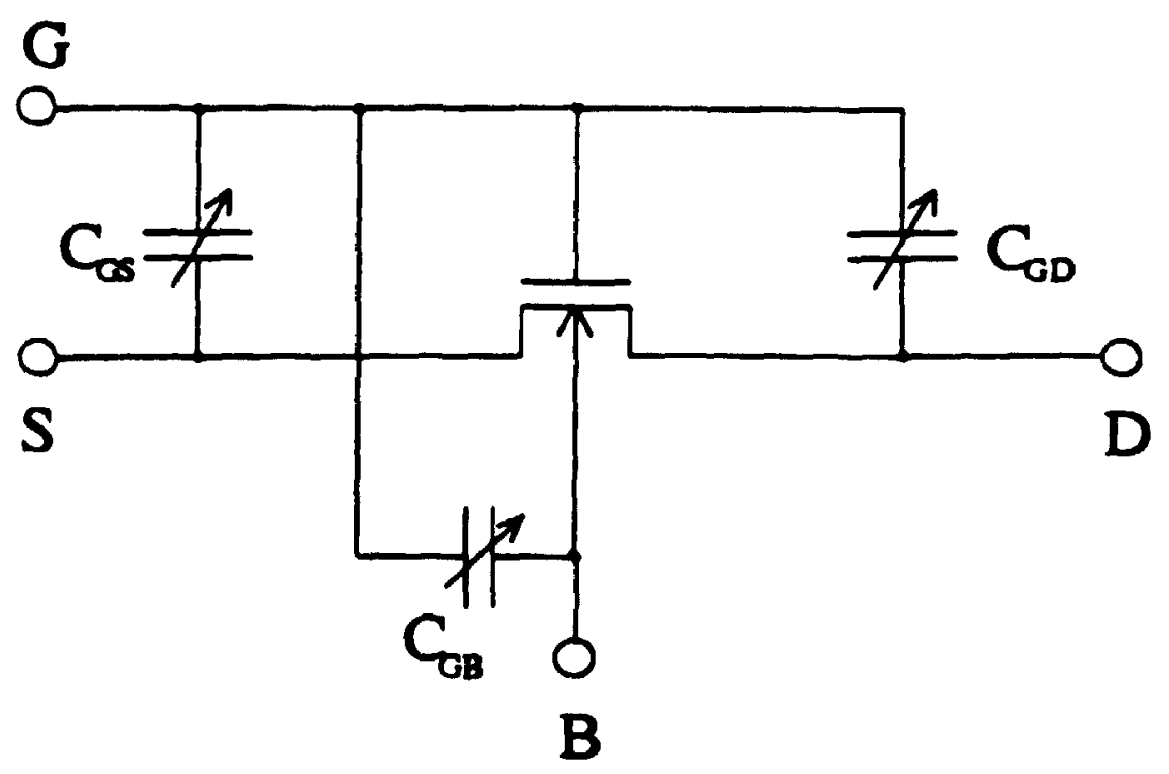

Figure 2.4(a) Circuit representation of Meyer's Model.

$$
\left(\begin{array}{cccc}
C_{G D}+C_{G S}+C_{G B} & -C_{G D} & -C_{G S} & -C_{G B} \\
-C_{G D} & +C_{G D} & 0 & 0 \\
-C_{G S} & 0 & +C_{G S} & 0 \\
-C_{G B} & 0 & 0 & +C_{G B}
\end{array}\right)
$$

Figure 2.4(b) Meyer's simplified capacitance matrix. 

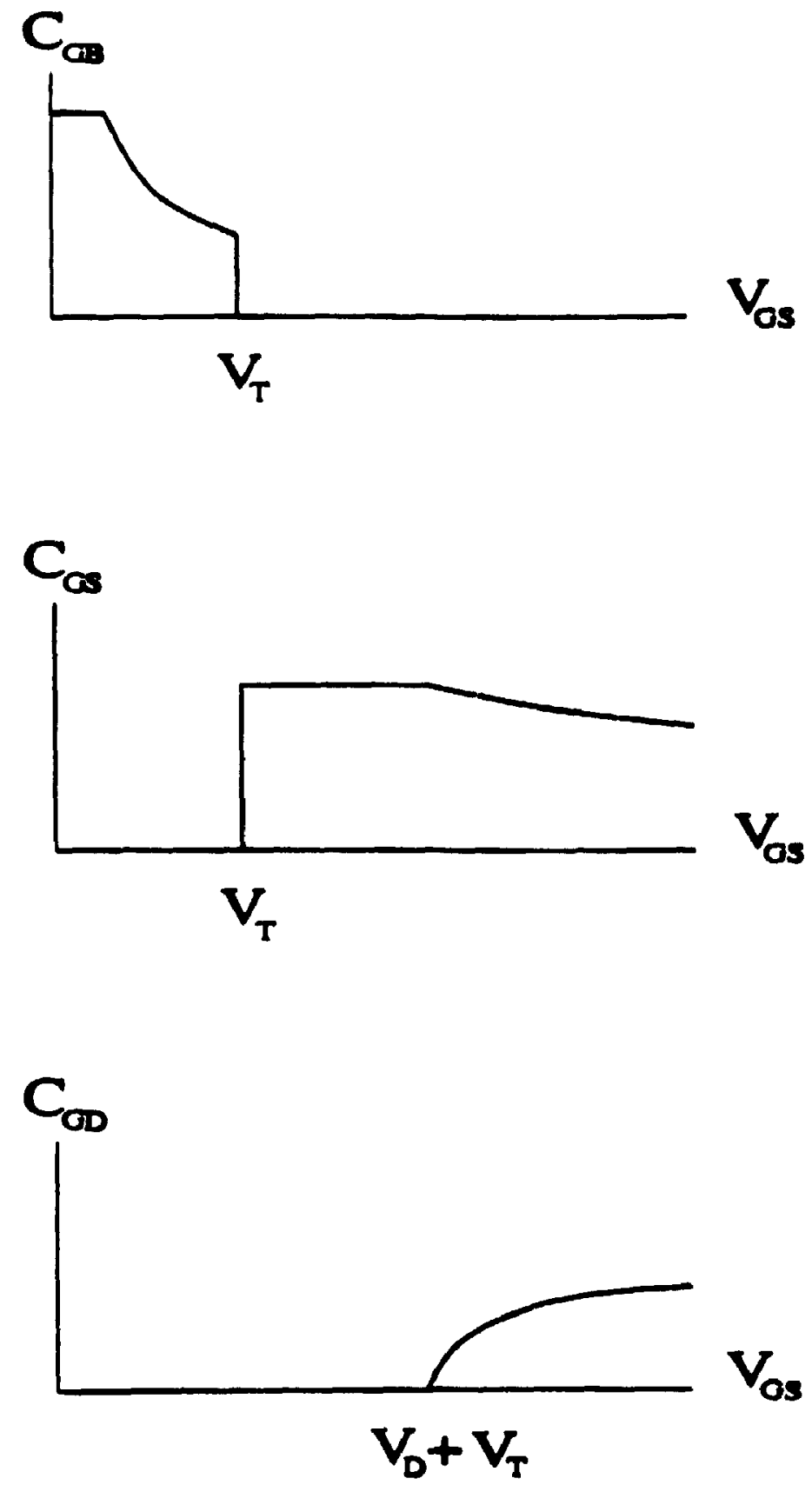

Figure 2.5 $C_{G D}, C_{G S}, C_{G B}$ from Meyer's model. (Note: The voltage $V_{D}$ refers to $V_{D S}$ and $V_{T}$ refers to the threshold gate voltage $\left.V_{T H}\right)$. 
It was mentioned previously, in Sectiors 2.1, and 2.2.2 that in an ideal device with no leakage current in the gate and bulk, the total currents in these regions are associated with changing the gate and bulk charge respectively; thus

$$
\begin{gathered}
I_{G}(t)=\frac{d Q_{G}}{d t} \\
I_{B}(t)=\frac{d Q_{B}}{d t}
\end{gathered}
$$

It was also described by equations (2.3) and (2.4) that the sum of the total source and drain currents entering the channel are associated with the change of the inversion layer charge; that is

$$
I_{D}(t)+I_{S}(t)=\frac{d Q_{M}}{d t}
$$

Given this as a starting point, it is obvious that under steady-state conditions, the total source and drain currents must be equal; i.e. $I_{D}(t)=-I_{S}(t)^{4}$.

It is perhaps best to use an example to better illustrate this point ${ }^{3}$. In order to simplify the analysis, the gate terminal will have varying potential $V_{G}(t)$, while the other terminals will remain at fixed steady-state voltages. It will further be assumed that the voltages are chosen to be such as to enable the device to operate in the linear region, having the source and drain terminals connected by a conducting surface layer of mobile charge. Figure 2.6(a) shows the device in steady-state operation in regions $(a)$ and $(c)$, and during a transient in region $(b)$.

When the gate voltage is increased (Figure 2.6(a)), the magnitude of the mobile channel charge $\left|Q_{M}\right|$ must also increase (Figure 2.6(b)). This increase in the channel charge must be provided to the channel by increasing the rate of electrons entering

$4-I_{S}(t)$ is defined as the measure of the rate at which carriers enter the channel from the source, $I D(t)$ is the measure of the rate at which carrier leaves the drain.

5 From Tsividis[.4], pp. 254-261. 

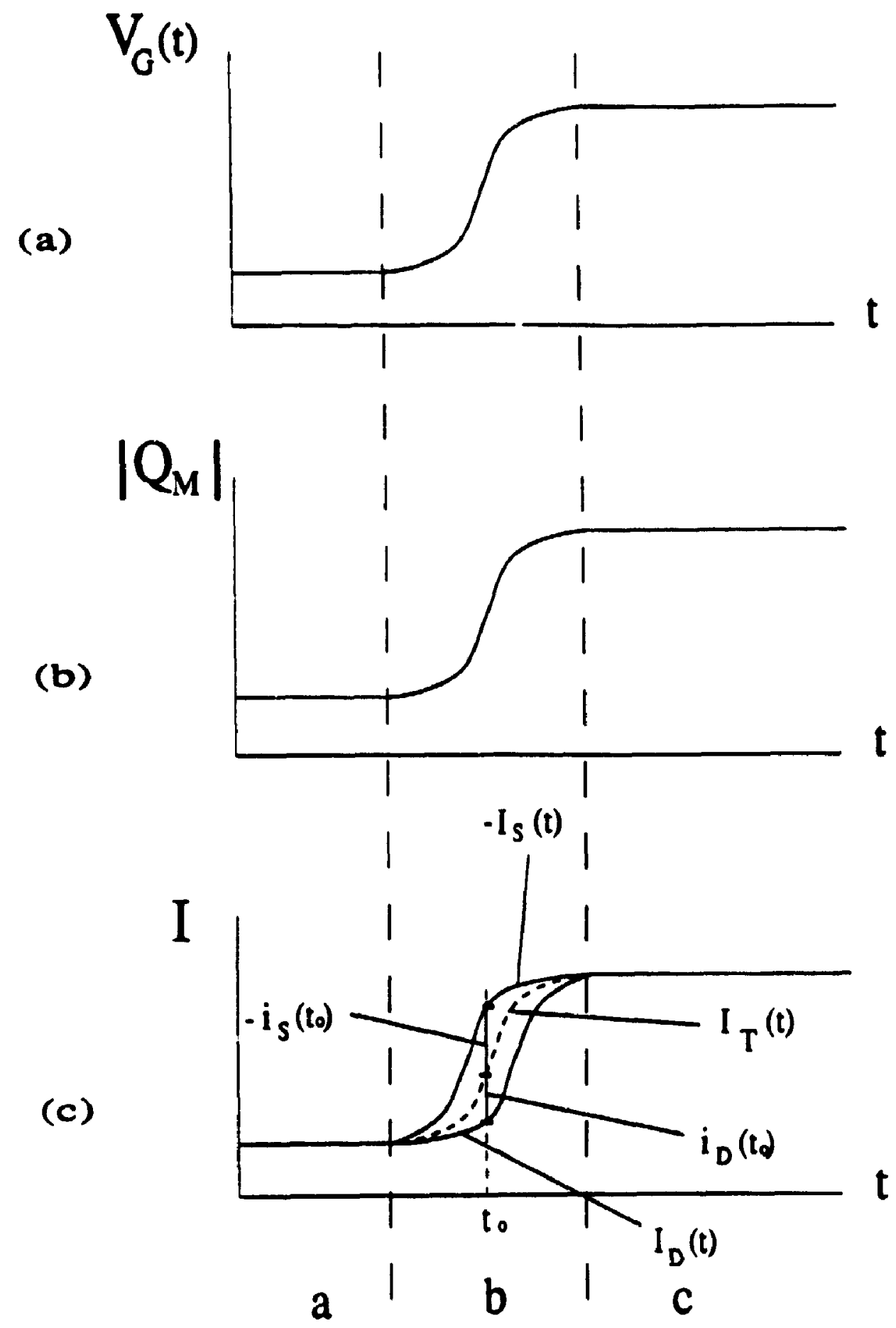

Figure 2.6 Gate voltage waveform (a), causing a change in mobile charge $Q_{M}(b)$, being reflected in the total source and drain current charges ( $c$ ). 
the channel from the source, $-I_{S}(t)$, while the rate of electrons leaving from the drain $I_{D}(t)$, remains unchanged until such time later, when the change in the mobile charge in the channel will have satisfied the electrostatic conditions in response to the increase in gate voltage $V_{G}(t)$ (see Figure 2.6(c)). From (2.24) it follows that the change in mobile charge is given by the difference between the curves of total drain current $I_{D}(t)$ and total source current $I_{S}(t)$. Integrating this equation, gives the total increment in mobile charge which is represented by the area between these two curves. The dotted line shown in Figure 2.6(c) is labeled as $I_{T}(t)$, but this can be misleading if it is interpreted as varying with time, since $I_{T}$ is defined by steady-state conditions ${ }^{6}$; the dotted line represents a hypothetical or fictitious current assuming $d c$-like conditions applied at all times; i.e. $I_{D}(t)=-I_{S}(t)=I_{T}(\dot{\tau})$. Thus, by viewing the total source and drain currents simultaneously and with the aid of this $d c$-like transport current the charging current components of the source and drain $i_{S}$ and $i_{D}$, represent the amount that $I_{S}(t)$ and $I_{D}(t)$ differ from their respective transport current components. Thus,

$$
\begin{gathered}
I_{S}(t)=I_{T}+i_{S} \\
I_{D}(t)=-I_{T}+i_{D}
\end{gathered}
$$

Similarly, if these charging currents were to be defined as

$$
\begin{gathered}
i_{S}=\frac{d Q_{M S}}{d t} \\
i_{D}=\frac{d Q_{M D}}{d t},
\end{gathered}
$$

$Q_{M S}$ and $Q_{M D}$, though fictitious charges, associated with the source and drain respectively, are best thought of as being equal to the integral of the difference

6 According to (2.13) the total current going into any node may be broken up in to its dc and time-dependent components for the purpose of modelling. 
between their respective actual curnent curves (solid lines) and the $d c$ - like transport component (dotted lines); that is, in Figure 2.6(c), $Q_{M S}$ is given by the area between $-I_{S}(t)$ and $I_{T}$, and $Q_{M D}$ by the area between $I_{T}$ and $I_{D}(t)$.

From equations (2.24) $-(2.28)$ it follows that

$$
\frac{d Q_{M}}{d t}=\frac{d Q_{M S}}{d t}+\frac{d Q_{M D}}{d t}
$$

and therefore

$$
Q_{M}=Q_{M S}+Q_{M D}
$$

It is necessary therefore to find $Q_{M S}$ and $Q_{M D}$ in order to solve for $i_{S}$ and $i_{D}$ (and hence, $I_{S}(t)$ and $I_{D}(t)$ ), however (2.30) does not paint the true picture about $Q_{M S}$ and $Q_{M D}$ because it appears to be suggesting that they can be physically interpreted as separate components of the channel charge $Q_{M}$ that come from the source and drain respectively. This implies that $Q_{M}$ can be viewed as stored charge, but in fact, $Q_{M}$ is the measure of the net number of mobile carriers that can be found in the intrinsic device at any particular time. Furthermore, it makes no physical sense to think of $Q_{M S}$ and $Q_{M D}$ as coming from the source and drain, because all the mobile carriers enter the channel from the source and exit via the drain. Rather, these charges must be regarded as being associated with the source or drain boundary respectively, which are able to communicate with them; in this sense they constitute a two-lump representation of the distributed stored charge $Q_{M}$.

In order to solve for $i_{S}$ and $i_{D}, Q_{M}$ must be partitioned into its source and drain derived components, $Q_{M S}$ and $Q_{M D}$ respectively. Any partitioning methor may be used to serve this purpose. Four such methods for partitioning the mobile charge in the channel are discussed below. 


\subsubsection{Methods for Partitioning the Mobile Channel Charge $Q_{M}$}

Dutton et al. [9] : The partitioning of $Q_{M}$ into the charges $Q_{M S}$ and $Q_{M D}$ associated with the source and drain respectively, is made at some physical point along the channel $y_{o}$; therefore,

$$
\begin{gathered}
Q_{M S}=\int_{0}^{y_{0}} \frac{d Q_{m}}{d y} d y \\
Q_{M D}=\int_{y_{0}}^{L} \frac{d Q_{m}}{d y} d y
\end{gathered}
$$

The authors proposed to define $y_{0}$ as being the point along the channel where the surface potential is some constant fraction of the drain to source voltzge $V_{D S}$, where the transient current in the channel is zero. Hence, this point will vary for differing combinations of terminal voltages, since the surface potential distribution changes; $y_{o}$ is bias dependent and although this method would yield the exact source and drain terminal currents, it is very difficult to determine analytically.

For the sake of simplicity however, the authors chose to split $Q_{M}$ in half, assigning half the channel charge to $Q_{M S}$ and half to $Q_{M D}$, in both the linear and saturation regions. This method, though physically crude in its estimate, is suffciently accurate for most practical purposes and has the advantage of being very simple for use in most practical modelling applications. It is the basis of modelling $Q_{M}$ in SPICE'.

A. R. Boothroyd et al. [10] : The mobile charge is defined by dividing the channel into two equal halves, giving

\footnotetext{
7 SPICE is an analog circuit simulation software package, developed at the Electronics Research Laboratory of the University of California, Berkeley.
} 


$$
\begin{aligned}
& Q_{M S}=\int_{0}^{\frac{L}{2}} Q_{m} d y \\
& Q_{M D}=\int_{\frac{L}{2}}^{L} Q_{m} d y
\end{aligned}
$$

where $Q_{m}$ is the quasi static mobile charge density (per unit area) along the channel. The mobile channel charge is thus represented by a symmetrical two-lump model.

Chatterjee et al. [11] : $Q_{M S}$ and $Q_{M D}$ are obtained using a weighted method of partitioning the channel charge $Q_{M}$; the quantities and ratios of $Q_{M S}$ and $Q_{M D}$ are bias dependent. The cotal channel charge is determined first from the given charge distribution along the channel using steady state conditions for every region of operation. In saturation it is assumed that $Q_{M D}$ is zero, because the channel is inolated from the drain, therefore $Q_{M S}=Q_{M}$. In the linear region, $Q_{M}$ is split up according to certain boundary conditions which maintain continuity of $Q_{M S}$ and $Q_{M D}$, from the saturation region throughout the linear region. In the linear region, $Q_{M}$ is partitioned into:

$$
\begin{aligned}
& Q_{M S}=-W L C_{o x}\left(\frac{\left(V_{G S}-V_{T H}\right)}{2}+\frac{\alpha_{x} V_{D S}}{4}-\frac{\alpha_{x}^{2} V_{D S}^{2}}{24\left(V_{G S}-V_{T H}-\frac{\left.\alpha_{x} V_{D S}\right)}{2}\right)}\right. \\
& Q_{M D}=-W L C_{o x}\left(\frac{\left(V_{G S}-V_{T H}\right)}{2}-\frac{3}{4} \alpha_{x} V_{D S}+\frac{\alpha_{x}^{2} V_{D S}^{2}}{8\left(V_{G S}-V_{T H}-\frac{\left.\alpha_{x} V_{D S}\right)}{2}\right)}\right.
\end{aligned}
$$

where $\alpha_{x}=\alpha+\beta\left(V_{G S}-V_{T H}\right), \alpha$ and $\beta$ are short channel parameters which compensate for short channel effects.

Ward $[12,15]$ : Another weighted method for evaluating $Q_{M S}$ and $Q_{M D}$ is given by

$$
Q_{M S}=\int_{0}^{L}\left(1-\frac{y}{L}\right) Q_{m}(y) d y
$$




$$
Q_{M D}=\int_{0}^{L} \frac{y}{L} Q_{m}(y) d y
$$

for the mobile charge density distribution defined by

$$
Q_{m}\left(V_{g}, V_{y}\right)=-C_{o x}\left(V_{g}-V_{y}-\gamma \sqrt{V}_{y}\right)
$$

where all other necessary quantities are obtained using one-dimensional dc analysis; i.e.

$$
\begin{gathered}
d y=\frac{L}{I}\left(V_{g}-V_{y}-\gamma \sqrt{V_{y}}\right) d V_{y} \\
\frac{y}{L}=\frac{V_{g}\left(V_{y}-V_{s}\right)-\frac{2}{3} \gamma\left(V_{y}^{\frac{3}{2}}-V_{s}^{\frac{3}{2}}\right)-\frac{1}{2}\left(V_{y}^{2}-V_{s}^{2}\right)}{I} \\
I=V_{g}\left(V_{d}-V_{s}\right)-\frac{2}{3} \gamma\left(V_{d}^{\frac{3}{2}}-V_{s}^{\frac{3}{2}}\right)-\frac{1}{2}\left(V_{d}^{2}-V_{s}^{2}\right)
\end{gathered}
$$

and where,

$$
\begin{gathered}
V_{g}=V_{G S}-V_{B S}-V_{F B} \\
V_{d}=V_{D S}-V_{B S}+2 \psi_{F} \\
V_{d}=V_{S B}-V_{B S}+2 \psi_{F} \\
V_{y}=V(y)-V_{B S}+2 \psi_{F} \\
\gamma=\frac{\sqrt{2 \epsilon_{s i} q N_{A}}}{C_{o x}}
\end{gathered}
$$

and $\psi^{\prime} F$ is the Fermi voltage. Unlike Chatterjee's method this is a weighted method according to the distance along the channel, not only bias; thus, $Q_{M S}$ is progressively reduced to zero as the drain is approached, according to the distribution of channel charge $Q_{m}\left(V_{g}, l_{y}\right)$. Likewise, the influence of the drain on the associated charge $Q_{M D}$, is finally reduced to zero at the source, while the rate of reduction 
along the channel is dictated by the distribution of the total mobile charge $Q_{M}$. This method also permits the existence of a drain-associated charge $Q_{M D}$ while the device is operating in saturation, hence, is better able to predict capacitances like $C_{D G}$ which are actually non-zero in saturation.

Though this method of evaluating $Q_{M S}$ and $Q_{M D}$ is mathematically sound, it is very complex for purposes of circuit simulation. An approximation of the results obtained using this method is given by

$$
\begin{aligned}
& Q_{M S} \simeq \frac{3}{5} Q_{M S a t}+\frac{3}{10}\left(Q_{M}-Q_{M S a t}\right) \\
& Q_{M D} \simeq \frac{2}{5} Q_{M S a t}+\frac{7}{10}\left(Q_{M}-Q_{M S a t}\right)
\end{aligned}
$$

where $Q_{M S a t}$ is the channel charge $Q_{M}$ in saturation, approximated by

$$
Q_{M S a t} \simeq-\frac{2}{5} C_{o x}\left(V_{g}-V_{s}-\gamma \sqrt{V_{y}}\right)
$$

this being better suited for simulation purposes. Quite good agreement with measured capacitances has been reported by Ward for a model derived on this basis using the same approximations as Meyer in the representation of $Q_{G}, Q_{B}$.

\subsection{Short Channel MOS Transistor Capacitances}

It has been emphasized throughout this chapter that a proper treatment of both the numerical methods and the device phvsics is necessary to accurately predict the transient behavior of the device for circuit simulation purposes. So far, the discussion has been concerned with determining the dynamic or ac characteristics of long channel MOSFETs. Whereas the dc characteristics of small geometry devices have been well understood and modelled for many years[1-5], only recently has attention been given to investigating their dynamic characteristics[6-21]. As the density and speed requirements of VLSI circuits are always iacreasing, so too has 
the demand to understand and properly model the transient behavior of short channel devices, implying of course, an accurate derivation of the capacitances of such small geometry devices.

Due to their first order importance in transient analysis, the gate capacitances of short channel devices were the first to be investigated[9,16-21]. For the remainder of this thesis, the emphasis will be on these capacitances, namely $C_{G D}, C_{G S}$, and $C_{G B}$.

Short channel gate capacitances were first studied and analyzed in detail by R. W. Dutton et al. at Stanford University, who have presented results obtained from both measurement $[16,17,20]$ and two-dimensional numerical simulations using PISCES-II[16]. The emphasis was to observe extreme short channel effects on the gate capacitances for the purposes of better analyzing the mechanisms responsible, rather than studying such effects on more practical devices. The following process parameters were used: bulk resistivity $\rho=20 \Omega-c m$, oxide thickness $T_{o x}=730 \AA$, junction depth $x_{j}=0.4 \mu m$, with a peak concentration of the arsenic profile in the source and drain of $9 \times 10^{19} \mathrm{~cm}^{-3}$ and a Gaussian implanted channel boron profile with a dosage of $2.8 \times 10^{11} \mathrm{~cm}^{-2}$. The effective channel width $W_{\text {eff }}=99.2 \mu \mathrm{m}$ and the effective channel lengths varied between $L_{e f f}=7.8 \mu \mathrm{m}$ (long channel case) ${ }^{8}$, to $L_{\text {eff }}=0.8 \mu \mathrm{m}$.

The gate capacitances obtained from measurement shown in Figure 2.79, show a noticealle shift to the left of the capacitance characteristics and an increased spreading out or rounding off of $C_{G S}$ and $C_{G D}$, thereby making the transition between the saturation and triode regions more vague as the effective channel length is reduced. It should also be noticed from the normalized gate capacitance characteristics shown in Figure 2.7(b), that the extrinsic capacitance component becomes

${ }^{8}$ Given these process parameters, the device with $L_{e f f}=7.8 \mu \mathrm{m}$ already exhibits considerable short channel effects, as can be seen by the rounded off capacitance characteristics shown in Figure 2.7 and Figure 2.8. However, for comparison purposes, it serves well to compare with the extreme short channel effects of the device with $L_{e f f}=0.8 \mu m$.

9 Corresponds to Figure 1 of [16]. 
(a)

Cad,Cas, $C_{G o}(f F)$
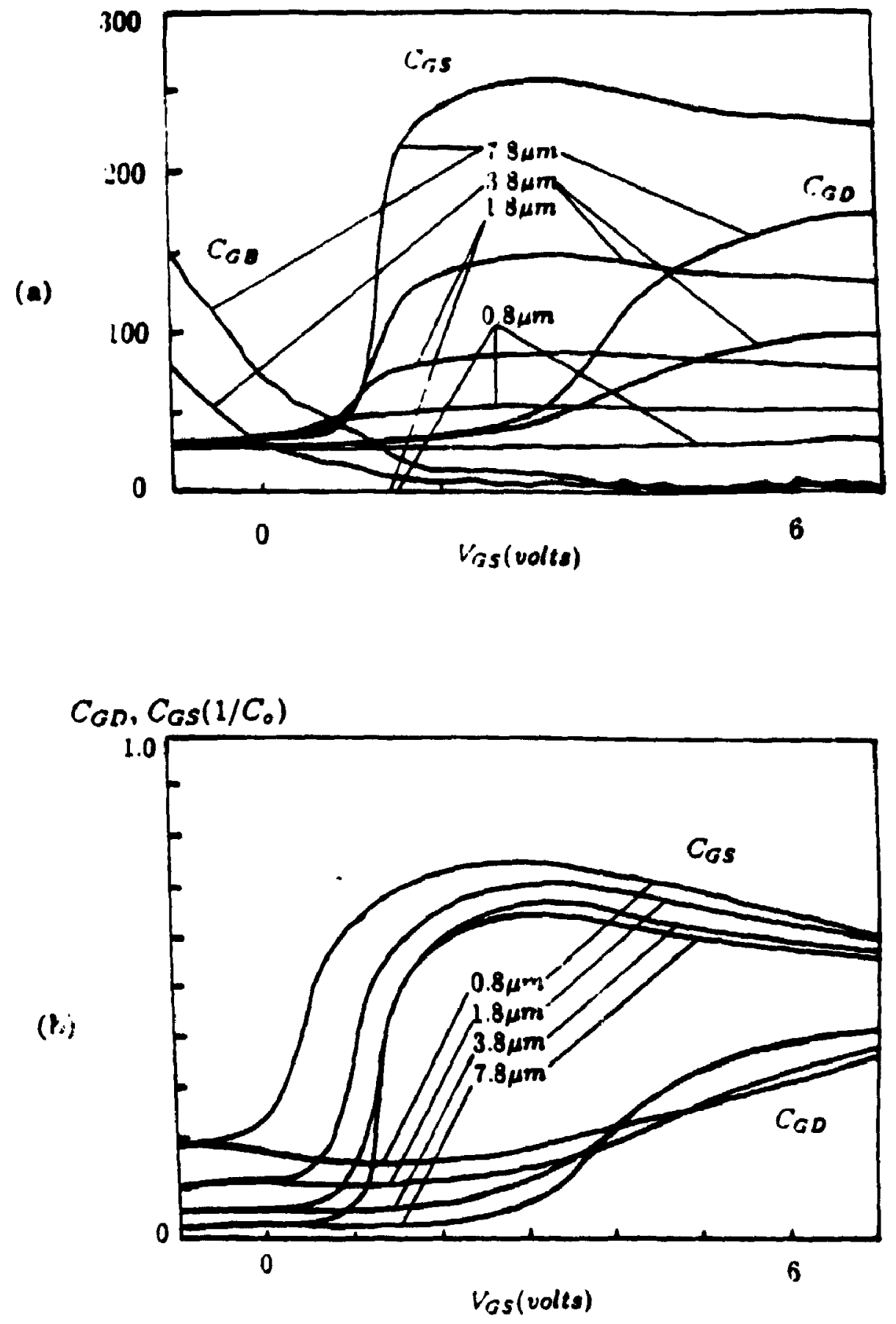

Figure 2.7 Capacitances obtained from measurement[16]; (a) $C_{G D}, C_{G S}, C_{G B}$ and (b) normalized $C_{G D}, C_{G S}$. 
more dominant, compared to the overall capacitance of the device as the dimensions of the device are reduced.

It was discovered that two-dimensional fringing effects near the source and drain, and velocity saturation are the two mechanisms mainly responsible for short channel effects in MOS transistor capacitances. A detailed analysis of the short channel gate capacitance characteristics are given below:

$C_{G D}:$ Starting from the accumulation region with reference to Figure $2.8(\mathrm{a})^{10}$ the intrinsic capacitance component of $C_{G D}$ is very small. This is so, because the heavy accumulation of holes under the gate shields off the electric field from the drain and hence, only a small inner fringing capacitance is able to exist in this region of operation. As the gate voltage is increased, the number of holes (and therefore the amount of shielding) is reduced, thereby indicated by the gradual increase in $C_{G D}$.

Depletion in the short channel device occurs almost instantaneously at some relatively low gate voltage, because the potential in the entire channel region is strongly affected by the adjacent source and drain, which are at a higher bias point than the gate, thereby depleting the area under the gate completely. In the long channel device there is a gradual depletion of holes, this being almost entirely controlled by the gate. As a consequence, $C_{G D}$ of the shorter device, consisting of the small inner fringing capacitance component, remains fairly constant right up until threshold is reached, whereas $C_{G D}$ of the long channel device increases with increasing $V_{G S}$ since the amount of shielding of the inner fringing capacitance is gradually reduced. It must be born in mind however, that for the long channel device studied by Dutton et al., the magnitude of the inner fringing capacitance coupling is significant throughout the length of the channel, and is not local to the drain region alone.

At around threshold, there is a noticeable dip in $C_{G D}$. This is due to the

${ }^{10}$ Corresponds to Figure $6(a, b)$ of $[16]$. 
$C_{G D}, C_{G s}, C_{G O}\left(1 / C_{0}\right)$

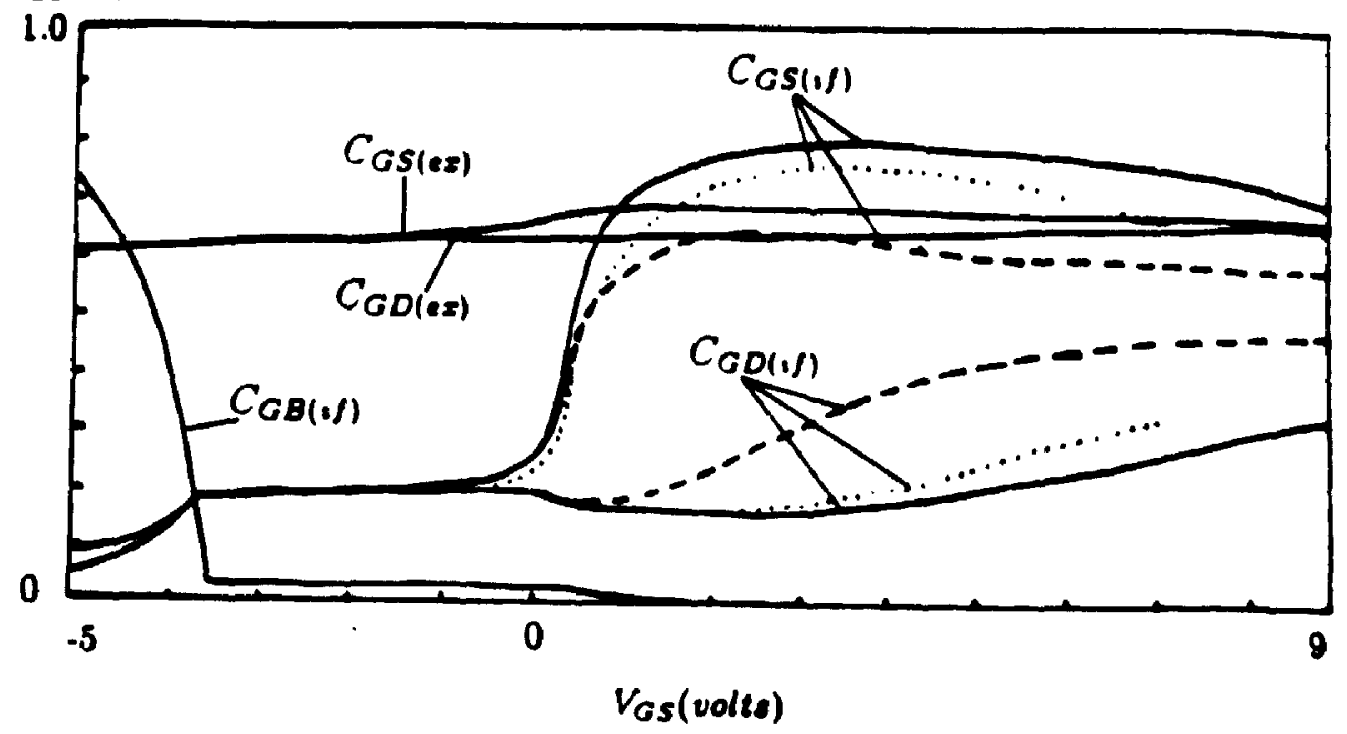

(a)

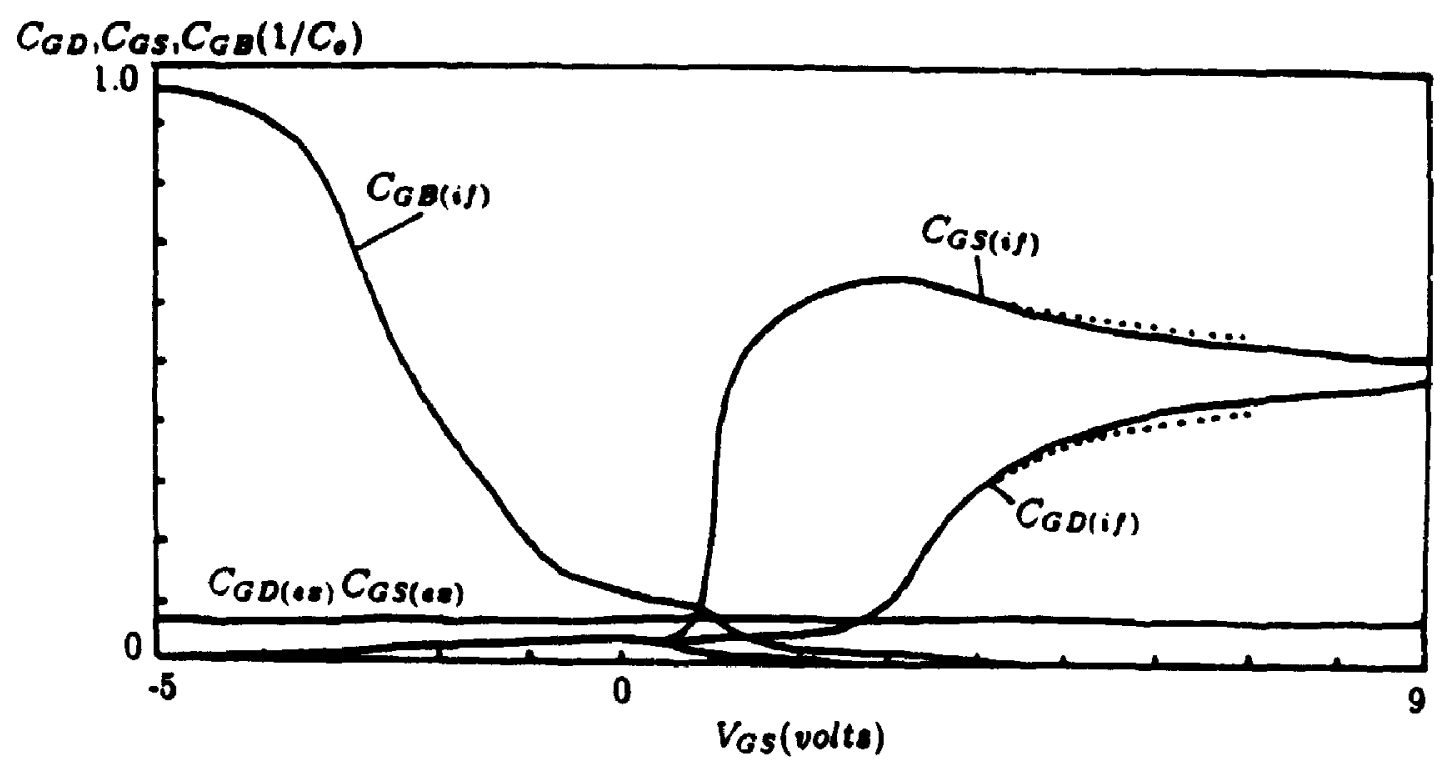

(b)

Figure 2.8 Normalized gate capacitances obtained from 2D simulation, with velocity sa iuration (solid), constant mobility (dashed) and measurement (dotted) [16]; $V_{B S}=-2.0 \mathrm{~V}, V_{D S}=2.0 \mathrm{~V}$; (a) $L_{\text {ess }}=0.8 \mu \mathrm{m}$, (b) $L_{\text {efs }}=$ $7.8 \mu \mathrm{m} . C_{G D(e x)}$ and $C_{G S(e x)}$ are extrinsic capacitance components due to outer fringing coupling effects. 
partial shielding of the inner fringing component of capacitance near the source, where the channel begins to form. As the channel is formed more strongly, $C_{G D}$ associated with the 2D field region near the drain, tends to decrease as more of the inner fringing component is being shielded, but at the same time, a more dominant influence of the gate potential on the surface potential near the drain tends to cause $C_{G D}$ to increase with increasing gate voltage. In the short channel device the entire channel region is involved in the fringing area of the drain; hence, the entire potential distribution of the channel will experience a change given a small change in the drain voltage $V_{D S}$, and the device goes into the linear region, where essentially all of $C_{G D}$ is made up of the gate-to-channel capacitance, in a smooth gradual transition.

It was also discovered by Dutton et al. that velocity saturation effects are partly responsible for suppressing the saturation pinch-off-point, thus spreading out the characteristics further and making the transition between the saturation and linear regions even more vague. Figure 2.8 shows that for simulations where the mobility $\mu$ is kept constant, the characteristics behave more like those of a long channel device. The effect of velocity saturation is best explained by looking at the $\Delta \psi_{s}$-distributions ${ }^{11}$ shown in Figure $2.9^{12}$. Velocity saturation occurs near the drain edge because of increased scattering effects due to the high fields in that region causing local reduction in mobility. This in turn serves to increase the local resistivity of the channel around the drain. It follows therefore that there would be a greater drop in the potential near the drain region, compared to the case with constant $\mu$. Since the potentials at the source and drain are fixed, the area under

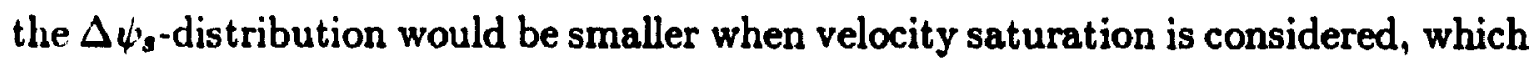
is proportional to $\Delta Q_{G}$. It follows therefore that $C_{G D}$ is reduced, resulting in lower and more spread-out characteristics. The $\Delta \psi_{s}$-distribution of the short channel

II A point on $\Delta \psi_{s} \sim V_{G S}$ distribution corresponds to the difference in surface potential between two consecutive simulations, with a difference in the drain voltage, $\Delta V_{D S}=0.01$ volts.

12 Corresponds to Figure 6 of [16]. 
device while in the linear region of operation looks more like that in saturation, mainly because of the velocity saturation effects. As shown in Figure 2.8, using constant mobility, $C_{G D}$ rises considerably as it would in the long channel case, since the dominant capacitance component is the gate-to-channel capacitance, which is much larger than the inner fringing component. Huwever, as shown by the figure, the magnitude of $C_{G D}$ is reduced considerably when velocity saturation effects are considered.

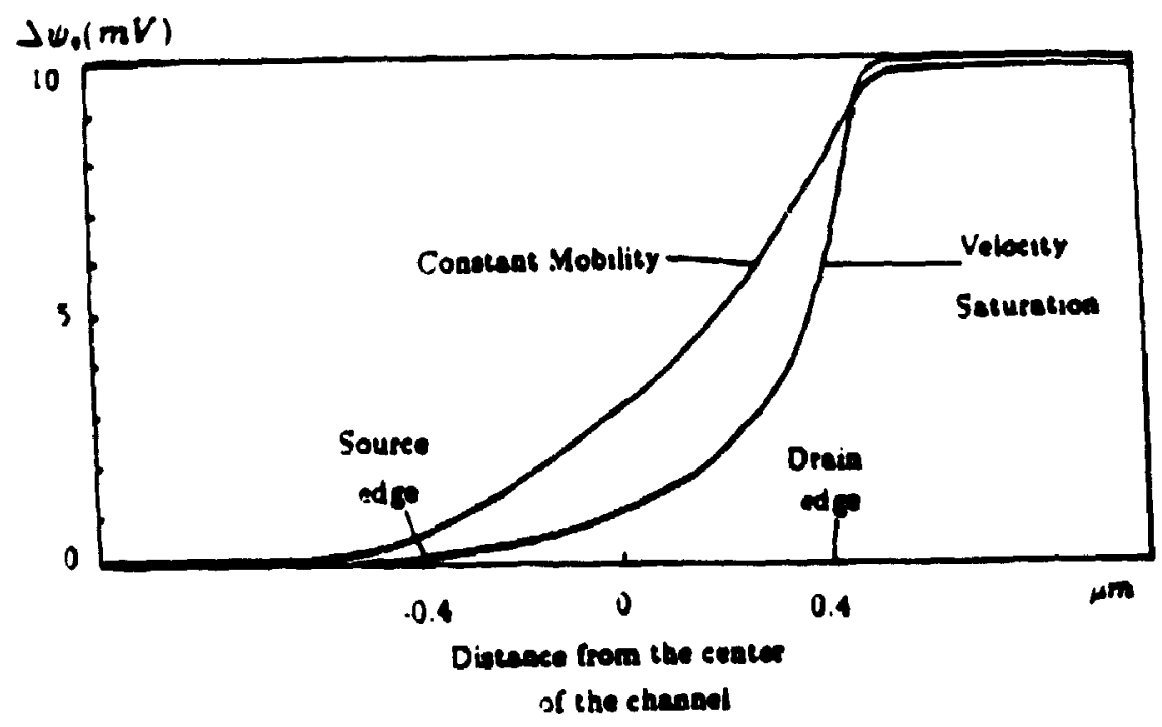

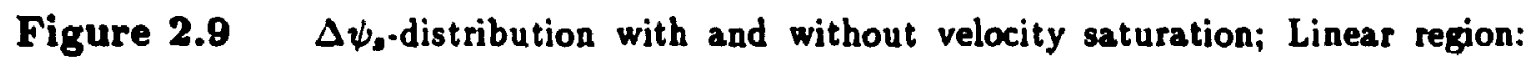
$V_{D S}$ changes from $2.00 \mathrm{~V}$ to $2.01 \mathrm{~V}$. $V_{G S}=4.0 \mathrm{~V}, V_{S B}=2 \mathrm{~V} ; L_{e f f}=$ $0.8 \mu m$.

$C_{G S}$ : The behavior of $C_{G S}$ is similar to that of $C_{G D}$ right up to threshold and for essentially the same reasons. When threshold is reached, the channel becomes inverted near the source, thus shielding the small inner fringing component, while at the same time forming a new, more dominant gate-to-channel capacitance, thereby increasing $C_{G S}$. The characteristics however retain a rounded off appearance due to the influence of the two-dimensional effects at the edges of the channel.

In the linear region of operation $C_{G B}$ is essentially zero since the surface charge under the gate effectively shields off the electric field from reaching the bulk, hence, 
the total gate capacitance is made up of $C_{G D}$ and $C_{G S}$. Since, $C_{G D}$ is reduced by the velocity saturation effects in this region, $C_{G S}$ must likewise be increased by the same amount in order to make up the total gate capacitance (since $C_{G B}$ is essentially zero when the channel is under strong inversion. The difference in $C_{G S}$, with and without considering velocity saturation is shown in Figure 2.8.

$C_{G B}:$ As mentioned above in the discussion of $C_{G D}$, the short channel device enters depletion suddenly and at a lower gate bias than the long channel device, because the fringing effects of the source and drain help significantly in removing the holes from the channel. Therefore, leaving this condition as the gate potential is raised above threshold, there is a sudden reduction in $C_{G B}$, from the large gateto-substrate capacitance component of depletion operation down to the small inner fringing component when the channel is formed. This is further reduced to almost nothing as the device enters the linear region and the inversion layer charge shields off the electric field from the bulk.

It has become clear from the above analysis that the small inner fringing capacitances become a significant part of the intrinsic capacitances of the short channel device and cannot be neglected as in the long channel case. It must also be pointed out that the external capacitance component of the short channel device is independent of the channel length and of the applied voltages, as can be observed in Figure 2.8 and therefore assumes a greater proportion of the total device capacitance or similarly, the intrinsic capacitance component of the short channel device is responsible for a smaller part of the total capacitive load of the device in the circuit. 


\section{Chapter 3 \\ Extraction of Gate Capacitances from MINIMOS Simulation}

\subsection{Introduction}

With the emergence of VLSI many demands have arisen to understand and model both the dc and ac characteristics of short channel devices, yet the attention that these two areas have received has been far from equal. Whereas the static behavior of short channel MOSFETs has been well understood for several years now, the investigation of their transient characteristics has begun only recently, as discussed in the previous chapter.

When this project began, no work had been done on the determination of the capacitance characteristics of short channel MOSFETs from device simulation. Knowledge of their properties had been gained from experimental measurements taken by Dutton et al.[16] and Ko et al.[21]. There was a large interest therefore in obtaining such characteristics using $2 \mathrm{D}$ device simulation, in order to study their properties and guide the derivation of efficient and simple analytical models that could be used for the purposes of circuit simulation.

Since then, short channel gate capacitances have been examined by Dutton et al. at Stanford, using PISCES-II[22], a 2D numerical device simulator, as was discussed in Section 2.4. That work was concerned with the study of device struccures whose substrate doping was very low, such that extremely pronounced short char zel effects could be observed, providing a clear indication of the mechanisms responsible for the changes in the characteristics subject to such effects.

This chapter will deal with the process of extracting gate capacitances of lory and short channel MOSFETs using the numerical MOSFET simulator MINIMOS, 
and will consider device structures with more practical processing parameters than those used by Dutton.

MINIMOS is a 2D numerical simulation package for the planar MOS transistor, which solves the 2D Poisson and current continuity equations using the method of finite differences. It normally generates the mesh pattern automatically ${ }^{1}$ based on the given input bias conditions and can provide solutions in the silicon as well as inside the oxide. The physical quantities that are available at the output include doping profile, electric potential, transverse and lateral electric fields, space charge density, quisi-Fermi levels, carrier concentration, lateral and transverse current densities, mobility distribution and avalanche generation rates.

The processing parumeters that were chosen for the devices were based on Northern Telecom's (NT) 3 micron process. Typical fabricated transistors have a gate overlap of 0.35 microns $(\mu m)$ on each side of the channel, thus making the effective channel length of the device $0.7 \mu \mathrm{m}$ smaller than the specified gate length. The desired profile and the effective channel lengths of the long (LD), medium (MD) and short (SD) channel devices of $49.3,2.3$ and $1.0 \mu \mathrm{m}$ respectively, were obtained by trial and error MINIMOS simulations, varying the time and temperature until the required processing parameters were met; these are:

$$
\begin{gathered}
N_{A}=1.4 \times 10^{16} \mathrm{~cm}^{-3} \\
T_{o x}=500 \AA \\
x_{j}=0.6 \mu \mathrm{m} \\
C_{o x}=6.9 \times 10^{-8} \frac{\mathrm{F}}{\mathrm{cm}^{2}} \\
Q_{s s}=-6.8 \times 10^{-9} \frac{\text { Coul }}{\mathrm{cm}^{2}} \quad \text { (effective value) }
\end{gathered}
$$

1 This relieves the user of the task of setting up the grid, but deprives him of its control. l'nder certain circumstances, to be discussed later, it is possible tn overide changes of grid configuration between simulations. 
It should be noted that both the medium and short channel devices, as designated here, exhibit short channel effects, as will be shown later. These names were chosen simply for purposes of differentiating the three channel lengths and not as an accurate representation of physical size.

\subsection{Feasibility of MINIMOS for Investigating Capacitance Characteristics}

Before undertaking the study of short channel gate capacitances by means of numerical simulation, it was necessnry first to establish the feasibility of MINIMOS for this purpose.

The process of evaluating the gate capacitances $C_{G D}, C_{G S}, C_{G B}$ using MINIMOS is basically one of taking the difference between values of gate charge, obtained by integrating charge density along the gate, for two separate static numerical simulations correspondirg to incrementally different values of bias voltage conditions. This is a somewhat complex numerical procedure, and before placing reliance on the results obtained by this means it was necessary first to establish the simulation accuracy of MINIMOS in this context and then verify the feasibility of extracting capacitance characteristics by differencing successive simulations. To satisfy the requirement of simulation accurac $y^{\prime}$ it was required that device characteristics derived from simulation be reconciled with the known characteristics given by existing long channel MOSFET theory. When this part of the investigation for large geometry devices proved satisfactory, it would be possible to examine the fcasibility of actually evaluating gate capacitances from MINIMOS.

\subsubsection{Surface Potential Distribution $\psi_{s}(y)$}

The simulation accuracy of MINIMOS was investigated by comparing the surface potential distributions $\psi_{s}(y)$ from simulation with those from established long channel theory.

Long channel theory (LCT)[3] calculations were made to determine the bias 
conditions for the $49.3 \mu \mathrm{m}$ device in the saturation and triode regions. Based on the given biases $V_{S B}=0 \mathrm{~V}$ and $V_{D S}=2.0 \mathrm{~V}$, the saturation point was found to be $V_{\text {gsat }}=3.54 \mathrm{~V}$ and hence the selected bias points for the device operating slightly into the saturation region and triode region were $V_{G S}=3.35 \mathrm{~V}$ and $V_{G S}=3.75 \mathrm{~V}$, respectively. The desired profile and the effective channel length (here deïued, as between metallurgical junction points along the silicon surface) for the long channel device had to be determined through trial and error by varying the input values of time and temperature in the PROFILE IDENT as well as the gate length $L_{g}$, which is what is actually specified in the MINIMOS input, until the correct value of junction depth $\left(x_{j}=0.6 \mu \mathrm{m}\right)$ and effective channel length $\left(L_{\text {eff }}=49.3 \mu \mathrm{m}\right)$ were obtained. The appropriate values of time and temperature that were found to give the correct junction depth were 6500 minutes and $1000^{\circ} \mathrm{C}$ respectively. This profile also produced a subdiffusion of $0.5319 \mu \mathrm{m}$ underneath the gate and hence the gate length that had to be specified at the input was $L_{g}=2(0.5319 \mu \mathrm{m})+49.3 \mu \mathrm{m}=$ $50.3638 \mu \mathrm{m}$ in order to get the correct effective channel length.

Figure 3.1 shows the surface potential distributions obtained from MINIMOS and long channel theory in both regions of operations. For the above operating conditions the $\psi_{s}$-distribution from long channel theory had to be shifted by an amount equal to the subdiffusion distance given by MINIMOS $(0.5319 \mu \mathrm{m})$ in order to align the source and drain edges as close as possible with those from simulation. It must be noted however, that the alignment with the simulated distributions is approximate, since it is not possible to know exactly where the source and drain edges of the device are located judging from the MINIMOS output, these being subject to the change in the doping profile and not some clearly defined boundary. In the strict sense therefore, the actual effective channel length must be approximate and cannot be clearly defined as in the case of long channel theory. Any discrepancies in the alignment however, are certainly not noticeable in the long channel case (Figure 3.1) and good agreement is observed for both the saturation and triode regions of operation. 


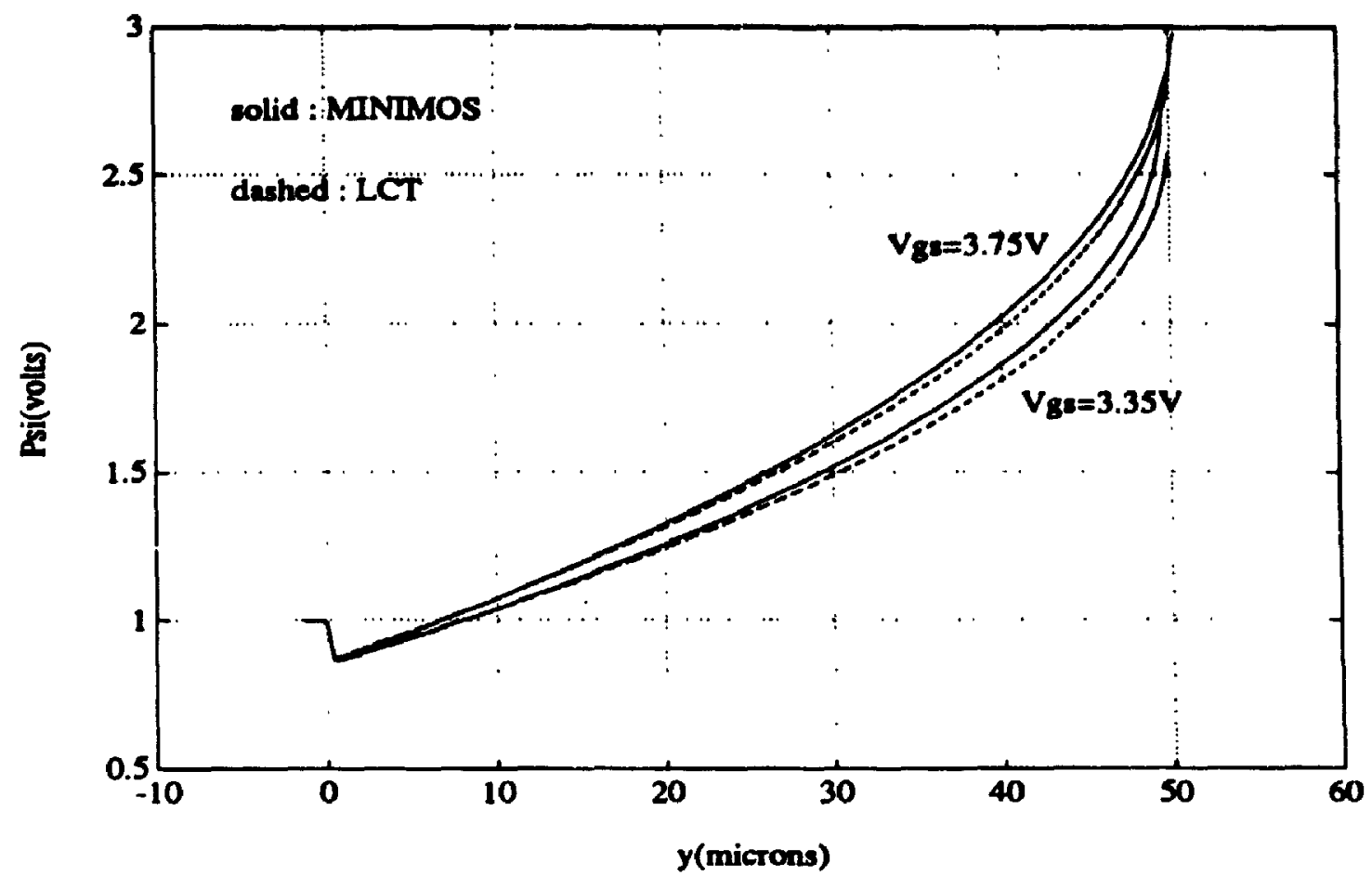

Figure 3.1 $\quad \psi_{s}(y)$ from MINIMOS and long channel theory for saturation $\left(V_{G S}=\right.$ $3.35 \mathrm{~V})$ and triode $\left(V_{G S}=3.75 \mathrm{~V}\right)$ regions of operation; $V_{D S}=2.0 \mathrm{~V}$, $V_{S B}=0 \mathrm{~V} ; L_{\text {eff }}=49.3 \mu \mathrm{m}$.

Long channel theory seems to predict the same distribution for $\psi_{\mathbf{s}}(y)$ near the source end of the device but begins to drop below that from MINIMOS as the drain is approached; this is because the $1 D$ analysis does not consider the 2D effects present near the drain or the changes in the mobility distribution $\mu(y)$, which effectively serve to raise the $\psi_{s}$-distribution near the drain edge of the channel. The decrease in the $\psi_{s}$-distributions predicted by long channel theory are expected to overestimate the gate charge $Q_{G}$ compared to MINIMOS; this is indeed the case, as will be seen in the following section, and Table 3.1.1.

A marked increase is expected in the difference between the $\psi_{s}$-distributions of the smaller $2.3 \mu \mathrm{m}$ device, due to the increased influence of the 2D edge effects, which raise $\psi_{s}(y)$ even further; this is indeed observed to be the case for both regions of operations, as shown in Figure 3.2. The gate voltages were chosen as 
$V_{G S}=3.0 \mathrm{~V}$ (saturation) and $V_{G S}=3.5 \mathrm{~V}$ (triode), which take into account the change of the threshold voltage point due to the influence of $\mathrm{SCE}^{2}$.

These curves illustrate the influence of short channel effects on the $\psi_{\mathrm{s}}$-distributions, showing considerable differences between the results of simulation and long channel theory. MINIMOS, being a 2D numerical device simulator, is able to account for the edge effects near the source and drain as well as the change in mobility of the electrons near the drain, whereas long channel theory, being a 1D analysis assumes constant mobility ${ }^{3}$ along the total length of the channel. The edge effects of the shorter device therefore, tend to push the surface potential distribution to a higher value, than would have been the case, had these effects been negligible; this can be observed in Figure 3.2.

\subsubsection{Calculating the Gate Charge $Q_{G}$}

In order to extract gate capacitance characteristics foun MINIMOS, a relationship must be formed between the gate charge $Q_{G}$ and the terminal voltages. There are various methods of evaluating $Q_{G}$ from simulation which will be discussed here, but in all cases these involve spacially dependent quantities such as potential or electric field.

The feasibility of evaluating $Q_{G}$ is examined for a long channel MOSFET by: 1) investigating the accuracy of the physical properties such as surface potential distribution $\psi, s(y)$ and the transverse (i.e. normal to the surface) electric field distributions $\mathcal{E}_{s i}(y)$ in the silicon (just underneath the $\mathrm{SiO}_{2} / \mathrm{Si}$ interface) and $\mathcal{E}_{o x}(y)$

2 The shift in the threshold voltage due to short channel effects was calculated using a trapezoidal approximation[25], which is based on the profile of the potencial distribution in the bulk of the intrinsic MOSFET given by MINIMOS for the case of the $2.3 \mu \mathrm{m}$ device, operating just above threshold (i.e. $V_{G S}=1.0 \mathrm{~V}$ ). The calculated estimate of the shift is $\Delta I_{T H} \simeq-0.30 \mathrm{~V}$. thus moving the threshold point of the $2.3 \mu \mathrm{m}$ device to $V_{T H} \simeq 0.45 \mathrm{~V}$.

3 The value of mobility used for the long channel calculations was $\mu_{0}=1200\left(\mathrm{~cm}^{2} / \mathrm{V} \cdot \mathrm{sec}\right)$ Sze[23], pp. 29. 


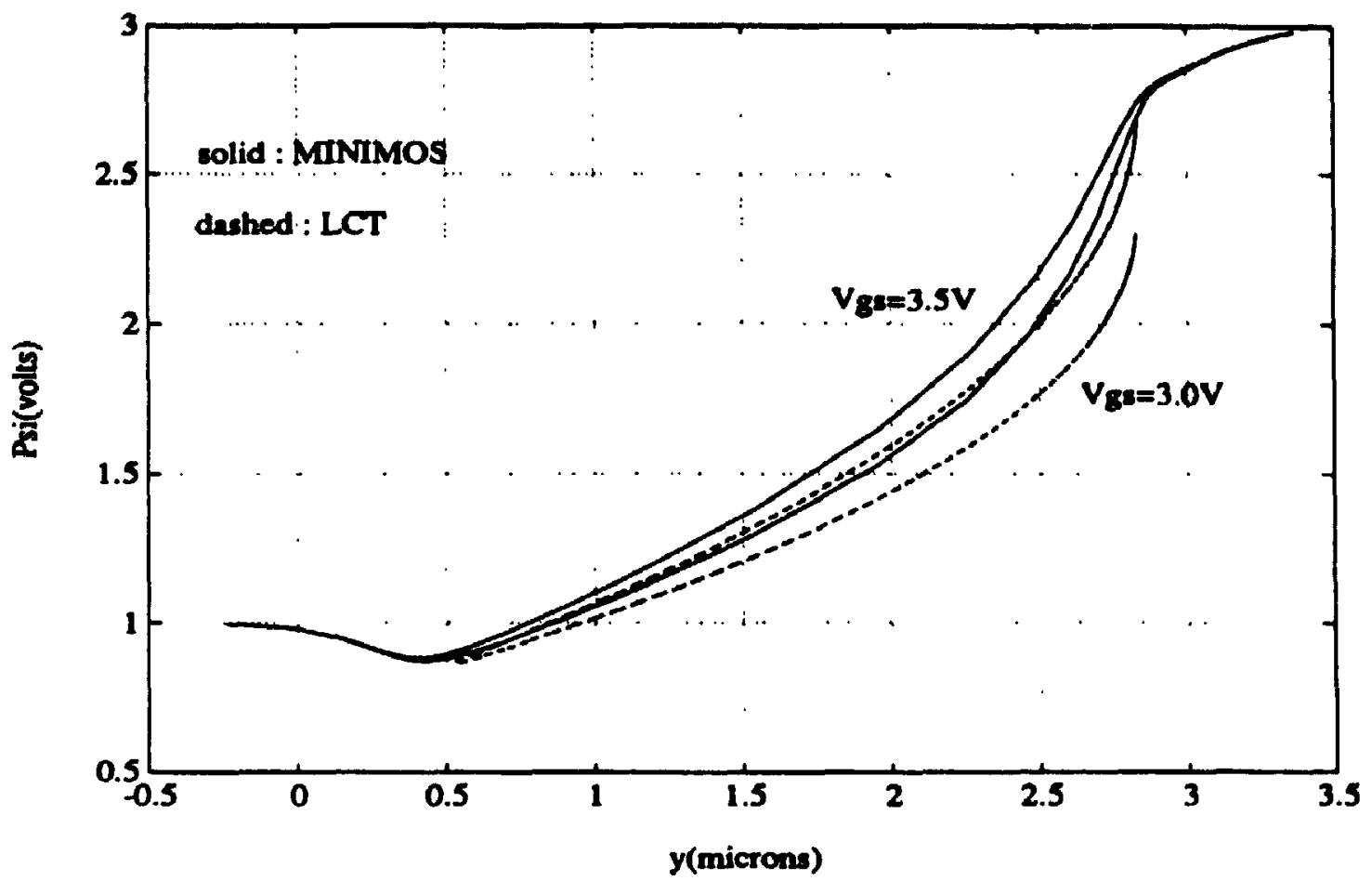

Figure 3.2 $\psi_{s}(y)$ from MINIMOS and long channel theory for saturation ( $V_{G S}=3.0 \mathrm{~V}$ ) and triode $\left(V_{G S}=3.5 \mathrm{~V}\right)$ regions of operation; $V_{D S}=2.0 \mathrm{~V}, V_{S B}=0 \mathrm{~V}$; $L_{e f f}=2.3 \mu m$.

inside the oxide; 2 ) comparing the results of $Q_{G}$ obtained by integrating the various output quantities from MINIMOS with those from LCT.

Assuming 1D conditions, the total gate charge may be calculated as

$$
Q_{G}=C_{0 x} \int_{0}^{L}\left(V_{G B^{\prime}}-\psi_{s}(y)\right) d y
$$

or

$$
Q_{G}=\int_{0}^{L} \epsilon_{x} \mathcal{E}_{x}(y) d y
$$

where $V_{G B}{ }^{\prime}=V_{G B}-V_{F B}$, and $V_{F B}$ is the flatband voltage, $\psi_{s}(y)$ is the surface potential distribution along the total effective channel length and $\epsilon_{x}$ is the permittivity of the oxide or semiconductor depending on the medium in which the transverse electric field distribution $\mathcal{E}_{x}(y)$ is being considered. The numerical approximation 
of the integrals in (3.1) and (3.2) is obtained by applying Simpson's $\frac{1}{3}$ rule; that is (3.1) is evaluated as

$$
Q_{G} \simeq C_{o x}\left[\left.\left(V_{G B}-V_{F B}\right) y\right|_{0} ^{L}-\frac{\Delta y}{3}\left(\psi_{s 0}+4 \psi_{a 1}+2 \psi_{s 2}+4 \psi_{a 3}+\ldots+4 \psi_{s n-1}+\psi_{s n}\right)\right]
$$

where, $\Delta y=\frac{l}{n}$ and (3.2) is evaluated as

$$
Q_{G} \simeq \epsilon_{x}\left[\frac{\Delta y}{3}\left(\mathcal{E}_{0}+4 \mathcal{E}_{1}+2 \mathcal{E}_{2}+4 \mathcal{E}_{3}+\ldots+4 \mathcal{E}_{n-1}+\mathcal{E}_{n}\right)\right]
$$

$Q_{G}$ was evaluated using (3.4), from the transverse electric field distribution in the silicon, at the oxide interface $\mathcal{E}_{s i}(y)$, and inside the oxide $\mathcal{E}_{o x}(y)$, using the distribution at both the gate boundary and the silicon interface.

The gate charge was evaluated first for the long channel device (LD) operating in saturation and triode, just above and below the saturation point for the device, which was calculated from long channel theory (see Section 3.2.1). An investigation of the $2.3 \mu \mathrm{m}$ device (MD) was also done in order to observe the influence of the two-dimensional effects on the shorter device.

$Q_{G}$ was evaluated first by the application of (3.3), using the surface potential distribution $\psi_{s}(y)$, both from MINIMOS and from LCT, which have been investigated in the previous section and the results obtained were found to be in good agreement for large channel lengths (Table 3.1.1(a), (b)). The results of $Q_{G}$ for both the LD and MD devices operating in the saturation and triode regions are listed in Table 3.1.1 and 3.1.24. It should be noted that for both regions of operation the values of $Q_{G}$ that were calculated from LCT, though very close, are consistently larger than those obtained from simulation (see Table 3.1.1(a), (b)). This was expected, since MINIMOS is able to represent the small influence of the 2D edge effects even in the $49.3 \mu \mathrm{m}$ device, which are reflected in the $\psi_{s}$-distribution

\footnotetext{
4 is the number of points that were employed in the calculation of $Q_{G}$ using (3.3) and (3.4) from LCT and MINIMOS, and was chosen to be sufficiently large so that the accuracy of $Q_{G}$ was not affected.
} 


\begin{tabular}{|c|c|c|c|}
\hline & $\begin{array}{l}\text { Method used to } \\
\text { evaluate } Q_{G}\end{array}$ & $\begin{array}{c}Q_{G}(F \cdot V / \mathrm{cm}) \\
V_{G S}=3.35 V \\
\text { (Saturation) }\end{array}$ & $\begin{array}{c}Q_{G}(F \cdot V / \mathrm{cm}) \\
V_{G S}=3.75 V \\
\text { (Triode) }\end{array}$ \\
\hline $\mathbf{a}$ & $\begin{array}{c}\psi_{*}(\mathrm{LCT}) \\
\% \text { difference }\end{array}$ & $\begin{array}{c}9.26 \times 10^{-10} \\
+1.09\end{array}$ & $\begin{array}{c}1.027 \times 10^{-9} \\
+1.18\end{array}$ \\
\hline $\mathrm{b}$ & $\begin{array}{c}\psi_{s} \text { (MINIMOS) } \\
\% \text { difference }\end{array}$ & $\begin{array}{c}9.16 \times 10^{-10} \\
\end{array}$ & $1.015 \times 10^{-9}$ \\
\hline c & $\begin{array}{c}\mathcal{E}_{s i}(\text { MINIMOS) } \\
\quad \% \text { difference }\end{array}$ & $\begin{array}{c}6.895 \times 10^{-10} \\
-24.73\end{array}$ & $\begin{array}{c}7.621 \times 10^{-10} \\
-24.92\end{array}$ \\
\hline $\mathrm{d}$ & $\begin{array}{c}\mathcal{E}_{o x} \text { (MINIMOS) } \\
\% \text { difference }\end{array}$ & $\begin{array}{c}9.16 \times 10^{-10} \\
0\end{array}$ & $\begin{array}{c}1.0155 \times 10^{-9} \\
+0.05\end{array}$ \\
\hline
\end{tabular}

Table 3.1.1 Comparison of $Q_{G}$ determined by various methods. $L_{e f f}=49.3 \mu \mathrm{m}$; $V_{D S}=2.0 V, V_{S B}=0 V ;$ Differences taken with respect to $\psi_{0}($ MINIMOS $)$.

\begin{tabular}{|c|c|c|c|}
\hline & $\begin{array}{c}\text { Method used to } \\
\text { evaluate } Q_{G}\end{array}$ & $\begin{array}{c}Q_{G}(F \cdot V / \mathrm{cm}) \\
V_{G S}=3.0 \mathrm{~V} \\
\text { (Saturation) }\end{array}$ & $\begin{array}{c}Q_{G}(F \cdot V / \mathrm{cm}) \\
V_{G S}=3.5 \mathrm{~V} \\
\text { (Triode) }\end{array}$ \\
\hline $\mathrm{e}$ & $\begin{array}{c}\psi_{\mathrm{s}}(\mathrm{LCT}) \\
\% \text { difference }\end{array}$ & $\begin{array}{c}3.90 \times 10^{-11} \\
+5.14\end{array}$ & $\begin{array}{c}4.49 \times 10^{-11} \\
+3.08\end{array}$ \\
\hline $\mathrm{f}$ & $\begin{array}{c}\psi_{s} \text { (MINIMOS) } \\
\% \text { difference }\end{array}$ & $3.70 \times 10^{-11}$ & $4.35 \times 10^{-11}$ \\
$\mathrm{~g}$ & $\begin{array}{c}\mathcal{E}_{s i} \text { (MINIMOS) } \\
\% \text { difference }\end{array}$ & $\begin{array}{c}2.93 \times 10^{-11} \\
-20.75\end{array}$ & $\begin{array}{c}3.57 \times 10^{-11} \\
-17.99\end{array}$ \\
\hline $\mathrm{h}$ & $\begin{array}{c}\mathcal{E}_{o x} \text { (MINIMOS) } \\
\% \text { difference }\end{array}$ & $3.69 \times 10^{-11}$ & $4.35 \times 10^{-11}$ \\
& \multicolumn{2}{|c|}{${ }^{2.20}$} & +0.05 \\
\hline
\end{tabular}

Table 3.1.2 Comparison of $Q_{G}$ determined by various methods. $L_{e s f}=2.3 \mu \mathrm{m}$; $V_{D S}=2.0 \mathrm{~V}, V_{S B}=0 \mathrm{~V} ;$ Differences taken with respect to $\psi_{\bullet}($ MINIMOS). 
by raising it near the source and drain slightly, thus making the charge induced on the gate somewhat smaller than that predicted from 1D analysis. Part of the difference is expected to be due to some numerical discrepancy between the two nethods of calculation, however, this appears to be very small since the trend in the values of $Q_{G}$ obtained from MINIMOS, both in the saturation and triode regions, clearly reveals the influence of short channel effects. The difference in $Q_{G}$ between methods (a) and (b) in Table 3.1.1 is therefore expected to increase, when considering the increased influence of $2 \mathrm{D}$ edge effects of the shorter $2.3 \mu \mathrm{m}$ device. Indeed, an increase of approximately five times the difference is observed in Table 3.1.1(e) and (f), in the case of the smaller device.

The gate charge evaluated using the transverse electric field distribution $\mathcal{E}_{s i}(y)$ in the silicon just under the oxide boundary (i.e. $Q_{G}\left(\mathcal{E}_{\mathbf{s i}}\right)$ ) produced very different results compared with those previously calculated using $\psi_{s}(y)$, as shown by Table 3.1.1(c) and Table 3.1.2(g). The difference between $Q_{G}\left(\psi_{s}\right)$ and $Q_{G}\left(\mathcal{E}_{s i}\right)$ obtained from MINIMOS is found to be over $24 \%$ for both regions of operation of the large device. The reason for such a large discrepancy was initially not understood, so further investigations were necessary.

Values of $\mathcal{E}_{s i}$ at variol:s points along the channel obtained from simulation were compared with those calculated from LCT, which were evaluated from the distributions of charge densities in the device; that is,

$$
Q_{G}=\int_{0}^{L} \epsilon_{s i} \mathcal{E}_{s i}(y) d y=\int_{0}^{L}\left(Q_{m}(y)+Q_{d e p}(y)\right) d y
$$

then at a point $y_{o}$ in the channel,

$$
\mathcal{E}_{s i}\left(y_{0}\right)=\frac{Q_{m}\left(y_{0}\right)+Q_{d e p}\left(y_{o}\right)}{\epsilon_{s i}}
$$

where,

$$
Q_{\text {dep }}(y)=-\sqrt{2 q \epsilon_{s i} N_{A} \psi_{s}(y)}
$$




$$
Q_{m}(y)=Q_{\imath}(y)-Q_{\text {dep }}(y)
$$

and where,

$$
Q_{s}(y)=-C_{o x}\left(V_{G B^{\prime}}-\psi_{s}(y)\right)
$$

The results listed in Table 3.2 show that the values of $\mathcal{E}_{s i}(y)$ from MINIMOS are consistently lower than those calculated from long channel theory, for both sets of simulations (i.e. in saturation and triode). The differences in $\mathcal{E}_{s i}(y)$ between simulation and long channel theory seem cousistent with those obtained for $Q_{G}$ evaluated from $\mathcal{E}_{s i}(y)$ and $1 \mathrm{D}$ theory.

\begin{tabular}{|c|c|c|c|c|}
\hline & $y(\mu \mathrm{m})$ & $\begin{array}{c}\mathcal{E}_{i i}(y)(\mathrm{V} / \mathrm{cm}) \\
(\mathrm{MINIMOS})\end{array}$ & $\begin{array}{c}\mathcal{E}_{i i}(y)(\mathrm{V} / \mathrm{cm}) \\
(\mathrm{LCT})\end{array}$ & $\begin{array}{c}\% \\
\text { difference }\end{array}$ \\
\hline & 0.594 & $1.55 \times 10^{5}$ & $2.23 \times 10^{5}$ & 30.40 \\
$V_{G S}=3.35 \mathrm{~V}$ & 21.98 & $1.47 \times 10^{5}$ & $1.94 \times 10^{5}$ & 24.23 \\
(Saturation) & 43.121 & $1.36 \times 10^{5}$ & $1.48 \times 10^{5}$ & 8.42 \\
& 48.72 & $8.30 \times 10^{4}$ & $1.20 \times 10^{5}$ & 30.83 \\
\hline & 2.696 & $1.75 \times 10^{5}$ & $2.46 \times 10^{5}$ & 28.98 \\
$V_{G S}=3.75 V$ & 20.284 & $1.63 \times 10^{5}$ & $2.19 \times 10^{5}$ & 25.50 \\
(Triode) & 43.127 & $1.36 \times 10^{5}$ & $1.63 \times 10^{5}$ & 16.41 \\
& 47.55 & $1.22 \times 10^{5}$ & $1.40 \times 10^{5}$ & 13.11 \\
\hline
\end{tabular}

Table 3.2 Comparison of $\mathcal{E}_{s i}$ by MINIMOS and long channel theory. $V_{D S}=2.0 \mathrm{~V}$, $V_{S B}=0 \mathrm{~V} ; L_{\text {eff }}=49.3 \mu \mathrm{m}$.

Upon further investigation, it was determined by comparison with hand calculations, that MINIMOS evaluates $\mathcal{E}_{s i}$ at every grid point directly from the potential 
$\psi$; that is

$$
\mathcal{E}_{\mathrm{ai}}(y)=\left.\frac{\Delta \psi(y)}{\Delta x}\right|_{y=y_{i}}
$$

where the difference in potential $\Delta \psi$ is calculated at every point $y_{i}$ along the channel between two adjacent grid points in the vertical $x$-direction from the surface of the device, $\Delta x$ being the distance between these two points. Calculations of $\mathcal{E}_{s i}(y)$ using (3.9) agreed very well with those given by MIN'MOS as shown in Table 3.3. It seems that the small discrepancies between the two sets of values in Table 3.3 may be due to the difference in the decimal accuracy between MINIMOS and the manual calculations.

\begin{tabular}{|c|c|c|c|}
\hline$y(\mu \mathrm{m})$ & $\begin{array}{c}\mathcal{E}_{s i}(\mathrm{~V} / \mathrm{cm}) \\
\text { equation (3.9) }\end{array}$ & $\begin{array}{c}\mathcal{E}_{s i}(\mathrm{~V} / \mathrm{cm}) \\
\text { (MINIMOS) }\end{array}$ & $\begin{array}{c}\% \\
\text { difference }\end{array}$ \\
\hline 0.5102 & $1.56 \times 10^{5}$ & $1.52 \times 10^{5}$ & +2.31 \\
18.6778 & $1.50 \times 10^{5}$ & $1.49 \times 10^{5}$ & +0.61 \\
40.1506 & $1.28 \times 10^{5}$ & $1.30 \times 10^{5}$ & -1.56 \\
45.1756 & $1.22 \times 10^{5}$ & $1.20 \times 10^{5}$ & +1.64 \\
47.1584 & $1.11 \times 10^{5}$ & $1.14 \times 10^{5}$ & -2.70 \\
48.7219 & $8.33 \times 10^{4}$ & $8.30 \times 10^{4}$ & +0.36 \\
48.9187 & $5.56 \times 10^{4}$ & $5.97 \times 10^{4}$ & -7.37 \\
\hline
\end{tabular}

Table 3.3 Comparison of $\mathcal{E}_{s i}$ by MINIMOS and potential differencing. $L_{e f f}=$ $49.3 \mu \mathrm{m} ; V_{G S}=3.35 \mathrm{~V}, V_{D S}=2.0 \mathrm{~V}, V_{S B}=0 \mathrm{~V}$; Saturation.

The third method used to evaluate $Q_{G}$ from MINIMOS output was by integrating the transverse electric field distribution $\mathcal{E}_{o x}(y)$ in the oxide, just above the $\mathrm{SiO}_{2} / \mathrm{Si}$ interface. the results of which, as shown in Table 3.1.1(d) and Table 3.1.2(h) agreed very well with both results for $Q_{G}\left(\psi_{s}\right)$, obtained from simulation 
and long channel theory.

Since $Q_{G}\left(\mathcal{E}_{s i}\right)$ and $Q_{G}\left(\mathcal{E}_{o x}\right)$ do not agree, it appears that the electric displacement through the $\mathrm{SiO}_{2} / \mathrm{Si}$ interface is not continuous. This is indeed shown to be the case from the results of Table 3.4, where the ratio $\left.\left(\mathcal{E}_{o x} / \mathcal{E}_{s i}\right)\right|_{y=y_{0}}$, taken at different points $y_{0}$ along the length of the channel, is consistently higher than the theoretical value given by

$$
\frac{\mathcal{E}_{o x}}{\mathcal{E}_{s i}}=\frac{\epsilon_{s i}}{\epsilon_{o x}}=\frac{11.9}{3.9}=3.05
$$

\begin{tabular}{|c|c|c|c|}
\hline$y_{o}(\mu m)$ & $\mathcal{E}_{o x}\left(y_{0}\right)(V / c m)$ & $\mathcal{E}_{s i}\left(y_{0}\right)(V / c m)$ & $\frac{\varepsilon_{o x}\left(y_{o}\right)}{\varepsilon_{s i}\left(y_{o}\right)}$ \\
\hline 8.86 & $6.26 \times 10^{5}$ & $1.54 \times 10^{5}$ & 4.06 \\
25.29 & $5.50 \times 10^{5}$ & $1.44 \times 10^{5}$ & 3.82 \\
35.10 & $4.85 \times 10^{5}$ & $1.36 \times 10^{5}$ & 3.57 \\
45.176 & $3.91 \times 10^{5}$ & $1.20 \times 10^{5}$ & 3.26 \\
\hline
\end{tabular}

Table 3.4 Comparison of $\mathcal{E}_{o x}$ and $\mathcal{E}_{a i} . L_{e f f}=49.3 \mu \mathrm{m} ; V_{G S}=3.35 \mathrm{~V}, V_{D S}=2.0 \mathrm{~V}$, $V_{S B}=0 V ;$ Saturation.

This can also be observed by looking at the distributions of $\mathcal{E}_{o x}(y)$ and $\mathcal{E}_{s 1}(y)$, shown in Figure 3.3. It is clear that the ratio of the magnitude of both fields varies considerably from place to place along the channel, as they are never parallel, nor does the ratio equal the theoretically expected value anywhere in the channel. Also shown in Figure 3.3 is the curve $\mathcal{E}_{s i}(y) \mathcal{E}_{o x}=\mathcal{E}_{o x}(y) \frac{\operatorname{cox}_{0 i}}{\mathcal{E}_{0 i}}$ which shows the true value of $\mathcal{E}_{s i}(y)$ based on $\mathcal{E}_{o x}(y)$ from MINIMOS, assuming the continuity of the electric displacement. A comparison of $\mathcal{E}_{s i}(y)_{\mathcal{E}_{o x}}$ with $\mathcal{E}_{s i}(y)$, serves as a measure of the difference in the electric displacement along the channel and of the discrepancy between $Q_{G}\left(\mathcal{E}_{o x}\right)$ and $Q_{G}\left(\mathcal{E}_{s i}\right)$. 


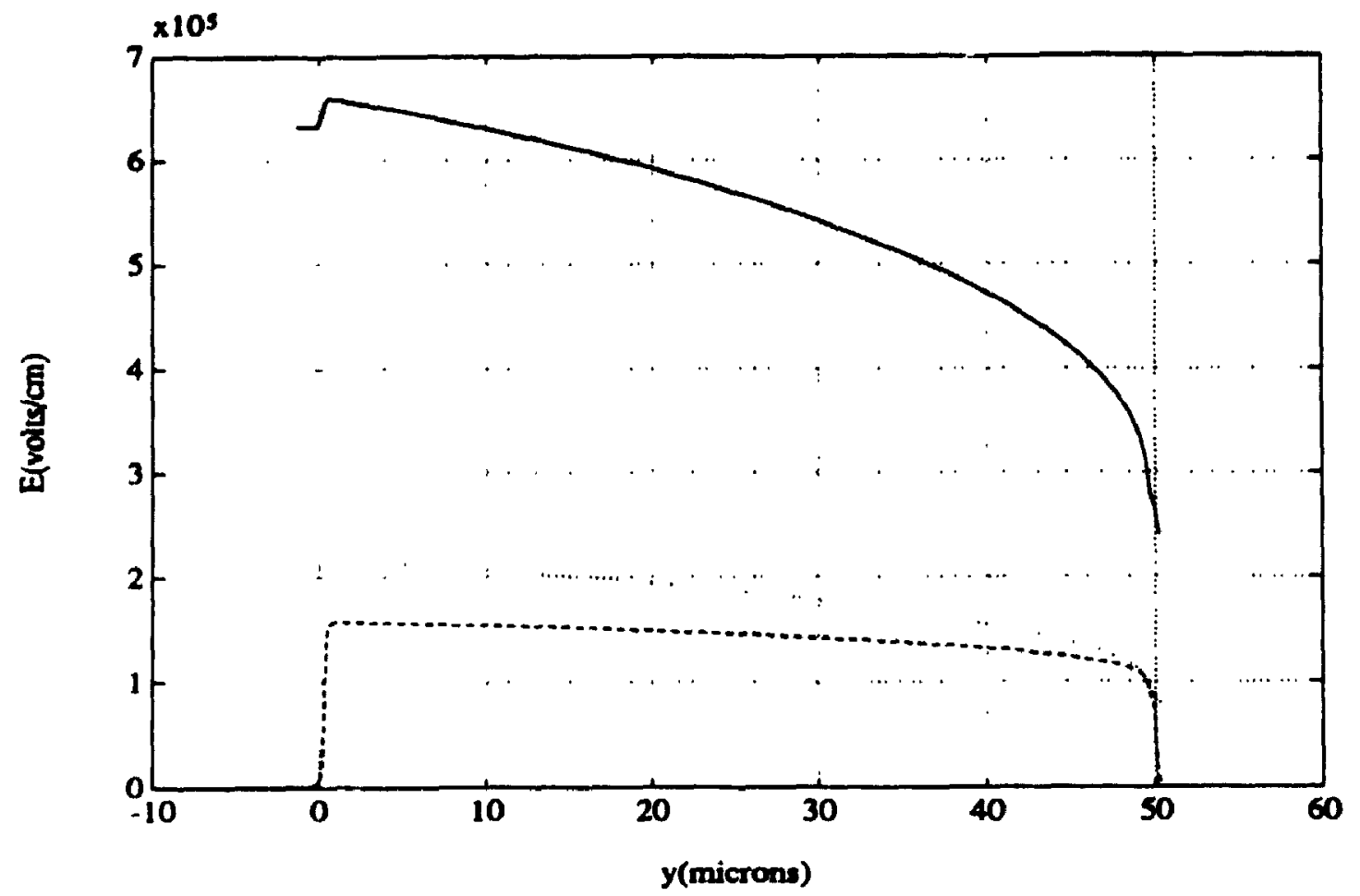

Figure 3.3 Distributions of $\mathcal{E}_{o x}(y)\left(\right.$ solid), $\mathcal{E}_{o i}(y)$ (dashed) and $\mathcal{E}_{o i}(y) \mathcal{E}_{o z}$ (dotted), for $V_{D S}=2.0 \mathrm{~V} ; L_{e f f}=49.3 \mu \mathrm{m}$

The accuracy of $\mathcal{E}_{o x}$ was also examined by comparing the electric displacement in the oxide at the $\mathrm{SiO}_{2} / \mathrm{Si}$ interface $\varepsilon_{o x} \varepsilon_{o x}\left(y_{0}\right)$, with that from silicon $\epsilon_{s} \mathcal{E}_{s}\left(y_{o}\right)$, based on LCT, at some point $y_{0}$ in the channel. From Table 3.4, at $y_{o}=25.29 \mu_{m}, \mathcal{E}_{o x}\left(y_{0}\right)=5.50 \times 10^{5}(\mathrm{~V} / \mathrm{cm})$. Using (3.6), $\mathcal{E}_{s i}\left(y_{o}\right)$ was evaluated to be $1.82 \times 10^{5}(\mathrm{~V} / \mathrm{cm})$ from LCT. Hence, the ratio

$$
\left.\frac{\mathcal{E}_{o x}\left(y_{o}\right)}{\mathcal{E}_{s i}\left(y_{o}\right)}\right|_{y_{0}=25.29 \mu \mathrm{m}}=\frac{5.50 \times 10^{5}(\mathrm{~V} / \mathrm{cm})}{1.82 \times 10^{5}(\mathrm{~V} / \mathrm{cm})}=3.02,
$$

producing a discrepancy of $0.98 \%$ from the expected theoretical ratio 3.05 , which corresponds well with the difference of $1.09 \%$ between $Q_{G}\left(\mathcal{E}_{0 x}\right)$ and $Q_{G}(\mathrm{LCT})$ in the saturation case given in Table 3.1.1. Hence, the value of $\varepsilon_{o x}$ given in the MINIMOS output was found to agree very well with LCT and $Q_{G}\left(\mathcal{E}_{a z}\right)$ gave almost identical results with $Q_{G}\left(\psi^{\prime}\right)$ from MINIMOS. 
In summary therefore, $Q_{G}$ was evaluated from three spacially dependent MINIMOS output quantities: $\psi_{\rho}(y), \mathcal{E}_{o x}(y)$ and $\mathcal{E}_{a i}(y)$, whose results were compared with $Q_{G}$ evaluated from ID theory. $Q_{G}\left(\psi_{s}\right)$ and $Q_{G}\left(\mathcal{E}_{o x}\right)$ were found to be in very good agreement with $Q_{G}(\mathrm{LCT})$, where the small differences were judged to be numerical and/or due to MINIMOS's representation of 2D effects, which are not included in long channel theory. $Q_{G}\left(\mathcal{E}_{a i}\right)$ however, does not agree with any of the other three methods. $\mathcal{E}_{\mathrm{Ai}}$ is an approximation of the surface field generated by MINIMOS from the potential difference between the first two vertically adjacent grid points; $\mathcal{E}_{s i}$ from MINIMOS represents the surface field but is not equal to it. Hence, it is fair to conclude at this point that $\mathcal{E}_{s i}(y)$ is not suitable for the purpose of calculating

$Q_{G}$. Both $\psi_{s}(y)$ and $\mathcal{E}_{0 x}(y)$ from MINIMOS proved to be sufficiently accurate to be used to evaluate $Q_{G}$ in the process of obtaining gate capacitances, however, $\psi_{s}(y)$ is the method chosen on grounds of simplicity. It was thought that $\mathcal{E}_{\text {ox }}(y)$, ring better able to represent 2D effects near the source and drain, would yield greater accuracy especially in the $2.3 \mu \mathrm{m}$ devics but since the results are nearly identical with those from $\psi_{s}(y)$ in every case, this was not observed.

\subsubsection{Interpretation of Results with $\mathcal{E}_{s i}$}

The reason why the electric displacement is not continuous at the $\mathrm{SiO}_{2} / \mathrm{Si}$ interface needs to be clarified. It was suspected that the discontinuity may be due to inherent limitations of MINIMOS in its treatment of specific physical properties of the MOSFET; specifically in treating the mobile charge density $Q_{m}(y)$, spread along the surface of the channel. Thus, it was necessary to investigate how $Q_{m}(y)$ was being considered by MINIMOS.

This investigation was done in two parts: 1) considering a long channei device in both saturation and triode regions of operation, $Q_{M}$ calculated from LCT was compared with the discrepancy between the electric displacements in the oxide and in the silicon, to see if MINIMOS was possibly treating the mobile charge as being located at the surface (as in the sheet channel approximation) so as 


$$
\epsilon_{o x} \mathcal{E}_{o x}=\epsilon_{a i} \mathcal{E}_{a i}+Q_{M}
$$

and 2) the continuity of electric displacement was investigated for a device operating in the subthreshold, where the mobile charge density is essentially zero $\left(Q_{m} \simeq 0\right)$.

To satisfy the first part of the investigation, the total rabile charge $Q_{M}$, calculated from long channel theory, for both saturation and triode regions is listed below in Table 3.5, as are the differences in gate charge calculated from $\mathcal{E}_{o x}(y)$ and $\mathcal{E}_{s t}(y)$, and from $\psi_{s}(y)$ and $\mathcal{E}_{s t}(y)$; that is:

$$
\begin{aligned}
& Q_{G}\left(\mathcal{E}_{o x}\right)-Q_{G}\left(\mathcal{E}_{s i}\right) \\
& Q_{G}\left(\psi_{s}\right)-Q_{G}\left(\mathcal{E}_{s i}\right)
\end{aligned}
$$

where

$$
\begin{gathered}
Q_{G}\left(\mathcal{E}_{o x}\right)=\int_{0}^{L} \epsilon_{o x} \mathcal{E}_{o x}(y) d y \\
Q_{G}\left(\mathcal{E}_{s i}\right)=\int_{0}^{L} \epsilon_{s i} \mathcal{E}_{s i}(y) d y \\
\left.Q_{G}\left(\psi_{s}\right)=C_{o x} \int_{0}^{L} V_{G B^{\prime}}-\psi_{s}(y)\right) d y
\end{gathered}
$$

\begin{tabular}{|c|c|c|c|}
\hline$V_{G S}(V)$ & $\begin{array}{c}Q_{m}(\mathrm{LCT}) \\
(\mathrm{coul} / \mathrm{cm})\end{array}$ & $\begin{array}{c}(3.11) \\
(\mathrm{coul} / \mathrm{cm})\end{array}$ & $\begin{array}{c}(3.12) \\
(\mathrm{coul} / \mathrm{cm})\end{array}$ \\
\hline 3.35 (Saturation) & $-5.22 \times 10^{-10}$ & $-2.187 \times 10^{-10}$ & $-2.51 \times 10^{-10}$ \\
\hline 3.75 (Triode) & $-6.11 \times 10^{-10}$ & $-2.442 \times 10^{-10}$ & $-2.797 \times 10^{-10}$ \\
\hline
\end{tabular}

Table 3.5 Comparison of $Q_{G}$ value.. $L_{e f f}=49.3 \mu m ; V_{D S}=2.0 \mathrm{~V}, V_{S B}=0 \mathrm{~V}$.

It was found that in both regions of operation, the calculated value of $Q_{M}$ was approxinately double the value given by (3.11) and (3.12). This seems to suggest 
that in MINIMOS, the grid point separation given as $18 A^{\circ}$, is comparable or less than the depth of the mobile charge distribution, which is indeed of the order 40$50 \AA$ [24]. Thus $\mathcal{E}_{s i}$ calculated from the potential difference between the first two grid points is an approximation and less than the true surface field. Equally, the value of $\mathcal{E}_{s i}$ underestimates $Q_{M}$ and $Q_{G}$.

It is to be expected that for the device operating in the subthreshold region, there should be much closer agreement between $\mathcal{E}_{s i}$ and $\mathcal{E}_{o x}$. This was examined with $V_{G S}=0.5 \mathrm{~V}$ and was found to be the case; the ratio $\mathcal{E}_{o x} / \mathcal{E}_{s i}$ was found to be consistently 3.11 along the total effective channel length, except near the drain. where it fell down to 3.08 , possibly due to numerical computation errors.

\subsection{Extracting Gate Capacitances}

\subsubsection{Introduction}

The various physical properties given by MINIMOS have been studied in the previous section to determine how well they agree with long channel theory for a device of large channel length and to examine the feasibility of using them as the basis of investigation of gate charge and associated capacitance properties of simulated devices. Short channel effects have also been observed and influence on the device characteristics noted.

It has been determined that the surface potential distribution $\psi_{s}(y)$ from MINIMOS is the most appropriate and practical source of data from which to evaluate the gate charge $Q_{G}$, though the transverse electric field distribution in the oxide $\mathcal{E}_{o x}(y)$ may prove as suitable (or perhaps even more so), in the case of short devices, in light of its apparent ability to represent short channel effects with more: sensitivity.

The different techniques that were used to carry out the required simulations in orc - to obtain viable results for evaluating the appropriate relationships for $Q_{G}\left(V_{i}\right) \sim V_{G S}$ and $C_{G i} \sim V_{G S}$, where $i$ represents each of the terminals $G, D$, 
$S$, and $B$, will be discussed in this section, as will the methods for processing the simulated data.

\subsubsection{Study of First Simulation Procedure}

The gate capacitance $C_{G i}$ for any dc bias condition is calculated from the difference in the values of integrated gate charge $\Delta Q_{G}$ for two MINIMOS simulations corresponding to incrementally different values of voltage $V_{i}, V_{i}+\Delta V$ at the device terminal $i$. The procedures and considerations of accuracy involved in obtaining $C_{G i} \sim V_{G S}$ characteristics from MINIMOS simulations in this way, are first discussed in the present section for the case of the gate self capacitance $C_{G G}$.

To obtain such a relationship requires that many simulations be performed in sequence, this being accomplished by changing the input gate voltage, represented as $U G$ in MINIMOS, by an increment $\Delta V_{G S}$, corresponding to $D G$ in MINIMOS. The increment $D G$ must be chosen to be as fine as possible, in order to produce a smooth continuous relationship of $Q_{G} \sim V_{G S}$ and therefore capacitance. $D G$ was limited by the simulator to a minimum value of $\pm 0.1 \mathrm{~V}$, which was used throughout the present work.

The input to MINIMOS consists of a set of instructions, which are identified by the type of IDENT. Some IDENTs are used to describe the device physically; i.e. $n$ or p-channel, thickness of the oxide, gate length, type of implant and dose, the kind of profile required, etc.. The impurity profile is crudely represented by Gaussian distributions. Other IDENTs are left to specify the operating conditions of the device while others control the outputs. The grid is defined internally by MINIMOS, and may be different for each simulation. STEP IDENT overrides the the automatic grid assignment (using the OPTION GRIDFREEZE), for up to ten successive simulations.

Simulations were performed over the range $-5.0 \leq V_{G S} \leq 5.0$ for every curve, in ten groups, with ten simulations per group and a $0.1 \mathrm{~V}$ increment. The outputs from each group of simulations were organized and processed using a Fortran program. 
The gate charge $Q_{G}$ was calculated at every point by integrating numerically the specified property (i.e. $\left.\psi_{a}(y), \varepsilon_{0 x}(y)\right)$ using (3.3) and (3.4).

The gate self-capacitance $C_{G G}$ was evaluated for every consecutive pair of gate charges by

$$
C_{G G}\left(\overline{V_{G S}}\right)=\frac{\Delta Q_{G}}{\Delta V_{G S}}=\frac{Q_{G_{i}}-Q_{G_{i+1}}}{V_{G S_{i}}-V_{G S_{i+1}}}
$$

where the subscripts correspond to points along the $Q_{G} \sim V_{G S}$ curve, which are spaced by the voltage interval $\Delta V_{G S}$ and $\nabla_{G S}$ is the average value of $V_{G S}$ at which $C_{G G}$ is evaluated; that is

$$
\overline{V_{G S}}=\frac{V_{G S_{1}}+V_{G S_{i+1}}}{2}
$$

The curve of the gate charge characteristics $Q_{G}\left(\mathcal{E}_{o x}\right) \sim V_{G S}{ }^{5}$ of the MD was evaluated by integrating $\mathcal{E}_{o x}(y)$ and is shown in Figure 3.4(a). The operating conditions were $V_{D S}=2.0 \mathrm{~V}, V_{S B}=0 \mathrm{~V}$. The relationship exhibits numerical scatter as well as several major discontinuities which can be observed roughly at one volt intervals. The latter are numerical errors associated with the change of grid pattern introduced by MINIMOS for each group of simulations. MINIMOS makes this decision based on the initial voltage conditions which have been specified for every simulation (or group of simulations). Hence, it will initiate a grid change accordingly, as it has been designed to provide a resolution in accordance with the conditions imposed upon it for the various regions of operation.

As a result of the sensitivity of $C_{G G}$ to the changes in $Q_{G} \sim V_{G S}$ expressed by (3.16), the numerical scatter of the results is amplified considerably as sern

5 Due to the loss of the original file, whose values extended throughout the rangr of gate voltages $-5.0 \leq V_{G S} \leq 5.0 \mathrm{~V}$, the values are instead shown to begin at $V_{G S}=-1.0 \mathrm{~V}$, whereas the $C_{G G}\left(\mathcal{E}_{0 x}\right) \sim V_{G S}$ characteristics are plotted throughout the entire sperifird range. 


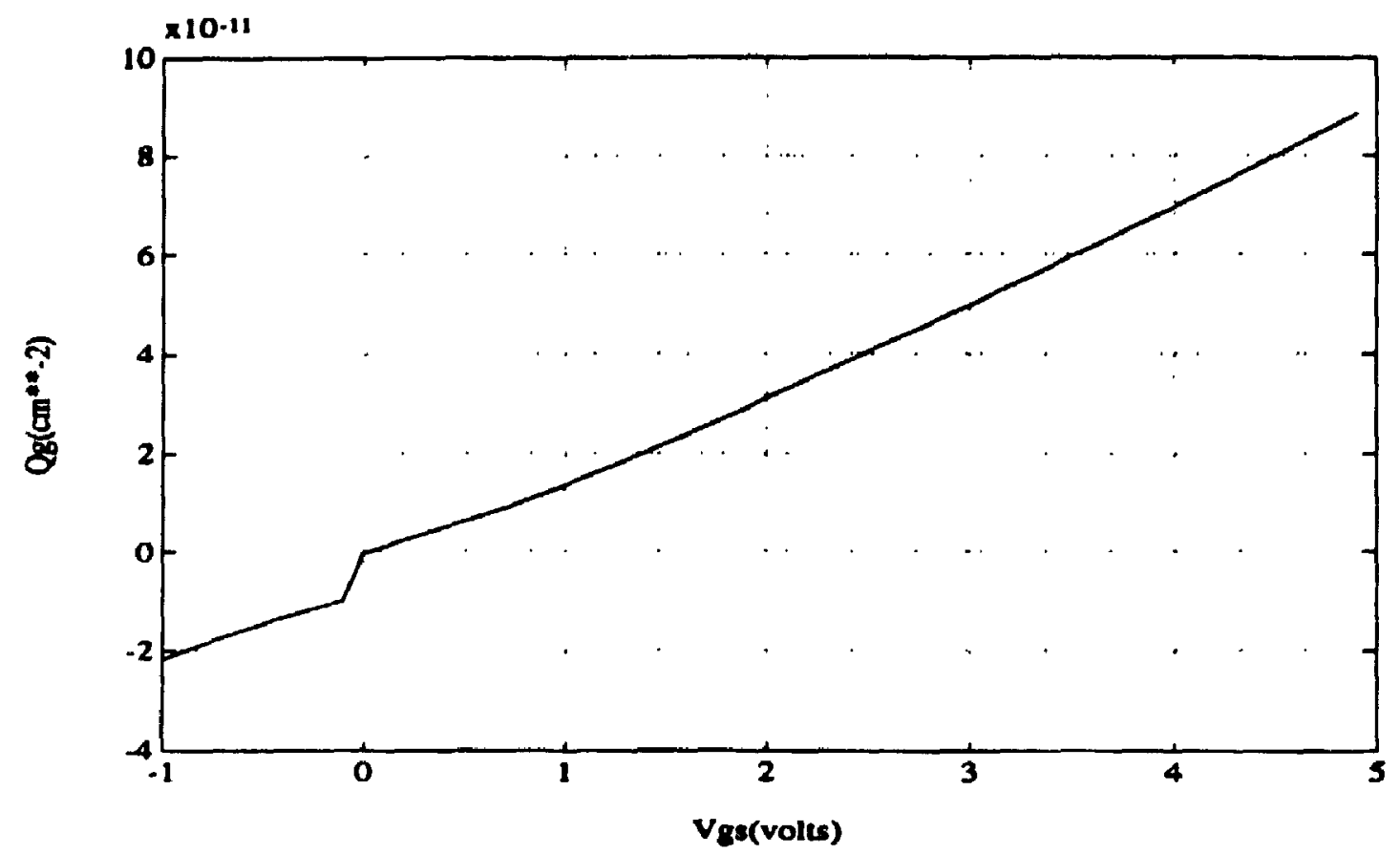

Figure 3.4(a) $Q_{G}\left(\mathcal{E}_{o x}\right) \sim V_{G S}$; gate charge $Q_{G}\left(\mathcal{E}_{o x}\right)$ is evaluated by integrating the transverse electric field inside the oxide $\mathcal{E}_{o x}$ for incremental gate voltages $\Delta V_{G S}=0.1 \mathrm{~V} ; V_{D S}=2.0 \mathrm{~V}, L_{e f f}=2.3 \mu \mathrm{m}$.

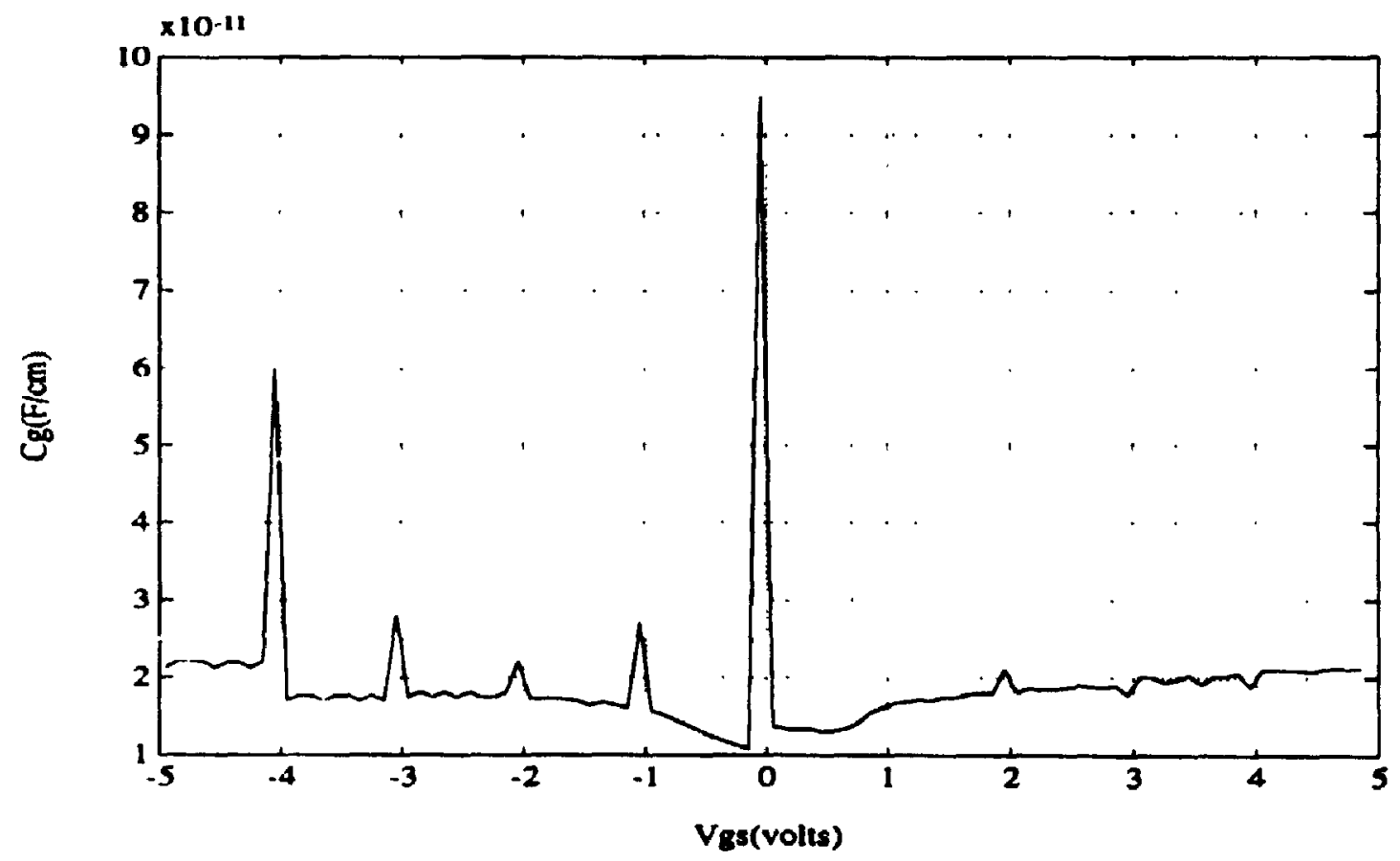

Figure 3.4(b) $C_{G G}\left(\varepsilon_{o x}\right) \sim l_{G S}$ characteristics evaluated using the first simulation proced ure showing all the discontinuities; $C_{G G}\left(\mathcal{E}_{o x}\right)$ is evaluated by taking $د Q_{G}\left(\varepsilon_{o x}\right) / \Delta V_{G S} ; V_{D S}=2.0 \mathrm{~V}, L_{\text {eff }}=2.3 \mu \mathrm{m}$. 


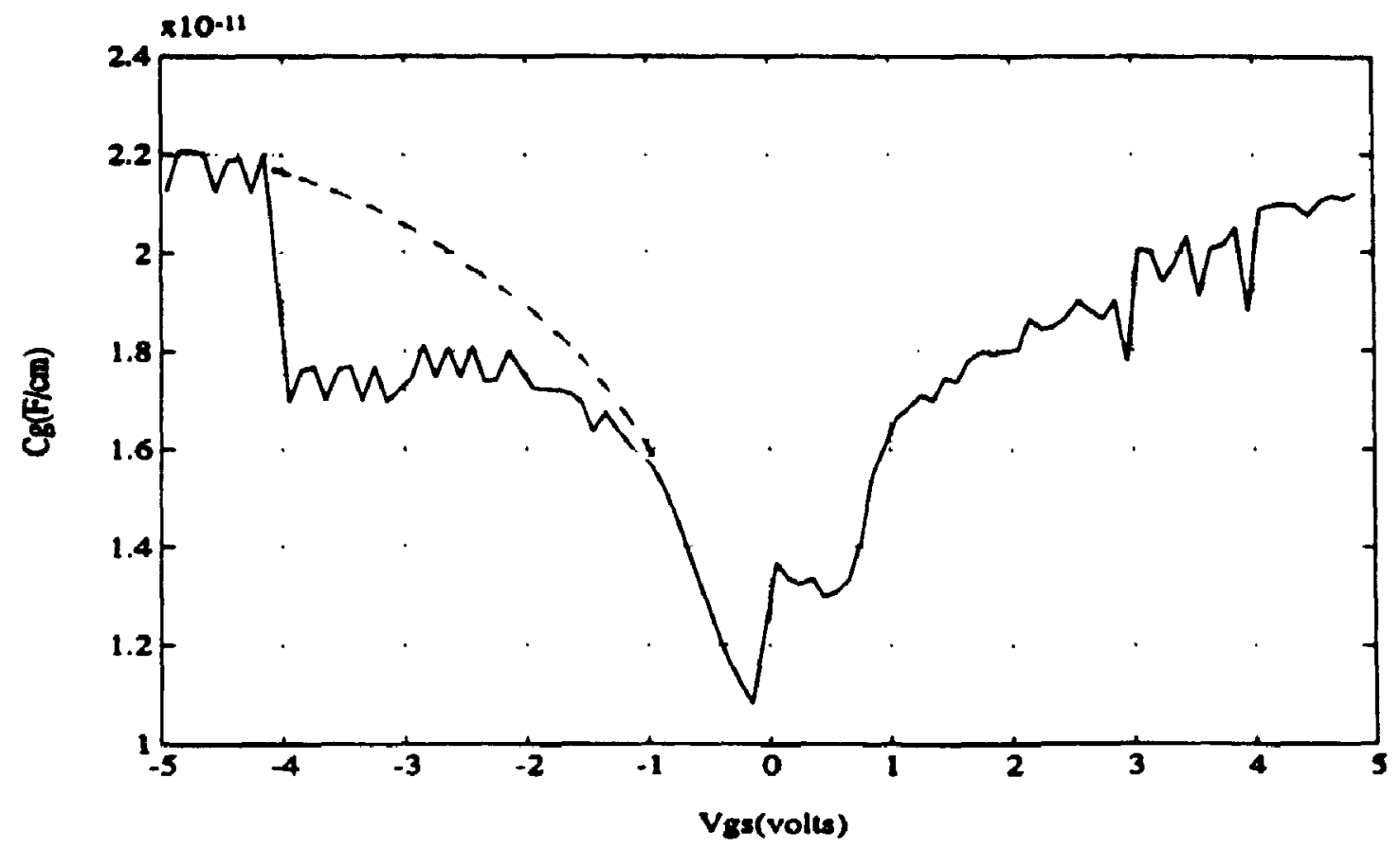

Figure 3.4(c) $C_{G G}\left(\mathcal{E}_{o x}\right) \sim V_{G S}$ characteristics evaluated using the first simulation procedure after removing the major discontinuities.

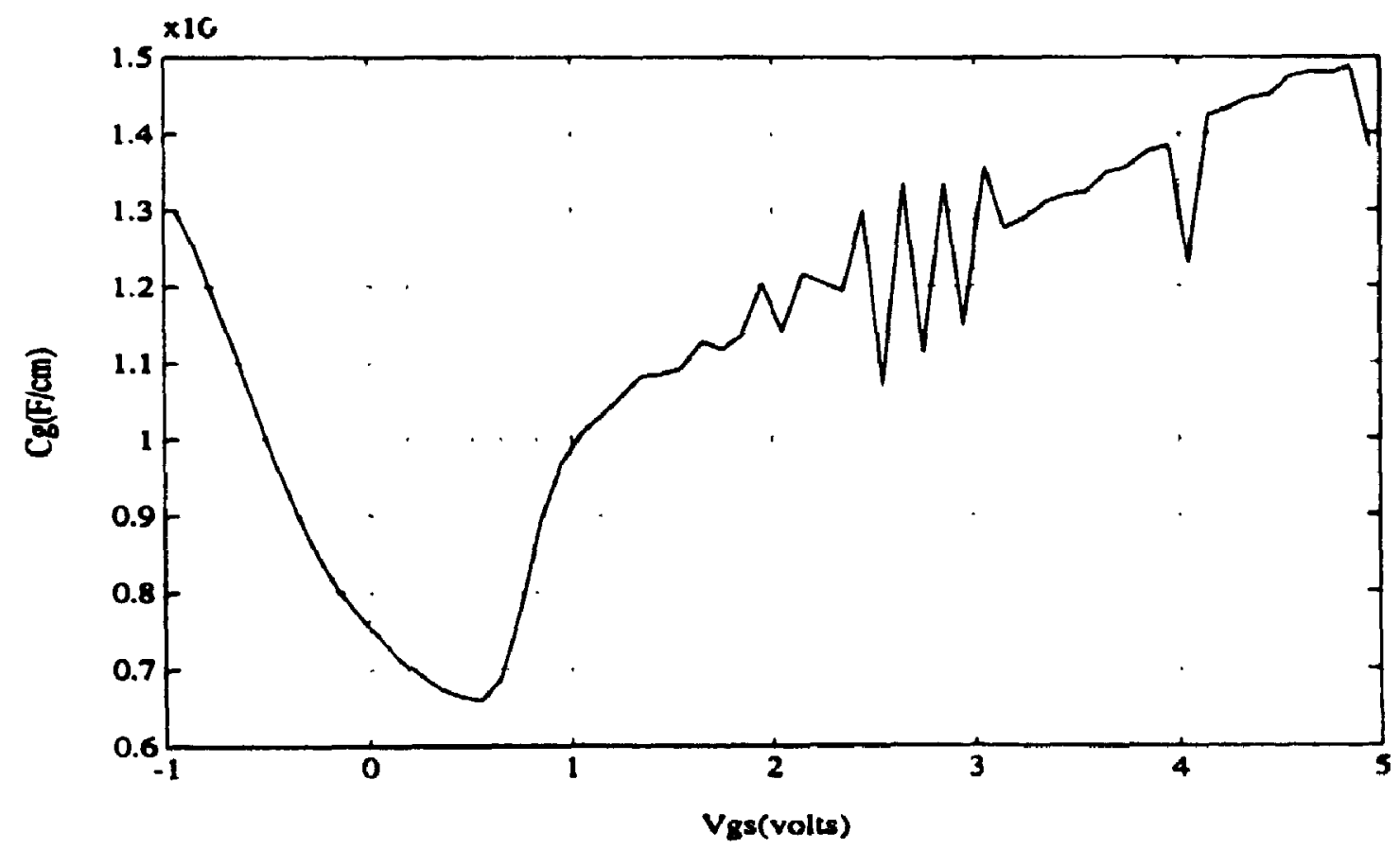

Figure 3.5 $C_{G G}\left(\psi_{\mathrm{s}}\right) \sim V_{G S}$ characteristics evaluated using the first simulation procedure after all major discontinuities have been removed; $C_{G G}\left(\psi_{0}\right)$ is evaluated by taking $\Delta Q_{G}\left(\psi_{s}\right) / \Delta V_{G S} ; V_{D S}=2.0 \mathrm{~V}, L_{e f f}=2.3 \mu \mathrm{m}$. 
in Figure 3.4(b). Intelligible information is considerably suppressed due to the discontinuities caused by the change of grid, but upon closer observation the rough form of the $C_{C G}$ capacitance curve may be observed. After removing these points of discontinuity (manually), $C_{G G}$ takes on a much more familiar appearance; see Figure 3.4(c). However, considerable scatter remained with some large jumps in the capacitance still present, raising serious questions regarding the accuracy of the capacitance characteristics obtainable from MINIMOS. It is noted that major discrepancies are present in the accumulation region ${ }^{6}$ of operation, the expected behavior being indicated by the broken line.

Based on skepticism in regard to the simulation accuracy of MINIMOS in the accumulation region, it was decided to examine the characteristics beyond this region, namely, in the subthreshold, saturation and triode regions, which are in any case of more practical significance.

The $C_{G G}\left(\psi_{s}\right) \sim V_{G S}$ characteristics for the same device and operating bias conditions are shown in Figure 3.5, with all the major discontinuities removed. Capacitance is obtained by differencing the gate charge, evaluated by integrating the surface potential in the channel of the device. The behavior of the capacitance characteristics shown in Figure 3.5 are in reasonable agreement with those based on $\mathcal{E}_{o x}(y)$ shown in Figure 3.4(c) ${ }^{7}$ for $V_{G S} \geq 0.5 \mathrm{~V}$. Below this value $\psi_{s}(y)$ appears to give more accurate and smooth results.

\footnotetext{
6 The originators of MINIMOS[26] were aware of its shortcomings in the accumulation region and had proposed to remedy these problems in future papers, however, that has not been realized.

7 The ralues of the capacitances of Figure 3.4(c) and Figure 3.5 are not in agreement, however, because the data of Figure 3.4(c) was initially processed incorrectly, giving the wrong vertical scale. Proper comparison of Figure $3.4($ c $)$ with $C_{G G}\left(\psi_{\mathrm{o}}\right) \sim V_{G s}$, evaluated under the same erroneous conditions, was made at the time, however, that file was accidently lost. The purpose of the comparison is served however, the essential thing being, to note the shape of the curves and the presence of scatter.
} 


\subsubsection{Study of Improved Simulation Procedure}

Results obtained from the previous sub-section showed that capacitance characteristics could indeed be extracted using MINIMOS, though with considerable numerical scatter present, provided that the accumulation region is excluded, for which MINIMOS simulations are unreliable.

However, unnecessary numerical scatter was observed in the $C_{G G}$ characteristics, based on data of both $\psi_{s}$ and $\mathcal{E}_{o z}$, at points where MINIMOS initiated a change in grid pattern. It was realized that a great deal of scatter could be eliminated from the curves if a way could be found to hold the grid fixed over the complete range of simulations required for each curve. MINIMOS provides the command GRIDFREEZE for just such a purpose, however, the number of points necessary (i.e. the number of simulations) was much greater than ten, the permissible maximum of the simulator. Through a series of trial and error simulations, it was discovered that MINIMOS decided upon the resolution of the grid based on the magnitude of the combination of input biases; that is, the higher the combination of voltages, the finer the resolution of the grid, since that meant the presence of more intense electric field effects and greater changes to consider near the edges and on the surface of the device. It was necessary therefore to begin the set of simulations with the largest combination of infut voltages followed by progressively reducing the inputs for the subsequent simulations by the required interval, thus covering the necessary range of bias conditions. When the grid was not chosen by imposing the most demanding voltage conditions first, it was found that MINIMOS did not carry out all of the required simulations of that set using a fixed grid, but rather chose to change it in order to meet the conditions, despite the input request for GRIDFREEZE.

The objectives therefore, were to choose a simulation pattern for each curve, such that it does not exceed ten simulation points per group of simulations, making certain that the starting point defines the most severe bias conditions in the curve. This meant, starting the set of simulations with the highest value of $V_{G S}$, so that 
MINIMOS would choose the correct grid pattern to begin with and hop down to lower gate voltages for the following simulations in the group.

One other condition that was imposed by MINIMOS was the size of the hopping interval of the terminal voltages should not exceed $\pm 5.0 \mathrm{~V}$ or the accuracy may suffer. This of course meant that the voltage range of each curve could not exceed five volts; a rather tight range indeed, for which the characteristics would not even cover the operating range of voltages expected of MOS transistors in many modern circuit applications. Trial sets of simulations were made to observe the effects on results, covering a range greater than the five volts limited by MINIMOS and it was found in practice that a range of seven volts could be used without incurring a change of grid.

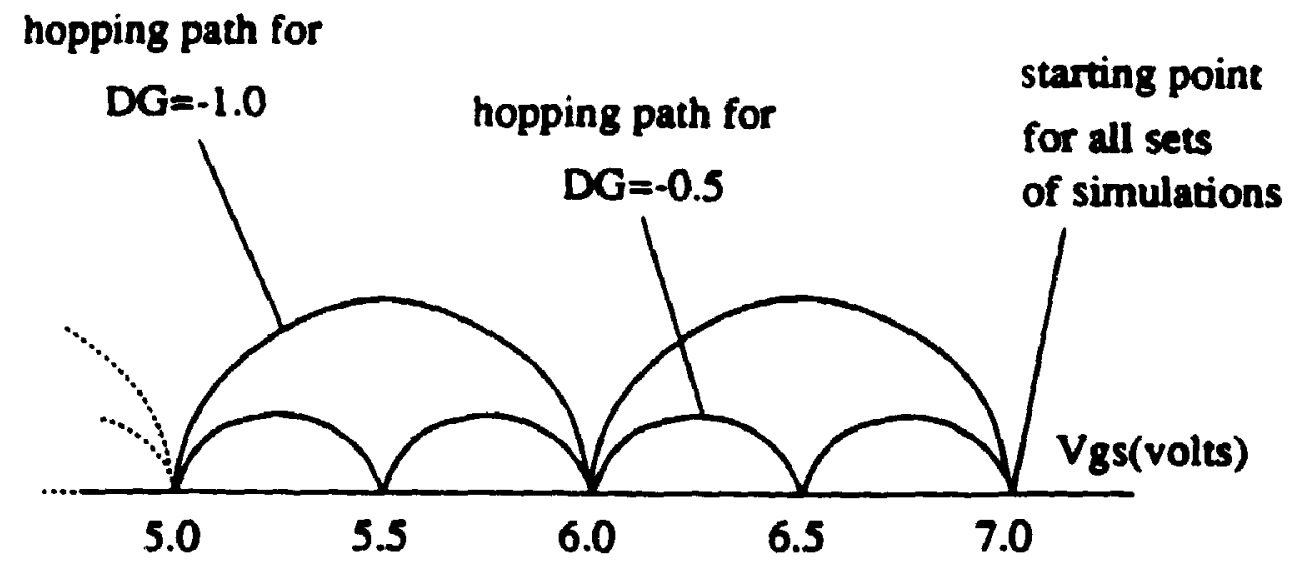

Figure 3.6 Illustration showing the method by which the range of $V_{G S}$ is segmented into the different sets of hopping intervals indicated by DG, in order to include all the necessary points of simulation with $\Delta V_{G S}=0.1 \mathrm{~V}$.

Using the above approach it was decided to derive the capacitance characteristics, plotted against $V_{G s}$, for three drain voltages (i.e. $V_{D S}=0.1 \mathrm{~V}, 2.0 \mathrm{~V}, 4.0 \mathrm{~V}$ ), for each of the gate capacitances and for each of the three devices of different effective channel lengths. In order to obtain a high enough resolution of the $Q_{G} \sim V_{G S}$ curves, simulations were made at $0.1 \mathrm{~V}$ intervals between points, as in the previ- 
ous subsection. The actual procedure used to make the necessary simulations over the given range of gate voltages for each curve, while holding the grid frozen, was different for each of the gate capacitances $C_{G G}, C_{G D}, C_{G S}, C_{G B}$.

The easiest of the four groups of characteristics to obtain was $C_{G G}$, since it only required hopping paths in $V_{G S}$ to cover all the necessary points; see Figure 3.6. It was a simple matter to evaluate $C_{G G}$ from the corresponding integrated $Q_{G}$ data for each of the three curves using (3.16).

Obtaining the sets of simulations for $C_{G D}$ was not as straight forward, however. If the required curve had been plotted versus $V_{D S}$, the procedure would have been similar to $C_{G G} \sim V_{G S}$, since

$$
C_{G D}=\frac{\Delta Q_{G}}{\Delta V_{D S}}
$$

however, in order to plot $C_{G D} \sim V_{G S}$ it would be necessary to make simulations spanning essentially three dimensions (i.e. one for each of $Q_{G}, V_{G S}, V_{D S}$ ); that is, it was required to simulate a surface of points of $Q_{G}$, shown in Figure 3.7(a), rather than being a function of one variable. However, instead of spanning the simulations completely in the third dimension (the $V_{D S}$ axis, in this case), thus generating a lot of useless data and wasting much computing time, simulations were only made for the range of $\Delta V_{D S}$ that the curves for $C_{G D}$ would occupy, such that $\Delta V_{D S}=V_{D S 2}-V_{D S 1}$ where $V_{D S 1}=0.1 V, V_{D S 2}=0.2 V$ for the $C_{C i D}$ curve at $V_{D S}=0.15 \mathrm{~V}^{8}$. Likewise, the curves $V_{D S}=2.0 \mathrm{~V}$ and $V_{D S}=4.0 \mathrm{~V}$ had the corresponding simulation points for $V_{D S 1} / V_{D S 2}$ at $: 1.95 \mathrm{~V} / 2.05 \mathrm{~V}$ and $3.95 \mathrm{~V} / 4.05 \mathrm{~V}$ respectively, over the whole range of $V_{G S}$. Hence, $Q_{G}$ was evaluated twice for every point of $V_{G S}$ (i.e. at $V_{D S 1}$ and $V_{D S 2}$ ) for each of the three curves, thus requiring roughly double the number of simulations (i.e. computing time) to obtain $C_{C D D}$

${ }^{8}$ MINIMOS demands that $(U D-U S) \geq 0.1 V$, hence, the first simulation point was chosen at $V_{D S}=0.1 \mathrm{~V}$ and the second at $V_{D S}=0.2 \mathrm{~V}$, where $V_{S B}=0.0 \mathrm{~V}$; hence, for the case of $C_{G D}$ and $C_{G S}$, this curve was chosen by actually taking the average at $V_{D S}=0.15 \mathrm{~V}$. 


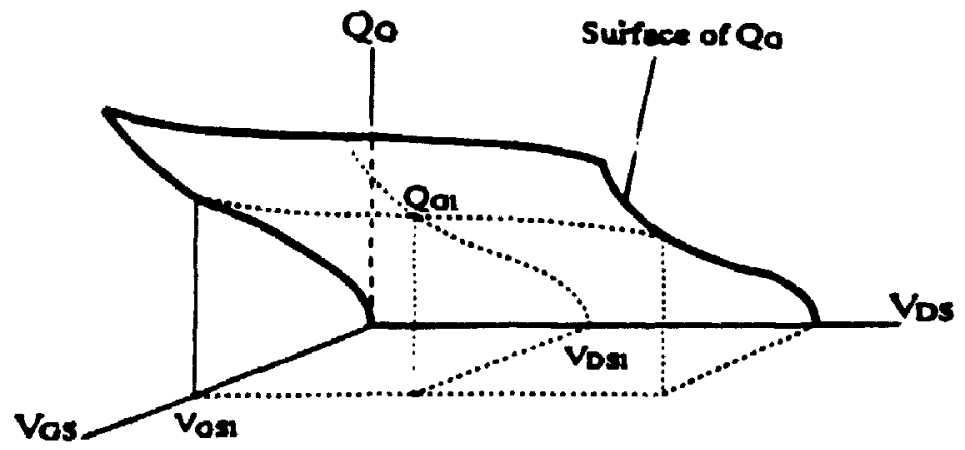

(a)

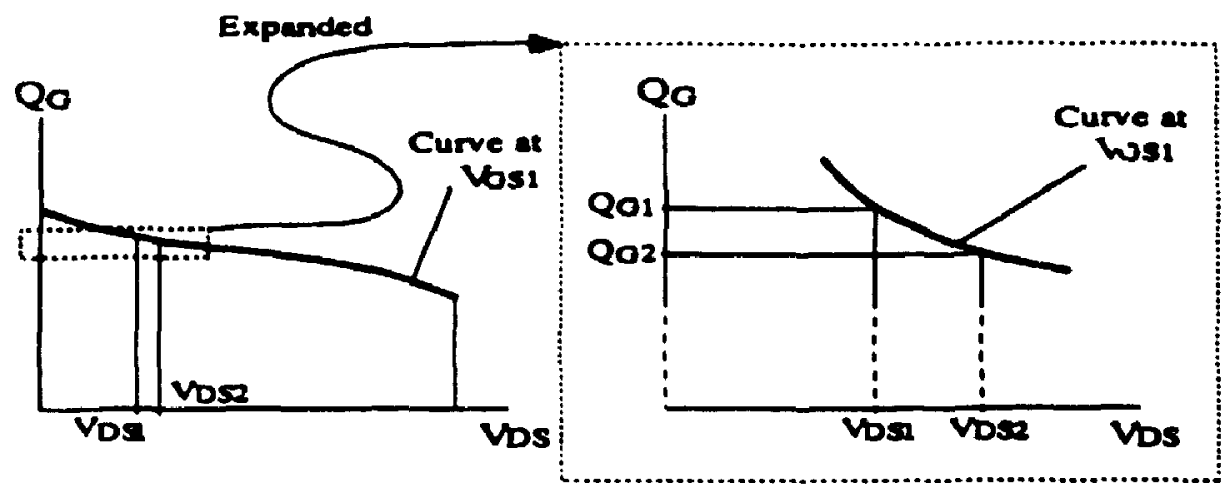

(b)

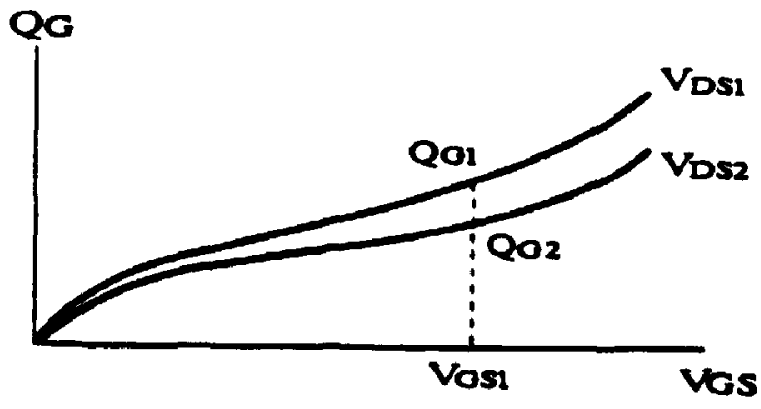

(c)

Figure 3.7

a) 3D view of surface of $Q_{G}$ points, of which the curve of all $Q_{G 1}$ values is shown for $V_{D S_{1}}$; b) 2D projection of the surface of $Q_{G}$ points on the $Q_{G} \sim V_{D S}$ axis, cut at $V_{G S 1}$, with the expanded area showing the two values of $Q_{G 1}$ and $Q_{G 2}$ at $V_{D S 1}$ and $V_{D S 2}$, respectively; c) $2 D$ projection of both $Q_{G}$ curves on $Q_{G} \sim l_{G S}$ axis. at $Y_{D S 1}$ and $V_{D S 2}$. 


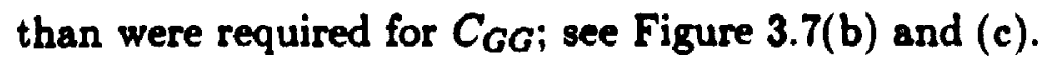

The situation was similar for $C_{G S}$. However, there was an added complication by MINIMOS: no intervals of $V_{S B}$ were permitted in the simulation sets; i.e. $V_{S B}$ could not be changed directly, because the source is taken as reference. Hence, in order to get both sets of simulation points $V_{S B 1}$ and $V_{S B 2}$, all of the three other terminals (gate, drain and substrate) had to be adjusted instead; that is, by raising all of the other three terminals up by $\Delta V_{i}$, it was equivalent to decreasing $V_{S B}$ by $\Delta V_{S B}$, provided that the bulk potential never exceeded that of the source for any simulation. This was the most time consuming of all the gate capacitances to obtain, of which the actual number of simulations for each curve of $C_{G S}$ required approximately five times the number required for each $C_{G G}$ curve.

Finally, the sets of simulations for $C_{G B}$ required a relatively simple simulation path, compared to $C_{G S}$. Hopping between simulation points was made by simply varying voltages $V_{B S}$ for every point along the total range of $V_{G S}$, i.e. for every point along $V_{G S}$, there were two simulations taken; that is, $V_{B S 1}$ and $V_{B S 2}$ corresponding to the two gate charges $Q_{G 1}$ and $Q_{G 2}$. 


\section{Chapter 4 \\ Discussion of Results of Capacitance Studies}

In this chapter the gate self-capacitance $C_{G G}$ is first discussed, after which the properties determined for its components $C_{G D}, C_{G S}$ and $C_{G B}$ are exa:nined; these are ubtained from MINIMOS simulation using the improved method discussed in Chapter 3 for long (LD), medium (MD) and short devices (SD).

The behavior of the derived capacitance characteristics is analyzed by considering two chief determining factors: the effects on the characteristics due to limitations of MINIMOS, appearing as scatter of data and the influence of the physical mechanisms on the dynamic behavior of the MOSFET, as the dimensions of the device are reduced.

The effect of the scatter of gate charge data provided by MINIMOS on the rapacitances is often found to be so great as to make the interpretation of the results difficult. Thus, curve fitting of the gate charge data is attempted to provide a means of smoothing the capacitance characteristics and is treated after the analysis of the accuracy of the capacitance data.

The gate capacitances for the LD are then examined, with the object of establishing correlation of the simulation-derived capacitance properties with well known long channel characteristics, before proceeding to the results obtained for the smaller devices. 


\subsection{Capacitance Data from MINIMOS Simulation}

\subsubsection{Accuracy of Results}

The accuracy of the results obtained from MINIMOS simulation is discussed

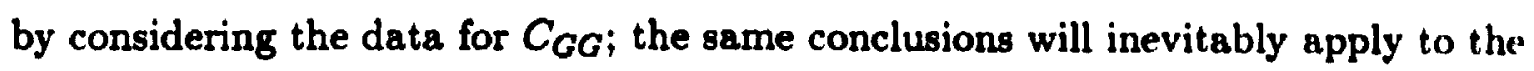
results obtained for the gate capacitance components $C_{G D}, C_{G S}$ and $C_{G B}$.

The improved simulation technique discussed previously (Section 3.3.3) resulted in significantly better results compared to those of the first method of simulntion (Section 3.3.2); however, there were still considerable errors introduced by MIN IMOS, resulting in somewhat rough $Q_{G} \sim V_{G S}$ curves, as may be observed from Figure 4.1(a). This roughness of the gate charge data is translated into considerable scatter in $C_{G G} \sim V_{G S}$, due to the sensitivity of capacitance to changes in charge (see Figure 4.1(b)). Even for those curves of gate charge that appear to be smooth, such as Figure 4.3(a) for the SD, there is significant scatter in the corresponding curves of $C_{G G} \sim V_{G S}$, shown in Figure 4.3(b). The uncertainty in the characteristics is often large enough to cloud the true behavior of the device under particular conditions.

Results for the LD are examined first with the expectation of verifying agreement with long channel theory. In the linear region, with increasing $V_{G S}, C_{G G}$ is expected to approach the value of gate oxide capacitance $C_{o}=L_{e f f} \cdot W \cdot \epsilon_{v x} / T_{o x}=$ 1.7pF. This is indeed the case as may be seen from Figure 4.1(b); it is particularly clear from the curve at $V_{D S}=0.1 \mathrm{~V}$, which is very smooth.

The minimum value of $C_{G G}$ in the subthreshold region is closely the same for all three values of drain voltage and the value of $0.67 p F$ at the threshold voltage agrees quite well with the value of $0.697 p F$ calculated from LCT.

The accuracy of results for $C_{G G}$ for $V_{D S}=2.0 \mathrm{~V}$ and $4.0 \mathrm{~V}$ is more uncertain in the saturation region, as shown by the large degree of nu:nerical scaticr. Simulations were made for each curve in saturation, without the option GRIDFREEZE at $V_{G S}=$ 


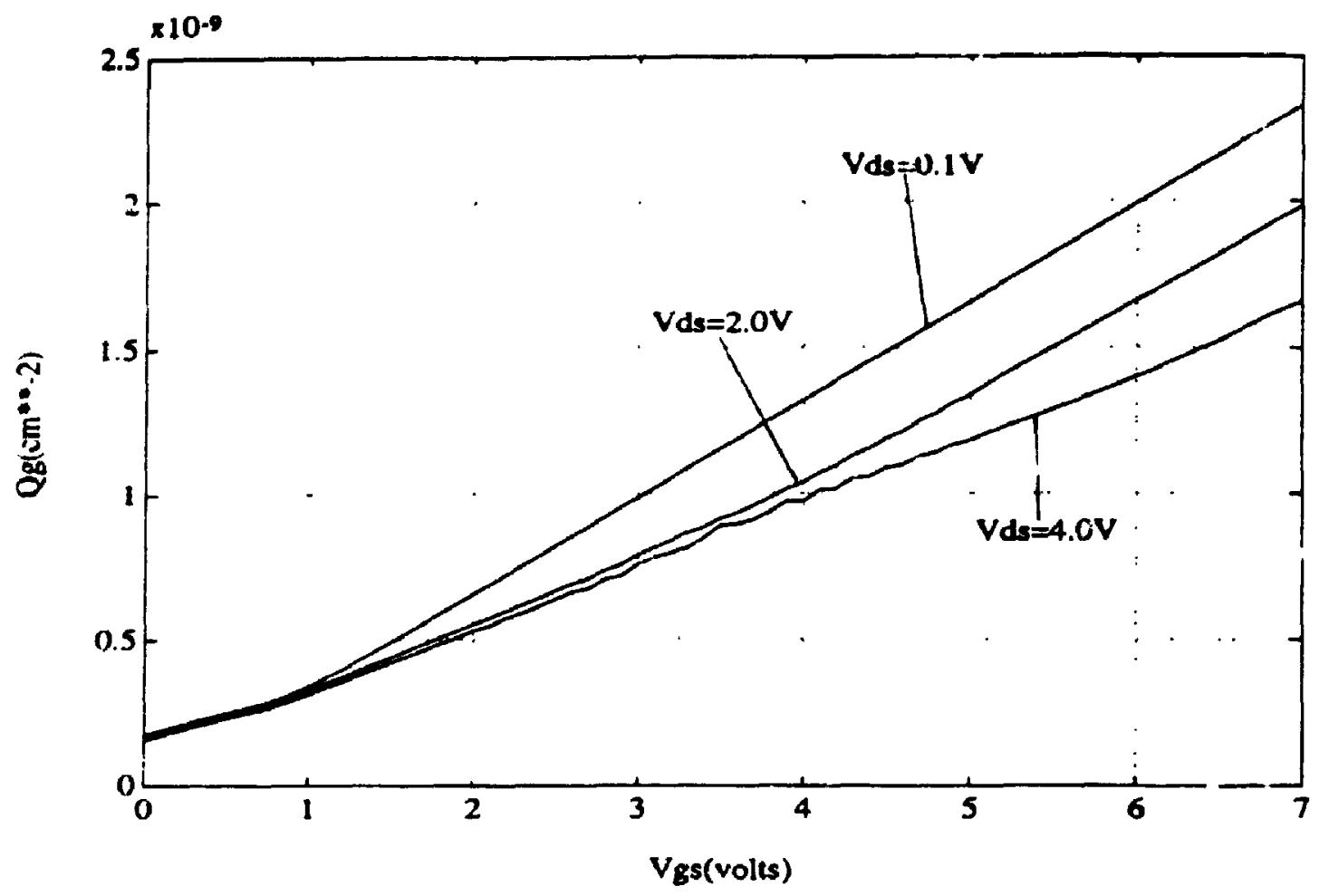

Figure 4.1(a) $\quad Q_{G} \sim V_{G S} ; L_{e f f}=49.3 \mu \mathrm{m}$

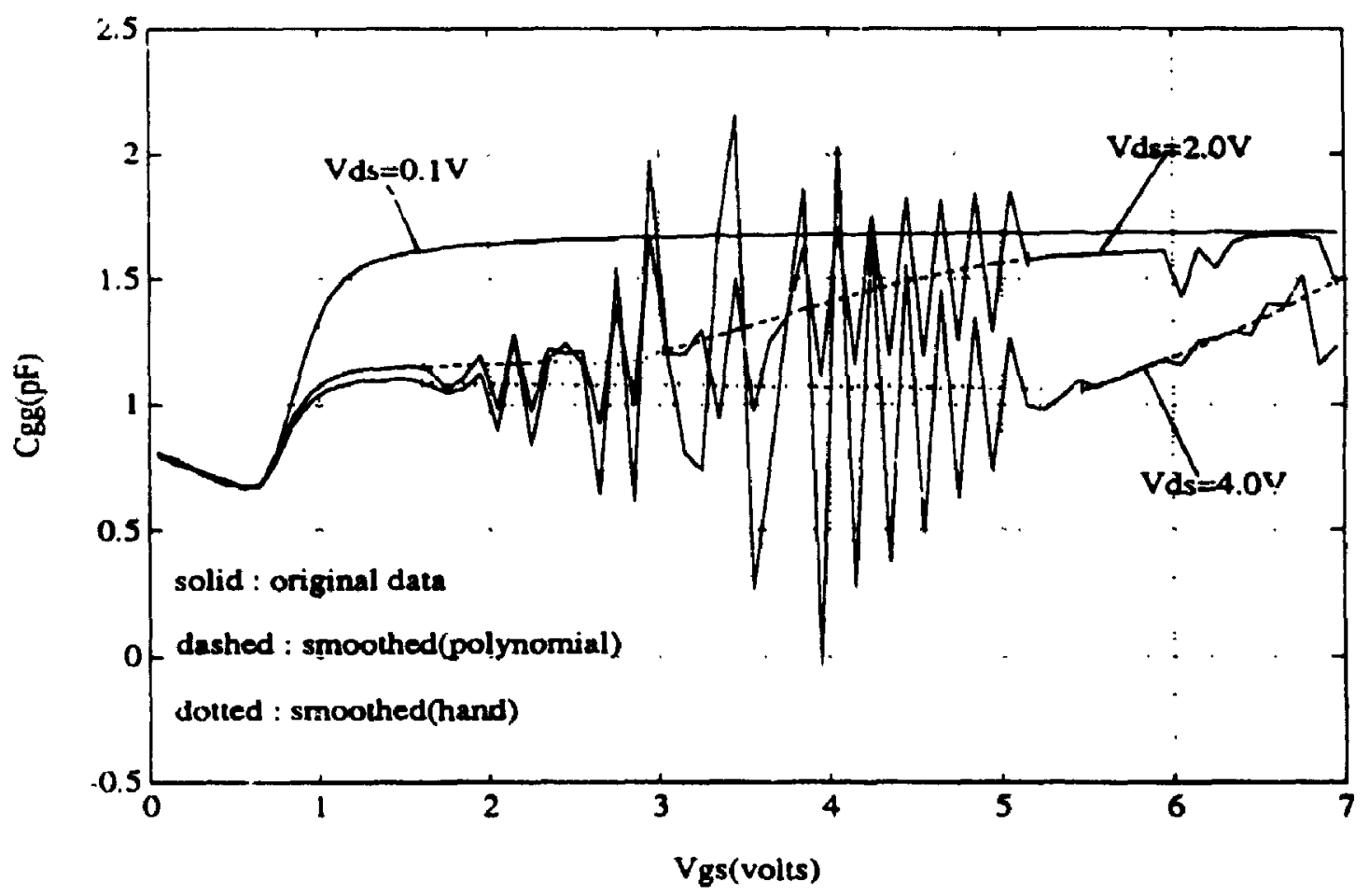

Figure 4.1(b) $\quad C_{G G} \sim V_{G S} ; L_{e f f}=49.3 \mu \mathrm{m}$. 
$3.2 \mathrm{~V}$ and $5.0 \mathrm{~V}$ for $V_{D S}=2.0 \mathrm{~V}$ and $4.0 \mathrm{~V}$ respectively, where the long channel pinchoff points are calculated from LCT at $V_{\text {gsat }}=3.54 \mathrm{~V}$ and $6.0 \mathrm{~V}$ respectively. Though these simulations would introduce their own numerical errors in the evaluation of $C_{G G}$, it was thought that the accuracy would be better than with the procedure employing the option GRIDFREEZE, since the mesh pattern was chosen to suit specific bias conditions and not those over a whole range of terminal bias inputs. This could also provide a measure of the penalty paid, if any, by employing the option GRIDFREEZE.

$C_{G G}$ without GRIDFREEZE, evaluated to be $1.07 p F$ at $V_{G S}=3.2 \mathrm{~V}$ and $V_{D S}=$ $2.0 \mathrm{~V}$, does not agree very well with the value of $1.25 p F$, from Figure $4.1(\mathrm{~b})$, and $1.28 p F$ from LCT; however, it falls somewhere inside the envelope of numerical scatter. Thus, $C_{G G}$ evaluated at one discrete point, without the GRIDFREEZE option apnears to represent a more accurate value of the device behavior and can perhaps serve as a guide for such purposes as curve fitting (to be discussed later), over those results suggested by the envelope of numerical scatter from the curve.

The same can not be concluded about the value calculated at $V_{G S}=5.0 \mathrm{~V}$ and $V_{D S}=4.0 \mathrm{~V}$. Clearly the device is operating in the saturation region since $V_{\text {gsat }}=$ $6.0 \mathrm{~V}$, yet the value of $1.45 p F$ obtained for $C_{G G}$ without GRIDFREEZE is well above the margin of behavior suggested by the envelope of scattered points and the value obtained from $L C T$; it even surpasses the value of $C_{G G}$ at $V_{G S}=7.0 \mathrm{~V}$, where the device is in the triode region. The simulator cannot be trusted for accurate results in saturation at high drain voltage bias conditions, this being evident by the range of uncertainty of $C_{G G}$ for the curve $V_{D S}=4.0 \mathrm{~V}$. It seems that the values of $C_{G G}$ obtained using GRIDFREEZE for these bias conditions represents the best accuracy MINIMOS is capable of, because the mesh pattern selected for the initial conditions, which also represent the most demanding conditions over the whole ange of simulatious, provides the best resolution possible. The accuracy of results obtained from MINIMOS for large drain biases is poor in either case, but the option GRIDFREEZE, used in the manner described in Section 3.3.3, does help to reduce 
the amount of numerical uncertainty ${ }^{1}$. Thus, besed on the $C_{G G}$ results for the LD, MINIMOS simulations are disappointingly inaccurate as a basis of capacitance determination.

Turning to the $C_{G G}$ characteristics obtained for $\mathrm{t}$. : medium length device MD, shown in Figure 4.2(b), it is noted that there is con Jerable reduction in numerical scatter, compared with the LD case, as may be 'spected from the increased resolution of mesh points in the channel and judginf trom the $Q_{G} \sim V_{C S}$ relationships in Figure 4.2(a). In the triode region the curves all correctly approach the gate oxide rapacitance $C_{o}=79.42 f F$.

Even less scatter can be noted from the $Q_{G} \sim V_{G S}$ and $C_{G G} \sim V_{G S}$ characteristics of the SD, from Figures 4.3(a) and 4.3(b) respectively. In this case $C_{o}=34.53 f F$ and found to be the limiting value for all three curves in the triode region. However, the curves for $V_{D S}=2.0 \mathrm{~V}$ and $V_{D S}=4.0 \mathrm{~V}$ appear suspiciously close together; they effectively overlap from around threshold and do not exhibit the expected separation of the MD and LD characteristics in the saturation and linear regions.

Evaluations of $C_{G G}$ for the SD without GRIDFREEZE were made for various values of $V_{G S}$ and both values of $V_{D S}$, to investigate if the above peculiarity is a result of keeping the grid fixed. Results evaluated at $V_{G S}=0.4 \mathrm{~V}, 2.0 \mathrm{~V}$ and $6.0 \mathrm{~V}$ compared with $C_{G G}$ from the curves are listed in Table 4.6. The values of $C_{G G}$ at $V_{G S}=0.4 \mathrm{~V}$ and $2.0 \mathrm{~V}$ agree quite well with the curves, both being within the numerical scatter range given for each curve; however, the values obtained from discrete simulations at $V_{G S}=6.0 \mathrm{~V}$ are clearly too large, producing errors as large as thuse of either curve; for the case of $V_{D S}=4.0 \mathrm{~V}$, both methods of simulation (with and without GRIDFREEZE) fall short of any reasonable result, due to the limited

\footnotetext{
1 Because of the finer resolution of mesh points using GRIDFREEZE, the accuracy of the values of $Q_{G}$ is improved over the complete range of $V_{G S}$, except for those points simulated around the initial bias conditions, whose marked increase in scatter noted in every capacitance curve, is due again to the insufficient number of mesh points chosen by MINIMOS for the initial bias conditions of the simulation.
} 


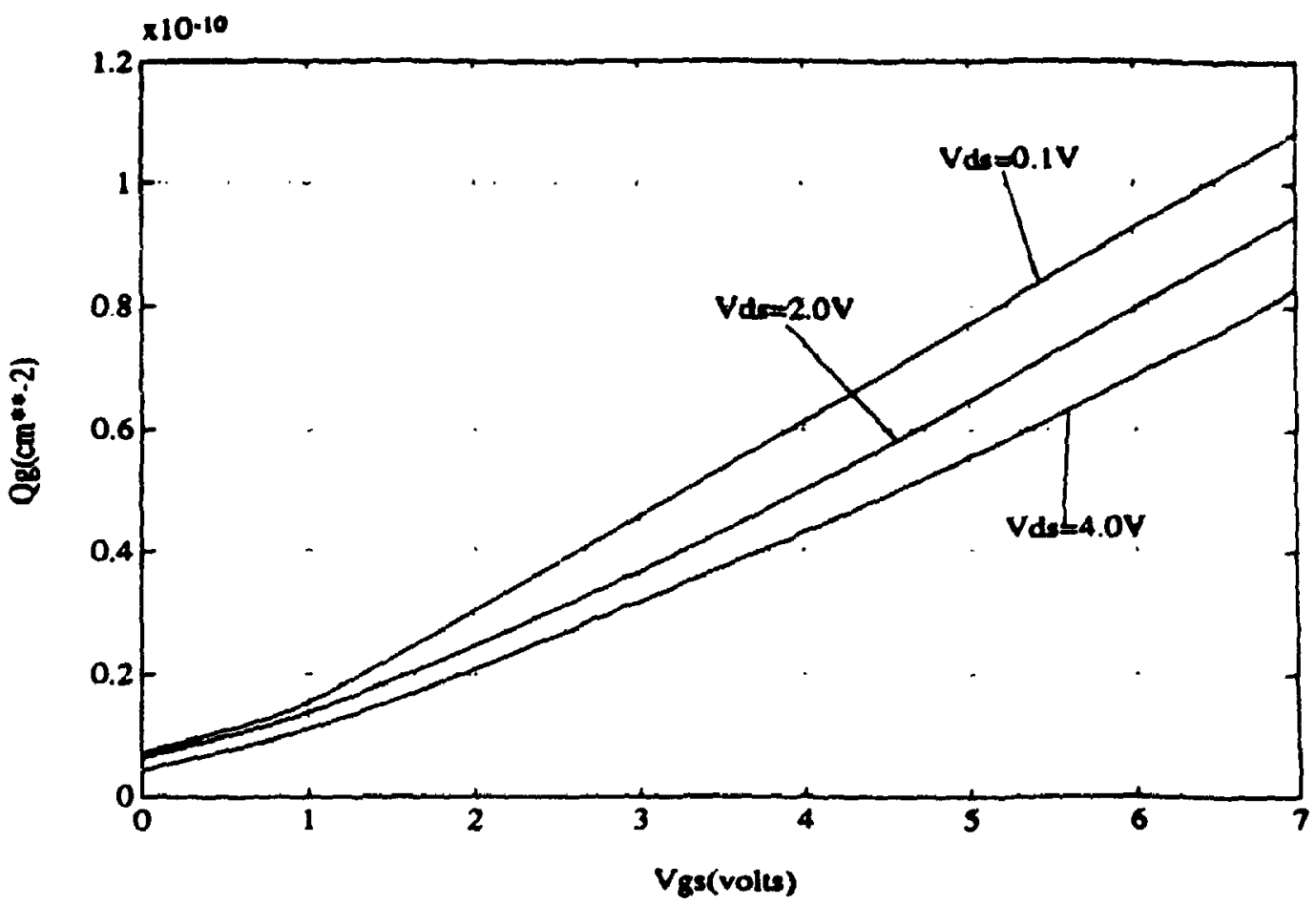

Figure 4.2(a) $\quad Q_{G} \sim V_{G S} ; L_{e f f}=2.3 \mu \mathrm{m}$.

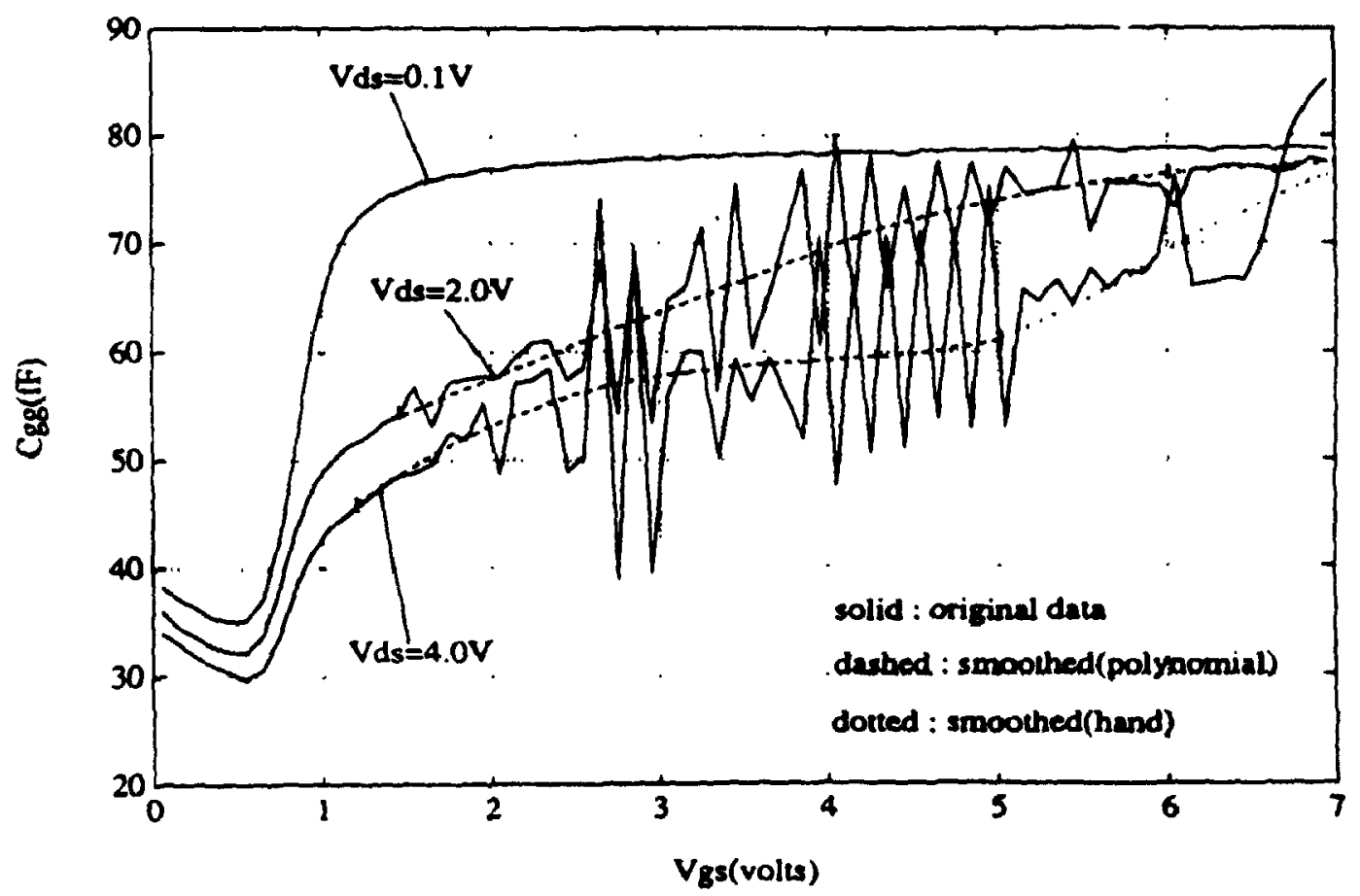

Figure 4.2(b) $\quad C_{G G} \sim V_{G S ;} L_{e f f}=2.3 \mu \mathrm{m}$ 


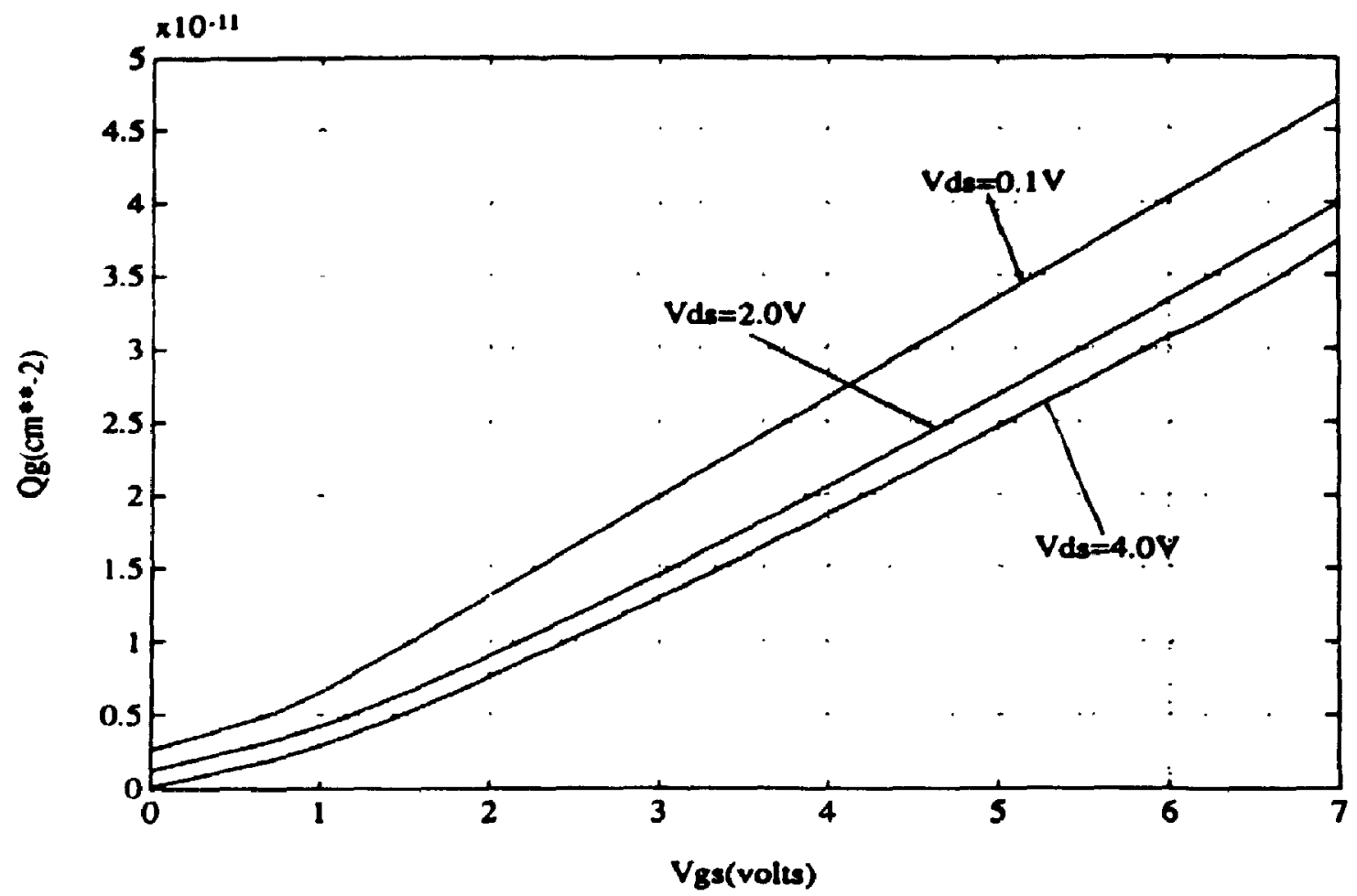

Figure 4.3(a) $\quad Q_{G} \sim V_{G S} ; L_{\text {eff }}=1.0 \mu \mathrm{m}$.

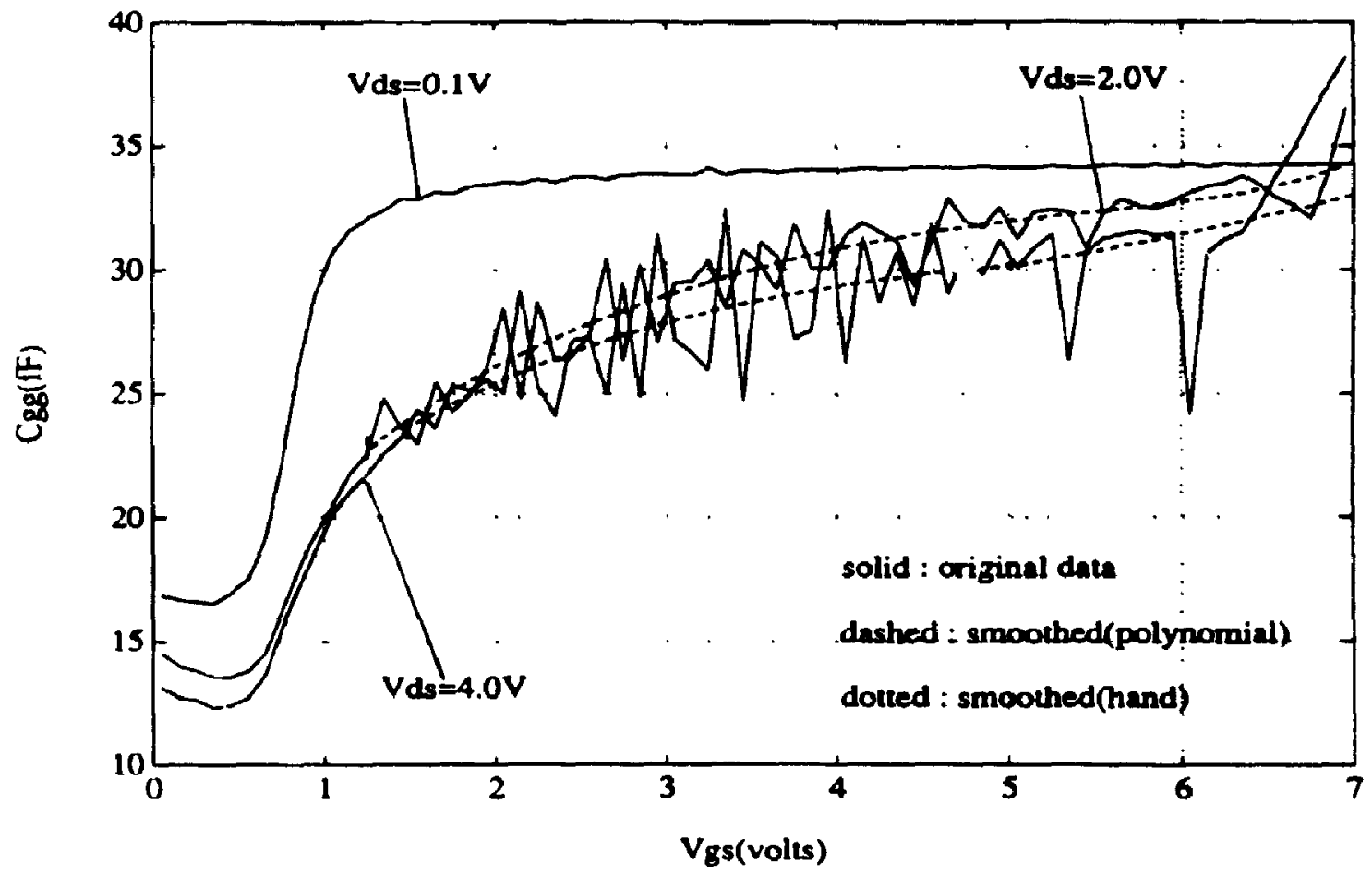

Figure 4.3(b) $\quad C_{G G} \sim V_{G S} ; L_{\text {eff }}=1.0 \mu \mathrm{m}$. 
resolution of the grid chosen by MINIMOS to cope with large potential gradients around saturation. The results obtained by employing the option GRIDFREEZE have proved to be more accurate in all other cases tested. Judging from these results, and the relatively small scatter of the data, it would seem that the curve for $V_{D S}=4.0 \mathrm{~V}$ would be at least as accurate as the other devices at the same drain voltage. At this stage, it is concluded that the resu ts did represent the true behavior of short channel capacitances under these bias conditions. These results, however, are considered further in a broader context, after a collective analysis of all the curves.

\begin{tabular}{|c|c|c|c|c|}
\hline$V_{G S}(V)$ & $\begin{array}{c}V_{D S}=2.0 \mathrm{~V} \\
C_{G G}(f F) \\
\text { No GRDFR2 }\end{array}$ & $\begin{array}{c}V_{D S}=2.0 V \\
C_{G G}(f F) \\
\text { Figure 4.5 }\end{array}$ & $\begin{array}{c}V_{D S}=4.0 \mathrm{~V} \\
C_{G G}(f F) \\
\text { No GRDFR2 }\end{array}$ & $\begin{array}{c}V_{D S}=4.0 \mathrm{~V} \\
C_{G G}(f F) \\
\text { Figure 4.5 }\end{array}$ \\
\hline 0.4 & 13.08 & 13.55 & 12.30 & 12.35 \\
2.0 & 26.85 & 27.175 & 27.50 & 25.30 \\
6.0 & 36.35 & 32.95 & 36.34 & 27.75 \\
\hline
\end{tabular}

Table 4.6 Comparison of $C_{G G}$ evaluated from simulation without gridfreeze and from Figure $4.5 ; L_{e f f}=1.0 \mu \mathrm{m}$.

The degree of numerical scatter varies with operating region for each of the capacitance characteristics, but it was basically found to be largest for simulations of the LD, operating at high bias conditions in saturation. It is believed that the uncertainty in the results from the simulator, represented as numerical scatter is an inherent feature of MINIMOS and is due to: 1) the limited number of mesh points available and 2) the way in which these points are distributed in the automatic mesh generation. MINIMOS was dusigned as a short channel device analyzer, hence, the number of grid points it must distribute inside the channel is limited by an algorithm designed to satisfy both simulation accuracy and time, within some error margin. When making simulations of large devices, MINIMOS can not provide 
the necessary resolution of grid points around the drain area where large potential gradients occur, since a certain number of points must be spread along the length of the channel, and this will result in inaccuracies at the output. This is best demonstrated by the roughness of the $Q_{G}$ characteristics and the corresponding scattered $C_{G G}$ characteristics, for $V_{D S}=4.0 \mathrm{~V}$ in Figure 4.1(a) and 4.1(b). Under less demanding circumstances, such as when simulating lower drain voltages and/or smaller devices, the grid resolution is adequate around the edges and surface of the device and allows for smoother gate charge and capacitance characteristics, as shown in Figures 4.1(a) and 4.1(b) for $V_{D S}=0.1 \mathrm{~V}$ and $V_{D S}=2.0 \mathrm{~V}$, and in Figures 4.2(a), (b) and 4.3(a), (b).

\subsubsection{Smoothing of Data}

In most cases $Q_{G} \sim V_{G S}$ and hence, $C_{G i} \sim V_{G S}$ data extracted from MINIMOS exhibit large scatter due to numerical errors, masking the true behavior of the capacitance characteristics. Because of the sensitivity of $C_{G i}$ to changes in $Q_{G}$, it was considered worthwhile to attempt to reduce the effects of numerical scatter of gate charge data points by smoothing, before evaluating capacitance by taking the difference $\Delta Q_{G} / \Delta V_{G 1}$, where $i=G, D, S$ and $B$. By using polynomial fitting to $Q_{G}$ data, it was hoped that a truer indication of capacitance properties could be obtained.

Figures 4.1(b), 4.2(b) and 4.3(b) show $C_{G G} \sim V_{G S}$ for the LD, MD and SD respectively, where original and smoothed curves are compared. The normalized caparitance characteristics of each device after smoothing are plotted for three drain bias condition in Figures 4.4-4.13. As can be seen, only parts of some curves need smoothing; i.e. all gate capacitance curves at $V_{D S}=0.1 \mathrm{~V}$ and all the curves in the subthreshold and part of the saturation region, somewhat above threshold, are sufficiently smooth in their original form. It may also be noted from the original characteristics extracted from MINIMOS, plotted in normalized form in Figures $4.14-4.22^{2}$, that parts of certain curves exhibit so much numerical scatter that it

2 In order to facilitate the discussion of short channel effects in Sections 4.2 -4.5, each 
is not possible to represent their true behavior using polynomials from the few and scattered $Q_{G}$ data points available, as for example, the case of $C_{G G} \sim V_{G S}$ for the $L D$ at $V_{D S}=4.0 \mathrm{~V}$. In such a case the estimated best curve was fit ted by hand to the capacitance behavior expected for the particular region from known characteristics of large devices; such estimated behavior is indicated by dotted lines throughout Figures $4.4-4.13$, whereas the dashed lines represent capacitance characteristics evaluated from smoothed $Q_{G} \sim V_{G S}$ characteristics using polynomials; solid lines are used where no smoothing was necessary.

Different order polynomials, (ranging from second to sixth order, depending upon circumstances), were found by the method of least squares. In order to improve accuracy, some gate charge curves had to be divided into sections and treated separately using different polynomials which fit each range more reasonably. The breaks shown on these curves mark the different ranges; this sectioning was particularly necessary for the case of $C_{G G}$, where the characteristics undergo many changes of form and more generally, for the characteristics of the LD, which exhibited more scatter, for the reasons discussed in the previous section. In certain cases, several data points are clearly erroneous, producing a significant increase in scatter locally, interfering with the use of a polynomial to show the true behavior; for example, $C_{G D}$ for the SD at $V_{D S}=2.0 \mathrm{~V}$ and $V_{D S}=4.0 \mathrm{~V}$ and $5.0 \mathrm{~V} \leq V_{G S} \leq 6.2 \mathrm{~V}$. In these situations, it was often possible to find suitable polynomials by simply skipping the few obviously erroneous data points.

In order to be able to smooth the $Q_{G}$ data in the presence of large scatter, either by the use of polynomial fitting to the data points or by hand estimate, it was necessary first to have a good understanding of the behavior of each of the gate capacitances, so that important features, i.e. such as due to short channel efferts (SCE), for example, do not get lost in smoothing process. The most difficult curves to treat were those of the LD. Long channel theory was often uied as a guide to

of the figures, which are shown at the end of this chapter, presents data for the I.D, MD and SD for a particular value of drain voltage. 
help reveal the expected features of the curves which were hidden in the numerical scatter.

An example of the approach taken for smoothing is given for $C_{G G}$ of the LD at $V_{D S}=4.0 \mathrm{~V}$, which is also the curve with the most scatter. The capacitance characteristics obtained from MINIMOS are shown in Figure 4.1(b) with the smoothed results superimposed. Seeing that the curve is reasonably smooth up to $V_{G S} \simeq 1.2 \mathrm{~V}$, this region is left in its original form. However, for the remainder of the voltage range shown, it is not possible to deduce the capacitance behavior in any definitive way owing to the extent of the numerical scatter of data present, particularly for the region $1.2 \mathrm{~V} \leq V_{G S} \leq 5.2 \mathrm{~V}$. In this case, where the true behavior of the curve is masked and polynomial fitting to the available data is ineffective, a handdrawn estimate of the curve is made, based on existing knowledge of long channel characteristics. The curve is expected to remain flat, with a slight reduction of $C_{G G}$ and increasing $V_{G S}$, throughout the saturation region and to begin rising sharply towards $C_{0}$ (at a rate similar to the curve at $V_{D S}=2.0 \mathrm{~V}$ ), at the saturation/liv $\mathrm{r}$ boundary. According to LCT, at $V_{\text {gsat }}=6.0 \mathrm{~V}, C_{G G}=1.1 \mathrm{pF}$ or $0.65 \mathrm{C}_{\text {。 }}$ and is used as a guide to the shape of the curve. The reduced scatter in the range $5.2 \mathrm{~V} \leq V_{G S} \leq 7.0 \mathrm{~V}$ reveals the expected behavior in $C_{G G}$; however, the increase apprars to occur prematurely at $V_{G S}=5.2 \mathrm{~V}$. The reduction in scatter permits the use of a polynomial for smoothing in this range, but the accuracy of the curve is certainly in question.

The amount of scatter of data and the difficulty of applying polynomial smoothing, are reduced as the channel length is made shorter. Hence, polynomial fitting was possible for all the curves of the $\mathrm{SD}$, with the exception of the small region at the end of the curves, where the scatter of $Q_{G}$ data points is large around the initial simulation conditions.

\subsection{Gate Self-Capacitance $C_{G G}$}

Here and in Sections $4.3-4.5$ the properties exhibited by the results due to short channel effects are discussed, whereas previously the co.acern was with accuracy of 
simulation. The study of SCE is facilitated by presenting the original and smoothed capacitance data for $C_{G G}$ (and later $C_{G D}, C_{G S}, C_{G B}$ ) $\sim V_{G S}$ in normalized form with respect to $C_{o}$ in Figures $4.14-4.22$ and $4.4-4.13$ respectively.

It is seen that due to the influence of effects associated with the $2 \mathrm{D}$ electric field regions near the source and drain certain expected changes are noted, the first being the threshold voltage reduction for the short channel devices compared with the long channel devices. This corresponds to a shift of the capacitance characteristics which, though small, is noticeable in Figures $4.14-4.16 ; C_{G G}$ of the SD is shown to begin rising at a lower gate voltage than the LD.

Figures $4.4-4.6$ and $4.14-4.16$ also show rounding off and spreading out of the capacitance characteristics with channel length reduction; i.e. $C_{G G}$ rises more steeply for the LD around threshold, than it does for the SD. When threshold is reached, essentially the whole length of the channel becomes inverted (except for the relatively small area near the drain which is pinched off in saturation), whereas in the SD the drain space charge region occupies a significant fraction of the distance between source and drain and the reduced channel slowly stretches towards the drain as $V_{G S}$ increases, thus corresponding to a gradual increase in the component $C_{G S}$ (which is discussed in more detail later); this effect is mainly responsible for the gradual increase in $C_{G G}$.

In the LD the saturation point is well defined and can be identified by the sharp change in the $C_{G G} \sim V_{G S}$ characteristics, as can be seen from the original and smoothed characteristics of Figures 4.1(b) and 4.4. In the SD however, the pinch-off point modulates throughout the length of the channel for varying biases and furthermore, the capacitance values remain small (due to the reduced devire area), therehy making the saturation point less distinct.

The SCE discussed so far are well known[4,14,16,19] and were expected to be present in the short channel characteristics from MINIMOS; however, other important short channel effects were observed on the gate capacitances. The most obvions is the $C_{G G}$ dependence on $V_{D S}$ in the subthreshold region. From the original char- 
acteristics of Figures $4.14-4.16$, it is clear that the capacitances become separated, due to a reduction of $C_{G G}$ with increasing $V_{D S}$, as the channel length is reduced. It is believed that the main reason for this property is that in the subthreshold region $C_{G G}$ is essentially the MOS capacitance $C_{G B}$ between the gate and bulk, the effective gate area of which is increasingly sensitive to the modulation of the width of the drain space charge region by $V_{D S}$. Thus, as $V_{D S}$ is increased in the SD, the gate controlled region inside the channel is reduced, corresponding to a decrease in the component $C_{G B}$ and therefore in $C_{G G}$.

\subsection{Capacitance Component $C_{G D}$}

Although the MINIMOS manual suggests a maximum voltage range of 5 volts while holding the grid fixed between simulations, it was found that the range $1.0 \mathrm{~V} \leq$ $V_{G i} \leq 7.0 \mathrm{~V}$ was permissible; this was expected to be a sufficient range to investigate the changes in the behavior of the short channel characteristics, $C_{G D} \sim V_{G S}$.

Upon first glance, the characteristics shown in Figure 4.17 appear to suggest an increase in $C_{G D} / C_{o}$ for a reduction in $L_{e f f}$, i.e. due to SCE on $C_{G D}$; however, this is doubtful for two very important reasons: 1) with (essentially) no drain hias applied i.e. $V_{D S}=0.15 \mathrm{~V}$, the characteristics for $C_{G D}$ (Figure 4.17) and $C_{G S}$ (Figure 4.20) should be identical, since the saturation region is reduced to a point as $l_{D S} \rightarrow 0$. From the figures however, it is clear that the curves are very different; 2) all the normalized curves in Figure 4.17 are well above $0.5 C_{0}$, the expected limit in the triode region, where $C_{G G}$ consists of the sum $C_{G D}+C_{G S}$, since $C_{G B}$ is issentially zero. Seeing that $C_{G S}$ is indeed close to $0.5 C_{0}$ for all device lengths in Figure 4.20, the sum $C_{G D}+C_{G S}$ is certainly greater than $C_{G G}$ over the whole range of $V_{G S}$ by about $10-15 \%$, depending on the device size. It is reasonable to conclude that the limited numerical accuracy of MINIMOS is responsible for these disagreements and thus the results for $C_{G D}$ are in error, whereas those for $C_{G S}$ are likely correct. A more detailed investigation of the simulation results for $C_{G D}$ below, appeared to confirm this conclusion. 
The value of $C_{G D}$ at $V_{G S}=6.0 \mathrm{~V}$ and $V_{D S}=0.15 \mathrm{~V}$ for the $\mathrm{LD}$, from Figure 4.17 is compared with $C_{G D}$ calculated using LCT in Table 4.7, where it is noted that the value of $C_{G D}$ from the curve is certainly overestimated by MINIMCS compared to $0.49 C_{0}$ predicted by theory; this doesn't prove however, that the relative increase of $C_{G D} / C_{o}$ for reduced channel lengths observed in Figure 4.17 is false.

\begin{tabular}{|c|c|c|c|}
\hline$C_{G D}$ & LD & MD & SD \\
\hline LCT & $-0.835 p F$ & - & - \\
& $0.49 C_{o}$ & & \\
\hline Separate Simulation & $-1.077 p F$ & $-49.96 f F$ & $-22.01 f F$ \\
& $0.63 C_{o}$ & $0.63 C_{o}$ & $0.64 C_{o}$ \\
\hline Figure 4.17 & $-0.939 p F$ & $-49.144 f F$ & $-21.64 f F$ \\
& $0.552 C_{o}$ & $0.62 C_{o}$ & $0.63 C_{o}$ \\
\hline
\end{tabular}

Table 4.7 Comparison of $C_{G D}$ values from Figure 4.17, with seperate simulations and LCT at $V_{G S}=6.0 \mathrm{~V}, V_{D S}=0.15 \mathrm{~V}$.

Table 4.7 also compares values for $C_{G D}$ from discrete simulations with those from the curves at $V_{G S}=6.0 \mathrm{~V}$ and $V_{D S}=0.15 \mathrm{~V}$. The results from the discrete simulations are consistent with those for GRIDFREEZE, with values of $C_{G D}$ greater than $0.5 C_{0}$ for the different devices ${ }^{3}$. Thus, regardless of how the simulations were made there is a significant error in the magnitude of $C_{G D}$ for $V_{D S}=0.15 \mathrm{~V}$. Therefore, it can safely be concluded that numerical computation errors in MINIMOS are responsible for the separation in the $C_{G D} \sim V_{G S}$ curves of $V_{D S}=0.15 V$ and not SCE. It appears that these results are wrong in scale, and that the best interpretation of them would be obtained if they were re-scaled to give $C_{G D} \rightarrow 0 . \Delta C_{\omega}$ for $V_{G S} \rightarrow \infty$.

3 This is not surprising according to the discussion on Section 4.1.1; discrete simulations are expected to give results at least as erroneous as those employing the option GRIDFREEZE set at larger initial bias conditions. 
The most noticeable SCE on the $C_{G D}$ characteristics is observed from Figure 4.17 for $V_{D S}=0.15 \mathrm{~V}$ which, though inaccurate in magnitude (as concluded above), predicts the expected behavior of the short channel $C_{G D} \sim V_{G S}$; the knee of the curves is clearly shown to shift to the left as $L_{e f f}$ is reducer!, which was also discussed earlier in the analysis of $C_{G G}$. It should be noted however, that the amount of the shift is exaggerated from this figure (as compared to Figure 4.20 for $C_{G S}$ ) and that with rescaling as suggested above, reasonable agreement would be obtained.

Other changes in the characteristics due to SCE, such as spreading of the $C_{G D}$ curves can also be observed from Figures $4.18,4.19$. It can be seen that the saturation/linear boundary becomes more vague as $L_{\text {eff }}$ is reduced, as expected from the earlier analysis of $C_{G G}$.

Figures 4.18 and 4.19 show an increase in $C_{G D} / C_{o}$ in the saturation region of operation. In this range of $V_{G S}$ the separation between $C_{G D} / C_{o}$ for different $L_{e f f}$ is not significantly influenced by changes in drain voltage ${ }^{4}$. Table 4.8 compares the actual values of $C_{G D}$ at $V_{G S}=1.0 \mathrm{~V}$.

\begin{tabular}{|c|c|c|}
\hline$V_{D S}(V)$ & $\mathrm{MD}$ & $\mathrm{SD}$ \\
\hline 2.0 & $3.29 f F$ & $4.45 f F$ \\
4.0 & $4.51 f F$ & $4.01 f F$ \\
\hline
\end{tabular}

Table $4.8 C_{G D} ; V_{G S}=1.0 \mathrm{~V}$.

It can be noted that the actual values of $C_{G D}$ are approximately the same order of magnitude for the given drain voltages and do not tend to increase for shorter device. But these values become more dominant for smaller devices, in which the gate oxide capacitance $C_{o}$ is small. Thus, the $C_{G D} / C_{o}$ values increase as $L_{\text {eff }}$ is reduced.

\footnotetext{
4 IDS dependence of $C_{G D}$ for small devices have been predicted[21] and will be discussed later, but any differenices in the separation of $C_{G D} / C_{o}$ for different $L_{e f f}$ and $V_{D S}$ were undetectable, given the degree of scatter present from MINIMOS simulation.
} 
The increase in $C_{G D} / C_{0}$ for the SD in saturation can be explained by the proportionately increased influence of the weak inner fringing capacitance component $C_{G D(i f)}$. This acts as a small coupling capacitance between the gate and drain due to the effective sepration of the drain from the inverted channel. This small but significant capacitance component is also responsible for the more gradual change of $C_{G D}$ in the transition from saturation to triode operation in the short channel case as compared with the LD, because the capacitances remain small for the SD.

The separation between the curves of the various devices due to the increased influence of $C_{G i D(i f)}$, shown in Figure 4.18 and 4.19 , continues until the saturaticn/linear boundary is reached when the inverted channel joins with the drain of the device and $C_{G D}$ begins to rise.

It can $x$ concluded, therefore, that $C_{G D(i f)}$ becomes significant in short channel device characteristics and cannot be ignored as in the case of LD.

\subsection{Capacitance Component $C_{G S}$}

Figure 4.20 shows the normalized $C_{G S} \sim V_{G S}$ characteristics for the three devices, at $V_{D S}=0.15 \mathrm{~V}$. Once saturation sets in, the three curves begin to overlap, as expected. In order to eliminate any doubt about the predictions of $C_{G S} \sim V_{G S}$ made by MINIMOS, independent simulations were made for the $\mathrm{SD}^{5}$ at $V_{G S}=6.0$ ? and $V_{D S}=0.15 \mathrm{~V} . C_{G S}$ was evaluated to be $-16.725 f F$ or $0.484 C_{o}$, in very good agreement with the curve. Hence, there is no doubt that Figure 4.20 gives a more accurate representation of $C_{G S} \sim V_{G S}$ than that of $C_{G D} \sim V_{G S}$ from Figure 4.17, which is decidedly in error.

The smoothed characteristics shown in Figures $4.4-4.13$ and the original normalized results from Figures $4.14-4.22$ show the expected SCE, such as rounding of the curves and a less distinct threshold point with reduced $L_{\mathrm{eff}}$.

\footnotetext{
5 If the vertical shifting of the $C_{G D} \sim V_{G S}$ characteristics shown in Figure 4.17 were correct predictions caused by $S C E$, then $C_{G S} \sim V_{G S}$ for the SD should show the largest changes due to the same effect in such a way, as to satisfy the relationship $\left(C_{C_{i}} / C_{0}\right)+$ $\left(C_{G S}{ }^{\prime} C_{o}\right)=\left(C_{G G} / C_{o}\right), C_{G B}$ being essentially zerc above $V_{T H}$ for small devices.
} 

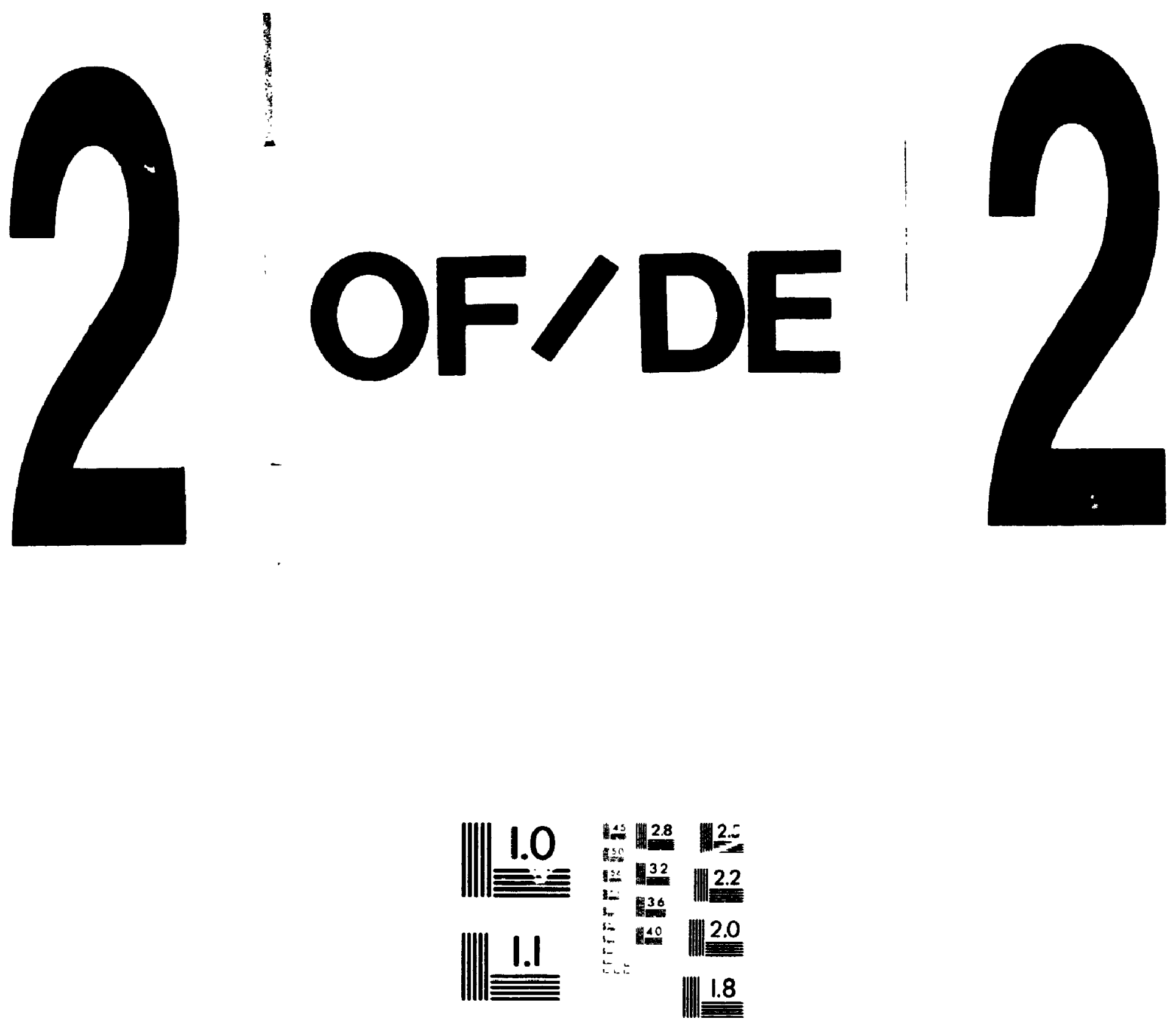

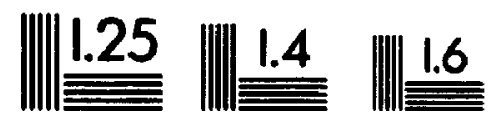

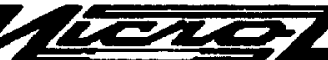


An important change in $\left(C_{G S} / C_{0}\right) \sim V_{G S}$ due to SCE is the increase in $C_{G S} / C_{0}$ with reduced $L_{\text {eff }}$ in the subthreshold region, shown in Figures $4.20-4.22$. Once again this apparent increase for small devices is due to the increased importance of the inner fringing capacitance component $C_{G S(i f)}$ on the short channel characteristics. This gradual rise in $C_{G S} / C_{0}$ for the $\mathrm{SD}$ in the subthreshold region is well represented for all three sets of normalized curves.

Beyond threshold $\left(C_{G S} / C_{0}\right) \sim V_{G S}$ do not show any noticeable changes in the curves due to channel shortening. With the exception of the few points at the end of each curve, which should be disregarded as numerical scatter (as previously discussed in Section 4.1.1), the curves of the SD are the most well behaved, with those of the LD being the most erroneous, in particular at $V_{D S}=4.0 \mathrm{~V}$, where a huge spike is observed at nearly twice the value of $\sim 2 / 3 C_{o}$ expected for $C_{G S}$ in the saturation regime.

It can be concluded therefore, that the SCE that can be observed in the $C_{G S} \sim$ $V_{G S}$ characteristics of Figures $4.20-4.22$ are the more gradual rise of $C_{G S}$ with $V_{G S}$ for the shorter devices and the increase in the normalized capacitances in the subthreshold region, due to the increased influence of $C_{G S(i f)}$, which as in the case of $C_{G D}$, cannot be ignored for short channel devices. It was however, impossible to observe any increase in the vagueness of the saturation/triode boundaries from these curves because of the overwhelming presence of scatter in these regions.

\subsection{Capacitance Component $C_{G B}$}

The $C_{G B} \sim V_{G S}$ characteristics were extracted only for the MD, so completing the set of gate capacitance components derived from MINIMOS for this device. These characteristics proved to be very valuable because $C_{G B}$ was found to be sensitive to drain voltage changes in short devices, shown in Figure 4.13, the behavior of which is reflected in $C_{G G}$, where the responsible mechanisms have been discussed.

$C_{G B}$ evaluated from independent simulations made for the LD in the subthreshold $\left(V_{G S}=0.4 \mathrm{~V}\right)$ and for three values of $V_{D S}$ are listed in Table 4.9; the results 
show that $C_{G B}$ is not a function of drain voltage in subthreshold for the LD, as expected. The small fluctuations in $C_{G B} / C_{o}$ are due to numerical computation errors in MINIMOS.

\begin{tabular}{|c|c|c|c|}
\hline$V_{D S}(V)$ & 0.1 & 2.0 & 4.0 \\
\hline$C_{G B} / C_{\diamond}$ & 0.39 & 0.40 & 0.38 \\
\hline
\end{tabular}

Table 4.9 Normalized $C_{G B}$ values obtained from independent simulations for $L_{\text {eff }}=$ $49.3 \mu \mathrm{m} ; \quad V_{G S}=0.4 \mathrm{~V}$.

\subsection{Concluding Remarks}

Based on the results of the gate capacitances obtained from MINIMOS, it can be concluded that the numerical scatter observed in the characteristics is due to the automatic generation of the mesh pattern, which is not always best suited to the simulation requirements for capacitance studies. In general, the amount of numerical scatter was found to increase with $L_{\text {eff }}$ and/or bias voltage, especially around the saturation point, where large potential gradients in the device are probably not properly represented. There are problems of calibration (i.e. the failure to predict $C_{G S} \rightarrow C_{G D} \rightarrow 0.5 C_{o}$ for $\left.V_{G S} \rightarrow \infty\right)$ and inexplicable inaccuracies such as the vertical separation in $\left(C_{G D} / C_{0}\right) \sim V_{G S}$ for $V_{D S}=0.15 \mathrm{~V}$, shown in Figure 4.17 and the closeness of the $C_{G G} \sim V_{G S}$ curves of the SD, at $V_{D S}=2.0 \mathrm{~V}$ and $4.0 \mathrm{~V}$.

Being able to choose the grid pattern has proved beneficial in extracting gate caparitances using 2D numerical simulation; see Ko[21], Dutton[16], Yeow[19], Chung [14]; in particular, it has been demonstrated recently by Chung[14] that the numerical scatter in the gate capacitance characteristics from MINIMOS simulation can be essentially eliminated by choosing the grid carefully, thereby rendering the process of smoothing the curves unnecessary. In the present work, smoothing of $Q_{G}$ data using polynomials was found useful as a means of estimating the capacitance behavior, particularly for curves with little scatter; for the cases where the numerical uncertainty was very large (i.e. for the LD), smoothing was estimated by hand, based on LCT and knowledge of long channel characteristics. In either case, the 
accuracy of the resulting plots is not very certain, even for curves which appear to have little scatter because, as mentioned above, there appeared to be some problems of calibration.

The effects of channel length reduction on the gate capacitances, shown best by the normalized capacitance curves of Figures $4.14-4.22$, were found to cause several changes to the $C_{G i} \sim V_{G S}$ characteristics that have been previously reported from measurement[16,19-21] and $2 \mathrm{D}$ simulation[14,16,19]; these are: 1) a shift of the characteristics to the left with channel length reduction; 2) rounding off of the gate capacitances as they tend to spread out for short devices, thus making operating boundaries such as $V_{T H}$ and $V_{\text {geat }}$ less distinct; 3 ) inner fringing capacitance components $C_{G D(i f)}$ and $C_{G S(i f)}$ become important in short devices and cannot be ignored in short channel capacitance models intended for CAD applications. The relative increase of $\left(C_{G D} / C_{0}\right)$ and $\left(C_{G S} / C_{0}\right) \sim V_{G S}$ in the subthreshold and saturaijon regions are due to the increased influence of these capacitance components, which are also partly responsible for the rounded off appearance of the $C_{G i} \sim V_{G S}$ curves.

It has also been shown for the first time (in Figure 4.13) that the MOS capacitance $C_{G B}$ (and hence, $C_{G G}$ ) is clearly dependent on $V_{D S}$ in the subthreshold region, this being due to the modulation of the size of the gate controlled channel region as $V_{D S}$ is varied in short channel devices.

According to results of measurement by Sheu and Ko[21], $C_{G D}$ and hence $C_{G S}$, are also dependent on $V_{D S}$ for $S C D$; that is, $C_{G D}$ would tend to increase with large drain voltages. This observation appears to make physical sense, since the amount of shielding of the inner fringing capacitance $C_{G D(i f)}$ by the surface mobile charge is reduced with increasing $V_{D S}$, as the inverted surface is pushed towards the source end, thereby increasing the component $C_{G D}$. Such an effect on $C_{G D}$ however, is not observed from the behavior of the curves in saturation in the present simulation work. Figures 4.18 and 4.19 show no noticeable increase in the separation of the curves during saturation between $V_{D S}=2.0 \mathrm{~V}$ and $V_{D S}=4.0 \mathrm{~V}$. This 
is in agreement however, with other reports of $C_{G D} \sim V_{D S}$ from 2D simulation by Dutton and al.[16] and Chung[14], which do not show any increase in $C_{G D}$ with increasing drain voltage either. It is thought that this discrepancy between simulation and measurement may be due to limitations in the representation of the mobility models used; it has been shown by Yeow[19] that realistic mobility models are necessary in order to obtain good agreement between simulation and measured gate capacitances. 


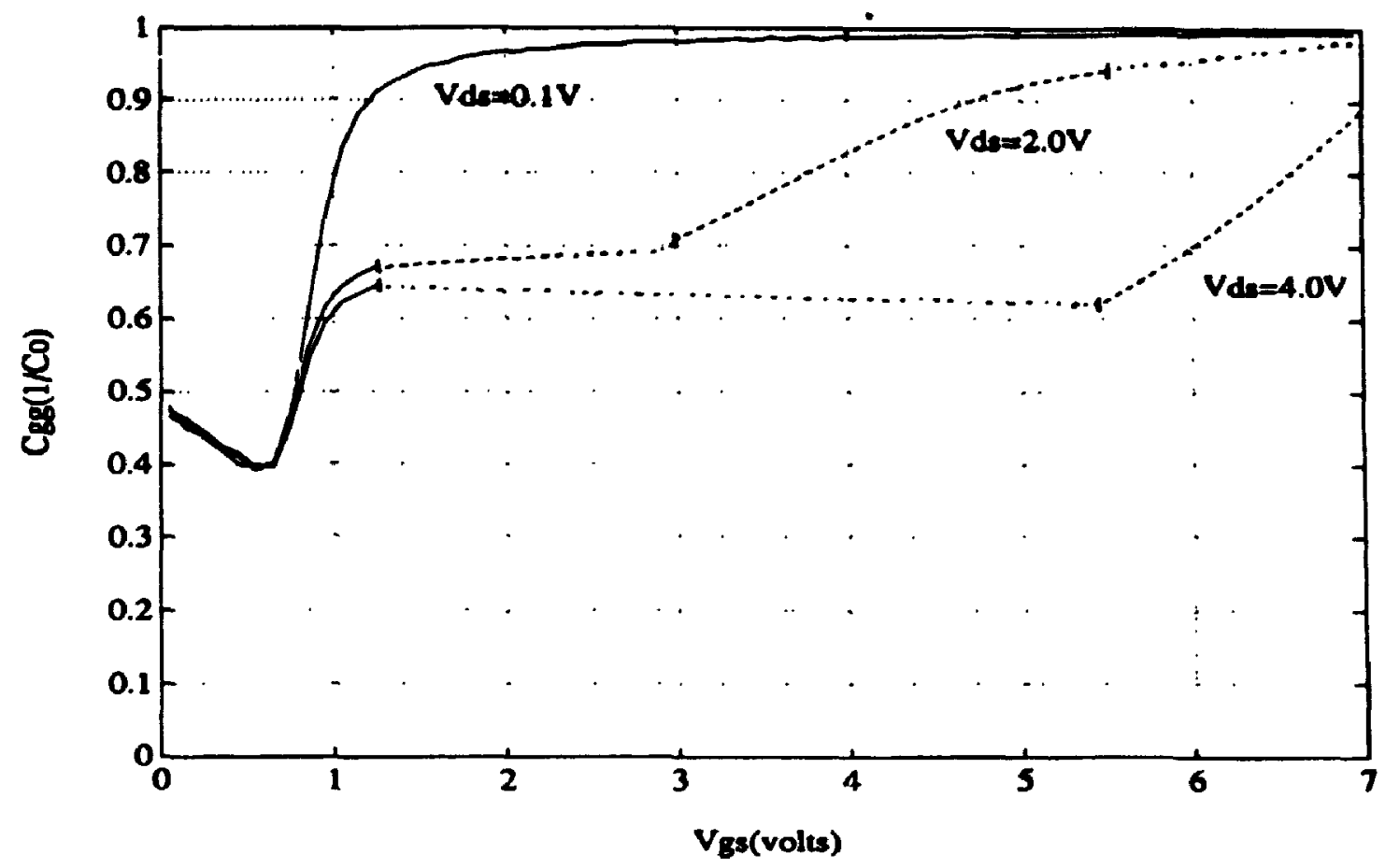

Figure 4.4 Smoothed normalized $C_{G G} ; L_{e f f}=49.3 \mu \mathrm{m}$.

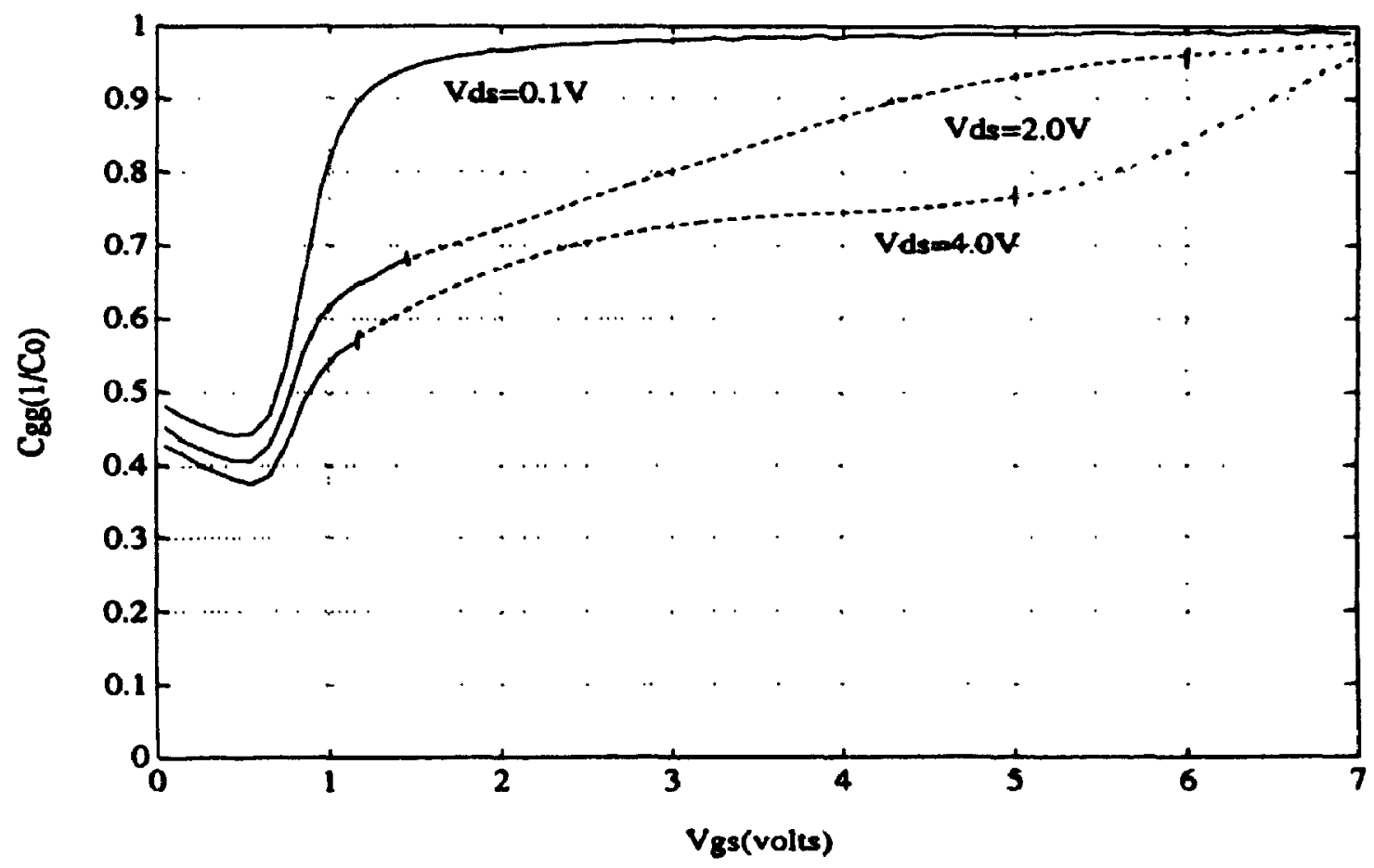

Figure 4.5 Smoothed normalized $C_{G G ;} L_{e f f}=2.3 \mu \mathrm{m}$. 


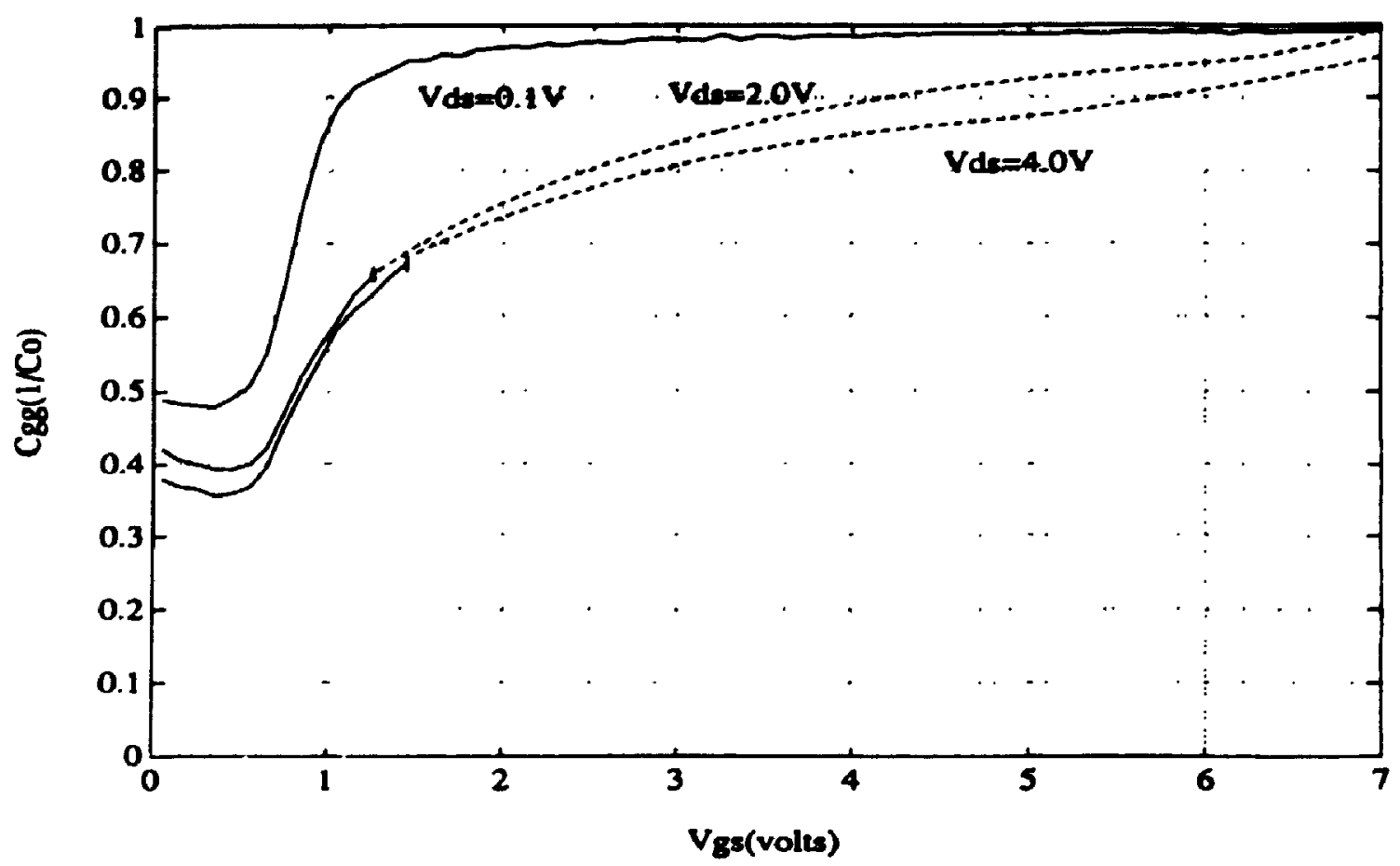

Figure 4.6 Smoothed normalized $C_{G G} ; L_{e f f}=1.0 \mu \mathrm{m}$.

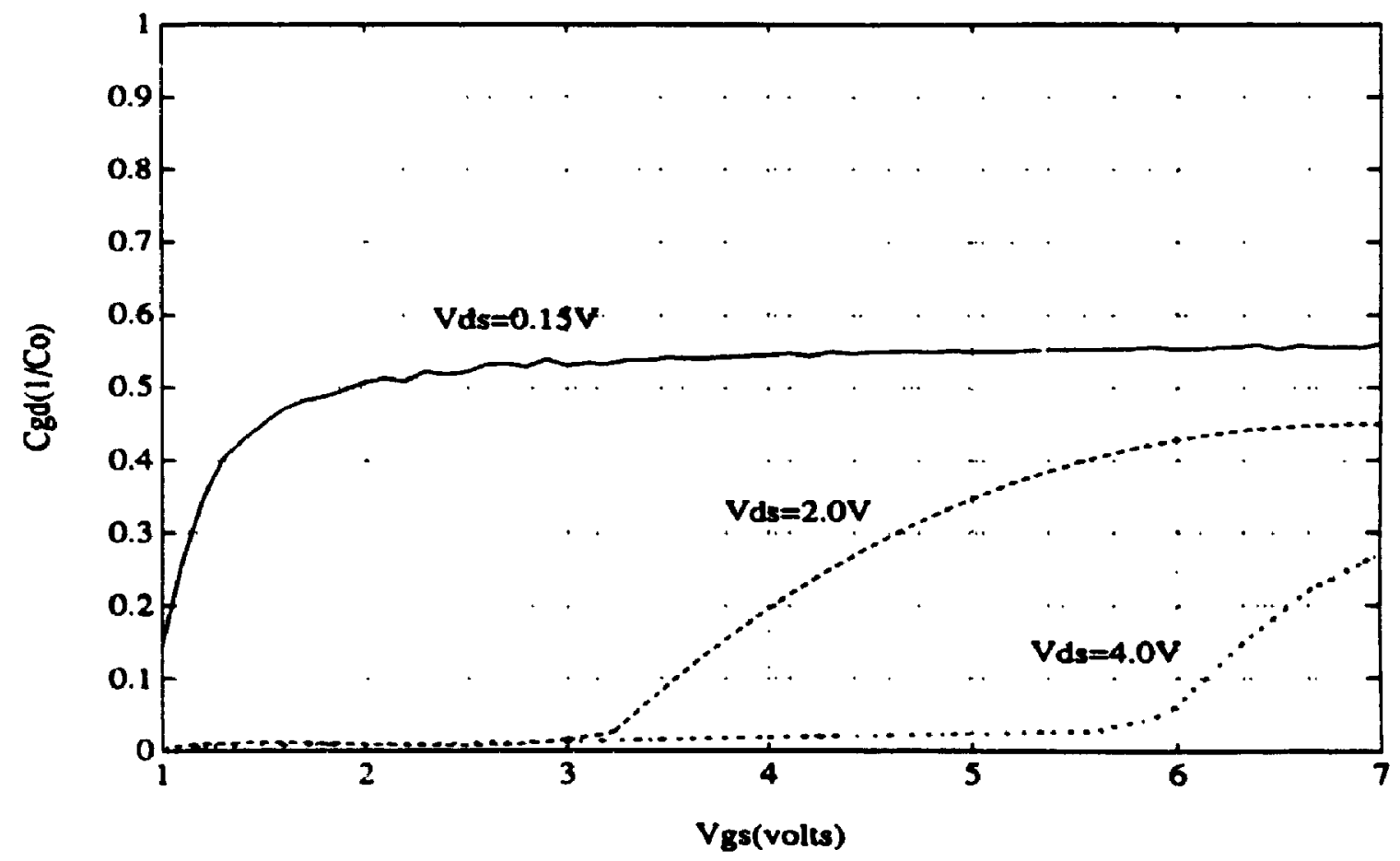

Figure 4.7 Smoothed normalized $C_{G D} ; L_{e f f}=49.3 \mu \mathrm{m}$. 


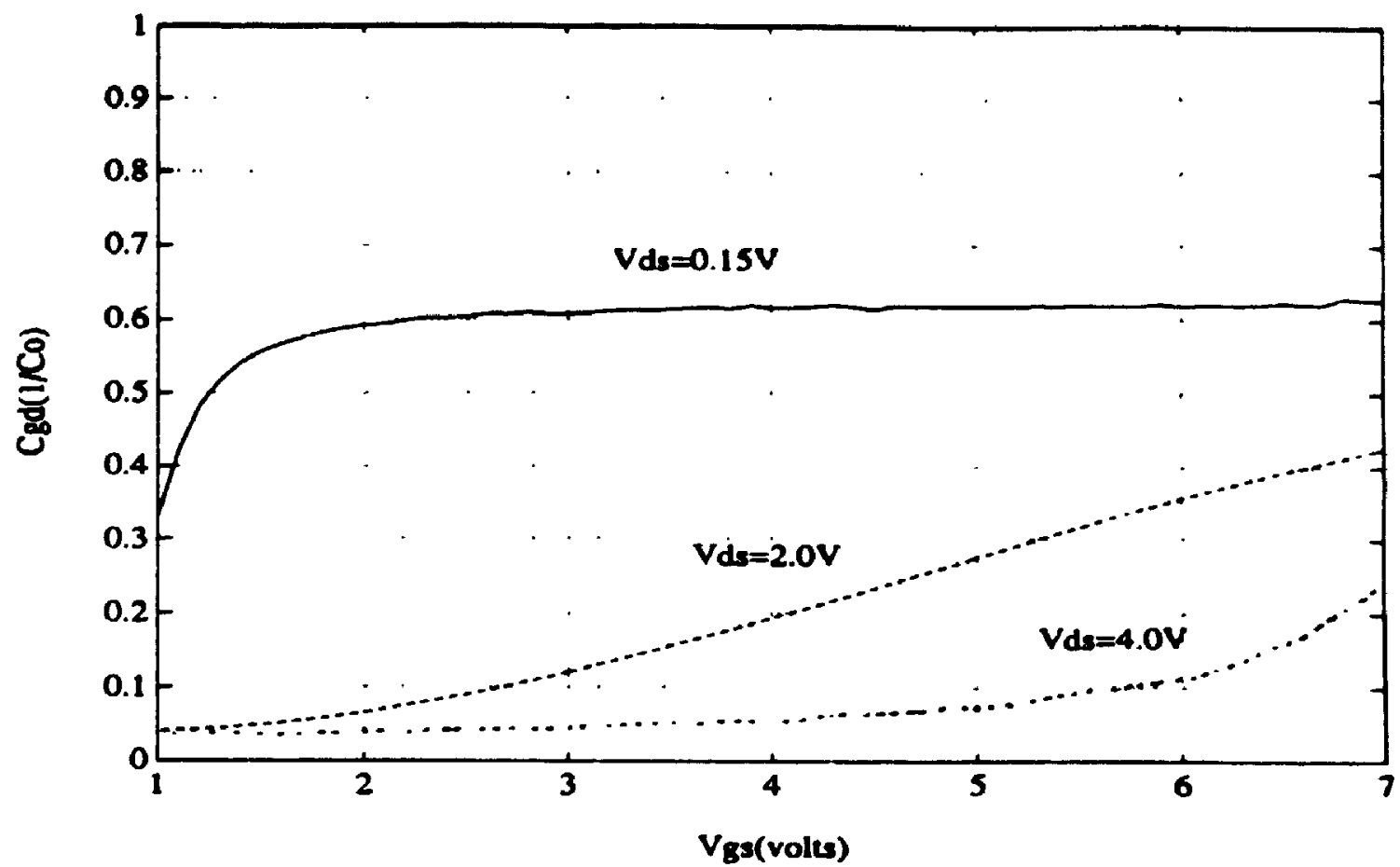

Figure 4.8 Smoothed normalized $C_{G D} ; L_{e f f}=2.3 \mu \mathrm{m}$.

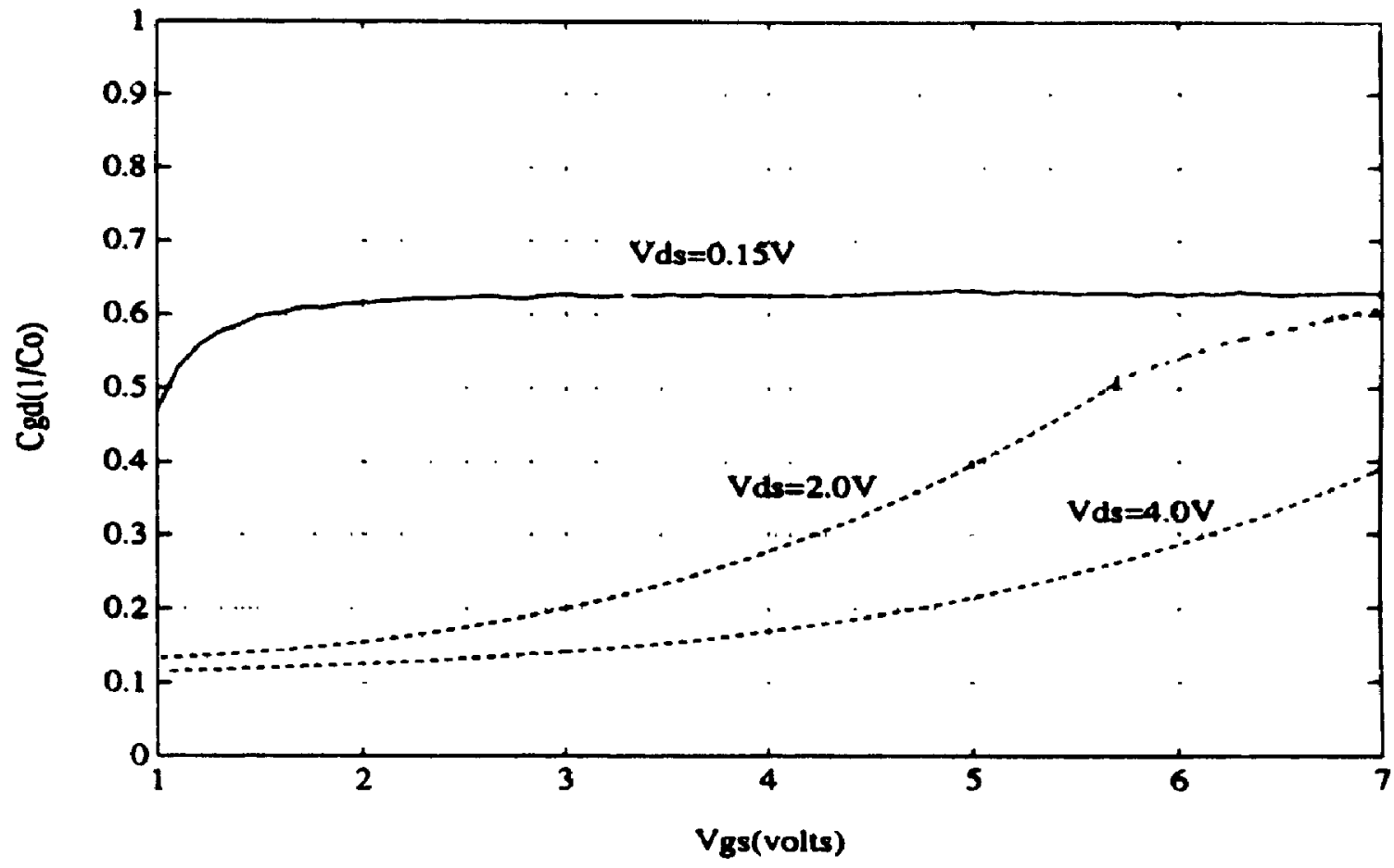

Figure 4.9 Smoothed normalized $C_{G D} ; L_{e f f}=1.0 \mu \mathrm{m}$. 


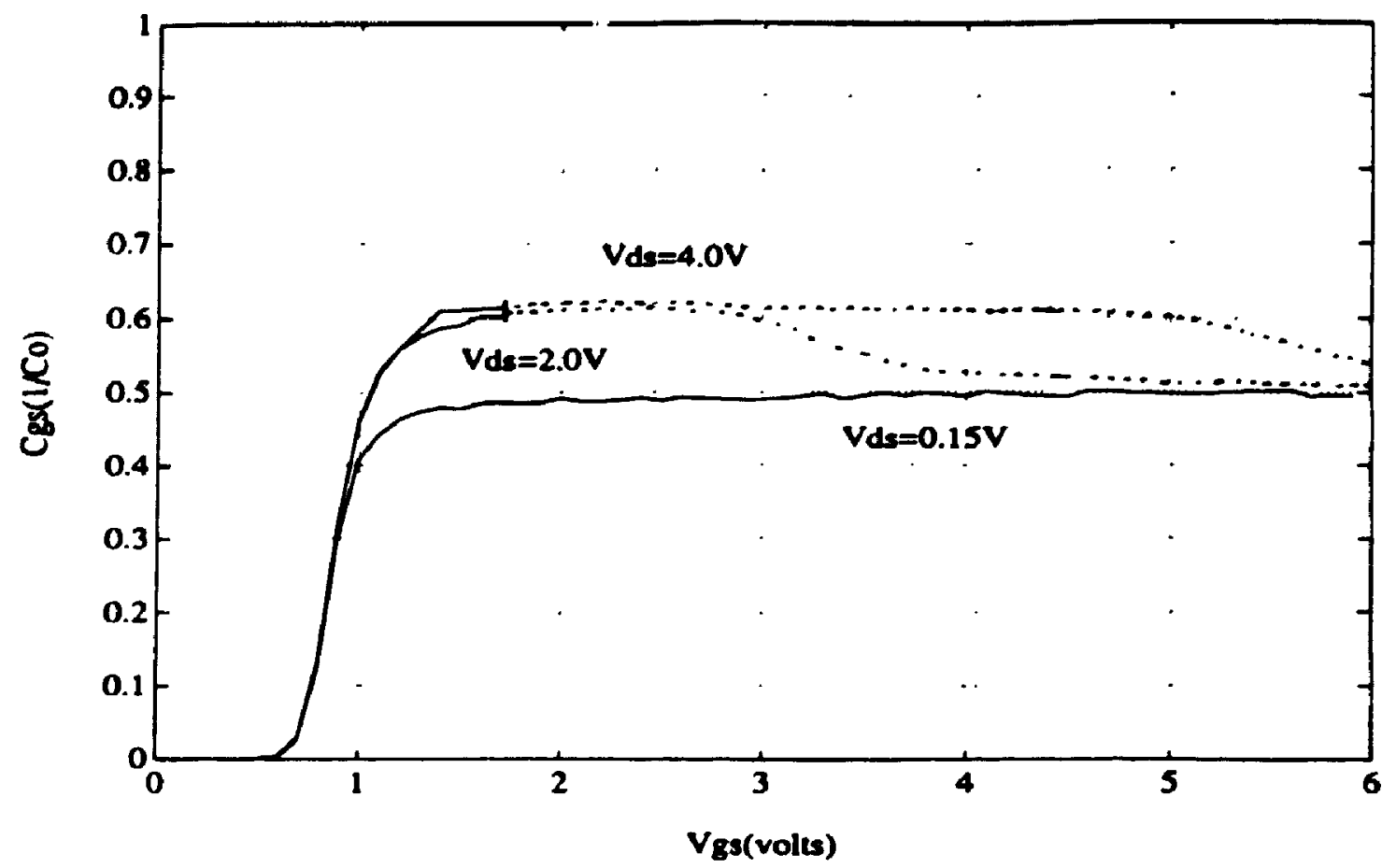

Figure 4.10 Smoothed normalized $C_{G S} ; L_{\text {eff }}=49.3 \mu \mathrm{m}$.

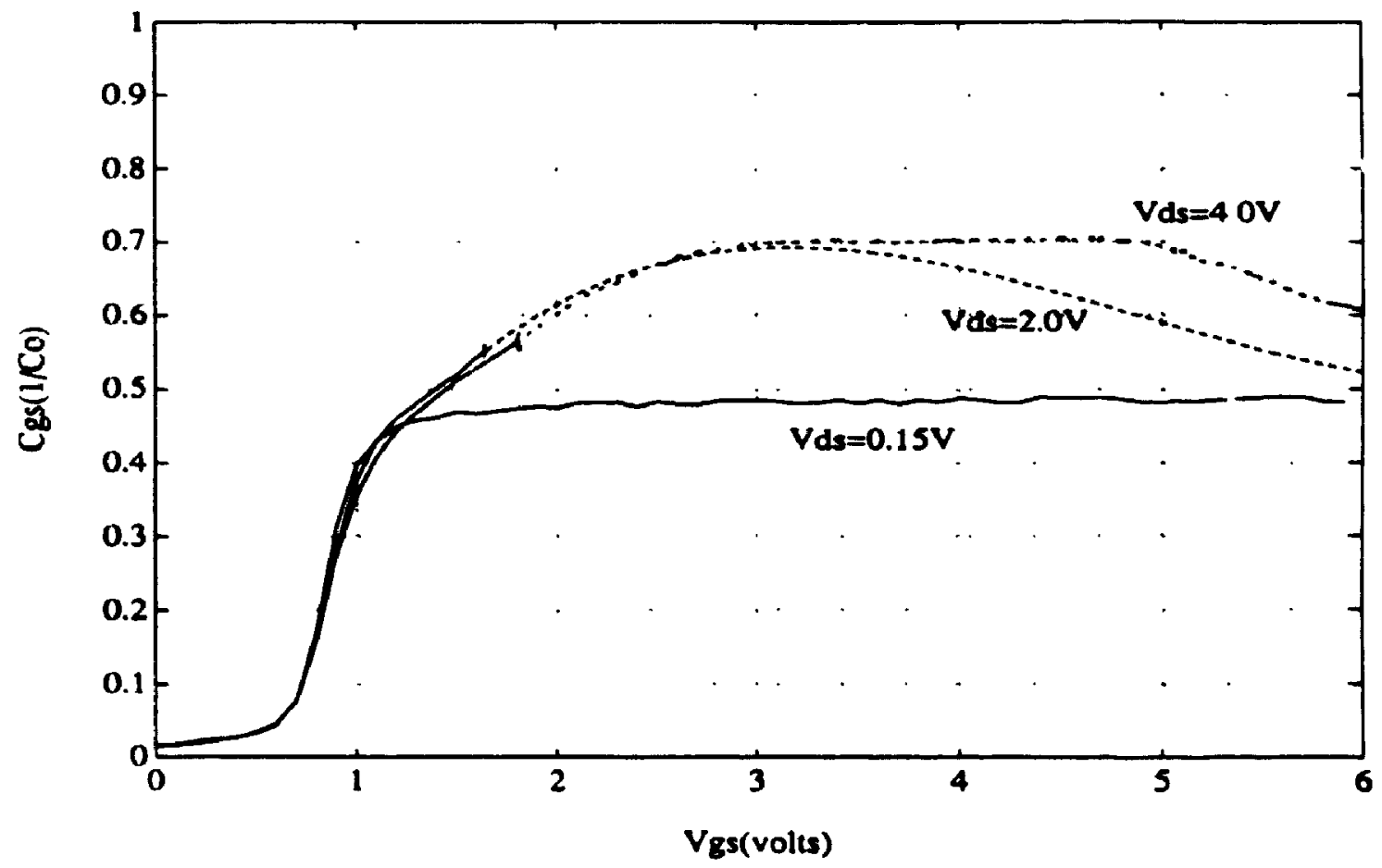

Figure 4.11 Smoothed normalized $C_{G S}: L_{e f f}=2.3 \mu \mathrm{m}$. 


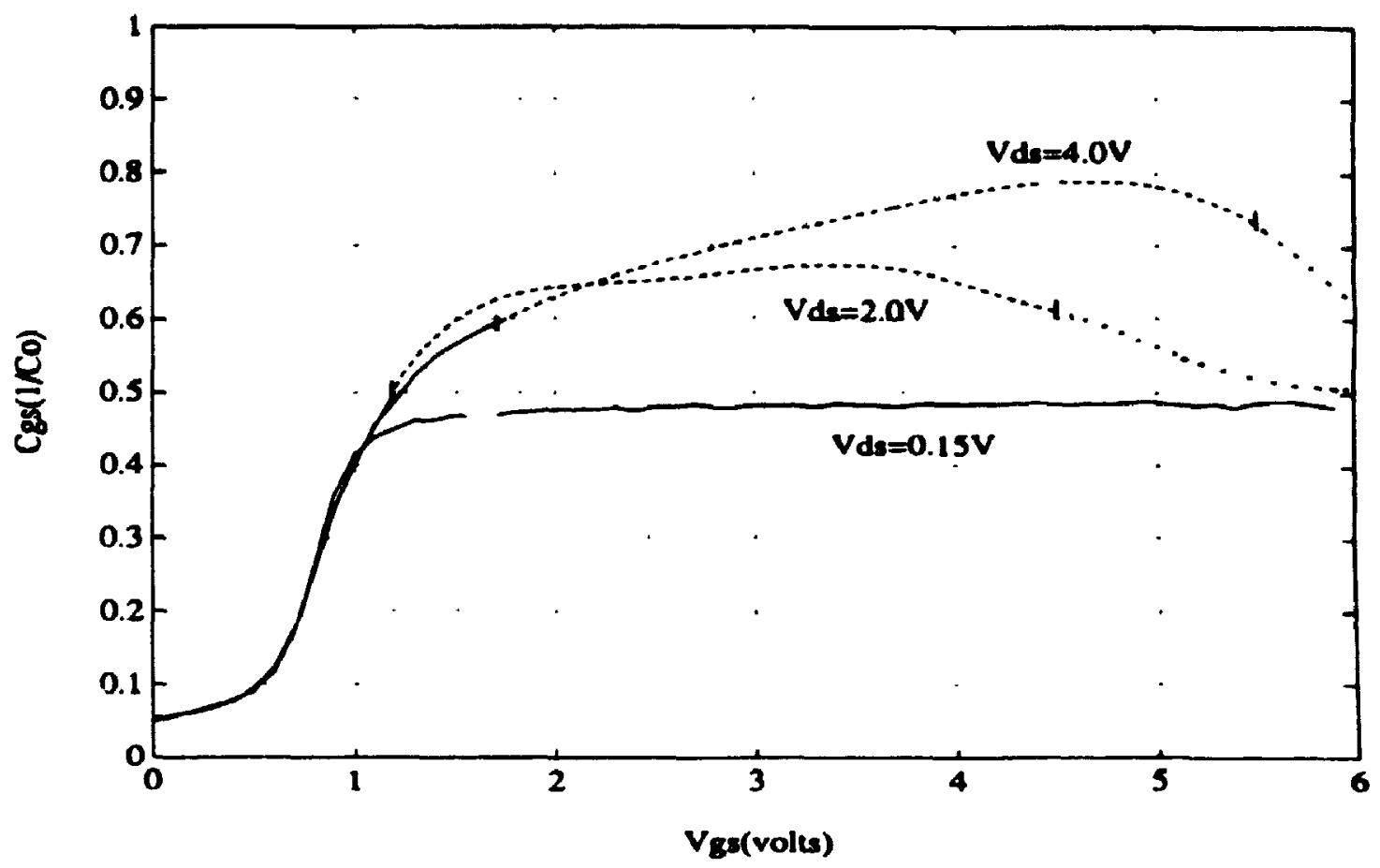

Figure 4.12 Smoothed normalized $C_{G S} ; L_{\varepsilon f f}=1.0 \mu \mathrm{m}$.

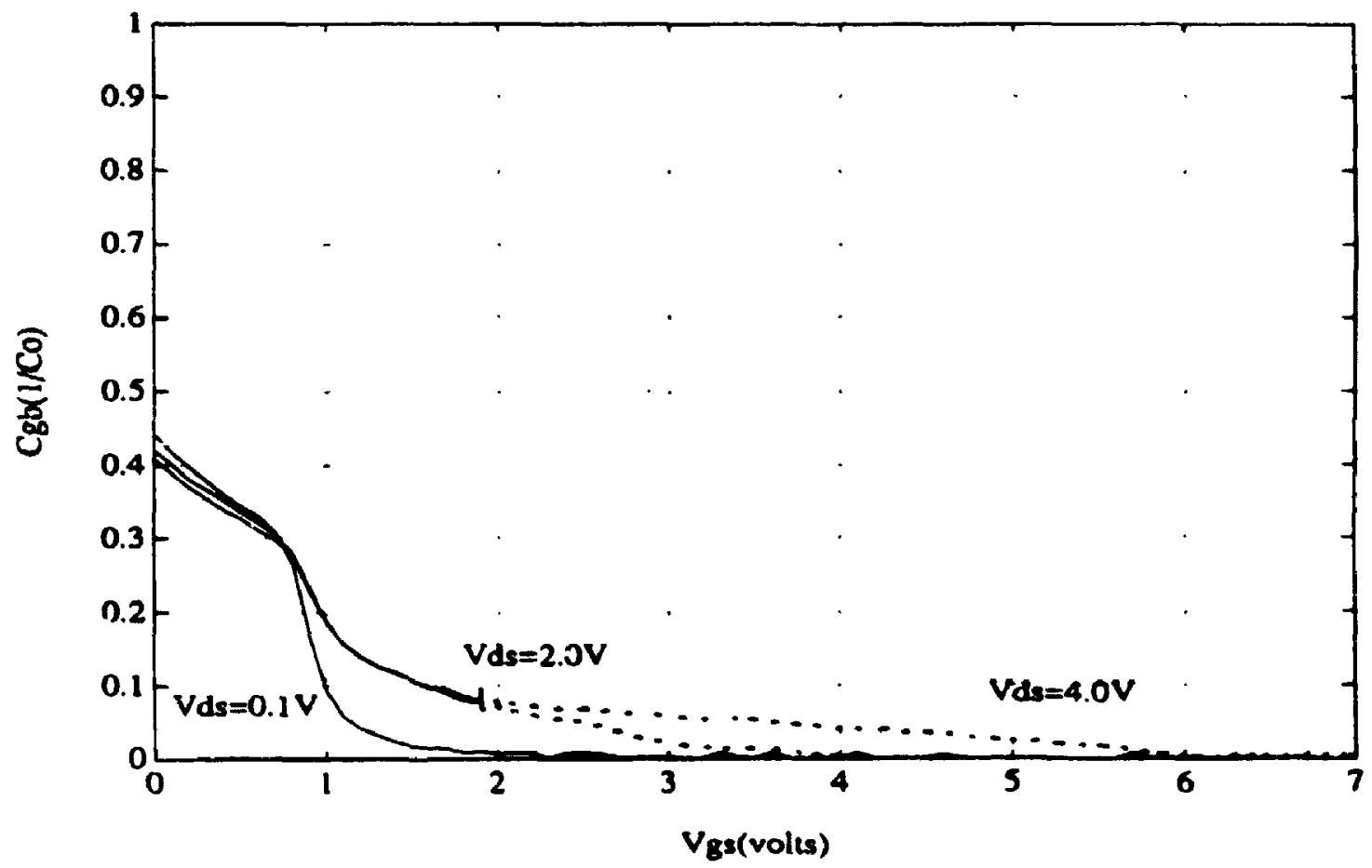

Figure 4.13 Smoothed normalized $C_{\sigma_{B} B} ; L_{\mathrm{e} J}=2.3 \mu \mathrm{m}$. 


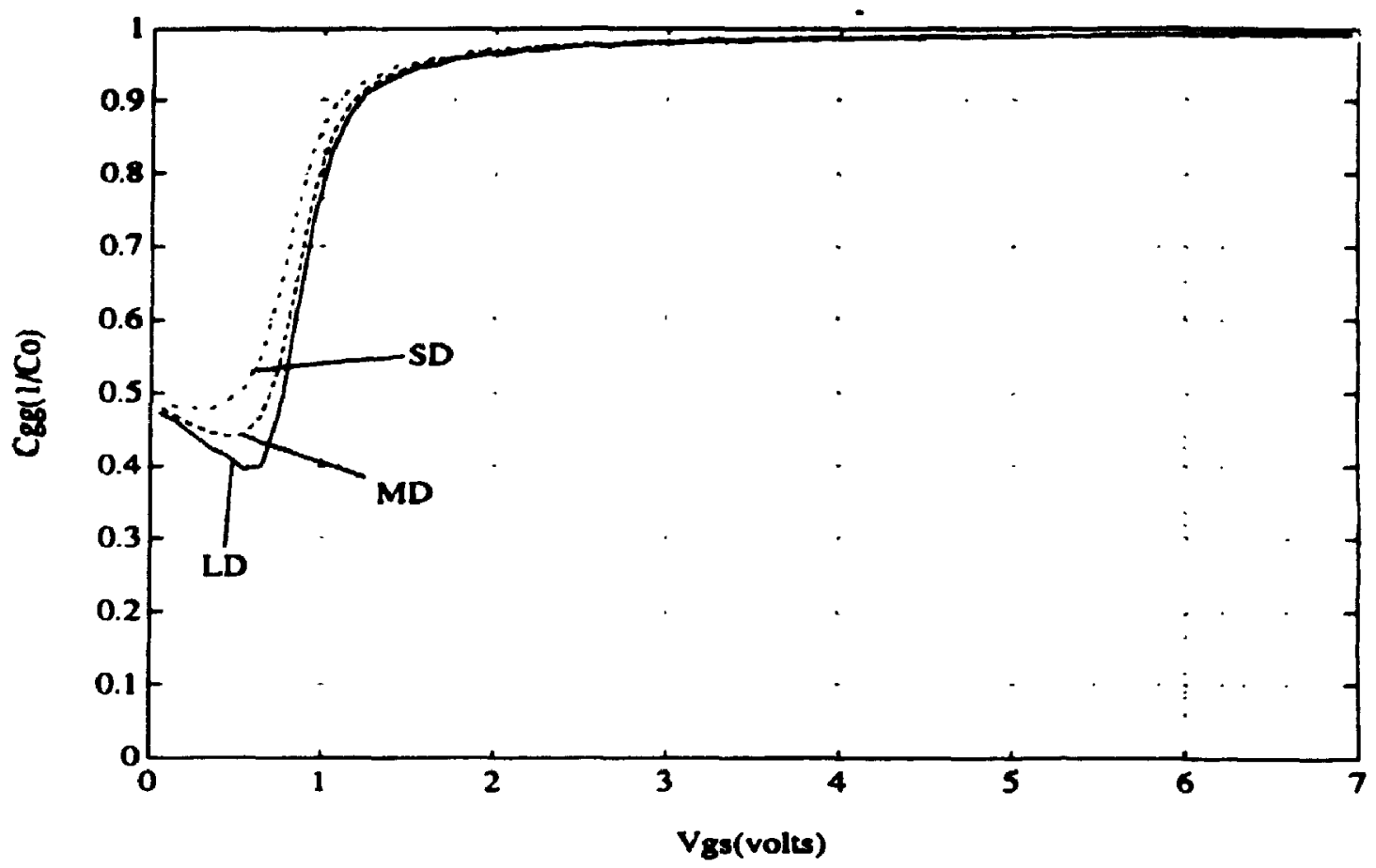

Figure 4.14 Normalized $C_{G G} ; V_{D S}=0.1 \mathrm{~V}$.

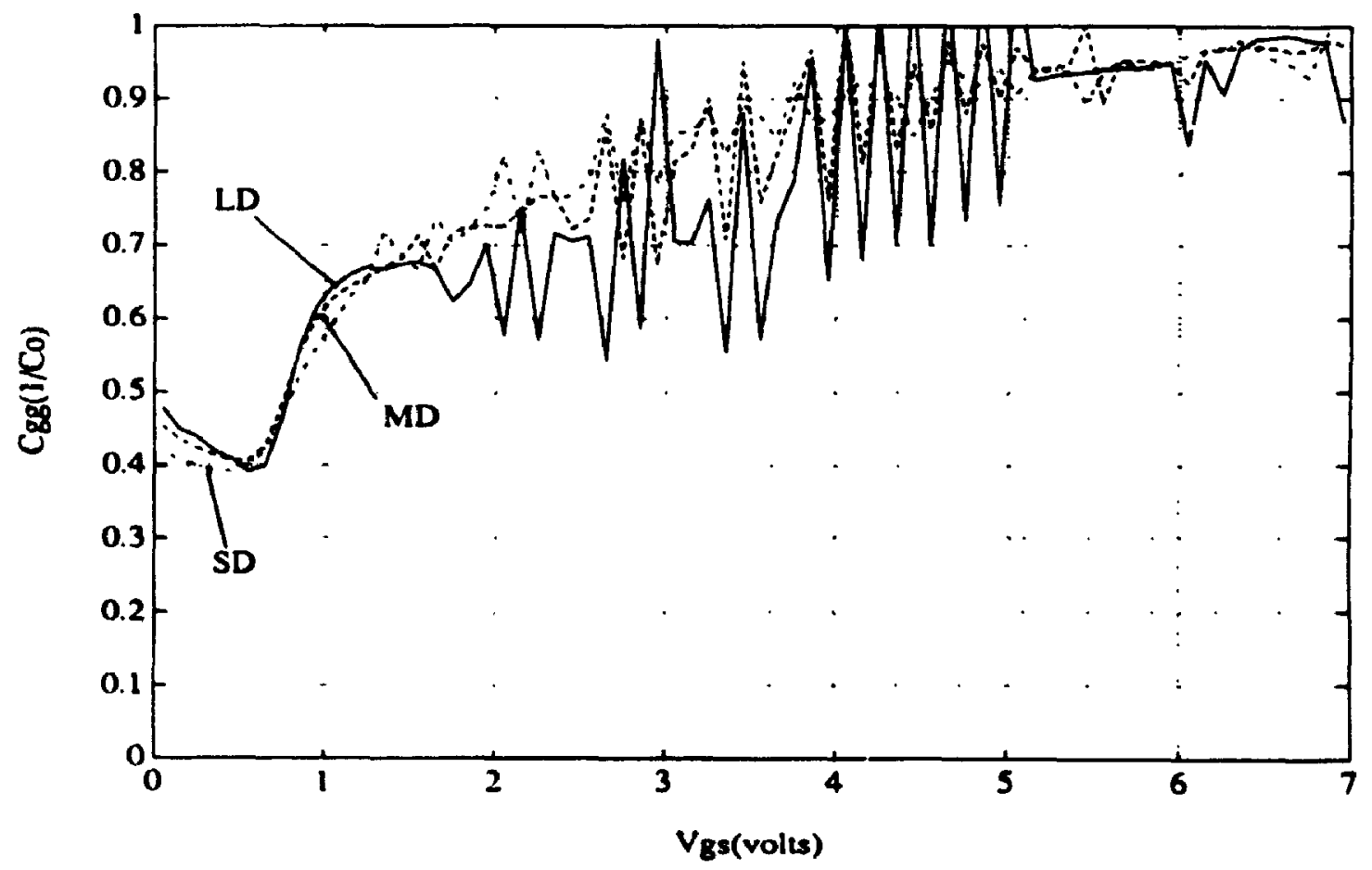

Figure 4.15 Norınalized $C_{G G} ; V_{D S}=2.0 \mathrm{~V}$. 


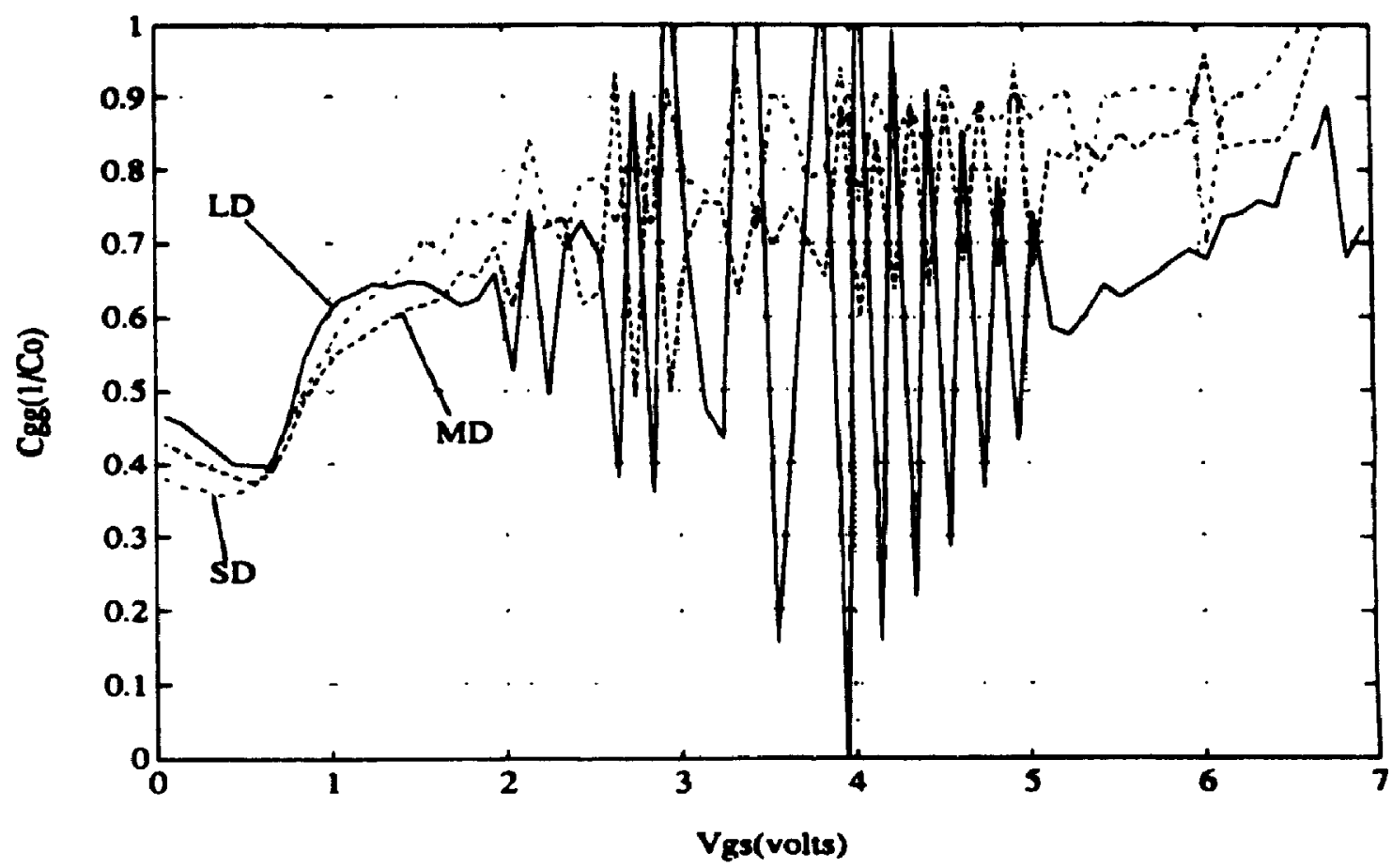

Figure 4.16 Normalized $C_{G G} ; V_{D S}=4.0 \mathrm{~V}$

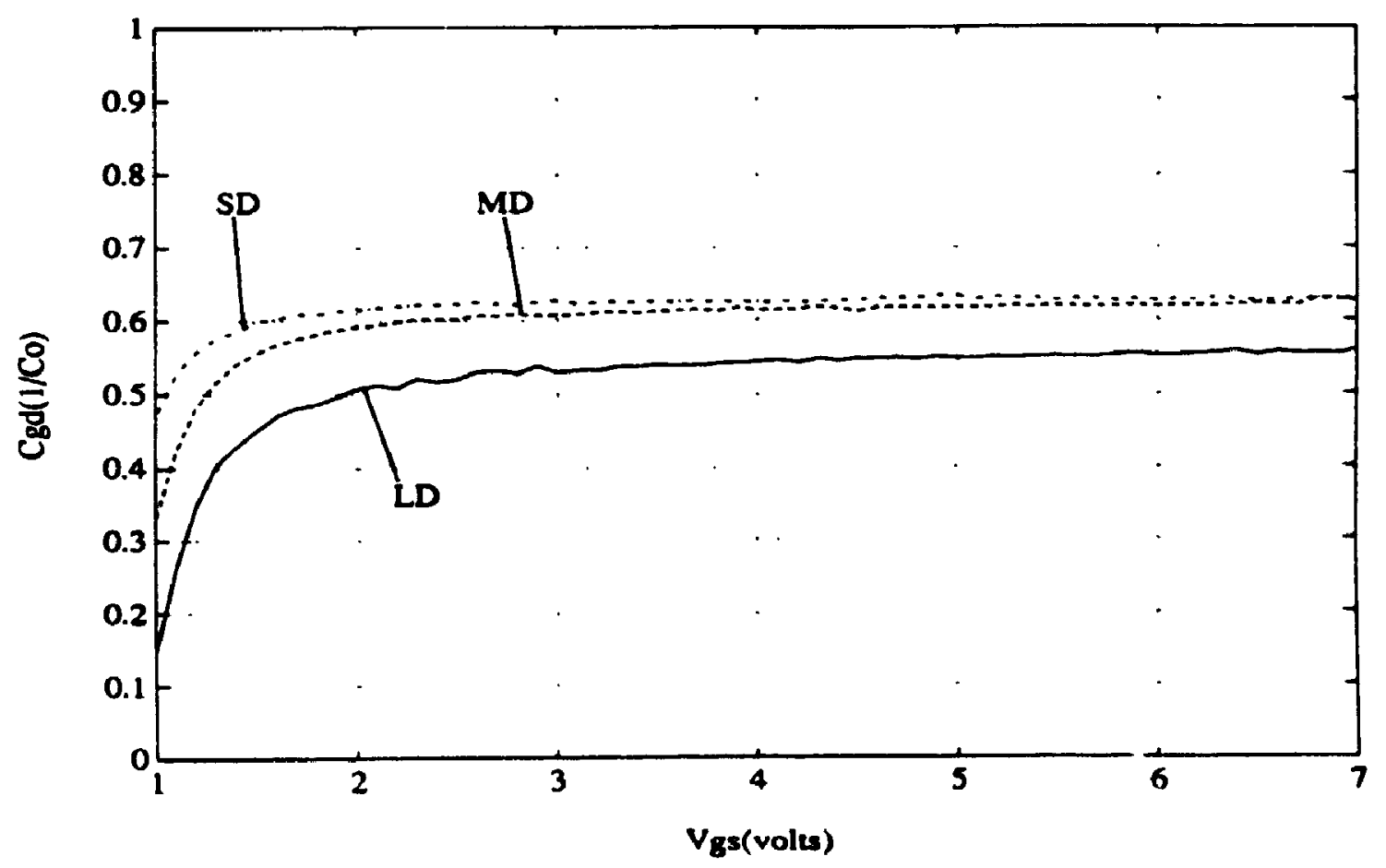

Figure 4.17 Normalized $C_{G D} ; V_{D S}=0.15 \mathrm{~V}$ 


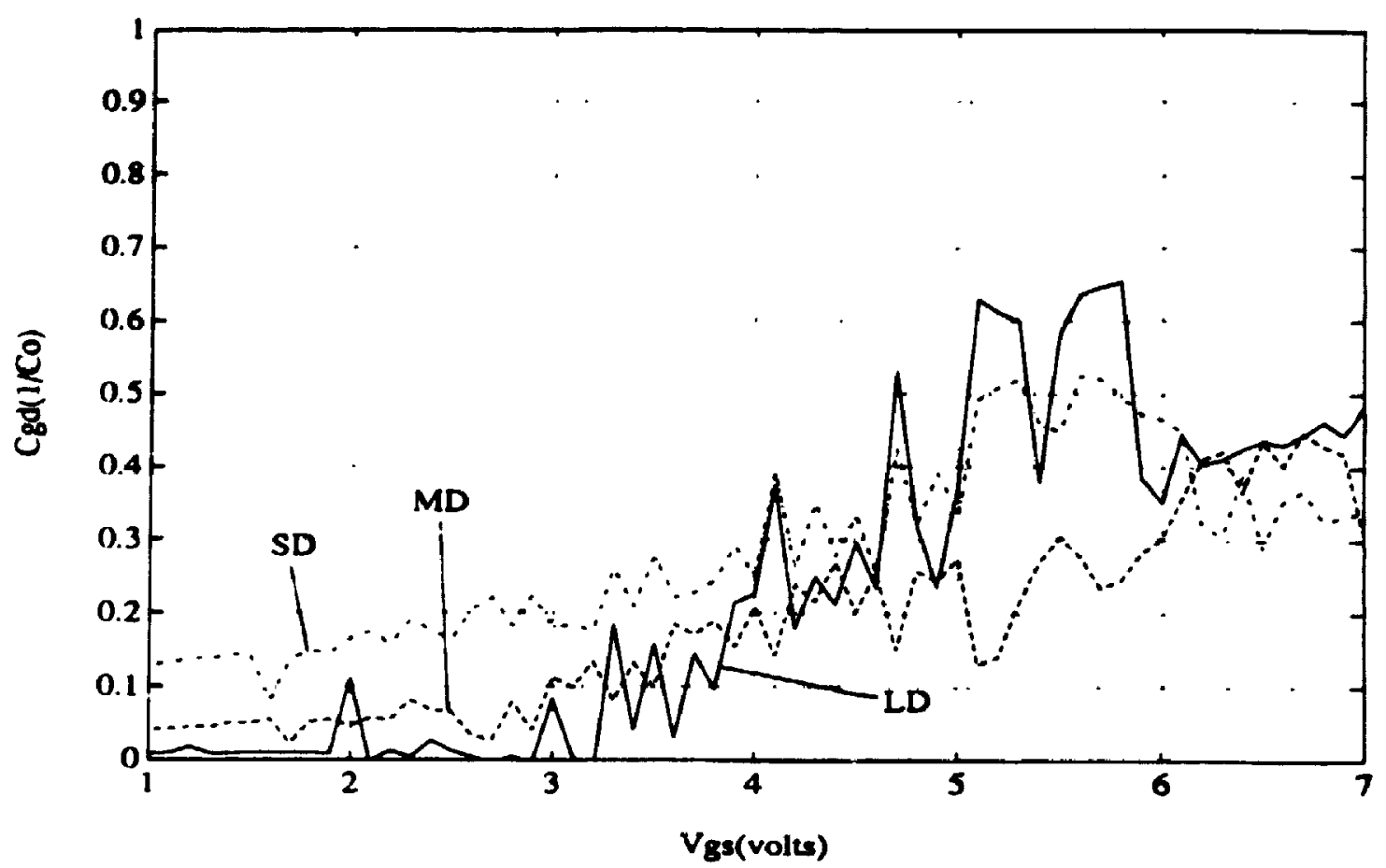

Figure 4.18 Normalized $C_{G D} ; V_{D S}=2.0 \mathrm{~V}$.

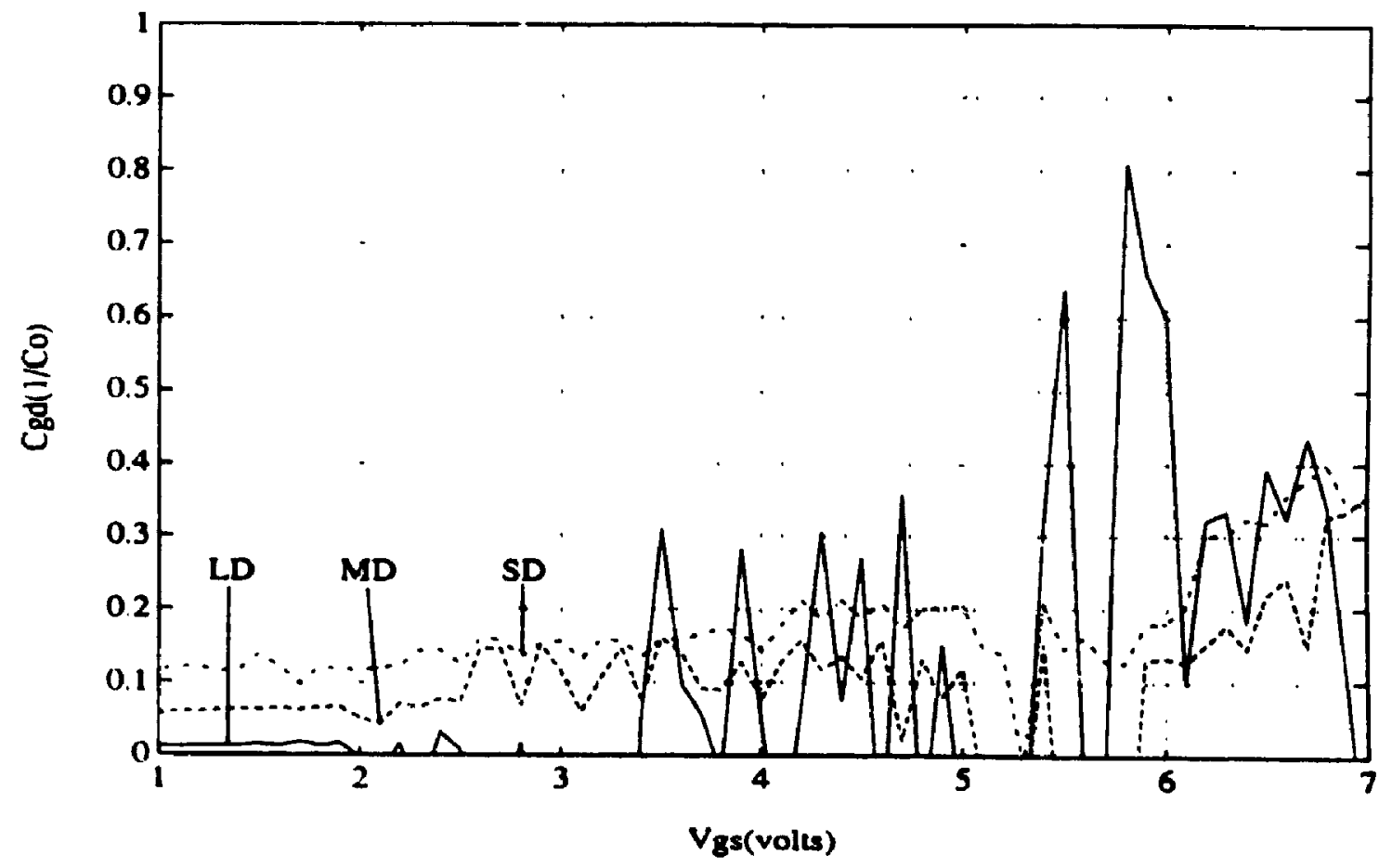

Figure 4.19 Normalized $C_{G D} ; V_{D S}=4.0 \mathrm{~V}$ 


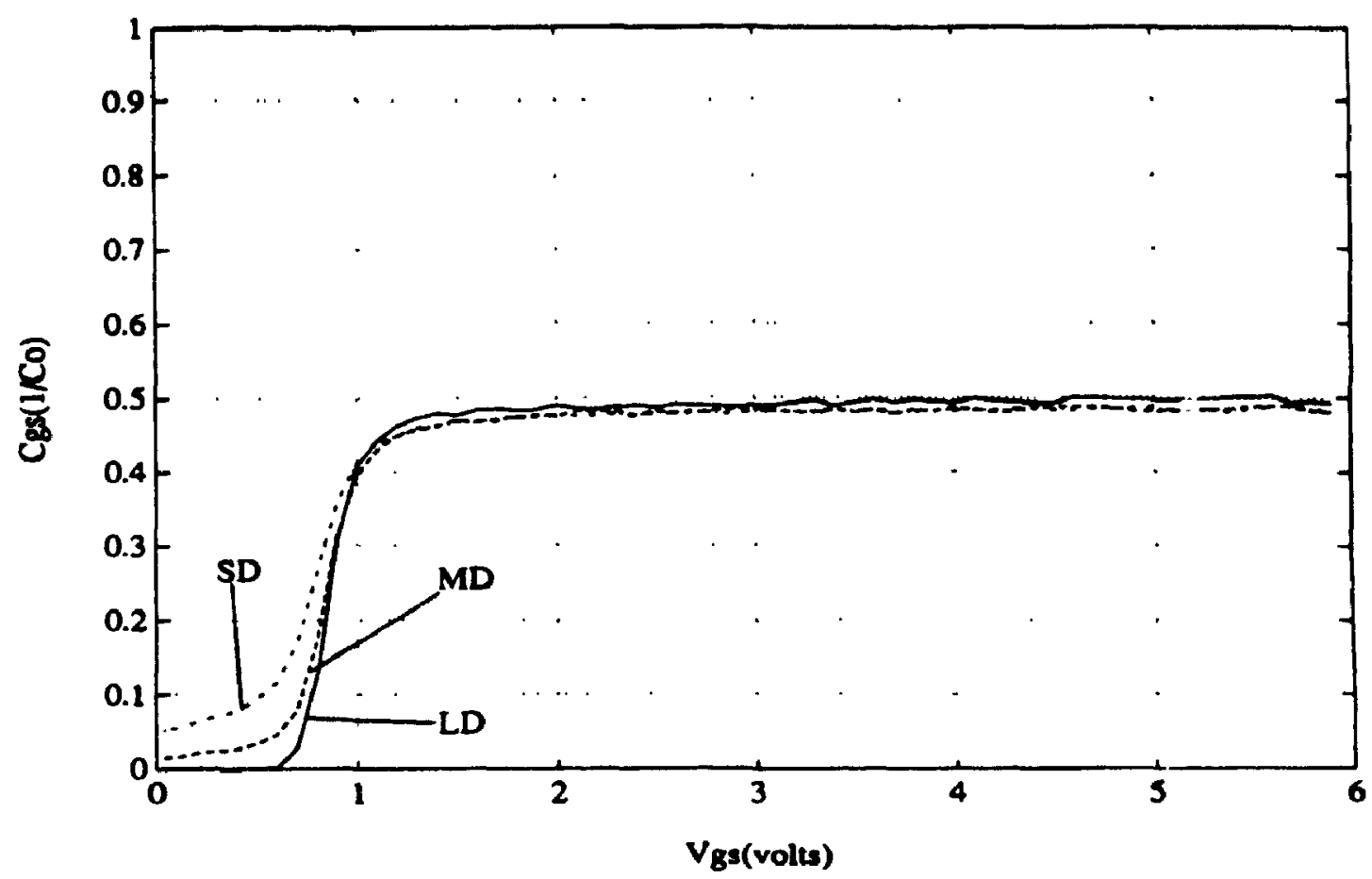

Figure 4.20 Normalized $C_{G S} ; V_{D S}=0.15 \mathrm{~V}$.

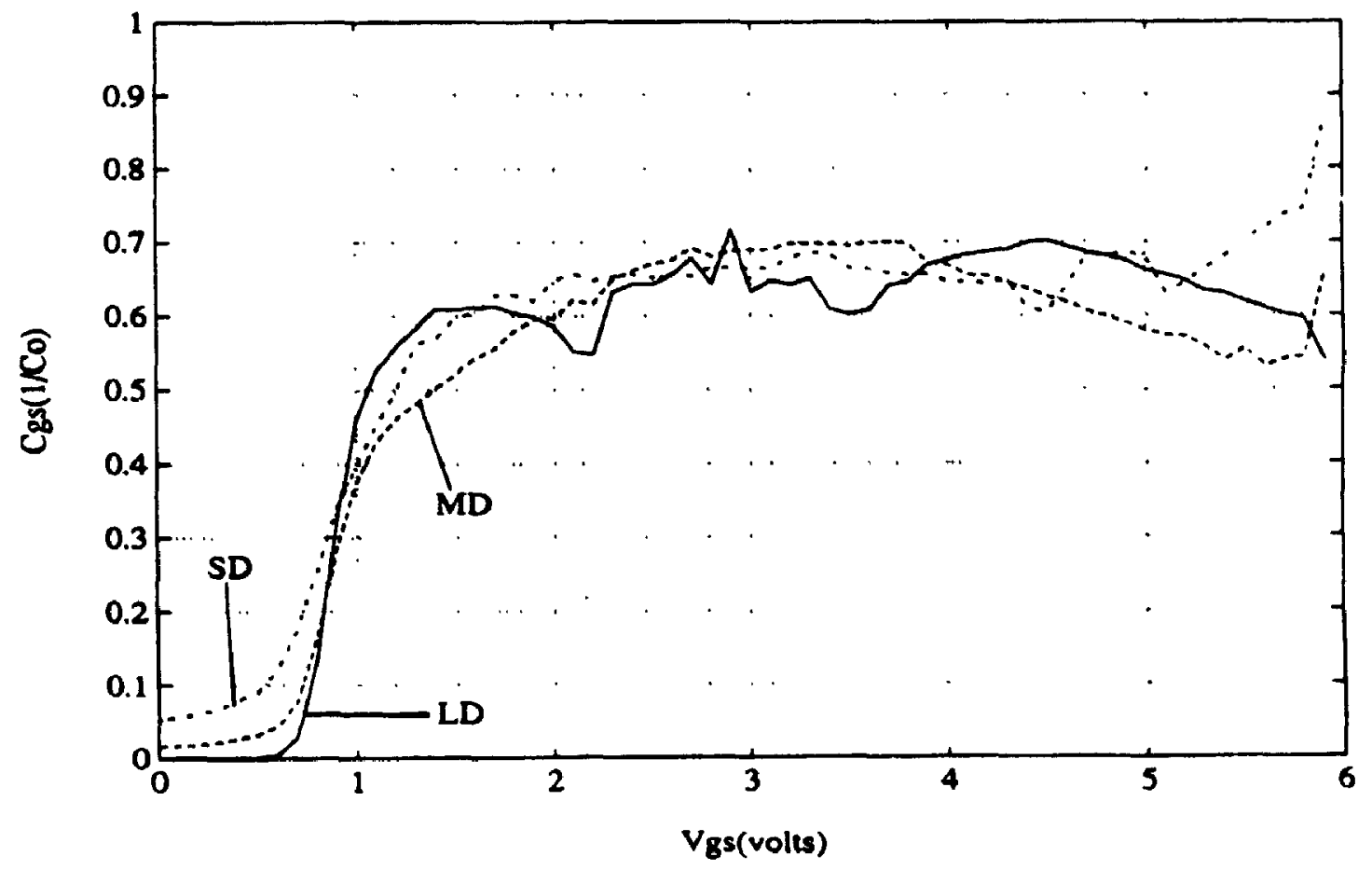

Figure 4.21 Normalized $C_{G S} ; V_{D S}=2.0 \mathrm{~V}$. 


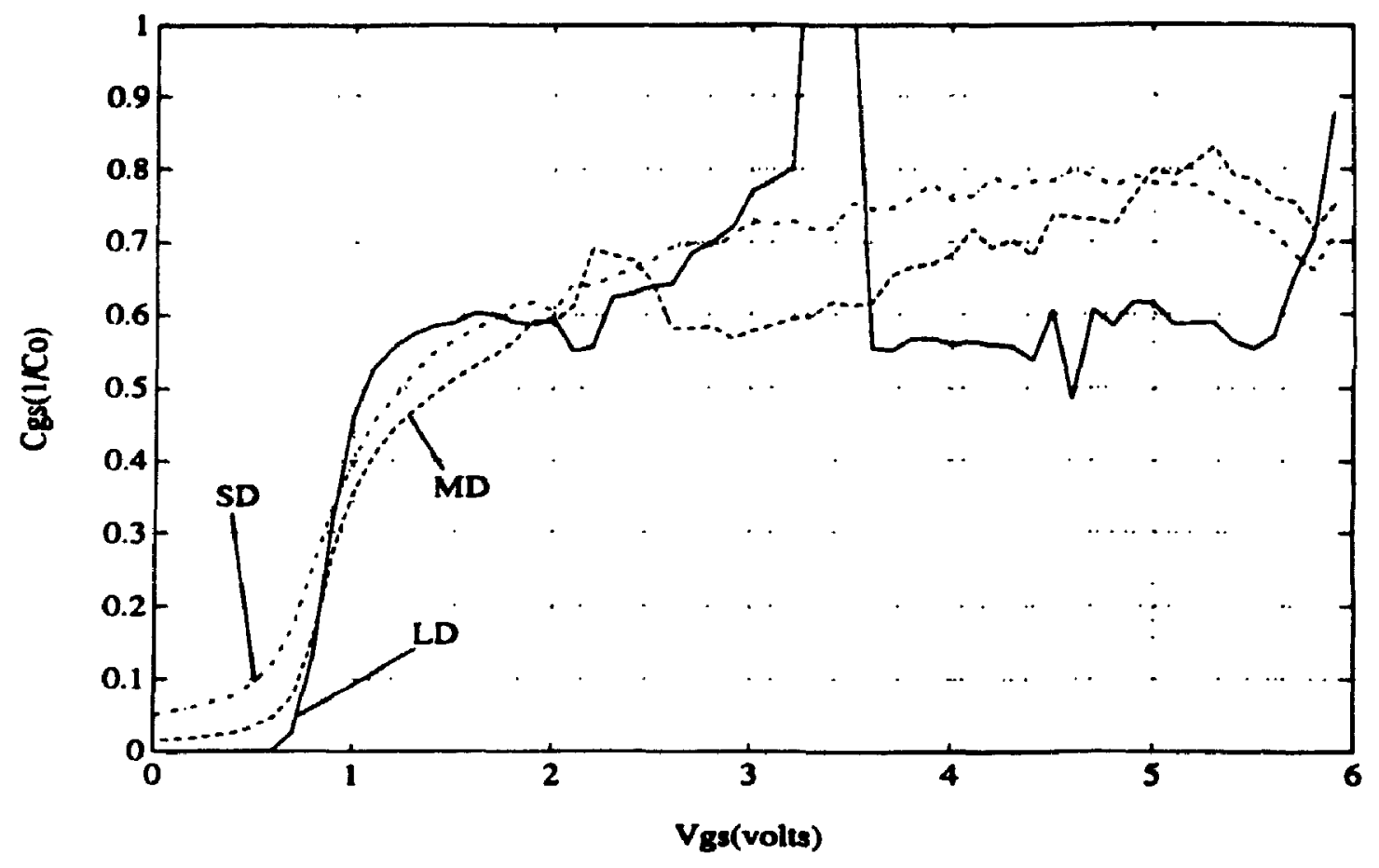

Figure 4.22 Normalized $C_{G S} ; V_{D S}=4.0 \mathrm{~V}$. 


\section{Chapter 5 \\ Analytical Modelling of Capacitance}

\subsection{Introduction}

Although MINIMOS simulation is useful for the study of charge storage and capacitance properties of short-channel MOSFETs, the vast amount of computing time involved in such 2D numerical device simulation prohibits its use in the context of device modelling for circuit analysis CAD applications. Circuit simulations require the use of simple models, preferably analytical, that are free of convergence problems and numerical scatter and whose solutions require much less computing time than involved with MINIMOS.

It is the object of the present chapter to demonstrate the application of a simple analytical model, based on the approximate representation of the surface potential distributions for the predicition of at least the first order short channel effects of short M VSFETs on the gate capacitances, and to compare the capacitance behavior given by this model with the results of MINIMOS studies in the previous chapter.

\subsection{Analytical Modelling of the 2D Surface Potential}

The analytical model which is used is based on dividing the channel into three regions and approximating the surface potential of each, as illustrated in Figure 5.1 .

The influences of the source and drain space charge regions, being two dimensional in nature, are considered separately from the center portion of the channel, which is governed by the potential at the gate alone and where the gradual channel approximation (GCA) is considered to apply. The three regions meet at the points 


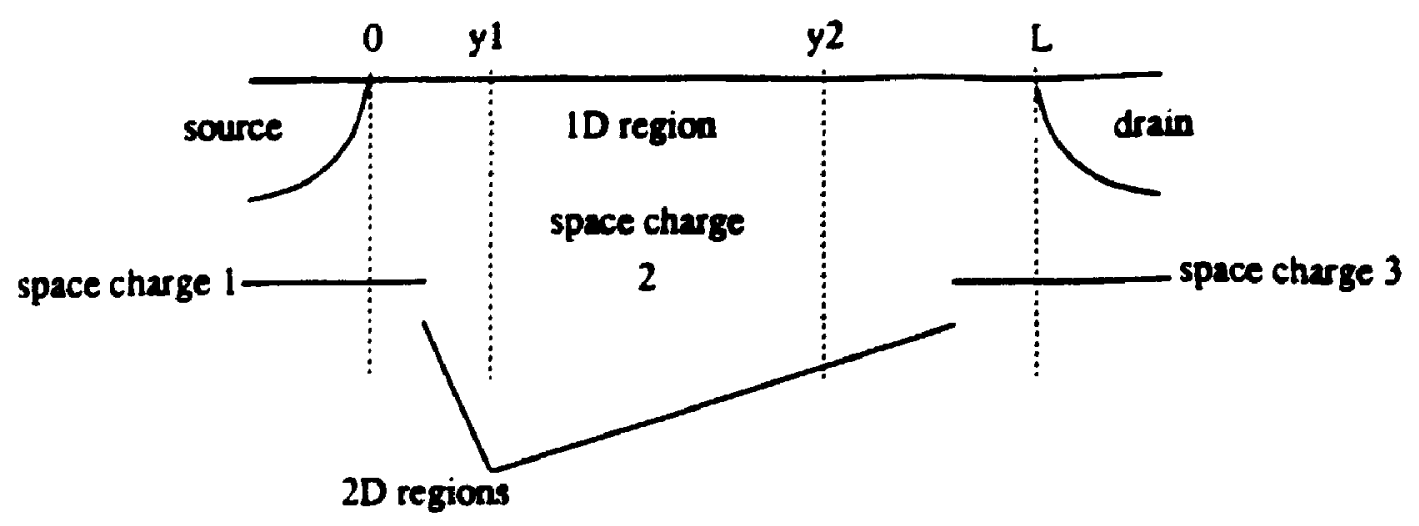

Figure 5.1 Illustration showing the division of the channel into three regions according to the analytical model (AM): the $2 D$ space charge regions of the source and drain, and the reduced $1 D$ region intersecting at points $y_{1}$ and $y_{2}$.

$y=y_{1}$ and $y=y_{2}$, which are defined as where the source and drain space charge region potentials are

$$
\begin{aligned}
& \psi_{s}\left(y_{1}\right)=\psi_{s s} \\
& \psi_{s}\left(y_{2}\right)=\psi_{s d}
\end{aligned}
$$

where $\psi_{s s}$ and $\psi_{s d}$ are the boundary potentials evaluated from long channel theory.

\subsubsection{Approximating the $\psi_{3}$-Distributions in the Source and Drain Space Charge Regions : The Cylindrical Step Junction Model}

The approach taken is to approximate $\psi_{s}(y)$ in the source and drain space charge regions by treating the source and drain as cylindrical step junctions, as illustrated in Figure 5.2, thus giving rise to the name Cylindrical Step Junction Model (CJM).

For such a representation, the source and drain space charge regions are defined strictly by the fields emanating from the edges of the cylindrical junctions, the 


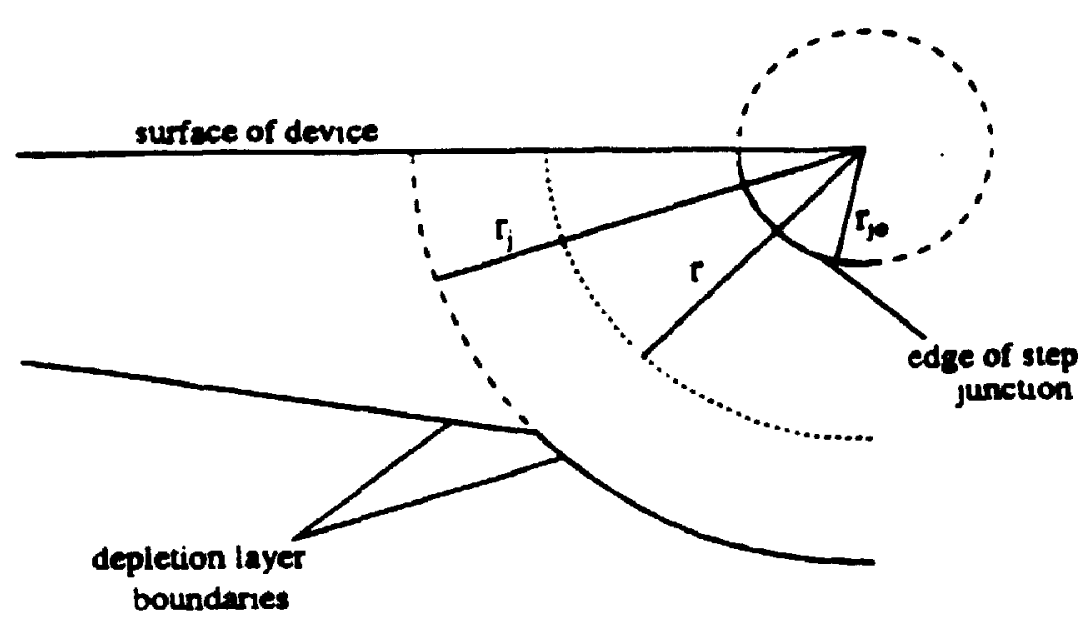

Figure 5.2 Ilustration showing the treatment of the drain (and source) as cylindrical step junctions according to the CJM.

influence of the gate being neglected. Hence, the problem has been greatly simplified from one which is two-dimensional to one whose nature is one dimensional in the radial direction.

The solution for the potential at the surface is obtained by solving Poisson's equation in cylindrical coordinates, given as

$$
\frac{1}{r} \frac{\partial}{\partial r}\left(r \frac{\partial \psi}{\partial r}\right)+\frac{1}{r^{2}} \frac{\partial^{2} \psi}{\partial \theta^{2}}+\frac{\partial^{2} \psi}{\partial z^{2}}=\frac{q N_{A}}{\epsilon_{s}}
$$

which is reduced to the 1D radial Poisson's equation due to the symmetry of the problem; that is

$$
\frac{1}{r} \frac{d}{d r}\left(r \frac{d \psi}{d r}\right)=\frac{q N_{A}}{\epsilon_{s}}
$$

and satisfies the boundary conditions:

$$
\psi(r,)=0
$$




$$
\psi\left(r_{j 0}\right)=V+\psi_{b o}
$$

where $V$ is the potential applied to the source $\left(V_{S B}\right)$ or drain $\left(V_{D B}\right), \psi_{b 0}$ is the built-in potential of the junction, $r_{j o}$ is the radius of the step junction and $r_{j}$ is defined as the edge of the depletion region, such that

$$
\left.\frac{\partial \psi}{\partial r}\right|_{r=r_{j}}=0
$$

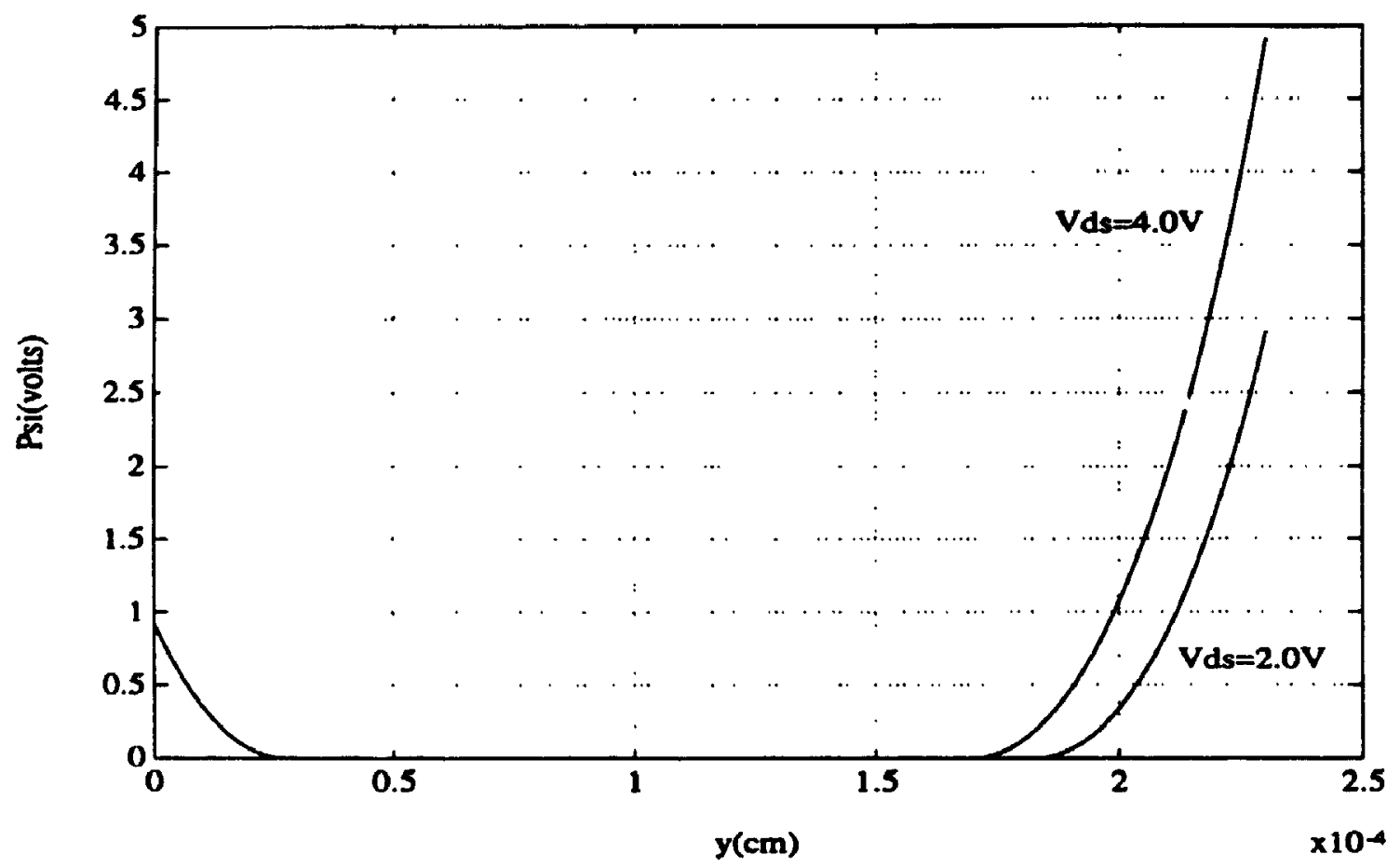

Figure 5.3 $\psi_{\mathrm{g}}$-distributions of the source and drain space charge regions, obtained from the CJM for two drain voltages, ignoring the influence of the gate.

Therefore, solving (5.2) by integration, the surface potential distribution is given by

$$
\psi(r)=\frac{q N_{A}}{2 \epsilon_{s}} r_{j}^{2}\left[\ln \left(\frac{r_{j}}{r}\right)-\frac{1}{2}\left(1-\frac{r^{2}}{r_{j}^{2}}\right)\right]
$$


Figure 5.3 shows the surface potential distributions $\psi_{0}(y)$ of the source and drain space charge regions of a device, with $N_{A}=1.4 \times 10^{16} \mathrm{~cm}^{-3}$ (as used in the MINIMOS simulations), whose effective channel length $L_{\text {eff }}=\mathbf{2 . 3} \mu \mathrm{m}$, calculated using the CJM, with $V_{S B}=0 \mathrm{~V}$ and $V_{D S}=2.0 \mathrm{~V}$ and $4.0 \mathrm{~V}$. The $\psi_{8}$-distribution at the source is strictly defined by the built-in potential $\psi_{b_{0}}$ at the junction (since the model assumes a step junction). The edge of the depletion region at the drain is shown to extend farther from the drain junction for the case of $V_{D S}=4.0 \mathrm{~V}$ as expected, due to the larger initial conditions at the junction boundary.

\subsubsection{Quadratic Approximation of $\psi_{\mathrm{s}}$-Distribution for the Reduced 1D Channel Region}

The space charge in the center portion of the channel, defined by the region $y_{1} \leq y \leq y_{2}$, is assumed to experience no influence from the source and drain, therefore being $1 \mathrm{D}$ in nature and permitting the use of long channel theory to solve for $\psi_{s}(y)$.

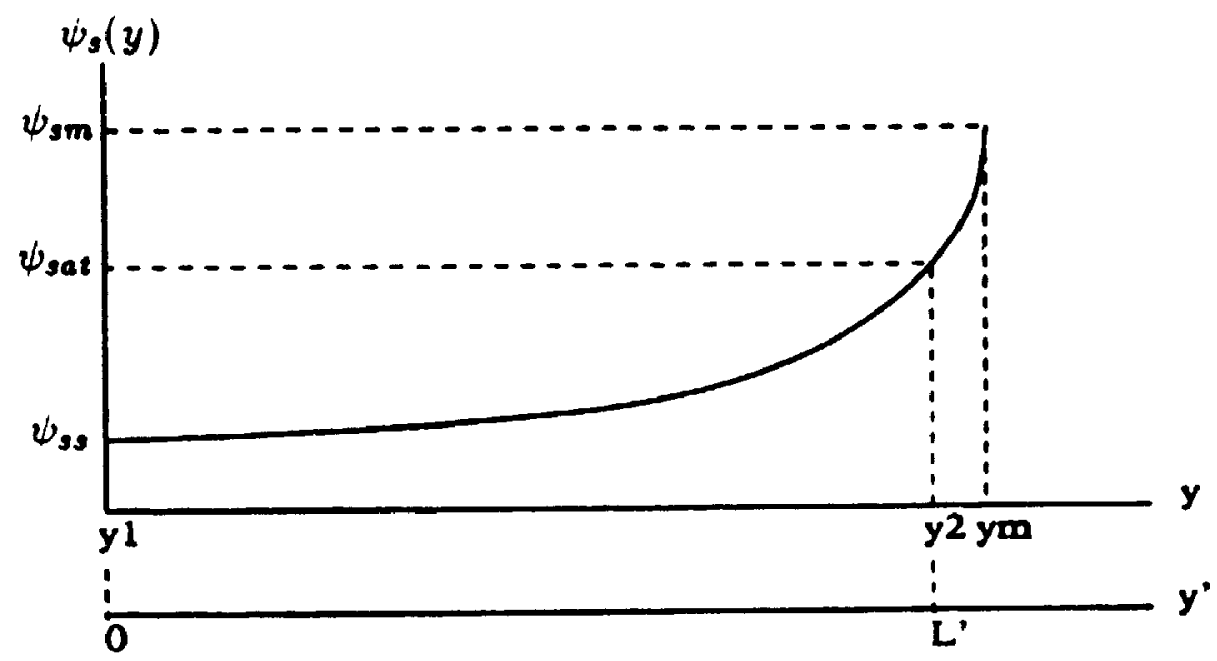

Figure 5.4 Parabola used to estimate $\psi_{2}$ in the reduced ID channel region, $y_{1} \leq y \leq y_{2}$.

Due to the complexity of the expressions from LCT, however, it is advantageous to use an approximate representation of $\psi_{\mathrm{s}}(y)$. A good approximation to the $\psi_{\mathrm{g}}$. 
distribution is a parabola such that $d \psi_{\lrcorner} / d y \rightarrow \infty$ as $\psi_{\text {sd }} \rightarrow \psi_{\text {om }}$ where, $\psi_{\text {om }}$ is defined as the maximum surface potential, corresponding to the absence of mobile charge at the surface; i.e. $Q_{M}=0[1]$, given by ${ }^{1}$

$$
\psi_{s m}=U_{g}+\frac{1}{2 a^{2}}-\sqrt{\left(U_{g}+\frac{1}{2 a^{2}}\right)^{2}-U_{g}^{2}}
$$

where

$$
\begin{gathered}
U_{g}=\left(V_{G B}-V_{F B}\right) \cdot \frac{q}{k T} \\
a=\sqrt{\frac{k T}{2 q^{2} N_{A} \epsilon_{s}}} \cdot \frac{\epsilon_{o x}}{T_{o x}}
\end{gathered}
$$

The parabola shown in Figure 5.4 may be defined as

$$
\psi_{s}\left(y^{\prime}\right)=\psi_{s m}-\left(\frac{y_{m}-y^{\prime}}{K_{y}}\right)^{\frac{1}{2}}
$$

where,

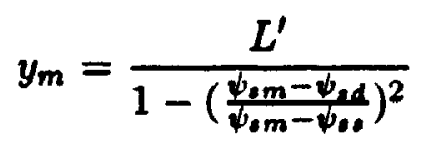

and

$$
K_{y}=\frac{L^{\prime}}{\left(\psi_{s m}-\psi_{s s}\right)^{2}-\left(\psi_{s m}-\psi_{s d}\right)^{2}}
$$

satisfying the boundary conditions $\psi_{s}(0)=\psi_{s s}$ and $\psi_{s}\left(L^{\prime}\right)=\psi_{s d}$. The origin of the primed coordinate system $\left(y^{\prime}\right)$ begins at $y=y_{1}$ and ends at $y=L^{\prime}$, which corresponds to the point $y=y_{2}$ (defining the $1 \mathrm{D}$ reduced channel region), where the surface potential $\psi^{\prime}$ sd is matched with that from the CJM distribution of the drain space change region.

A comparison of $\psi_{s}$-distributions between the quadratic approximation (QA) of (5.6) and LCT is shown in Figure 5.5 for a device with $T_{o x}=500 \AA, N_{A}=$ $1.4 \times 10^{16} \mathrm{~cm}^{-3}$ as previously, with $V_{D S}=2.0 \mathrm{~V}$ and $V_{G S}=1.5 \mathrm{~V}, 2.5 \mathrm{~V}$ and $4.0 \mathrm{~V}$.

\footnotetext{
I Equation (5.4) is obtained by making the left hand side of (5.10) to zero.
} 


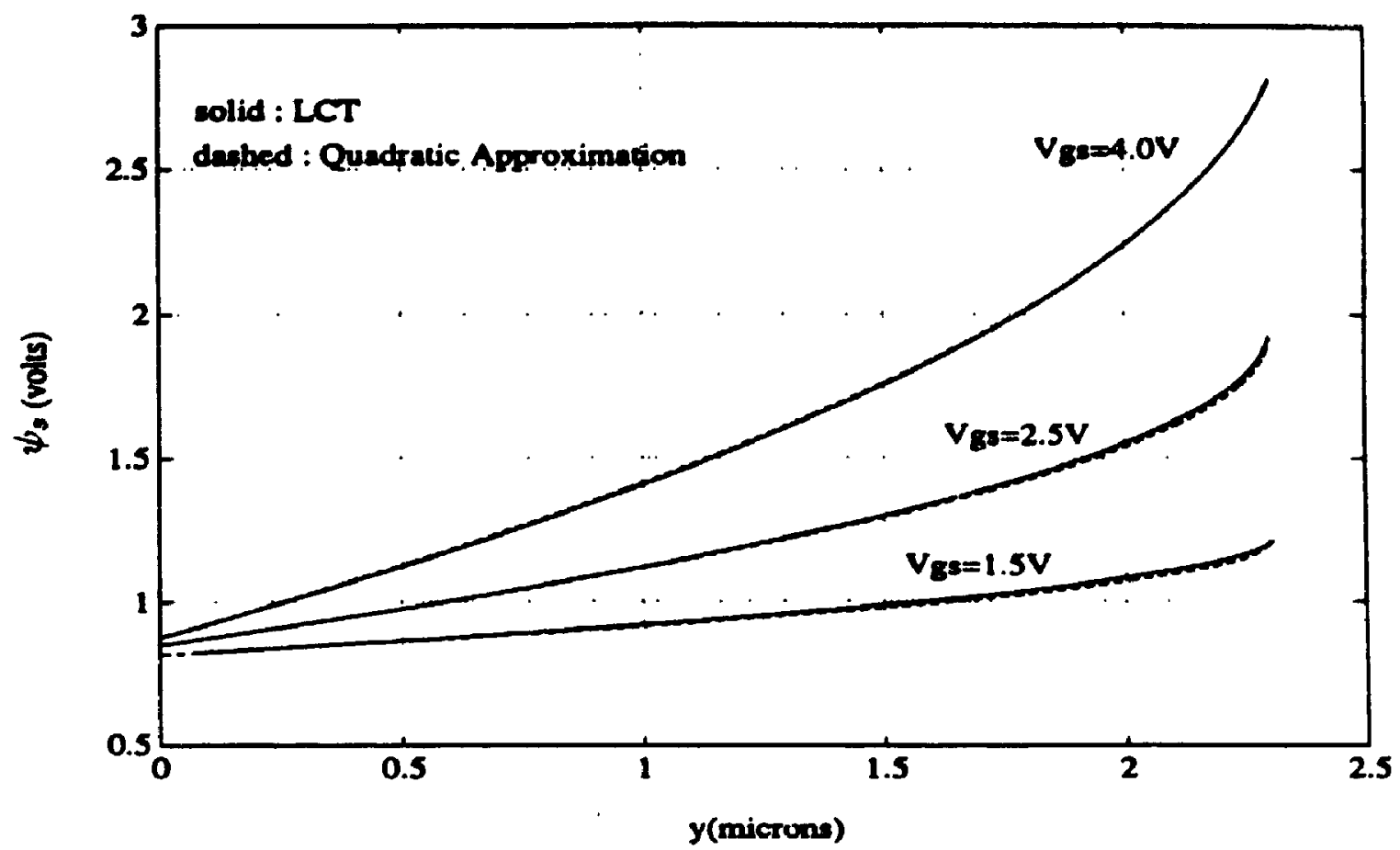

Figure 5.5 $\psi_{0}$-distributions from LCT and $Q A ; V_{D S}=2.0 \mathrm{~V}$.

For the present purpose, the length $L^{\prime}$ was taken as $2.3 \mu \mathrm{m}$. It can be seen that the QA distributions are very close to those from LCT, over most of the channel length, departing only slightly near the drain for cases operating in saturation (see the curves at $V_{G S}=1.5 \mathrm{~V}$ and $V_{G S}=2.5 \mathrm{~V}$ ). The QA distribution then falls below the LCT distributiors, thereby tending to somewhat overestimate the portion of gate charge that would be associated with the reduced channel region. When the device is operating in triode $\left(V_{G S}=4.0 \mathrm{~V}\right)$, the reverse appears to be true, with the QA distribution lying slightly above that from LCT everywhere in the channel except near the drain, thus tending to underestimate the gate charge a little.

These results are sufficient to show that the QA distribution is a feasible alternative to LCT for the 1D reduced channel region. The small differences between the two types of distribution are further reduced when the QA is used as part of the complete model, since the effective channel length of the $1 D$ center region would shrink considerably by adding the source and drain distributions; thus any 
differences in $Q_{G}$ would certainly become negligible.

\subsubsection{Division of the Channel and the Determination of the Boundaries}

As shown by Figure 5.1, the channel of the short channel MOSFET may be divided into three space charge regions whose boundaries $y_{1}$ and $y_{2}$ are defined to be points along the channel where the potential from each of the two adjacent regions is equal. Thus, the location of $y_{1}$ and $y_{2}$ should be obtained by matching the potential solutions for space charge regions 1 and 2 and regions 2 and 3, respectively.

This method however, leads to non-explicit and complex solutions of the boundaries defined by $y_{1}$ and $y_{2}$, which, for the intents and purposes of this chapter, may be estimated simply by making two further assumptions: 1) The potential at $y_{2}$ is defined from considerations of validity of the gradual channel approximation at this point; 2) the potential at $y_{1}$ is the surface potential at the source boundary in long channel theory.

Under saturation conditions in long channel theory, $\psi_{\text {sd }}$ attains the maximum value of $\psi_{s m}$, describing the classical pinch-off conditions and corresponding to $Q_{M}=0$, at the reduced channel boundary. However, for the short channel device, rather than allowing $\psi_{s d} \rightarrow \psi_{s m}$, the value of $\psi_{s d}$ is limited to a value less than $\psi_{s m}$, such that the gradual channel approximation is satisfied at $y_{2}$. Thus, for saturation conditions, $\psi_{\text {'sd }}$ is limited to a semi-empirically derived value $\psi_{\text {sat }}[2]$, defined by

$$
\psi_{s a t}-\psi_{s s}=\frac{\psi_{s m}-\psi_{s s}}{1+\frac{F T_{o x}}{L}}
$$

where $T_{o x}=500 \AA, L$ is reduced channel length $y_{2}-y_{1}$ and $F$ is a constant, chosen to be $\gg 1$; for the physical parameters of the device used, $F=30$ is sujtable[2]. Figure 5.6 shows $\psi_{s s}, \psi_{s m}$ and $\psi_{s a t}$ over the range of gate voltages where the device is operating in the subthreshold and saturation regions for $V_{D S}=1.95 \mathrm{~V}$. It should be noted that as subthreshold conditions are approached (recalling $V_{T H}=0.745 \mathrm{~V}$, as specified in Chapter 3) $\psi_{s s} \rightarrow \psi_{s m}$ and also $\psi_{s a t} \rightarrow \psi_{s m}$. 


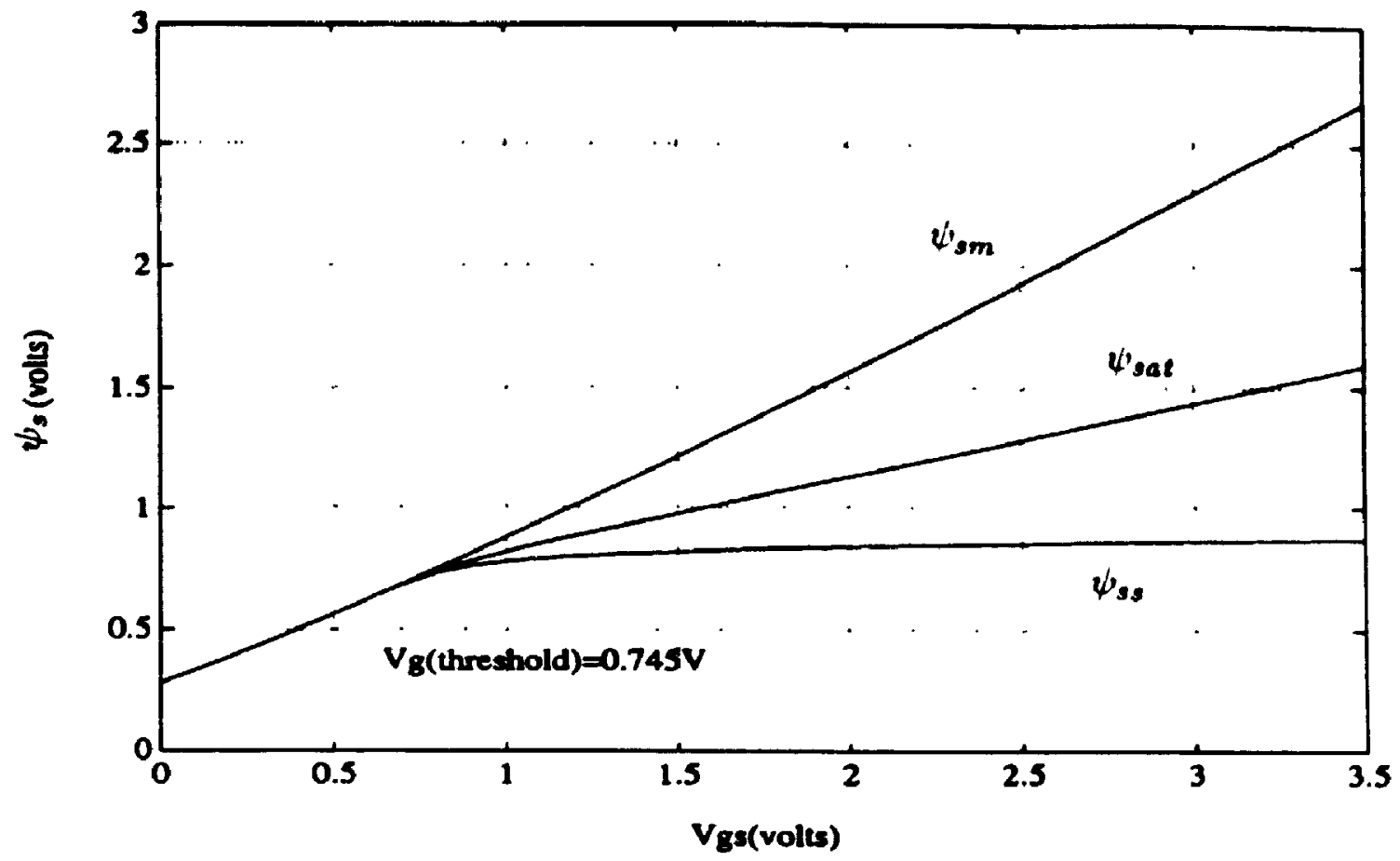

Figure 5.6 Distributions of $\psi_{s m}, \psi_{s a t}, \psi_{s e} ; V_{D S}=2.0 \mathrm{~V}$

The potential $\psi_{s s}$ ( and consequently the location of $y_{1}$, found from the CJM distribution at the source) may be estimated by solving the surface equation from LCT, used to obtain the boundary potential at the edges of the channel[1]; that is

$$
e^{U_{s}-\xi-2 U_{S}}=a^{2}\left(U_{g}-U_{s}\right)^{2}-U_{s}
$$

$\xi=V \cdot q / k T$, (with, $V=V_{S B}$ or $V_{D B}$ ) and all other voltages are normalized by $k T / q$; here, for $N_{A}=1.4 \times 10^{16} \mathrm{~cm}^{-3}, T_{o x}=500 \AA, a=0.1617$. Equation (5.10) is readily solved numerically.

The error introduced by estimating $\psi_{s s}$ and the location $y_{1}$ using this method, with respect to the actual values evaluated from the solution of the potential distribution of the space charge regions 1 and 2 is expected to be negligible, since the distribution of space charge region 1 makes a minor contribution to the total distribution $\psi_{s}$, the integral of which is related to the total gate charge - as can be observed from Figure 5.7, which shows two different distributions for a device 


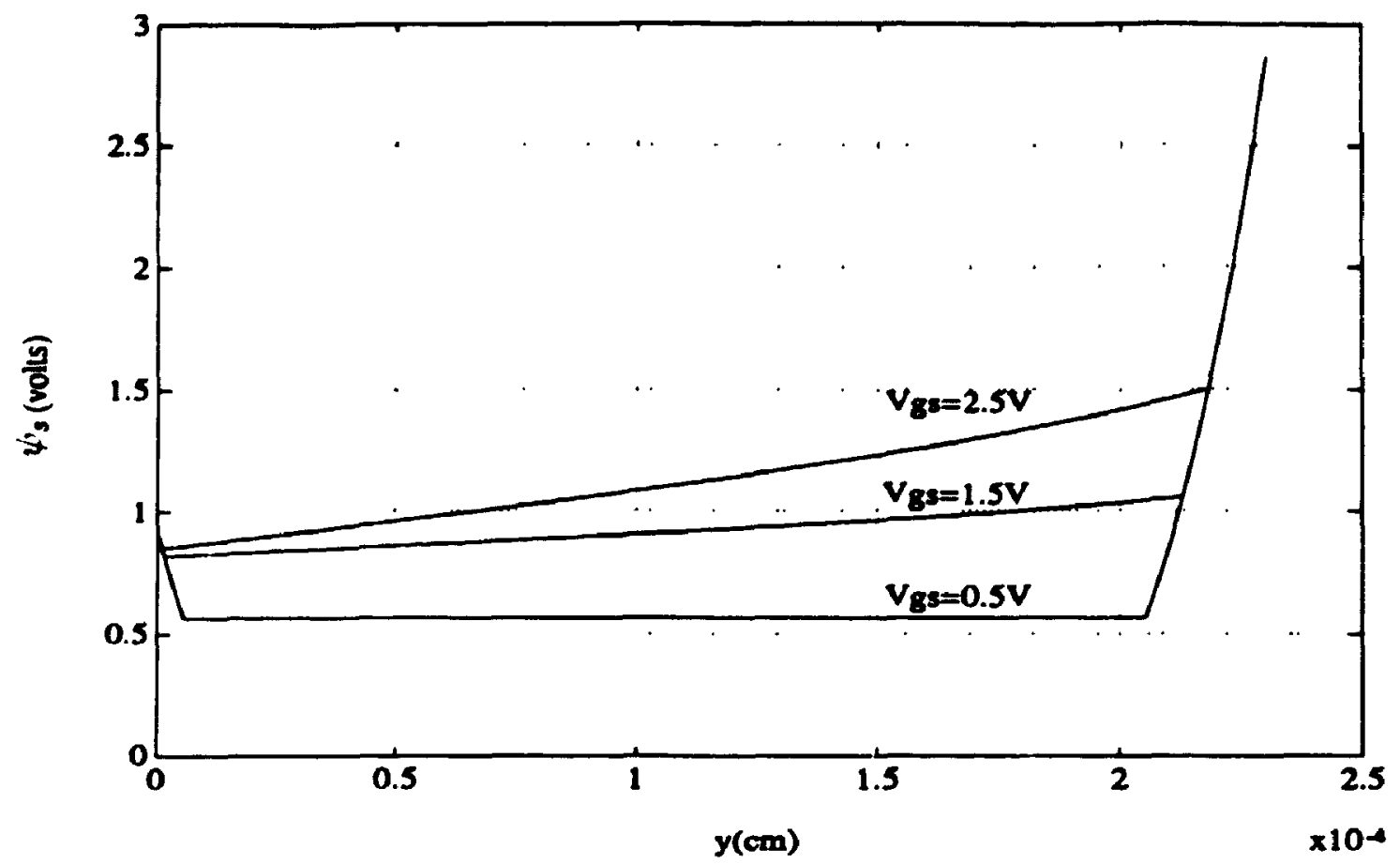

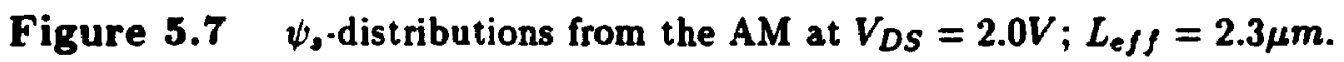

operating in saturation with $V_{G S}=1.5 \mathrm{~V}$ and $V_{G S}=2.5 \mathrm{~V}$ at $V_{D S}=1.95 \mathrm{~V}$. The figure clearly demonstrates the decrease in the influence of the source and drain space charge regions at the device is made to operate towards the triode region: by increasing $V_{G S}$, and thereby increasing the length $L^{\prime}$ of the reduced 1D channel region by moving the points $y_{1}$ and $y_{2}$ further apart, the contribution of $\psi_{s}(y)$ from the CJM distributions of the source and drain is reduced.

\subsection{Gate Capacitance Component $C_{G D}$}

\subsubsection{Evaluation of $C_{G D}$ Using Analytical Approach}

As shown in Figure 5.7, the surface potential distribution of the channel is divided into three regions, defined by the boundaries $\psi_{s a}$ and $\psi_{\text {sat }}$ at $y=y_{1}$ and $y=y_{2}$, for a given set of bias conditions. The gate charge $Q_{G}$ induced in the channel 
must be evaluated by adding the contributions of gate charge $Q_{G}(s), Q_{G}(c h)$ and $Q_{G}(d)$ of the source, reduced ID channel and drain space charge regions respectively. which in turn are obtained by integrating the corresponding distributions of $V_{G B^{\prime}}$ $\psi_{s}(y)$, where $V_{G B}{ }^{\prime}=V_{G B}-V_{F B}$ and $V_{F B}$ is the flatband voltage. $C_{G D}$ is therefore defined as

$$
C_{G D}=-\left.\frac{\left(Q_{G 2}-Q_{G 1}\right)}{\Delta V_{D S}}\right|_{V_{G S}}
$$

where, $Q_{G 1}$ and $Q_{G 2}$ are the total gate charges evaluated at $V_{D S 1}$ and $V_{D S 2}$ respectively, and $\Delta V_{D S}=V_{D S 2}-V_{D S 1}$, for a given $V_{G S}$. The integrals were performed numerically using Simpson's $\frac{1}{3}$ Rule with $10^{4}$ points.

The $C_{G D}$ characteristics obtained for the medium $\left(L_{e f f}=2.3 \mu m\right)$ and short $\left(L_{\text {eff }}=1.0 \mu \mathrm{m}\right)$ devices operating at $V_{D S}=2.0 \mathrm{~V}$ and $4.0 \mathrm{~V}$ are shown in Figure 5.8. The range of gate voltages was chosen to cover saturation extending down into subthreshold, because, as mentioned in the previous section, the boundary condition at $y=y_{2}$ is defined only for the subthreshold and saturation regions by $\psi_{s d}=\psi_{s a t}$, where $\psi_{s d}<\psi_{s m}$ to ensure that the GCA applies everywhere inside the reduced $1 \mathrm{D}$ channel.

The most striking feature of the $C_{G D}$ characteristics is that the capacitunce tends to decrease as $V_{G S}$ increases. This behavior can be understood by observing the $\psi_{9}$-distributions in Figure 5.9, shown for two gate voltages. The difference in $V_{D S}$ between the two distributions at the drain has been exaggerated in order to increase the difference in their displacement and simplify the demonstration. At low $V_{G S}$ the cylindrical distributions of $\psi_{s}(y)$ from the drain extend well into the channel, thereby contributing a significant portion of the total distribution, corresponding to a large change in gate charge, associated with the difference in area between the distributions at the two values of $V_{D S}$. As the gate voltage is increased, the contribution from the drain space charge region decreases, ther sore reducing the change in gate charge $\Delta Q_{G}$.

This behavior can also be demonstrated from Figure 5.10, which shows the 


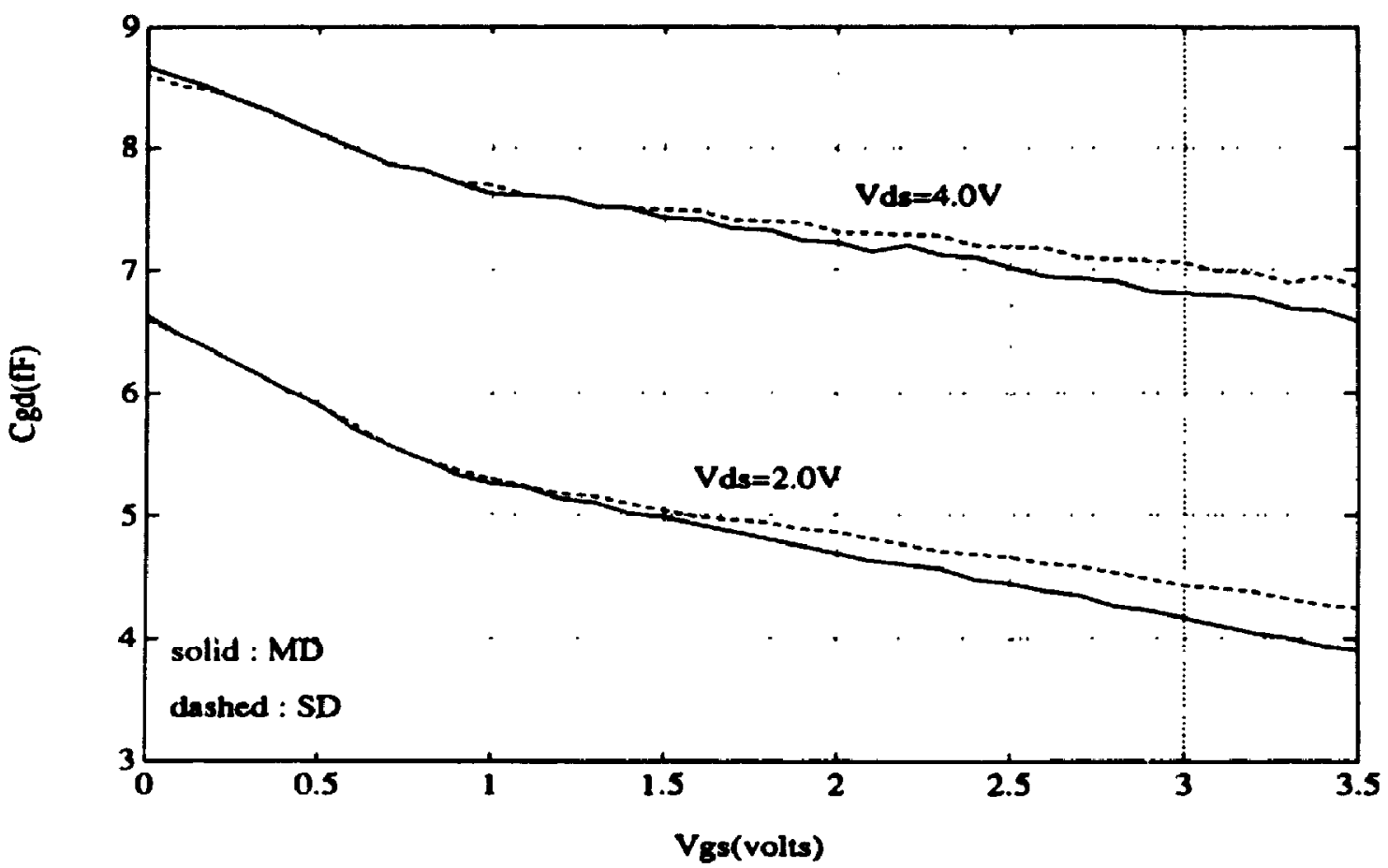

Figure 5.8 $C_{G D} \sim V_{G S}$ from the $\mathrm{AM} ; L_{\text {eff }}=2.3 \mu \mathrm{m}$ and $L_{\text {eff }}=1.0 \mu \mathrm{m}$.

distribution of the total gate charge $Q_{G}$ with $V_{G S}$ for the same devices and operating conditions as in Figure 5.8. The curves of both the MD and SD, at the two values of $V_{D S}$, are further apart at low $V_{G S}$, comming closer together as the gate voltage increases.

In both figures, it is observed that there is a change of slope in the characteristics, occuring at around threshold. Starting with low $V_{G S}$ values, the $C_{G D}$ curves of both devices appear to overlap for both values $V_{D S}$ until threshold is reached, at which point the curves of the SD level off more, while those of the larger device continue to decrease after experiencing a smaller bend. The bending of the $C_{G D}$ characteristics at around threshold occurs because of the analytical condition chosen to define 4 'sat at $y_{2}$, given by (5.9). As shown earlier in Figure 5.6, as $V_{G S}$ approaches the subthreshold region from higher values, $\psi_{\text {sat }} \rightarrow \psi_{\text {ss. }}$. Therefore, the area under the $s_{s}(y)$ distribution associated with $Q_{G}$, will begin to change at a 


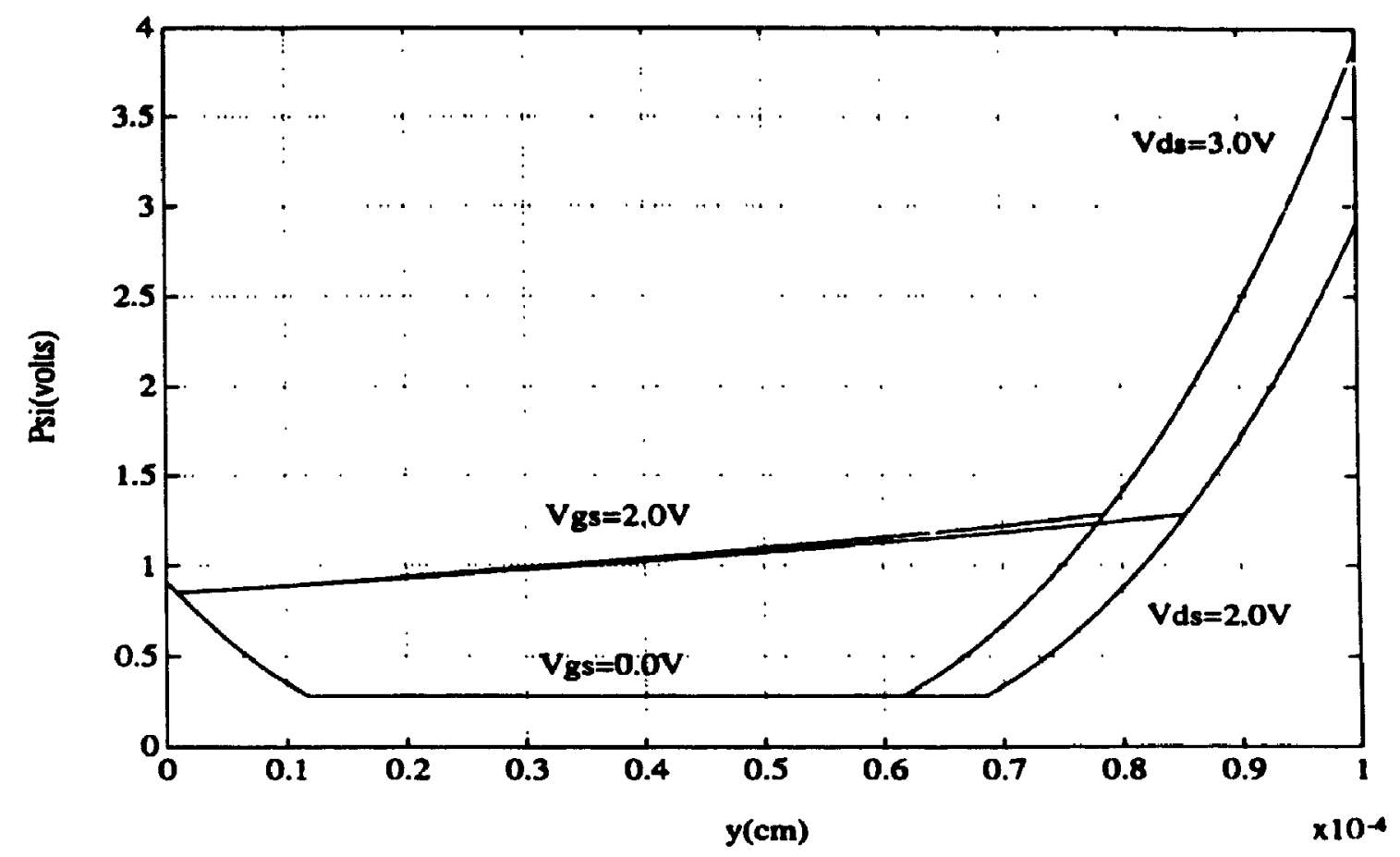

Figure 5.9 Difference between two $\psi_{\ell}$-distributions for different drain voltages; $L_{\text {eff }}=1.0 \mu \mathrm{m}$.

different rate in the subthreshold where, $\psi_{\text {sat }} \simeq \psi_{s s}$, than in the saturation region, where $\psi_{\text {sat }}>\psi_{s s}$.

The separation between the $C_{G D}$ characteristics of the MD and the SD may be explained as follows : In the case of the larger device, it is clear that there is a smaller contribution to the total gate charge $Q_{G}$ from the drain space charge region, due to the influence of channel length $L$ on the saturation condition of (5.9). As $L$ is reduced, $\psi_{s a t} \rightarrow \psi_{s s}$ and the extent $L-y_{2}$ of the drain space charge region expands. Thus, as $L$ is increased, the contribution of the drain space charge region to $\Delta Q_{G}$ is reduced and $C_{G D}$ is reduced accordingly for the same value of $V_{G S}$ above threshold. Below threshold, $\psi_{s a t}=\psi_{s s}$, and there is no contribution to the charge difference $\Delta Q_{G}$ due to length dependence of the $1 D$ region of the MD associated with the saturation condition, and therefore $C_{G D}$ is essentially the same for both devices. 


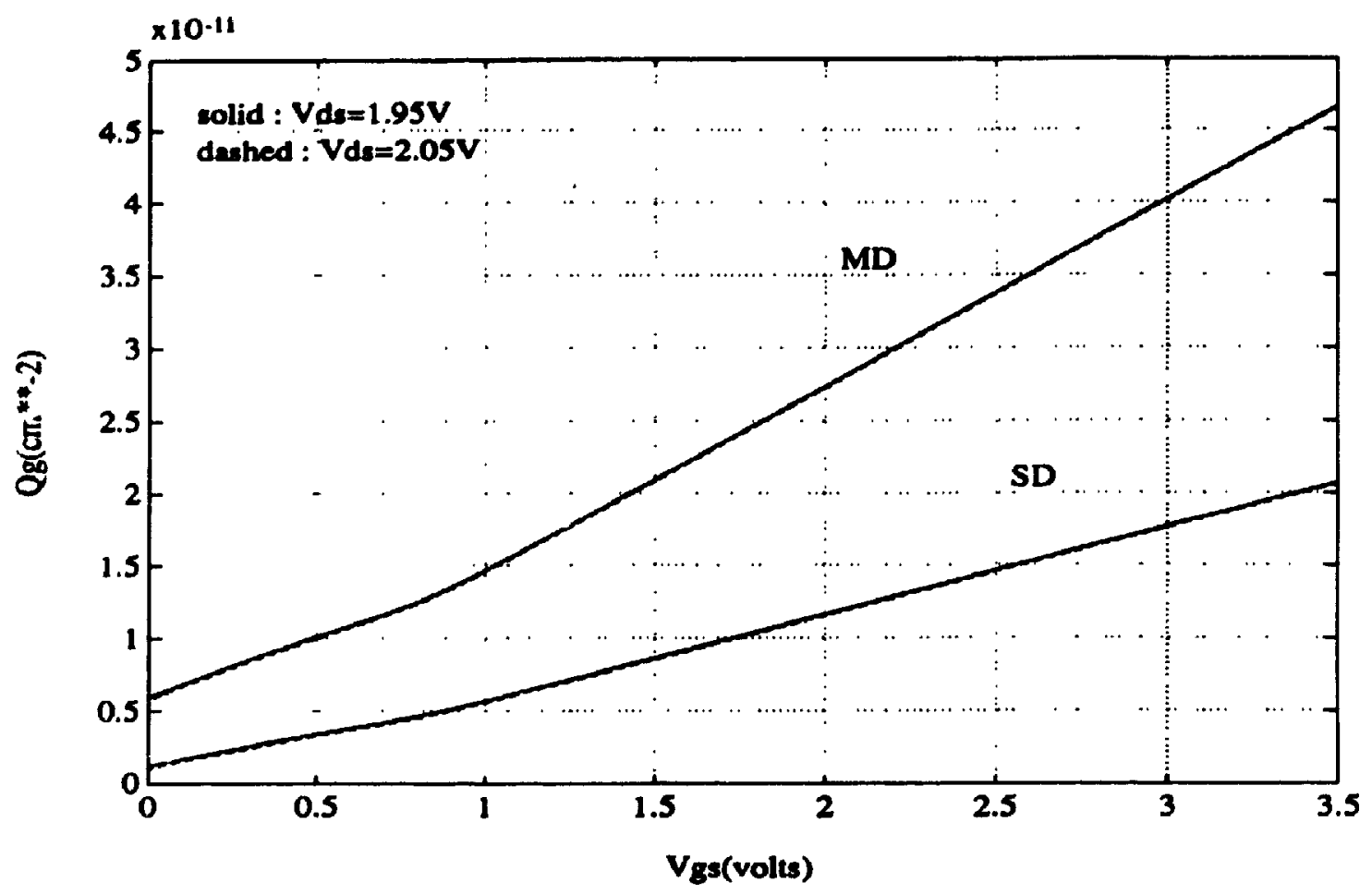

Figure 5.10 Distributions of $Q_{G}$ for the 1D channel region; $L_{\text {eff }}=2.3 \mu \mathrm{m}$ and $L_{\text {eff }}=1.0 \mu m$.

A related effect due to the separation of the boundary point $y_{2}$, between the two devices, is as follows: since $y_{2}$ is closer to $L$ for the larger device, $\psi_{\text {sat }}$ begins to rise above $\psi_{s}$ at a lower gate voltage, which should cause the curves of the MD to bend at a lower gate voltage. This, however, is not noticeable from Figure 5.8 because of the scatter introduced from the numerical intergration used to evaluate $Q_{(i}$

The difference in the contribution of the drain space charge region $Q_{G}(d)$, between the two devices is shown in Figure 5.11. It is observed that below threshold, the two pairs of curves (each corresponding to the two drain voltages at which $C_{G D}$ is evaluated) overlap, since the location of the drain boundary $y_{2}$ is identical for both devices. As $V_{G S}$ is increased above threshold, $\psi_{\text {sat }}$, and thus the correspondIng location $y_{2}$, begins to differ for the two devices, hence causing the separation between the two pair of curves. The $C_{G D}$ characteristics of the MD drop below 
those of the SD above threshold, corresponding to the smaller contribution of the drain region of the larger device.

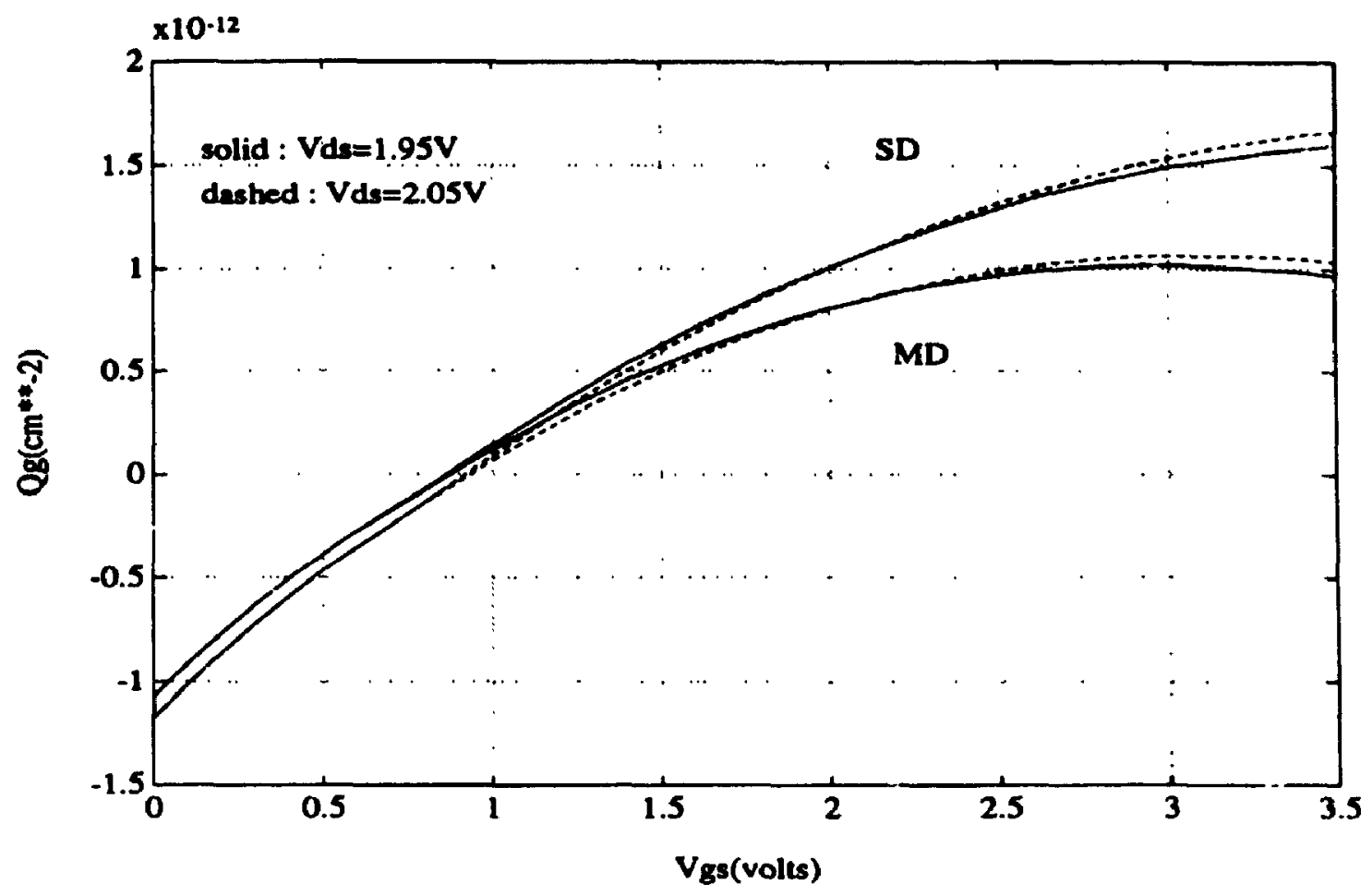

Figure 5.11 Distributions of $Q_{G}$ for the drain space charge region; $L_{e f s}=2.3 \mu \mathrm{m}$ and $L_{\text {eff }}=1.0 \mu \mathrm{m}$.

It should also be noted from Figure 5.11 that at lower gate voltages, the values of $Q_{G}(d)$ are negative. In the drain space charge region

$$
Q_{G}(d)=C_{o x} \int_{y_{2}}^{L}\left(V_{G B^{\prime}}-\psi_{s}(y)\right) d y
$$

which results in negative charge where the value of $\psi_{s}(y)$ near the drain is greater than $V_{G B}{ }^{\prime}$.

It is clear therefore, that the model predicts a non-zero capacitance for the subthreshold and saturation regions, because of the difference in the distributions $\psi_{s}(y)$ of the drain space charge region, as $V_{D S}$ is modulated. If this region were 
neglected, the situation would be reduced to the classical long channel case where the capacitance $C_{G D}$ is zero.

\subsubsection{Comparison of Analytical Results with those from MINIMOS}

Normalized $C_{G D}$ characteristics obtained by MINIMOS simulation, and by use of the analytical model (AM), are shown in Figures 5.12(a) and (b), for the MD and SD respectively, at $V_{D S}=2.0 \mathrm{~V}$ and $4.0 \mathrm{~V}$. The first and foremost feature that should be noted is that both methods predict the presence fo non-zero capacitance $C_{G D}$ in the subthreshold and saturation regions of operation, as well as values that are similar in magnitude. As discussed in Sections 4.3 and 4.4 of the previous chapter, MINIMOS, with its 2D representation of the surface potential, is capable of revealing the presence of the small inner fringing capacitance component $C_{G D(i f)}$ between the gate and drain, whose significance was shown to increase as the dimensions of the device were reduced. This weak coupling capacitance is also identified by the analytical model, which, despite its simplicity, is capable of predicting the significant differences in the distribution of $\psi_{s}(y)$ as the drain bias is modulated. However, in the analytical model the influence of the gate voltage in the surface potential in the drain space charge region, and its corresponding effect in $C_{G D(i f)}$, is not represented, as discussed further below.

Figure 5.13 compares the surface potential distributions $\psi_{s}(y)$ obtained from MINIMOS with those from the analytical model, for the MD with $V_{D S}=4.0$ ?, operating in the subthreshold $\left(V_{G S}=0.35 \mathrm{~V}\right)$ and saturation regions $\left(V_{G S}=2.05 \mathrm{~V}\right.$ and $3.05 \mathrm{~V}$ ). One of the assumptions made by the analytical model of the MOSFET is that the source and drain are step junctic .s; consequently, the $\psi_{\mathbf{s}}$-distribution appears markedly different from that of MINIMOS, which is able to show the gradual change in $\psi^{\prime} s(y)$ at the source and drain corresponding to the gradual transition of the doping profile in these regions. The potential $V+\psi_{b o}$, at the edges of the channel (where, $V=V_{S B}$ at the source end and $V_{D B}$ at the drain end), actually 


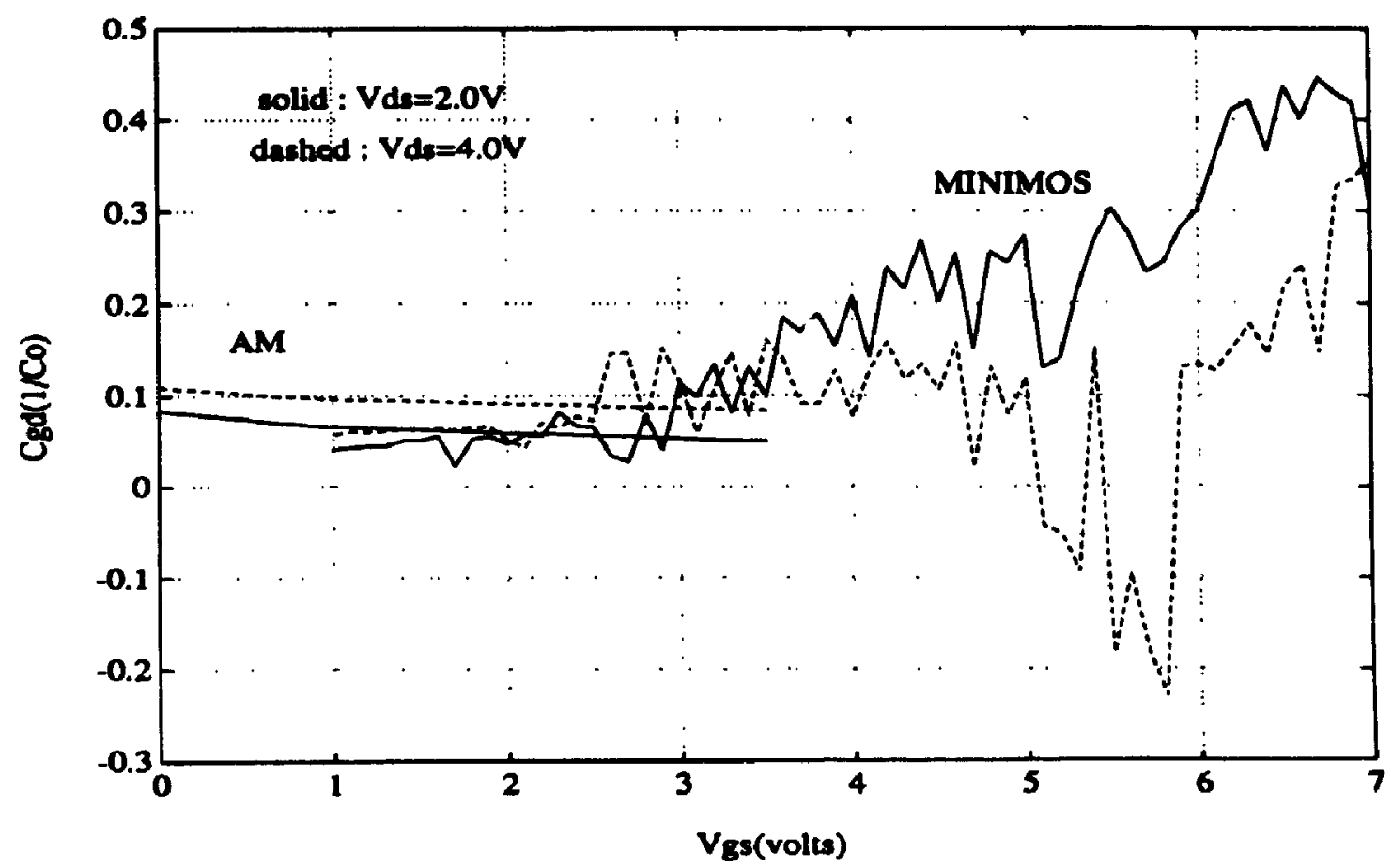

Figure 5.12(a) Normalized $C_{G D}$ obtained from MINIMOS and the AM; $L_{e f f}=2.3 \mu m$.

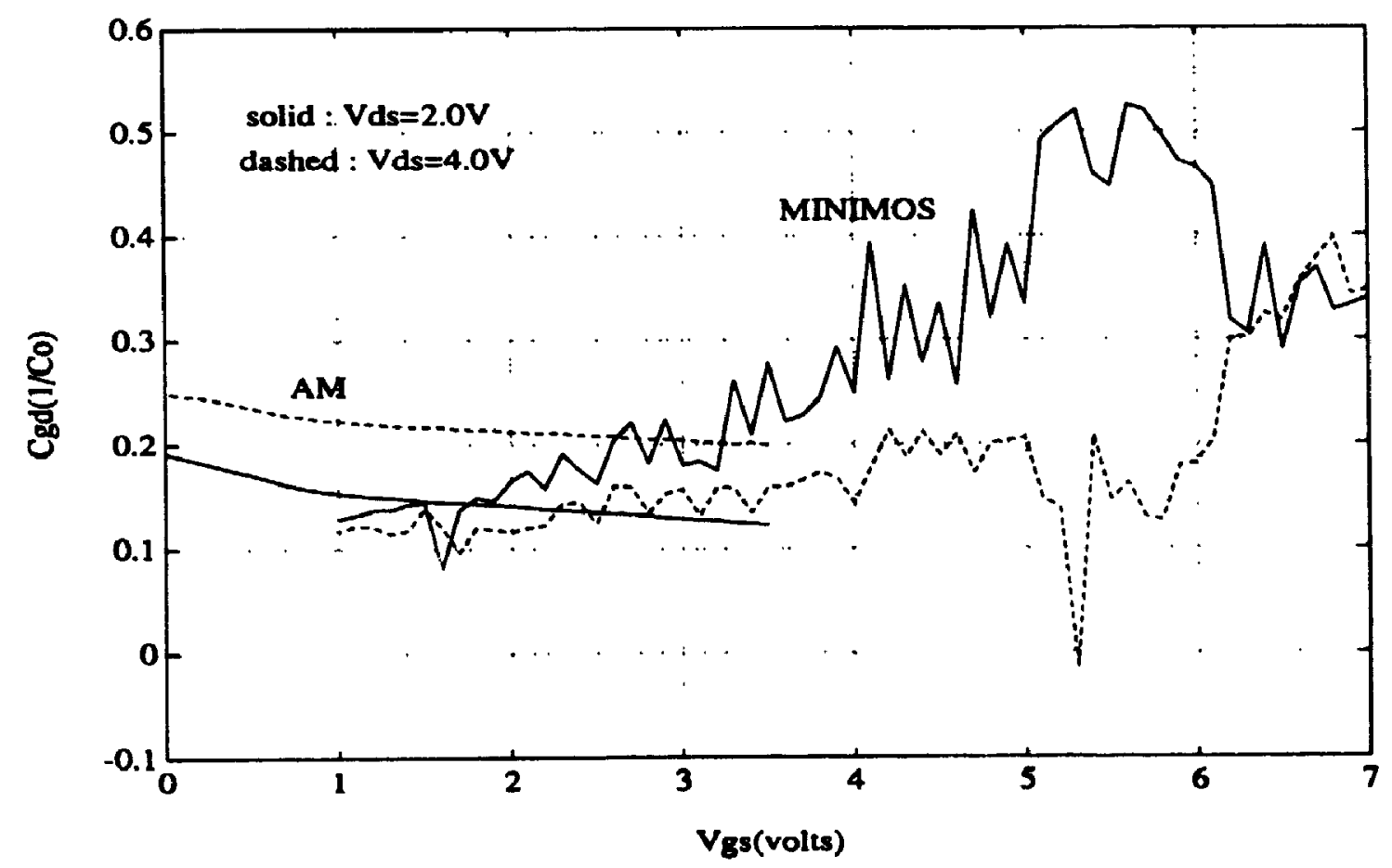

Figure 5.12(b) Normalized $C_{G D}$ obtained from MINIMOS and the AM; $L_{e f f}=1.0 \mu m$. 
occurs well inside the source and drain, as shown by MINIMOS, whereas the AM approximates them to correspond to the potential at the edges of the channel. With these differences in mind, it can be observed that the $\psi_{z}$-distribution of the AM is in reasonably good agreement with that of MINIMOS in the subthreshold region (with $V_{G S}=0.35 \mathrm{~V}$ ), where the differences in the area from the two distributions appear to be equal between the source and drain, and very small. However, the difference between the two models becomes much more apparent when the $\psi_{\mathbf{s}}$-distributions of the saturation region are compared (with $V_{G S}=2.05 \mathrm{~V}$ and $3.05 \mathrm{~V}$ ). In this region of operation, the distributions from MINIMOS begin to rise above those from the AM, immediately adjacent to the source. This difference can be understood from the fact that MINIMOS makes a proper 2D analysis of $\psi_{s}(y)$ throughout the channel, whereas the distributions of $\psi_{s}(y)$ in the source and drain space charge regions obtained from the CJM, completely neglect the influence from the gate which, as seen in the figure, would tend to raise the surface potential in the channel much higher.

Another reason for the differences in the distributions is the change in the mobility $\mu(y)$ with channel length reduction, which is represented by MINIMOS, but which is not taken into account by the analytical model.

Perhaps the most important feature which is correctly predicted by the AM, in agreement with actual measurements on devices[21], is a drain voltage dependence of $C_{G D}$, as discussed at the end of the previous chapter. From Figures 5.12(a) and (b) it is shown that the value of $C_{G D}$ is higher at larger drain voltages, as is to be expected, since the drain space charge region contributes a larger portion of the total distribution $\psi_{s}(y)$, which is changed by modulating $V_{D S}$. It is not clear why this behavior is not shown in the MINIMOS simulations, though if small it is possibly masked by the scatter in the data points. Another possible explanation is inadequate modelling of the mobility $\mu(y)$, velocity saturation and other SCE. 


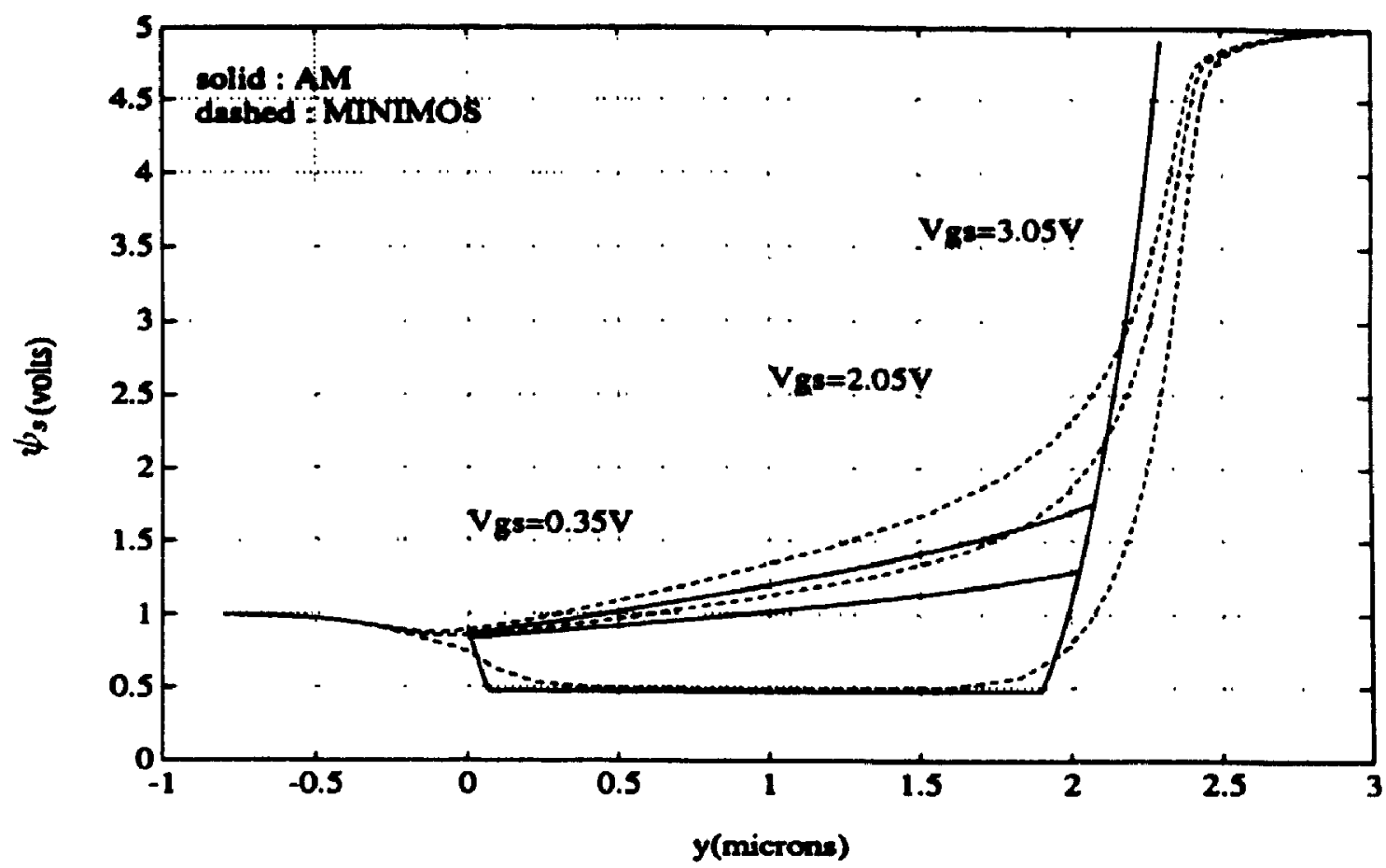

Figure 5.13 $\psi_{s}$-distributions obtained from MINMOS and the AM; $L_{e f f}=2.3 \mu m$.

\subsection{Concluding Remarks}

It has been demonstrated in this chapter that short channel gate capacitances may be obtained from a simple analytical model of the MOSFET; in particular, $C_{G D}$ was evaluated for the MD and the SD, because of the overwhelming importance of the drain to gate interaction in practical circuit applications.

$C_{G D}$ was evaluated using a simple model, which to some degree included the influence of the source and drain space charge regions, in order to demonstrate the possibility of predicting short channel effects in the capacitance characteristics analytically. This could be of value for circuit simulation applications.

In general, by reducing the number of numerical procedures in its implementation, the model may be improved in speed and/or accuracy. For example, the gate charge in each of the three regions, $Q_{G}(s), Q_{G}(c h)$ and $Q_{G}(d)$, was evaluated 


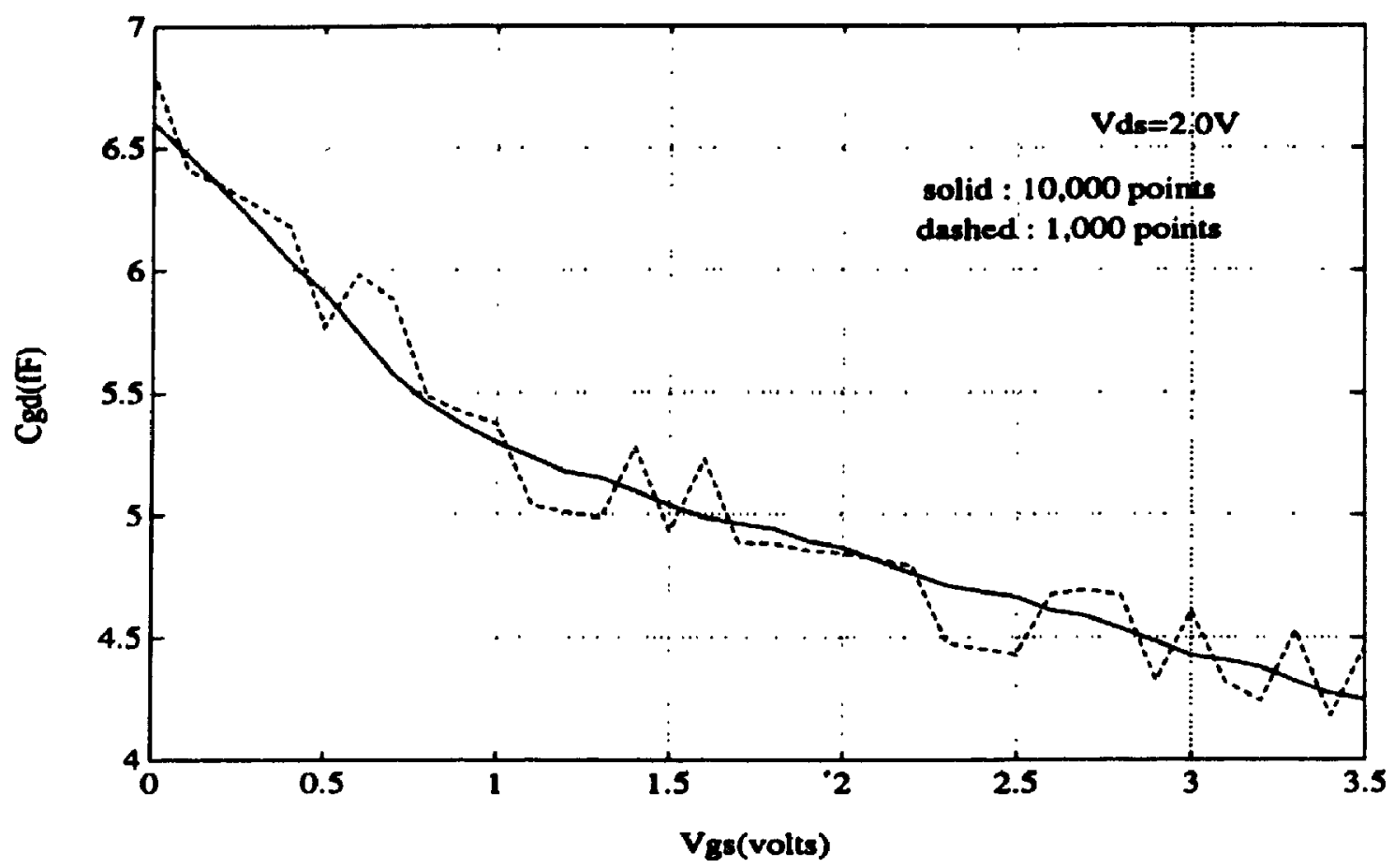

Figure 5.14 $C_{G D} \sim V_{G S} ; L_{e f f}=1.0 \mu m$.

by numerically integrating the quantity $V_{G B}{ }^{\prime}-\psi_{s}(y)$ over the appropriate channel distance, making it necessary to use a very large number of points $\left(10^{4}\right)$ in order to achieve satisfactory smoothness and accuracy of the results. Even so, significant scatter in the calculated capacitance values remains. The penalty paid in the accuracy by reducing the number of points by an order of magnitude is demonstrated in Figure 5.14, showing $C_{G D}$ for the SD at $V_{G S}=2.0 \mathrm{~V}$. Such errors due to numerical integrations would be avoided by integrating the expressions for $Q_{G}$ analytically, thereby ensuring both smooth results and a reduction in computing time, making this method more suitable for practical purposes.

Similarly, the use of the cylindrical step junction model to obtain the distributions $\psi_{s}(y)$ of the source and drain space charge regions may have been further simplified by the use of a quadratic approximation, thus avoiding time-consuming numerical iterations, since the positions $y_{2}$ would then be calculated explicitly. 
The ability to predict accurate short channel capacitance characteristics using a practical analytical model, will depend largely of the degree of sophistication needed in order to adequately represent the true 2D nature of the source and drain space charge regions, including the influence of the gate potential, without incurring a large penalty in computing time. Other effects such as electric field dependence of mobility and velocity saturation, which have been shown to exert considerable influence on the behavior of short channel devices[14,16,19], would need to be represented in a realistic model. 


\section{Chapter 6 \\ Conclusions}

\subsection{Summary}

The objectives of the research presented in this thesis were: 1) to investigate the feasibility of using MINIMOS, a 2D numerical MOSFET simulator, in the study of charge storage and capacitance properties of short channel transistors; 2) to study and explain the resulting characteristics by distinguishing between the features of apparent behavior, introduced by limitations of the simulator, and true physical behavior; 3 ) to investigate the possibility of predicting short channel gate capacitances using an analytical model, which could be feasible for practical circuit simulation applications.

The various physical properties provided as outputs from MINIMOS were studjed to examine the feasibility of using them as the basis of investigation of charge storage and the associated gate capacitances of the MOSFET. It was determined that the gate charge could be calculated with similar accuracy in terms of either the surface potential $\psi_{s}(y)$ or the transverse field $\mathcal{E}_{o x}(y)$ in the oxide; the surface potential was chosen as the most appropriate output quantity for this purpose on grounds of simplicity and convenience.

Discontinuities and numerical scatter in the data from MINIMOS and in the resulting capacitance characteristics, were found to be a serious problem throughout the work. It was found possible to reduce these effects by using the option GRIDFREEZE, in which the simulation grid is held fixed over the whole range of simulations, thereby resulting in far smoother anc more intelligible characteristics. It was determined however. that the numerical scatter is an inherent feature of 
MINIMOS, as presently implemented, due to the limited resolution of points in the channel and in the manner in which these points are distributed.

Smoothing the $Q_{G} \sim V_{G S}$ characteristics using polynomials was found useful as a means of estimating the capacitance behavior, particularly for those curves with relatively little scatter, namely, those of the smaller devices and/or operating at low gate voltage bias conditions. For other conditions of operation it was often possible to estimate the capacitances by hand and eye, as in the case of the LD, using long channel theory as a guide to the true behavior.

Despite the difficulties introduced by scatter of data, useful results showing the effects of channel length reduction of the gate capacitances were obtained. The main features of the characteristics due to short channel effects were determined to be: 1) a shift of the curves to the left, as channel length is reduced; 2) a rounding of $f$ of the capacitance characteristics with operating boundaries less distinguishable in smaller devices; 3) a significant increase in the proportional effect of the inner fringing capacitances $C_{G D}(i f)$ and $C_{G S}($ if $)$ in small devices, which must be represented in realistic and practically useful short channel capacitance models.

It was also shown for the first time that the MOS capacitance $C_{G B}$ is clearly dependent on $V_{D S}$ in the subthreshold region, this being due to the modulation of the drain controlled space charge region of the channel, and constituting a significant proportion of the total channel length in short devices.

A simple analytical model of the MOSFET was investigated in order to demonstrate the possibility of representing capacitance properties of short channel devices in a manner consistent with application in a circuit simulation environment. In particular, the capacitance $C_{G D}$ was evaluated analytically by approximating the change in the surface potential distribution $\Delta \psi_{s}(y)$, caused by modulation of the drain space charge region. In order not to complicate the demonstration beyond the intended scope, the surface potential distribution was approximated using an explicit semi-empirical saturation boundary condition, which permitted the application of the model in the subthreshold and saturation regions of operation. It was 
shown that, though simple, the model was capable of predicting the main properties of the capacitance $C_{G D}$ in these regions, with reasonably good agreement with the results from MINIMOS simulations.

Aside from expanding its range of operation to include the triode region, it was concluded that such a mudel could be improved in both speed and accuracy by evaluating the gate charge analytically and eliminating numerical integration. The degree of sophistication of such a model, to adequately represent 2D effects, would depend on the specific demands of its intended application.

\subsection{Suggestions for Further Studies}

It has been demonstrated that MINIMOS is capable of predicting short channel capacitances and is basically suitable for the study of charge storage and dynamic properties of the MOSFET. Its inadequacies due to uncertainties caused by numerical scatter, have been investigated and have been identified to originate from the simulation grid. On the evidence of other work[14] it is believed that, if modified to allow specification of the grid to be under the control of the user, the scatter of output data could be reduced to negligible order : MINIMOS would then be a powerful tool for the investigation of capacitance properties of short channel MOSFETs. Such an undertaking would require major modifications of the internal code of MINIMOS and would in itself constitute a significant project.

The possibilities of analytical modelling of short channel capacitance properties should be pursued further. The present work has indicated the feasibility of such an apprrach. For its ultimate success, however, an effective way of including the influence of the gate potential on the surface potential distribution in the drain space charge region, with an acceptably low level of analytical complexity, is essential. 


\section{References}

[1] Y. A. El-Mansy, and A. R. Boothroyd, "A New A pproach to the Theory and Modelling of Insulated-Gate Field-Effect Transistors", IEEE Transactions on Electron Devices, Vol. ED-24, No. 3, pp. 241-253, 1977.

[2] Y. A. El-Mansy, and A. R. Boothroyd, "A simple two-dimensional model for IGFET operation in the saturation region", IEEE Transactions on Electron Devices, Vol. ED- 24, No. 3, pp. 254-262, 1977.

[3] J. R. Brews, "A Charge-Sheet Model of the MOSFET", Solid-State Electronics, Vol. 21, pp. 345-355, 1978.

[4] Y.P. Tsividis, Operation and Modelling of the MOS Transistor, McGraw-Hill, 1987.

[5] J. R. Brews, "Physics of the MOS Transistor", Chapter 1 in Silicon Integrated Circuits, Part A, D. Kaling (editor), Applied Solid-State Series, Academic Press, N. Y., 1981.

[6] J.A. Robinson, Y.A. El-Mansy, and A.R. Boothroyd, "A General Four-Terminal Charging-Current Model for the Insulated-Gate Field-Effect Transistor-I", Solid State Electronics, Vol. 23, pp. 405-410, Pergamon Press Ltd., 1980.

[7] C. Turchetti, G. Masetti and Y. Tsividis, "On the Small-Signal Behavior of the MOS Transistor in Quasi-Static Operation", Solid-State Electronics, Vol. 26, pp. 941-949, 1983.

[8] J.E. Meyer, "MOS Models and Circuit Simulation", RCA Review, Vol. 32, pp. 42-63, RCA Laboratories, March 1971.

[9] D.E. Ward and R.W. Dutton, "A Charge-Oriented Model for MOS Transistor Capacitances", IEEE Journal of Solid-State Circuits, Vol. SC-13, No. 5, pp. 703-708, Oct. 1978.

[10] Dr. A. R. Boothroyd, "private communication".

[11] P. Yang, B.D. Epler and P.K. Chatterjee, "An Investigation of the Charge Con servation Problem for MOSFET Circuit Simulation", IEEE Journal of Solid. State Circuits, Vol. SC-18, No. 1, pp. 128-138, Feb. 1983.

[12] D.E. Ward, "Charge-Based Modelling of Capacitance in MOS Transistors", Technical Report, June 1981. 
[13] P. Prioretti, C.Turchetti, G. Masetti, E. Profumo and M. Vanzi, "A Meyer-Like Model for the MOST Satisfying Gate Charge Conservation", pp. 84-86, 1985.

[14] S. S. S. Chung, "A Charge-Based Capacitance Model of Short-Channel MOSFETs", IEEE iranaactions on Computer-Aided Design, Vol. 8, No. 1, Jan. 1989.

[15] S.Y. Oh, D.E. Ward and R.W. Dutton, "Transient Analysis of MOS Transistors", IEEE Transactions on Electron Devices, Vol. ED-27, No.8, pp.1571-1578, Aug. 1980.

[16] H. Iwai, M.R. Pinto, C.S. Rafferty, J.E. Oristian and R.W. Dutton, "Analysis of Velocity Saturation and Other Effects on Short-Channel MOS Transistor Capacitances", IEEE Transactions on Computer-Aided Design, Vol. CAD-6, No. 2, pp. 173-184, March 1987

[17] H. Iwai, J. Oristian, J. Walker and R. Dutton, "Small Geometry MOS Transisistor Measurements and Observed Short and Narrow Channel Effects", Digest of Technical Papers, Symposium on VLSI Technology, San Diego CA, pp. 7879,Sept. 1985.

[18] Y. Ohkura, T. Toyabe, H. Masuda, "Analysis of MOSFET Capacitances and Their Behaviour at Short-Channel Lengths Using and AC Device Simulator", IEEE Transactions on Computer-Aided Design, Vol. CAD-6, No. 2, March 1987.

[19] Y.T. Yeow, "Measurement and Numerical Modelling of Short-Channel MOSFET Gate Capacitances", IEEE Transections on Electron Devices, Vol. ED35, No. 12, pp. 2510-2520, Dec. 1987.

[20] H. Iwai, M.R. Pinto, C.S. Rafferty, J.E. Oristian and R.W. Dutton, "Velocity Saturation Effect on Short-Channel MOS Transistor Capacitance", IEEE Elec tron Device Letters, Vol. EDL-6, No. 3, pp. 120-122, March 1985.

[21] B.J. Sheu and P.K. Ko, "An Analytical Model for Intrinsic Capacitances of Short-Channel MOSFET's", IEDM, pp. 300-302, 1984.

[22] M. R. Pinto, C. R. Rafferty and R. W. Dutton, "PISCES-II: Poisson and Continuity Equation Solver", Stanford Electronics Lab., Stanford University, Sept. 1984.

[23] S.M Sze, Physics of Semiconductor Devices, 2nd Edition, Wiley-Interscience, 1981.

[24] A.S. Grove, Physics and Technology of Semiconductor Devices, John Wiley \& 
Sons Inc., 1967.

[25] L. D. Yau, "A Simple Theory to Predict the Threshold Voltage of Short-Channel IGFET's", Solid State Electronics, Vol. 17, pp. 1059, 1974.

[26] S. Selberherr, A. Schutz and H.W. Potzl, "MINIMOS-A Two Dimensional MOS Transistor Analyzer", IEEE Transactions on Electron Devices, Vol. ED- 27, No. 8, pp. 1540-1549, Aug. 1980. 

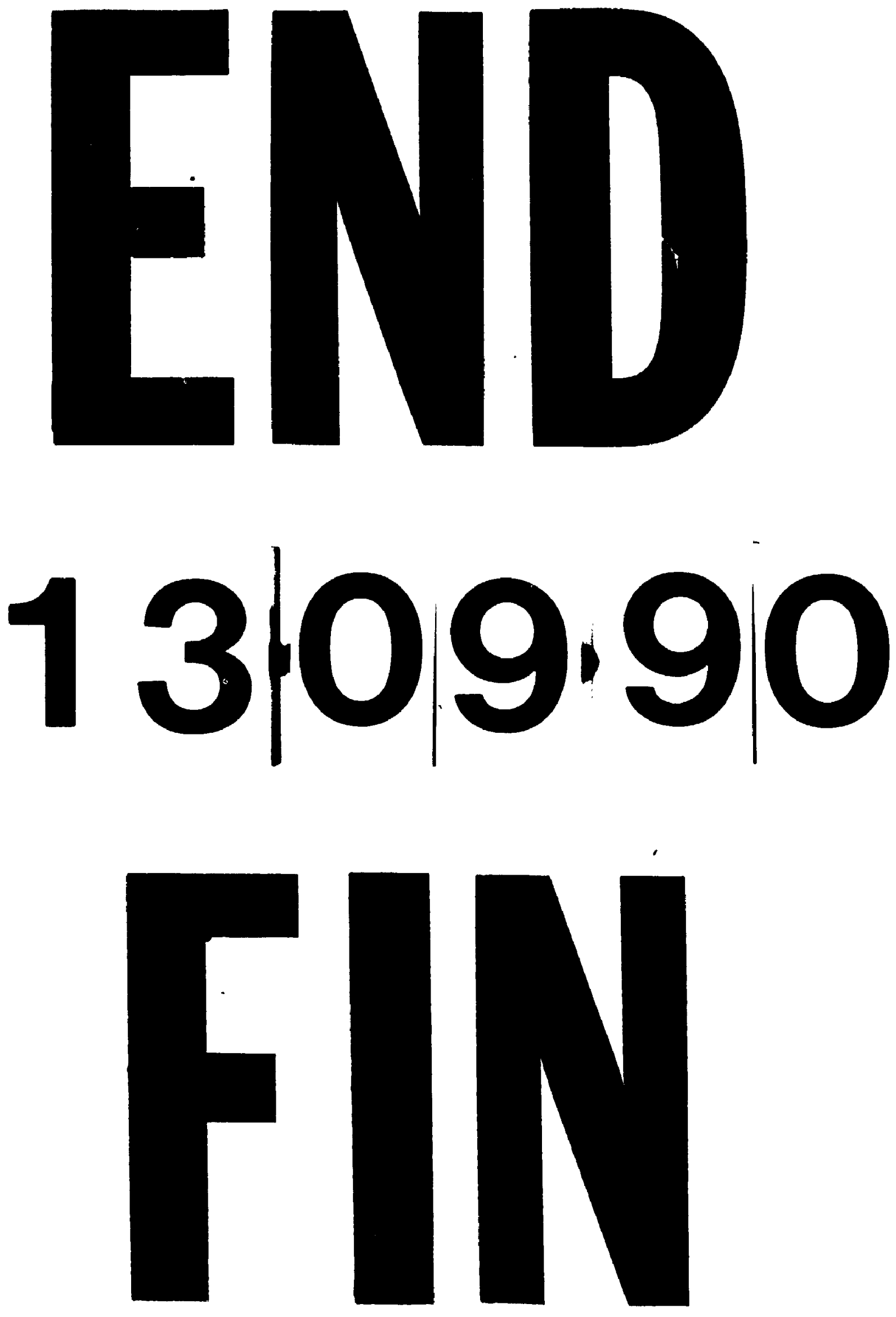